WSRC-TR-2000-00011

Revision 0

\title{
TOTAL INVENTORY OF SELECTED RADIONUCLIDES IN OLD SOLVENT TANKS S1 THROUGH S22 (U)
}

\author{
John D. Leyba \\ Unclassified \\ Does Not Contain Unclassified Controlled Nuclear Information (UCNI)
}

January 21, 2000

Westinghouse Savannah River Company

Savannah River Site

Aiken, SC 29808

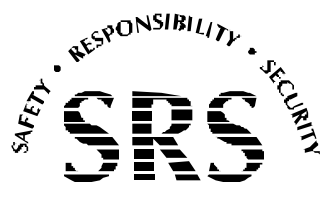

SAVANNAH RIVER SITE 
WSRC-TR-2000-00011

Revision 0

Keywords: Characterization Radioactive Solvent Waste

Classification: U

Authorized Derivative Classifier

TOTAL INVENTORY OF SELECTED RADIONUCLIDES IN OLD SOLVENT

TANKS S1 THROUGH S22 (U)

\section{J. D. Leyba}

Publication Date: January 21, 2000

J. D. Leyba, Author

$\overline{\text { Date }}$

V. R. Casella, Technical Reviewer

Date

P. E. Filpus-Luyckx, ADS L4 Manager

Date

Westinghouse Savannah River Company

Savannah River Site

Aiken, SC 29808

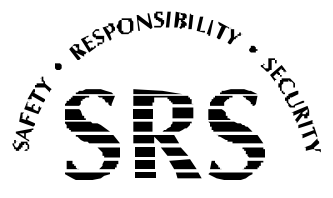

SAVANNAH RIVER SITE 
This document was prepared in conjunction with work accomplished under Contract No. DE-AC09-96SR18500 with the U.S. Department of Energy.

\section{DISCLAIMER}

This report was prepared as an account of work sponsored by an agency of the United States Government. Neither the United States Government nor any agency thereof, nor any of their employees, makes any warranty, express or implied, or assumes any legal liability or responsibility for the accuracy, completeness, or usefulness of any information, apparatus, product or process disclosed, or represents that its use would not infringe privately owned rights. Reference herein to any specific commercial product, process or service by trade name, trademark, manufacturer, or otherwise does not necessarily constitute or imply its endorsement, recommendation, or favoring by the United States Government or any agency thereof. The views and opinions of authors expressed herein do not necessarily state or reflect those of the United States Government or any agency thereof.

This report has been reproduced directly from the best available copy.

Available for sale to the public, in paper, from: U.S. Department of Commerce, National Technical Information Service, 5285 Port Royal Road, Springfield, VA 22161, phone: (800) 553-6847, fax: (703) 605-6900, email: orders@ ntis.fedworld.gov online ordering: http://www.ntis.gov/ordering.htm

Available electronically at http://www.doe.gov/bridge

Available for a processing fee to U.S. Department of Energy and its contractors, in paper, from: U.S. Department of Energy, Office of Scientific and Technical Information, P.O. Box 62, Oak Ridge, TN 37831-0062, phone: (865 ) 576-8401, fax: (865) 576-5728, email: reports@adonis.osti.gov 


\begin{abstract}
The total inventory of fourteen radionuclides, three metals, and volatile organic compounds (VOCs) has been calculated for the twenty-two Old Solvent Tanks (OSTs). The inventory calculations are based upon extensive characterization data of the multiple liquid and sludge samples taken from the OSTs. In addition, the total inventory of sixteen actinides (including error) has been calculated. The actinide inventory will be useful for criticality safety considerations.
\end{abstract}


WSRC-TR-2000-00011

Revision 0

January 21, 2000

Page v

This page intentionally left blank. 


\section{TABLE OF CONTENTS}

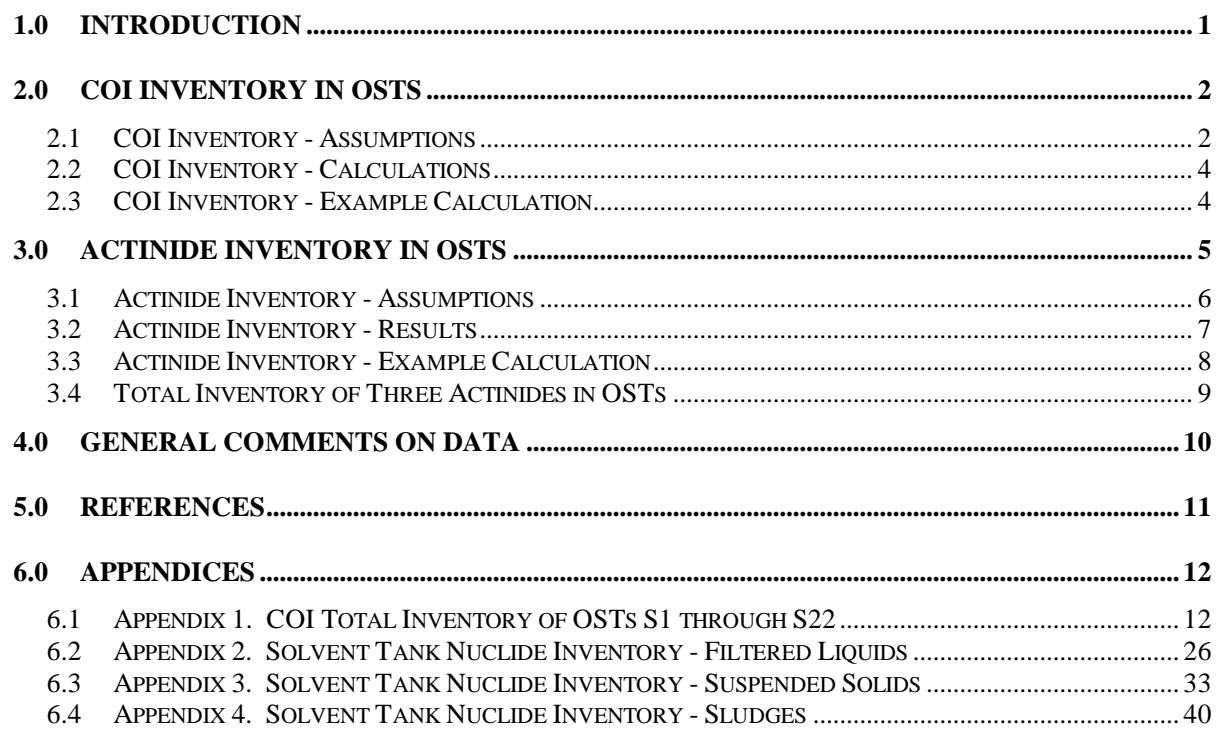

\section{LIST OF TABLES}

Table 1. Total Estimated U-235, U-238, and Pu-239 Content of Old Solvent Tanks S1 through S22

Table 2. Comparison of Activity Levels in Suspended Solids and Sludge...................10 
WSRC-TR-99-00414

Revision 0

November 1, 1999

Page vii

\section{LIST OF ACRONYMS USED}

ADS Analytical Development Section

COI Contaminant of Interest

ORWBG Old Radioactive Waste Burial Ground

OSTs Old Solvent Tanks

PUREX Plutonium-Uranium Extraction

SRS-ERD Savannah River Site-Environmental Restoration Division

SRTC Savannah River Technology Center

VOCs Volatile Organic Compounds 


\title{
TOTAL INVENTORY OF SELECTED RADIONUCLIDES IN OLD SOLVENT TANKS S1 THROUGH S22 (U)
}

\author{
J. D. Leyba \\ Westinghouse Savannah River Company \\ Savannah River Site \\ Aiken, SC 29808
}

\subsection{INTRODUCTION}

The Old Radioactive Waste Burial Ground (ORWBG, 643-E) contains twenty-two buried old solvent tanks. These tanks, consisting of cylindrical, carbon-steel, single-wall vessels were used to store spent Plutonium-Uranium Extraction (PUREX) process solvent.

Reference 1 provides a detailed description of the tanks and their associated history. The Analytical Development Section (ADS) of the Savannah River Technology Center (SRTC) developed a Sampling and Analysis Plan for the characterization of the material contained in the twenty-two tanks [2]. The purpose of this characterization was to obtain a sufficient database so that a viable path forward could be established for the closure of the tanks. Reference 3 contains the analytical results of the OST characterization study.

Due to various reasons, not all of the phases present in all of the tanks were sampled. As discussed in Reference 3, liquid samples were successfully obtained from fifteen out of twenty-two tanks. Of the remaining seven tanks, three were not sampled because of the presence of only very minimal amounts of liquid and four tanks contained no liquid phase. For the sludges, nine out of twenty-two tanks were successfully sampled while only very limited sludge samples were obtained from three other tanks. The Sampling and Analysis Plan did not require sampling in nine of the ten remaining tanks because they contained less than 50 gallons each of solid phase. Unsuccessful attempts were made; however, to sample the sludge in some of these remaining tanks. Thus, only one tank (Tank S5), which was required to be sampled by the Sampling and Analysis Plan, was not sampled.

The Savannah River Site-Environmental Restoration Division (SRS-ERD) has subsequently requested an estimate of the total inventory of eighteen Contaminants of Interest (COIs) for all twenty-two tanks. Included in the COI list are H-3, C-14, I-129, Cs-137, Pu-238, Pu-239, Pu-241, Sr-90, U-235, U-238, Co-60, Tc-99, Np-237, Cm-244, $\mathrm{Cd}, \mathrm{Pb}, \mathrm{Hg}$, and total VOCs. Also, an estimate of the total inventory of sixteen actinides, including seven COIs, was calculated. The actinide list includes U-233, U-234, U-235, 
U-236, U-238, Np-237, Pu-238, Pu-239, Pu-240, Pu-241, Pu-242, Am-241, Am-243, Cm244, Cm-245, and Cm-246. The sixteen actinides are important for criticality considerations.

\subsection{COI Inventory in OSTs}

In order to accurately estimate the total inventory of the eighteen COIs contained in the OSTs, one must systematically categorize the contents of each tank. Based upon previous video and physical surveillances of the tanks, an estimated sludge mass and liquid volume was determined for each tank [4][5][6]. Once liquid samples were obtained and submitted to ADS for characterization, they were filtered to obtain separate fractions of liquid and suspended solids material. Each fraction was subsequently analyzed for the various COIs. Sludge samples were also independently analyzed by ADS. Analytical data was thus obtained, by phase, for many of the OSTs.

A methodology was therefore developed to accurately estimate the inventory of those tank phases that were not sampled. This methodology uses as its starting point the latest analytical data of the various phases present (e.g. aqueous liquid, suspended solids, and sludge) in each tank. For a specific phase (filtered liquid, filtered solid, or sludge), the methodology assumes that the concentration of a given COI in those tanks that were not sampled is equal to the average concentration of the COI in the tanks that were sampled and analyzed. Three general cases were identified and are delineated below:

1) COI detected in more than three samples of a given phase - the analytical data were averaged and the COI concentration in those tanks which were not sampled was assumed to be equal to this calculated average COI concentration.

2) COI detected in less than or equal to three samples of a given phase - the COI concentration in those tanks which were not sampled was assumed to be equal to the higher of the average detected COI concentration or the average detection limit value.

3) COI not detected in any samples of a given phase - the COI concentration in those tanks not sampled was assumed to be equal to the average detection limit value or average upper limit value of those tanks that were sampled.

Once the average COI concentrations were assigned, the total inventories could easily be calculated by taking the product of the activity concentration and the volume or mass of phase present in a given tank.

\subsection{COI Inventory - Assumptions}


The characterization of the material in the OSTs showed that at least two of the tanks (Tanks S11 and S20) contained an organic liquid phase [3]. In addition, Tank S20 contained both organic and aqueous liquid layers. For the purposes of these calculations, it was assumed that Tanks S1-S8, S10, S12-S14, S18, S19, S21 and S22 contained an aqueous liquid phase and no organic liquid phase. Furthermore, it was assumed that Tank S11 contained an organic liquid phase and no aqueous liquid phase. Finally, it was assumed that Tank S20 contained both an aqueous liquid phase and an organic liquid phase. It should be noted that only the total liquid volume in Tank S20 was estimated, thus the fraction that is aqueous and the fraction that is organic were not known. For the sake of conservatism, when performing the inventory calculations, it was assumed that the entire liquid volume was aqueous when performing the aqueous liquid calculations and subsequently it was assumed that the entire liquid volume was organic when performing the organic liquid calculations. It should be noted that several tanks in addition to Tanks S11 and S20 might also contain an organic liquid phase. This is discussed in detail in Reference 1. The assumptions used for the calculations in this present document regarding the aqueous/organic nature of the various liquids in the OSTs are based upon the analytical results (which are documented in Reference 3) of the latest samples obtained.

Two liquid samples were obtained from Tank S19 and both were analyzed. The analytical results indicated that both samples were aqueous; hence, the samples can be treated as duplicates. See Reference 3 for more details regarding this issue. Therefore, when averaging the aqueous liquid phases and associated suspended solids, only sample S19-O (taken at the higher depth) was included in the calculations.

The organic liquids contained in Tanks S11 and S20 were not included when calculating the average concentrations of the various COIs in the filtered aqueous liquids. Similarly, the suspended solids from the organic liquids in Tanks S11 and S20 were not included when calculating the average concentrations of the various COIs in the suspended solids from aqueous liquids. The sludge obtained from Tank S4 was not included when determining the average concentrations of the eighteen COIs due to the fact that it displayed the characteristics of a liquid rather than sludge.

The liquids contained in Tanks S10, S12, and S18 were not sampled. However, the total suspended solids content had to be estimated so that the COI content of the suspended solids could be calculated. The weight percent of total suspended solids for these three tanks was assumed to be equal to the average weight percent of total suspended solids for the aqueous phases of the other tanks. When performing these suspended solids calculations, the density of the filtered liquids in Tanks S10, S12, and S18 was assumed to be equal to $1.0 \mathrm{~g} / \mathrm{cm}^{3}$.

Certain radionuclides were not detected; hence, the average concentration of these nuclides had to be calculated based upon detection limit or upper limit values. These radionuclides are listed below: 
1) The average $\mathrm{H}-3$ activity concentration in sludge is based upon upper limit values.

2) The average Co-60 activity concentration in sludge is based upon detection limit values.

3) The average C-14 activity concentration in the filtered liquids is based upon upper limit values.

4) The average C-14 activity concentration in the suspended solids is based upon upper limit values.

5) The average I-129 activity concentration in the suspended solids is based upon upper limit values.

6) The average Tc-99 activity concentration in the suspended solids is based upon detection limit values.

The C-14, I-129, Sr-90, and Tc-99 average concentrations in the sludge are based upon data obtained from the suspended solids. The implications of this assumption are discussed further in Section 4.0.

Since quantitative metals data were not obtained on the suspended solids, fifty times the average concentrations of $\mathrm{Cd}, \mathrm{Pb}$, and $\mathrm{Hg}$ in the sludge were used to calculate the content of these metals in the suspended solids. This issue is also discussed further in Section 4.0.

The VOC content of both the suspended solids material and sludge was based upon the filtered liquid data and corrected for the expected difference in density between the materials. For these calculations, it was assumed that the VOC average mass per unit volume for the suspended solids and sludge was equal to that of the filtered liquids. It was also assumed that the density of the sludge and suspended solids was $2 \mathrm{~g} / \mathrm{cm}^{3}$. The average density of the filtered liquids was approximately $1 \mathrm{~g} / \mathrm{cm}^{3}$.

\subsection{COI Inventory - Calculations}

The results of the COI inventory calculations are shown in Appendix 1. Results are listed by phase for each tank for the eighteen COIs. Values shown in bold black print are detection limit values. Values shown in bold red are explained in the notes of Appendix 1 and represent calculated upper limit values. Values shown in bold blue represent calculated values (using the methodology explained in Sections 2.0 and 2.1) for those phases that were not analyzed. The total activities for the radionuclides and total mass for the other COIs are shown on pages 13 and 16 respectively of Appendix 1.

\subsection{COI Inventory - Example Calculation}

The calculated H-3 activity in the Tank S1 filtered liquid will be used as an example. 
The average aqueous $\mathrm{H}-3$ concentration was calculated from the analytical results of Tanks S1, S2, S3, S4, S6, S7, S8, S13, S14, S19-O, S20-B, S21, and S22. The average H-3 concentration was determined to be $1.97 \times 10^{-3} \mu \mathrm{Ci} / \mathrm{mL}$. The estimated aqueous liquid volume in Tank S12 was determined to be $1.890 \times 10^{4} \mathrm{~mL}$, thus,

$$
1.97 \times 10^{-3} \mu \mathrm{Ci} / \mathrm{mL} \times 1.890 \times 10^{4} \mathrm{~mL}=3.72 \times 10^{1} \mu \mathrm{Ci} .
$$

\subsection{Actinide Inventory in OSTs}

The existing analytical data (for those tank phases that were analyzed) were used as a starting point for the actinide inventory calculations. For each specific phase (filtered liquid, filtered solid, or sludge), the calculation methodology assumes that the concentration of a given actinide in those tanks that were not sampled is equal to the average concentration of the actinide in the tanks that were sampled. As with the eighteen COIs, three general cases for the actinide calculations were identified and are listed below:

1) Radionuclide detected in more than three samples of a given phase - the analytical data were averaged and the radionuclide concentration in those tanks that were not sampled was assumed to be equal to this calculated average radionuclide concentration.

2) Radionuclide detected in less than or equal to three samples of a given phase - the radionuclide concentration in those tanks which were not sampled was assumed to be equal to the higher of either the average detected radionuclide concentration or the average detection limit value.

3) Radionuclide not detected in any samples of a given phase - the radionuclide concentration in those tanks not sampled was assumed to be equal to the average detection limit value or average upper limit value of those tanks that were sampled.

The error associated with the calculated average activity for each radionuclide was assigned as twice the standard deviation of the respective average. When detection limits were averaged, no errors were assigned since these numbers represent $\sim 95 \%$ confidence limits.

Since the actinide inventory will be used for criticality safety calculations, conservatism was built into the calculation methodology. Firstly, for a radionuclide known or suspected to be in the OSTs but not detected in a sample, the activity was assumed to be equal to the detection limit or upper limit for that radionuclide for the measurement conditions used. Secondly, for those nuclides that were positively identified, the 2-sigma measurement error was added to the activity before performing the total activity and mass calculations. For those tanks in which a radionuclide was not measured for a given phase and an average radionuclide concentration was assigned, the calculated error (see previous paragraph) was 
added to the calculated radionuclide activity concentration prior to performing the total activity and mass calculations.

\subsection{Actinide Inventory - Assumptions}

The assumptions for the actinide inventory calculations are similar to those used for the eighteen COIs described in Section 2.1. The characterization of the material in the OSTs showed that at least two of the tanks (Tanks S11 and S20) contained an organic liquid phase [3]. In addition, Tank S20 contained both organic and aqueous liquid layers. For the purposes of these calculations, it was assumed that Tanks S1-S8, S10, S12-S14, S18, S19, S21 and S22 contained an aqueous liquid phase and no organic liquid phase. Furthermore, it was assumed that Tank S11 contained an organic liquid phase and no aqueous liquid phase. Finally, it was assumed that Tank S20 contained both an aqueous liquid phase and an organic liquid phase. It should be noted that only the total liquid volume in Tank S20 was estimated, thus the fraction that is aqueous and the fraction that is organic were not known. For the sake of conservatism, when performing the inventory calculations, it was assumed that the entire liquid volume was aqueous when performing the aqueous liquid calculations and subsequently it was assumed that the entire liquid volume was organic when performing the organic liquid calculations. It should be noted that several tanks in addition to Tanks S11 and S20 might also contain an organic liquid phase. This is discussed in detail in Reference 1 . The assumptions used for the calculations in this present document regarding the aqueous/organic nature of the various liquids in the OSTs are based upon the analytical results (which are documented in Reference 3) of the latest samples obtained.

Two liquid samples were obtained from Tank S19 and both were analyzed. The analytical results indicated that both samples were aqueous; hence, the samples can be treated as duplicates. See Reference 3 for more details regarding this issue. Therefore, when averaging the aqueous liquid phases and associated suspended solids, only sample S19-O (taken at the higher depth) was included in the calculations.

The organic liquids contained in Tanks S11 and S20 were not included when calculating the average concentrations of the various actinides in the filtered aqueous liquids. Similarly, the suspended solids from the organic liquids in Tanks S11 and S20 were not included when calculating the average concentrations of the various actinides in the suspended solids from aqueous liquids. The sludge obtained from Tank S4 was not included when determining the average concentrations of the sixteen actinides due to the fact that it displayed the characteristics of a liquid rather than sludge.

The liquids contained in Tanks S10, S12, and S18 were not sampled. However, the total suspended solids content had to be estimated so that the actinide content of the suspended solids could be calculated. The weight percent of total suspended solids for these three 
tanks was assumed to be equal to the average weight percent of total suspended solids for the aqueous phases of the other tanks. When performing these suspended solids

calculations, the density of the filtered liquids in Tanks S10, S12, and S18 was assumed to be equal to $1.0 \mathrm{~g} / \mathrm{cm}^{3}$.

Certain radionuclides were not detected; hence, the average concentration of these nuclides had to be calculated based upon detection limit or upper limit values. These radionuclides are listed below:

1) The average $\mathrm{Pu}-242$ activity concentration in sludge is based upon detection limit values.

2) The average Am-243 activity concentration in sludge is based upon detection limit values and one upper limit value, even though this nuclide was detected in three sludges. However, based upon rule 2 of Section 3.0, the higher average detection limit/upper limit value was used.

3) The average $\mathrm{Cm}-245$ activity concentration in sludge is based upon detection limit values even though this nuclide was detected in one sludge. However, based upon rule 2 of Section 3.0, the higher average detection limit value was used.

4) The average $\mathrm{Cm}-246$ activity concentration in the sludge is based upon detection limit values.

5) The average $\mathrm{Pu}-242$ activity concentration in the filtered liquids is based upon detection limit values.

6) The average $\mathrm{Cm}-245$ activity concentration in the filtered liquids is based upon detection limit values.

7) The average $\mathrm{Cm}-246$ activity concentration in the filtered liquids is based upon detection limit values.

8) The average Cm-245 activity concentration in the suspended solids is based upon detection limit values even though this nuclide was detected in one suspended solids sample. However, based upon rule 2 of Section 3.0, the higher average detection limit value was used.

9) The average Cm-246 activity concentration in the suspended solids is based upon detection limit values even though this nuclide was detected in three suspended solids samples. However, based upon rule 2 of Section 3.0, the higher average detection limit value was used.

\subsection{Actinide Inventory - Results}

The results of the actinide inventory calculations are shown in Appendices 2 through 4. Results are listed by phase for each tank for the sixteen actinides. Values shown in bold black print are detection limit values. Values shown in bold blue represent calculated values (using the methodology explained in Sections 3.0 and 3.1) for those phases that were not analyzed. 


\subsection{Actinide Inventory - Example Calculation}

The calculated U-234 activity and error in the Tank S1 sludge will be used as an example. The average U-234 concentration was calculated from the analytical results of Tanks S7, $\mathrm{S} 19$ and S22. The average U-234 concentration was determined to be $5.02 \times 10^{4} \mathrm{pCi} / \mathrm{g}$ (see page 40 of Appendix 4). The error was calculated to be $1.43 \times 10^{5} \mathrm{pCi} / \mathrm{g}$. Since

U-234 was found in only three of the sludge samples, the average U-234 detection limit in the other analyzed sludge samples was also calculated (see Section 3.0). Thus, the average U-234 detection limit calculated from Tanks S2, S8, S16, S20, and S21 was determined to be $1.32 \times 10^{4} \mathrm{pCi} / \mathrm{g}$. Therefore, for the purposes of these calculations, the higher calculated activity concentration $\left(5.02 \times 10^{4} \mathrm{pCi} / \mathrm{g}\right)$ was used.

Continuing on with the example calculation using U-234 in the sludge from Tank S1 we get:

$\mathrm{U}-234$ activity in sludge $=5.02 \times 10^{4} \pm 1.43 \times 10^{5} \mathrm{pCi} / \mathrm{g}$

Specific Activity of $\mathrm{U}-234=6.24 \times 10^{-3} \mathrm{Ci} / \mathrm{g}$

Mass of sludge in the tank $=34068.71$ grams

Total U-234 in the tank including error $=\left(5.02 \times 10^{4}+1.43 \times 10^{5}\right) \times 34068.71=6.582 \times 10^{9} \mathrm{pCi}$

Total U-234 in the tank including error $=6.582 \times 10^{9} \mathrm{pCi} \times \frac{1 \mathrm{Ci}}{1 \times 10^{12} \mathrm{pCi}} \approx 6.58 \times 10^{-3} \mathrm{Ci}$

$\mathrm{U}-234$ activity concentration including error $=\frac{6.582 \times 10^{-3} \mathrm{Ci}}{34068.71 \text { grams }}=1.93 \times 10^{-7} \mathrm{Ci} / \mathrm{g}$

Mass of U-234 in the tank including error $=\frac{6.582 \times 10^{-3} \mathrm{Ci}}{6.24 \times 10^{-3} \mathrm{Ci} / \mathrm{g}}=1.055 \times 10^{0} \approx 1.06 \times 10^{0} \mathrm{~g}$ 
Mass of U-234 per gram of waste $=\frac{1.055 \times 10^{0} \text { grams }}{34068.71 \text { grams }}=3.097 \times 10^{-5} \approx 3.10 \times 10^{-5} \mathrm{~g} / \mathrm{g}($ waste $)$

\subsection{Total Inventory of Three Actinides in OSTs}

Table 1 summarizes the results of the U-235, U-238, and Pu-239 mass calculations for the twenty-two OSTs. For each of these nuclides in a given tank, the contributions from the filtered liquid phase(s), suspended solids phase(s), and sludge phase have been summed to obtain the total.

Table 1. Total Estimated U-235, U-238, and Pu-239 Content of Old Solvent Tanks S1 through S22

\begin{tabular}{|c|c|c|c|}
\hline Tank \# & $\begin{array}{c}\mathbf{U - 2 3 5} \\
\mathbf{( g )}\end{array}$ & $\begin{array}{c}\mathbf{U - 2 3 8} \\
\mathbf{( g )}\end{array}$ & $\begin{array}{c}\text { Pu-239 } \\
\mathbf{( g )}\end{array}$ \\
\hline S1 & $6.18 \mathrm{E}+01$ & $8.98 \mathrm{E}+03$ & $<8.16 \mathrm{E}-01$ \\
\hline S2 & $8.89 \mathrm{E}+00$ & $1.45 \mathrm{E}+04$ & $<1.25 \mathrm{E}+00$ \\
\hline S3 & $1.07 \mathrm{E}+02$ & $1.59 \mathrm{E}+04$ & $1.30 \mathrm{E}+00$ \\
\hline S4 & $<1.67 \mathrm{E}+02$ & $3.43 \mathrm{E}+04$ & $<1.04 \mathrm{E}+00$ \\
\hline S5 & $1.08 \mathrm{E}+03$ & $1.60 \mathrm{E}+05$ & $1.80 \mathrm{E}+01$ \\
\hline S6 & $7.84 \mathrm{E}+01$ & $1.58 \mathrm{E}+04$ & $<2.51 \mathrm{E}+00$ \\
\hline S7 & $2.33 \mathrm{E}+02$ & $4.88 \mathrm{E}+04$ & $3.11 \mathrm{E}+00$ \\
\hline S8 & $7.60 \mathrm{E}+00$ & $1.16 \mathrm{E}+03$ & $<5.22 \mathrm{E}-01$ \\
\hline S9 & $1.18 \mathrm{E}+02$ & $1.73 \mathrm{E}+04$ & $1.92 \mathrm{E}+00$ \\
\hline S10 & $2.86 \mathrm{E}+02$ & $4.25 \mathrm{E}+04$ & $4.76 \mathrm{E}+00$ \\
\hline S11 & $1.15 \mathrm{E}+02$ & $<1.69 \mathrm{E}+04$ & $1.92 \mathrm{E}+00$ \\
\hline S12 & $4.80 \mathrm{E}+01$ & $7.30 \mathrm{E}+03$ & $8.30 \mathrm{E}-01$ \\
\hline S13 & $4.52 \mathrm{E}+02$ & $6.74 \mathrm{E}+04$ & $<7.18 \mathrm{E}+00$ \\
\hline S14 & $1.11 \mathrm{E}+03$ & $1.75 \mathrm{E}+05$ & $1.78 \mathrm{E}+01$ \\
\hline S15 & $1.31 \mathrm{E}+03$ & $1.93 \mathrm{E}+05$ & $2.14 \mathrm{E}+01$ \\
\hline S16 & $1.02 \mathrm{E}+03$ & $1.87 \mathrm{E}+05$ & $2.86 \mathrm{E}+01$ \\
\hline S17 & $6.33 \mathrm{E}+01$ & $9.31 \mathrm{E}+03$ & $1.03 \mathrm{E}+00$ \\
\hline S18 & $2.09 \mathrm{E}+01$ & $3.31 \mathrm{E}+03$ & $3.88 \mathrm{E}-01$ \\
\hline S19 & $3.68 \mathrm{E}+02^{\mathrm{a}}$ & $8.77 \mathrm{E}+04^{\mathrm{a}}$ & $4.24 \mathrm{E}+00^{\mathrm{a}}$ \\
\hline S20 & $<2.22 \mathrm{E}+02$ & $<5.33 \mathrm{E}+04$ & $<3.50 \mathrm{E}+00$ \\
\hline S21 & $2.75 \mathrm{E}+01$ & $8.78 \mathrm{E}+03$ & $9.83 \mathrm{E}-01$ \\
\hline S22 & $5.07 \mathrm{E}+01$ & $<1.59 \mathrm{E}+04$ & $<1.62 \mathrm{E}+00$ \\
\hline
\end{tabular}

a- This value was calculated using the results from sample 19-O. 


\subsection{GENERAL COMMENTS ON DATA}

The various samples from the OSTs presented ADS with a unique analytical challenge. It is extremely difficult to determine the quantities of trace radionuclides in the presence of gross amounts of other radionuclides. This is especially pertinent to constituents such as C-14, I-129, Ni-59, and Ni-63. For example, the gross beta activity level in the Tanks S1 filtered liquid was determined to be $1.82 \mu \mathrm{Ci} / \mathrm{mL}$ [3]. The $\mathrm{C}-14$ upper limit value in this liquid was determined to be $3.50 \times 10^{-4} \mu \mathrm{Ci} / \mathrm{mL}$, which represents less than $0.02 \%$ of the total activity. It should also be emphasized that the finite sample size available for analysis hindered the achievement of lower detection limit numbers.

Carbon-14 was not actually detected in any of the OST samples that were analyzed for this constituent. In all cases but one, the C-14 analytical data had to be qualified as upper limit values due to the presence of alpha activity in the separated $\mathrm{C}-14$ fractions. In the sole case where the data was not qualified as an upper limit, no C-14 was detected and a detection limit value was assigned. The average $\mathrm{C}-14$ values calculated for the filtered liquid and suspended solids samples were based upon detection limit and upper limit numbers. Therefore, these data should be viewed as conservative upper limits only.

Extremely limited quantities of sludge samples severely restricted the analyses that could be performed on the samples. Because of the limited analytical data available on the sludges, C-14, I-129, Sr-90, and Tc-99 activity levels had to be based upon average activity concentrations of these radionuclides in the suspended solids. This assumption is most likely very conservative, since the activity per gram of these radionuclides is probably much higher in the suspended solids than in the sludge. The net result of this assumption is therefore most likely a gross overestimation of the inventories of these four radionuclides in the sludge phases. This assumption is supported by the data shown in Table 2 which compares the average activity concentrations of several radionuclides present in both the suspended solids and the sludge.

Table 2. Comparison of Activity Levels in Suspended Solids and Sludge

\begin{tabular}{|c|c|c|c|}
\hline COI & $\begin{array}{c}\text { Suspended Solids } \\
\mu \mathbf{C i} / \mathbf{g}\end{array}$ & $\begin{array}{c}\text { Sludge } \\
\mu \mathbf{C i} / \mathbf{g}\end{array}$ & $\begin{array}{c}\text { Ratio of } \\
\text { Susp Solids/Sludge }\end{array}$ \\
\hline $\mathrm{H}-3$ & $7.29 \mathrm{E}-01$ & $2.41 \mathrm{E}+00$ & $3.02 \mathrm{E}-01$ \\
\hline $\mathrm{Cs}-137$ & $6.22 \mathrm{E}+00$ & $1.57 \mathrm{E}+00$ & $3.96 \mathrm{E}+00$ \\
\hline $\mathrm{Pu}-238$ & $1.61 \mathrm{E}+02$ & $1.36 \mathrm{E}+01$ & $1.18 \mathrm{E}+01$ \\
\hline $\mathrm{Pu}-239$ & $9.95 \mathrm{E}+00$ & $4.69 \mathrm{E}-01$ & $2.12 \mathrm{E}+01$ \\
\hline $\mathrm{Pu}-241$ & $5.07 \mathrm{E}+01$ & $4.04 \mathrm{E}+00$ & $1.25 \mathrm{E}+01$ \\
\hline $\mathrm{U}-235$ & $3.89 \mathrm{E}-03$ & $6.19 \mathrm{E}-04$ & $6.28 \mathrm{E}+00$ \\
\hline $\mathrm{U}-238$ & $7.92 \mathrm{E}-02$ & $1.90 \mathrm{E}-02$ & $4.17 \mathrm{E}+00$ \\
\hline $\mathrm{Co}-60$ & $2.13 \mathrm{E}-02$ & $4.50 \mathrm{E}-04^{\mathrm{a}}$ & $4.73 \mathrm{E}+01$ \\
\hline $\mathrm{Np}-237$ & $1.08 \mathrm{E}-01$ & $2.38 \mathrm{E}-03$ & $4.54 \mathrm{E}+01$ \\
\hline $\mathrm{Cm}-244$ & $2.62 \mathrm{E}+02$ & $4.34 \mathrm{E}+01$ & $6.04 \mathrm{E}+00$ \\
\hline
\end{tabular}


a- This value is based upon one data point only. Co-60 was not detected in any of the other sludge samples analyzed for this constituent.

Since no data exists for the metals $(\mathrm{Cd}, \mathrm{Pb}$, and $\mathrm{Hg})$ content of the suspended solids material, their concentration can only be suggested from Table 2. Based upon the data in Table 2, the ratios of Suspended Solids to Sludge ranged from 0.302 to 47.3. The average of this ratio for the COIs listed in Table 2, excluding tritium, was $17.6 \pm 17.2$. Tritium was omitted because it is believed to be entrenched in the sludge, and is therefore, not representative of $\mathrm{Pb}, \mathrm{Hg}$, and $\mathrm{Cd}$. Thus, in order to be conservative, the $\mathrm{Pb}, \mathrm{Hg}$, and $\mathrm{Cd}$ concentrations in the suspended solids were assumed to be 50 times the concentrations of these respective metals in the sludge.

The VOC content of both the suspended solids material and sludge was based upon the filtered liquid data and corrected for the expected difference in density between the materials (e.g. sludge and suspended solids density was assumed $2 \mathrm{~g} / \mathrm{cm}^{3}$ while the liquid density was approximately $1 \mathrm{~g} / \mathrm{cm}^{3}$ ). It was also assumed that the average VOC mass per unit volume was the same for the sludge, suspended solids, and filtered liquids. It is unknown whether these assumptions are conservative or not.

\subsection{REFERENCES}

1. Information Document on the Old Solvent Tanks S1-S22 in the Old Radioactive Waste Burial Ground 643-E, WSRC-RP-96-00144, Rev. 0, Westinghouse Savannah River Company, Aiken, SC 29808 (1996).

2. Filpus-Luyckx, P., Sampling and Analysis Plan for Old Solvent Tanks S1-S22 to Address Waste Acceptance Criteria (U), WSRC-RP-97-00770, Rev. 0, Westinghouse Savannah River Company, Aiken, SC 29808 (1997).

3. Leyba, J. D., Characterization of Samples from Old Solvent Tanks S1 through S22, WSRC-RP-98-01486, Rev. 1, Westinghouse Savannah River Company, Aiken, SC 29808 (1999).

4. Mason, J., Estimation of Total Solids in Tanks S1-S22 Based on the Video Surveys, Interoffice Memorandum, April 22, 1998, Westinghouse Savannah River Company, Aiken, SC 29808 (1998).

5. Preston, A., Solvent Tank Liquid and Sludge Quantities, Electronic Mail Private Communication, December 9, 1998, Westinghouse Savannah River Company, Aiken, SC 29808 (1998).

6. Preston, A., Private Communications, December 18, 1998 and January 5, 1999, Westinghouse Savannah River Company, Aiken, SC 29808 (1998, 1999). 


\subsection{APPENDICES}

6.1 Appendix 1. COI Total Inventory of OSTs S1 through S22

6.2 Appendix 2. Solvent Tank Nuclide Inventory - Filtered Liquids

6.3 Appendix 3. Solvent Tank Nuclide Inventory - Suspended Solids

6.4 Appendix 4. Solvent Tank Nuclide Inventory - Sludges 


\begin{tabular}{|c|c|c|c|c|c|c|c|c|c|c|c|c|c|c|}
\hline & H-3 & C-14 & I-129 & Cs-137 & Pu-238 & Pu-239 & Pu-241 & Sr-90 & U-235 & U-238 & Co-60 & Tc-99 & Np-237 & $\mathrm{Cm}-244$ \\
\hline Tank \# & uCi & uCi & uCi & $\mathbf{u C i}$ & $\mathbf{u C i}$ & $\mathbf{u C i}$ & uCi & $\mathrm{uCi}$ & uCi & uCi & uCi & $\mathbf{u C i}$ & uCi & $\mathbf{u C i}$ \\
\hline 1-L & $9.18 \mathrm{E}+01$ & $1.55 \mathrm{E}+02$ & $9.00 \mathrm{E}-01$ & $5.67 \mathrm{E}+03$ & $2.88 \mathrm{E}+05$ & $6.16 \mathrm{E}+03$ & $7.09 \mathrm{E}+04$ & $2.77 \mathrm{E}+05$ & $3.79 \mathrm{E}+01$ & $8.38 \mathrm{E}+02$ & $1.34 \mathrm{E}+01$ & $8.24 \mathrm{E}+02$ & $1.02 \mathrm{E}+02$ & $9.04 \mathrm{E}+03$ \\
\hline $1-S S$ & $4.46 \mathrm{E}+01$ & $1.19 \mathrm{E}+02$ & $3.67 \mathrm{E}+02$ & $5.39 \mathrm{E}+01$ & $3.62 \mathrm{E}+03$ & $2.52 \mathrm{E}+02$ & $1.38 \mathrm{E}+03$ & $3.13 \mathrm{E}+03$ & 8.84E-02 & $2.04 \mathrm{E}+00$ & $2.43 E+01$ & $6.25 \mathrm{E}+00$ & $2.85 E+00$ & $4.58 \mathrm{E}+03$ \\
\hline $1-S$ & $8.20 \mathrm{E}+04$ & $1.04 \mathrm{E}+05$ & $4.34 \mathrm{E}+03$ & $5.36 \mathrm{E}+04$ & $4.63 \mathrm{E}+05$ & $1.60 \mathrm{E}+04$ & $1.38 \mathrm{E}+05$ & $1.54 \mathrm{E}+06$ & $2.11 \mathrm{E}+01$ & $6.47 \mathrm{E}+02$ & $1.44 \mathrm{E}+02$ & $2.62 E+03$ & $8.10 \mathrm{E}+01$ & $1.48 \mathrm{E}+06$ \\
\hline 2-L & $3.05 \mathrm{E}+02$ & $3.10 \mathrm{E}+01$ & $6.76 \mathrm{E}+01$ & $7.78 \mathrm{E}+03$ & $6.00 \mathrm{E}+03$ & $8.37 \mathrm{E}+02$ & $2.42 \mathrm{E}+03$ & $7.61 \mathrm{E}+02$ & $5.79 \mathrm{E}+00$ & $1.69 \mathrm{E}+03$ & 7.91E-01 & $4.00 \mathrm{E}+01$ & $4.36 \mathrm{E}+01$ & $1.00 \mathrm{E}+03$ \\
\hline 2-SS & $3.62 \mathrm{E}+01$ & $2.60 \mathrm{E}+02$ & $1.08 \mathrm{E}+01$ & $5.54 \mathrm{E}+01$ & $1.14 \mathrm{E}+04$ & $4.01 \mathrm{E}+02$ & $2.99 \mathrm{E}+03$ & $2.32 \mathrm{E}+02$ & $6.12 \mathrm{E}-01$ & $1.53 E+01$ & $9.33 \mathrm{E}+00$ & $4.51 E+00$ & $8.10 \mathrm{E}+00$ & $2.15 \mathrm{E}+03$ \\
\hline $2-S$ & $6.05 \mathrm{E}+05$ & $7.69 \mathrm{E}+05$ & $3.20 \mathrm{E}+04$ & $5.83 \mathrm{E}+04$ & $1.50 \mathrm{E}+07$ & $7.67 E+04$ & $3.72 \mathrm{E}+06$ & $1.54 \mathrm{E}+06$ & $8.70 \mathrm{E}+00$ & $2.07 \mathrm{E}+02$ & $3.19 \mathrm{E}+03$ & $1.93 E+04$ & $2.69 \mathrm{E}+02$ & $1.87 \mathrm{E}+07$ \\
\hline 3-L & $1.09 \mathrm{E}+03$ & $1.97 \mathrm{E}+02$ & $4.52 \mathrm{E}+00$ & $4.35 \mathrm{E}+04$ & $1.51 \mathrm{E}+04$ & $1.58 \mathrm{E}+04$ & $2.59 \mathrm{E}+04$ & $5.15 \mathrm{E}+04$ & $1.18 \mathrm{E}+02$ & $2.79 E+03$ & $1.36 \mathrm{E}+01$ & $4.74 \mathrm{E}+02$ & $2.83 \mathrm{E}+02$ & $1.64 \mathrm{E}+04$ \\
\hline 3-SS & $2.50 \mathrm{E}+02$ & $6.72 E+03$ & $1.58 \mathrm{E}+02$ & $1.76 \mathrm{E}+04$ & $7.93 \mathrm{E}+04$ & $1.91 \mathrm{E}+04$ & $2.22 \mathrm{E}+04$ & $2.55 \mathrm{E}+05$ & $1.47 \mathrm{E}+01$ & $2.99 \mathrm{E}+02$ & $1.15 \mathrm{E}+02$ & $6.48 \mathrm{E}+01$ & $1.49 \mathrm{E}+02$ & $2.08 \mathrm{E}+05$ \\
\hline 3-S & $6.74 \mathrm{E}+04$ & $8.57 \mathrm{E}+04$ & $3.57 \mathrm{E}+03$ & $4.41 \mathrm{E}+04$ & $3.81 \mathrm{E}+05$ & $1.31 \mathrm{E}+04$ & $1.13 \mathrm{E}+05$ & $1.26 \mathrm{E}+06$ & $1.73 \mathrm{E}+01$ & $5.32 \mathrm{E}+02$ & $1.18 \mathrm{E}+02$ & $2.16 \mathrm{E}+03$ & $6.66 \mathrm{E}+01$ & 1.22E+06 \\
\hline 4-L & $3.77 \mathrm{E}+03$ & $4.53 \mathrm{E}+02$ & $2.23 E+02$ & $1.38 \mathrm{E}+05$ & $2.32 \mathrm{E}+04$ & $6.36 \mathrm{E}+04$ & $1.59 \mathrm{E}+04$ & $1.71 \mathrm{E}+04$ & $2.93 \mathrm{E}+02$ & $8.69 E+03$ & $2.93 \mathrm{E}+01$ & $1.10 \mathrm{E}+03$ & $3.35 \mathrm{E}+02$ & $1.43 \mathrm{E}+03$ \\
\hline 4-SS & $3.86 \mathrm{E}+02$ & $1.54 \mathrm{E}+03$ & $3.85 \mathrm{E}+01$ & $7.18 \mathrm{E}+02$ & $9.59 \mathrm{E}+03$ & $9.50 \mathrm{E}+02$ & $2.72 \mathrm{E}+03$ & $2.72 \mathrm{E}+02$ & $1.27 \mathrm{E}+00$ & $2.59 \mathrm{E}+01$ & $2.91 E+00$ & $2.32 \mathrm{E}+01$ & $1.22 \mathrm{E}+00$ & $9.74 \mathrm{E}+01$ \\
\hline 4-S & $1.10 \mathrm{E}+03$ & $1.34 \mathrm{E}+05$ & $5.60 \mathrm{E}+03$ & $2.42 \mathrm{E}+03$ & $1.80 \mathrm{E}+03$ & $8.69 \mathrm{E}+01$ & $4.35 \mathrm{E}+03$ & $2.18 \mathrm{E}+03$ & $7.84 \mathrm{E}+00$ & $2.03 E+02$ & $1.03 E+00$ & $3.38 \mathrm{E}+03$ & $7.22 \mathrm{E}+00$ & $5.20 \mathrm{E}+02$ \\
\hline $5-L$ & $6.58 \mathrm{E}+04$ & $4.77 \mathrm{E}+02$ & $5.57 \mathrm{E}+01$ & $3.97 \mathrm{E}+06$ & $1.14 \mathrm{E}+06$ & $7.91 \mathrm{E}+04$ & $2.82 \mathrm{E}+05$ & $1.52 \mathrm{E}+04$ & 1.17E+02 & $3.17 \mathrm{E}+03$ & $5.68 \mathrm{E}+03$ & $1.88 \mathrm{E}+02$ & $1.72 \mathrm{E}+02$ & 8.67E+07 \\
\hline 5-SS & $3.23 \mathrm{E}+01$ & $1.07 \mathrm{E}+01$ & 4.44E-01 & $5.95 \mathrm{E}+00$ & $4.49 \mathrm{E}+02$ & $2.40 \mathrm{E}+01$ & $3.23 \mathrm{E}+01$ & $4.47 \mathrm{E}+00$ & $3.46 \mathrm{E}-03$ & $8.25 \mathrm{E}-02$ & 1.13E-01 & $2.68 \mathrm{E}-01$ & 7.73E-02 & 8.87E+03 \\
\hline $5-S$ & $2.04 \mathrm{E}+06$ & $2.60 \mathrm{E}+06$ & $1.08 \mathrm{E}+05$ & $1.33 \mathrm{E}+06$ & $1.15 \mathrm{E}+07$ & $3.98 \mathrm{E}+05$ & $3.42 \mathrm{E}+06$ & $3.82 \mathrm{E}+07$ & $5.25 \mathrm{E}+02$ & $1.61 \mathrm{E}+04$ & $3.58 \mathrm{E}+03$ & $6.53 \mathrm{E}+04$ & $2.02 \mathrm{E}+03$ & $3.68 \mathrm{E}+07$ \\
\hline 6-L & $5.02 \mathrm{E}+03$ & $5.54 \mathrm{E}+02$ & $1.47 \mathrm{E}+02$ & $9.01 \mathrm{E}+04$ & $1.29 \mathrm{E}+04$ & $4.74 \mathrm{E}+04$ & $2.90 \mathrm{E}+04$ & $1.03 \mathrm{E}+03$ & $3.84 \mathrm{E}+01$ & $8.92 \mathrm{E}+02$ & $1.52 \mathrm{E}+02$ & $1.70 \mathrm{E}+03$ & $2.34 \mathrm{E}+02$ & $6.43 \mathrm{E}+02$ \\
\hline 6-SS & $3.61 \mathrm{E}+02$ & $1.35 \mathrm{E}+03$ & $2.10 \mathrm{E}+03$ & $1.32 \mathrm{E}+04$ & $5.35 \mathrm{E}+05$ & $9.08 \mathrm{E}+04$ & $9.52 \mathrm{E}+04$ & $5.46 \mathrm{E}+04$ & $1.11 \mathrm{E}+02$ & $3.66 \mathrm{E}+03$ & $1.07 \mathrm{E}+02$ & $1.27 \mathrm{E}+03$ & $1.51 \mathrm{E}+02$ & $1.80 \mathrm{E}+04$ \\
\hline 6-S & $0.00 \mathrm{E}+00$ & $0.00 \mathrm{E}+00$ & $0.00 \mathrm{E}+00$ & $0.00 \mathrm{E}+00$ & $0.00 \mathrm{E}+00$ & $0.00 \mathrm{E}+00$ & $0.00 \mathrm{E}+00$ & $0.00 \mathrm{E}+00$ & $0.00 \mathrm{E}+00$ & $0.00 \mathrm{E}+00$ & $0.00 \mathrm{E}+00$ & $0.00 \mathrm{E}+00$ & $0.00 \mathrm{E}+00$ & $0.00 \mathrm{E}+00$ \\
\hline 7-L & $1.12 \mathrm{E}+03$ & $3.28 \mathrm{E}+02$ & $5.04 \mathrm{E}+01$ & $1.81 \mathrm{E}+04$ & $2.05 \mathrm{E}+05$ & $1.22 \mathrm{E}+05$ & $4.01 \mathrm{E}+05$ & $1.86 \mathrm{E}+05$ & $2.78 \mathrm{E}+02$ & $1.34 \mathrm{E}+04$ & $2.15 \mathrm{E}+01$ & $3.95 E+04$ & $8.11 \mathrm{E}+02$ & $2.62 \mathrm{E}+03$ \\
\hline 7-SS & $3.94 \mathrm{E}+02$ & $5.06 \mathrm{E}+03$ & $3.18 \mathrm{E}+02$ & $1.13 \mathrm{E}+03$ & $8.73 \mathrm{E}+03$ & $1.36 \mathrm{E}+04$ & 3.07E+04 & $1.84 \mathrm{E}+04$ & $1.00 \mathrm{E}+01$ & $3.62 \mathrm{E}+01$ & $2.29 \mathrm{E}+00$ & $1.92 \mathrm{E}+02$ & $8.95 \mathrm{E}+00$ & $1.89 \mathrm{E}+02$ \\
\hline 7-S & $2.73 E+04$ & $3.48 \mathrm{E}+04$ & $1.45 \mathrm{E}+03$ & $9.97 \mathrm{E}+02$ & $2.29 \mathrm{E}+03$ & $4.60 \mathrm{E}+03$ & $3.77 \mathrm{E}+03$ & $6.40 \mathrm{E}+03$ & $6.60 \mathrm{E}+00$ & $1.95 \mathrm{E}+02$ & $1.59 \mathrm{E}+00$ & $8.74 \mathrm{E}+02$ & $9.36 \mathrm{E}+00$ & $4.13 \mathrm{E}+02$ \\
\hline 8-L & $9.44 \mathrm{E}+02$ & $4.46 \mathrm{E}+02$ & $8.29 \mathrm{E}+01$ & $4.39 \mathrm{E}+05$ & $1.37 \mathrm{E}+04$ & $2.36 E+04$ & $2.59 \mathrm{E}+05$ & $5.00 \mathrm{E}+03$ & $1.32 \mathrm{E}+01$ & $3.13 E+02$ & $1.85 \mathrm{E}+02$ & $6.12 \mathrm{E}+02$ & $2.69 \mathrm{E}+02$ & 1.17E+03 \\
\hline 8-SS & $1.38 \mathrm{E}+02$ & $3.54 \mathrm{E}+02$ & $6.25 \mathrm{E}+01$ & $2.04 \mathrm{E}+04$ & $4.22 \mathrm{E}+04$ & $5.69 E+03$ & $4.95 \mathrm{E}+03$ & $5.40 \mathrm{E}+04$ & $1.22 \mathrm{E}-01$ & $2.65 \mathrm{E}+00$ & $3.38 \mathrm{E}+00$ & $3.78 \mathrm{E}+01$ & $7.90 \mathrm{E}+00$ & $4.42 \mathrm{E}+02$ \\
\hline 8-S & $1.09 E+05$ & $1.39 \mathrm{E}+05$ & $5.79 \mathrm{E}+03$ & $4.91 \mathrm{E}+05$ & $1.73 \mathrm{E}+04$ & $1.66 \mathrm{E}+03$ & $1.80 \mathrm{E}+03$ & $3.36 \mathrm{E}+05$ & 3.32E-01 & $7.54 \mathrm{E}+00$ & $5.54 \mathrm{E}+01$ & $3.50 \mathrm{E}+03$ & $9.49 \mathrm{E}+00$ & $7.59 \mathrm{E}+02$ \\
\hline 9-L & $0.00 \mathrm{E}+00$ & $0.00 \mathrm{E}+00$ & $0.00 \mathrm{E}+00$ & $0.00 \mathrm{E}+00$ & $0.00 \mathrm{E}+00$ & $0.00 \mathrm{E}+00$ & $0.00 \mathrm{E}+00$ & $0.00 \mathrm{E}+00$ & $0.00 \mathrm{E}+00$ & $0.00 \mathrm{E}+00$ & $0.00 \mathrm{E}+00$ & $0.00 \mathrm{E}+00$ & $0.00 \mathrm{E}+00$ & $0.00 \mathrm{E}+00$ \\
\hline 9-SS & $0.00 \mathrm{E}+00$ & $0.00 \mathrm{E}+00$ & $0.00 \mathrm{E}+00$ & $0.00 \mathrm{E}+00$ & $0.00 \mathrm{E}+00$ & $0.00 \mathrm{E}+00$ & $0.00 \mathrm{E}+00$ & $0.00 \mathrm{E}+00$ & $0.00 \mathrm{E}+00$ & $0.00 \mathrm{E}+00$ & $0.00 \mathrm{E}+00$ & $0.00 \mathrm{E}+00$ & $0.00 \mathrm{E}+00$ & $0.00 \mathrm{E}+00$ \\
\hline 9-S & $2.37 \mathrm{E}+05$ & $3.01 E+05$ & $1.25 \mathrm{E}+04$ & $1.55 \mathrm{E}+05$ & $1.34 \mathrm{E}+06$ & $4.62 \mathrm{E}+04$ & $3.97 \mathrm{E}+05$ & $4.43 \mathrm{E}+06$ & $6.09 \mathrm{E}+01$ & $1.87 \mathrm{E}+03$ & $4.16 \mathrm{E}+02$ & $7.58 \mathrm{E}+03$ & $2.34 \mathrm{E}+02$ & $4.27 \mathrm{E}+06$ \\
\hline $10-\mathrm{L}$ & $7.46 \mathrm{E}+01$ & $1.23 \mathrm{E}+01$ & $6.06 \mathrm{E}+00$ & $3.49 \mathrm{E}+04$ & $2.26 \mathrm{E}+04$ & $1.86 \mathrm{E}+03$ & $8.51 \mathrm{E}+03$ & $2.96 \mathrm{E}+03$ & $3.75 \mathrm{E}+00$ & $1.52 \mathrm{E}+02$ & $3.02 \mathrm{E}+00$ & $1.27 \mathrm{E}+02$ & $1.28 \mathrm{E}+01$ & $1.90 \mathrm{E}+03$ \\
\hline 10-SS & $6.15 \mathrm{E}+01$ & $2.58 \mathrm{E}+02$ & $1.08 \mathrm{E}+01$ & $5.24 \mathrm{E}+02$ & $1.35 \mathrm{E}+04$ & $8.39 E+02$ & $4.28 \mathrm{E}+03$ & $3.80 \mathrm{E}+03$ & $3.28 \mathrm{E}-01$ & $6.68 E+00$ & $1.79 \mathrm{E}+00$ & $6.49 \mathrm{E}+00$ & $9.14 \mathrm{E}+00$ & $2.21 E+04$ \\
\hline $10-S$ & $5.65 \mathrm{E}+05$ & $7.18 \mathrm{E}+05$ & $2.99 \mathrm{E}+04$ & $3.69 \mathrm{E}+05$ & $3.19 \mathrm{E}+06$ & $1.10 \mathrm{E}+05$ & $9.48 \mathrm{E}+05$ & $1.06 \mathrm{E}+07$ & $1.45 \mathrm{E}+02$ & $4.46 \mathrm{E}+03$ & $9.92 \mathrm{E}+02$ & $1.81 \mathrm{E}+04$ & $5.58 \mathrm{E}+02$ & $1.02 \mathrm{E}+07$ \\
\hline $11-\mathrm{L}$ & $3.31 \mathrm{E}+02$ & $1.76 \mathrm{E}+01$ & $8.65 \mathrm{E}+00$ & $5.32 \mathrm{E}+04$ & $1.36 \mathrm{E}+05$ & $2.35 \mathrm{E}+03$ & $6.17 \mathrm{E}+04$ & $9.80 \mathrm{E}+02$ & 1.09E-01 & $2.63 E+00$ & $3.42 \mathrm{E}+01$ & $1.59 \mathrm{E}+02$ & $2.68 \mathrm{E}-01$ & $2.76 \mathrm{E}+05$ \\
\hline 11-SS & $8.72 \mathrm{E}+01$ & $1.16 \mathrm{E}+01$ & 4.83E-01 & $2.43 \mathrm{E}+01$ & $9.29 \mathrm{E}+01$ & $5.65 \mathrm{E}+00$ & $3.59 \mathrm{E}+01$ & $2.11 \mathrm{E}+02$ & $2.10 \mathrm{E}-04$ & $5.69 \mathrm{E}-03$ & 3.41E-02 & 2.92E-01 & 6.44E-03 & $2.58 \mathrm{E}+02$ \\
\hline $11-S$ & 2.31E+05 & $2.94 \mathrm{E}+05$ & $1.23 \mathrm{E}+04$ & 1.51E+05 & $1.31 \mathrm{E}+06$ & $4.51 E+04$ & $3.88 \mathrm{E}+05$ & 4.33E+06 & $5.95 \mathrm{E}+01$ & $1.83 E+03$ & $4.06 \mathrm{E}+02$ & $7.40 \mathrm{E}+03$ & $2.29 \mathrm{E}+02$ & 4.17E+06 \\
\hline 12-L & $3.72 \mathrm{E}+01$ & $6.13 \mathrm{E}+00$ & $3.02 \mathrm{E}+00$ & $1.74 \mathrm{E}+04$ & $1.13 \mathrm{E}+04$ & $9.28 \mathrm{E}+02$ & $4.24 \mathrm{E}+03$ & $1.48 \mathrm{E}+03$ & $1.87 \mathrm{E}+00$ & $7.56 \mathrm{E}+01$ & $1.51 \mathrm{E}+00$ & $6.32 \mathrm{E}+01$ & $6.38 \mathrm{E}+00$ & $9.50 \mathrm{E}+02$ \\
\hline 12-SS & $3.08 \mathrm{E}+01$ & $1.29 \mathrm{E}+02$ & $5.38 \mathrm{E}+00$ & $2.62 \mathrm{E}+02$ & $6.77 \mathrm{E}+03$ & $4.20 \mathrm{E}+02$ & $2.14 \mathrm{E}+03$ & $1.90 \mathrm{E}+03$ & 1.64E-01 & $3.34 \mathrm{E}+00$ & 8.96E-01 & $3.25 \mathrm{E}+00$ & $4.57 \mathrm{E}+00$ & $1.10 \mathrm{E}+04$ \\
\hline $12-S$ & $9.11 \mathrm{E}+04$ & $1.16 \mathrm{E}+05$ & $4.83 \mathrm{E}+03$ & $5.96 \mathrm{E}+04$ & $5.15 \mathrm{E}+05$ & $1.78 \mathrm{E}+04$ & $1.53 \mathrm{E}+05$ & $1.71 \mathrm{E}+06$ & $2.34 \mathrm{E}+01$ & $7.19 E+02$ & $1.60 \mathrm{E}+02$ & $2.91 \mathrm{E}+03$ & $9.00 \mathrm{E}+01$ & $1.64 \mathrm{E}+06$ \\
\hline 13-L & $4.06 \mathrm{E}+02$ & $2.03 \mathrm{E}+02$ & $1.23 \mathrm{E}+02$ & $1.12 \mathrm{E}+05$ & $6.21 \mathrm{E}+05$ & $3.76 E+04$ & $1.68 \mathrm{E}+05$ & $2.35 \mathrm{E}+04$ & $1.11 \mathrm{E}+02$ & $2.78 \mathrm{E}+03$ & $8.59 \mathrm{E}+01$ & $2.60 \mathrm{E}+03$ & $4.06 \mathrm{E}+02$ & 1.87E+04 \\
\hline 13-SS & $4.85 \mathrm{E}+02$ & $1.39 \mathrm{E}+03$ & $2.31 \mathrm{E}+01$ & $3.53 \mathrm{E}+03$ & $2.05 \mathrm{E}+05$ & $1.19 E+04$ & $7.06 \mathrm{E}+04$ & $2.26 \mathrm{E}+04$ & $3.03 \mathrm{E}+00$ & $4.62 \mathrm{E}+01$ & $6.45 \mathrm{E}+00$ & $1.39 \mathrm{E}+01$ & $2.17 \mathrm{E}+01$ & $2.10 \mathrm{E}+03$ \\
\hline 13-S & $7.83 \mathrm{E}+05$ & $9.96 \mathrm{E}+05$ & $4.15 \mathrm{E}+04$ & $5.12 \mathrm{E}+05$ & $4.43 \mathrm{E}+06$ & $1.53 E+05$ & $1.31 \mathrm{E}+06$ & 1.47E+07 & $2.02 \mathrm{E}+02$ & $6.18 E+03$ & $1.38 \mathrm{E}+03$ & $2.51 \mathrm{E}+04$ & $7.74 \mathrm{E}+02$ & $1.41 \mathrm{E}+07$ \\
\hline 14-L & $1.21 \mathrm{E}+03$ & $4.50 \mathrm{E}+02$ & $1.32 \mathrm{E}+01$ & $5.92 \mathrm{E}+04$ & $1.50 \mathrm{E}+05$ & $1.21 \mathrm{E}+04$ & $3.31 \mathrm{E}+04$ & $8.13 \mathrm{E}+04$ & $7.06 \mathrm{E}+01$ & $5.10 \mathrm{E}+03$ & $5.81 \mathrm{E}+01$ & $1.55 \mathrm{E}+03$ & $1.11 \mathrm{E}+03$ & $9.18 \mathrm{E}+03$ \\
\hline \multirow[t]{2}{*}{ 14-SS } & $1.86 \mathrm{E}+02$ & 7.79E+02 & $5.62 \mathrm{E}+00$ & $1.34 \mathrm{E}+02$ & $6.31 \mathrm{E}+04$ & $3.99 \mathrm{E}+02$ & $7.00 \mathrm{E}+01$ & $4.23 E+04$ & $4.58 \mathrm{E}-02$ & $1.14 \mathrm{E}+00$ & $8.60 \mathrm{E}+00$ & $1.96 \mathrm{E}+01$ & $8.68 \mathrm{E}-01$ & $3.46 \mathrm{E}+04$ \\
\hline & H-3 & C-14 & I-129 & Cs-137 & Pu-238 & Pu-239 & Pu-241 & Sr-90 & U-235 & U-238 & Co-60 & Tc-99 & Np-237 & $\mathrm{Cm}-244$ \\
\hline Tank \# & uCi & uCi & uCi & $\mathbf{u C i}$ & $\mathbf{u C i}$ & $\mathbf{u C i}$ & $\mathbf{u C i}$ & $\mathrm{uCi}$ & $\mathbf{u C i}$ & $\mathbf{u C i}$ & $\mathbf{u C i}$ & $\mathbf{u C i}$ & uCi & uCi \\
\hline
\end{tabular}


Values in Bold Blue are explained below.

Most Bold Blue values were calculated using the average activity concentrations for each phase times the mass in that phase.

(Ex: $\mathrm{H}-3$ for Tank 10-L was calc'd by using the ave $\mathrm{H}-3$ activity concentration for all of the liquids for which $\mathrm{H}-3$ was measured times the volume of the Tank S10 liquid.)

C-14, I-129, Sr-90, and Tc-99 values for the sludges are based upon the suspended solids data.

VOC for sludges and suspended solids is based upon filtered liquid data and corrected for estimated density of $2 \mathrm{~g} / \mathrm{cm} 3$.

$\mathrm{Cd}, \mathrm{Pb}$, and $\mathrm{Hg}$ values for the suspended solids are based upon the sludge data (no metals or VOC data were taken for the suspended solids).

$\mathrm{C}-14$ and $\mathrm{I}-129$ values for Tank 11-L (organic liquid) are based upon average aqueous liquid data.

$\mathrm{L}-129$ value for Tank $20-\mathrm{T}-\mathrm{L}$ (organic liquid) is based upon average aqueous liquid data.

C-14, I-129, and Tc-99 values for Tank 11-SS (suspended solids in organic liquid) are based upon average suspended solids in aqueous liquid data.

C-14 value for Tank 20-T-SS (suspended solids in organic liquid) is based upon average suspended solids in aqueous liquid data.

C-14 data are based upon upper limits only. 


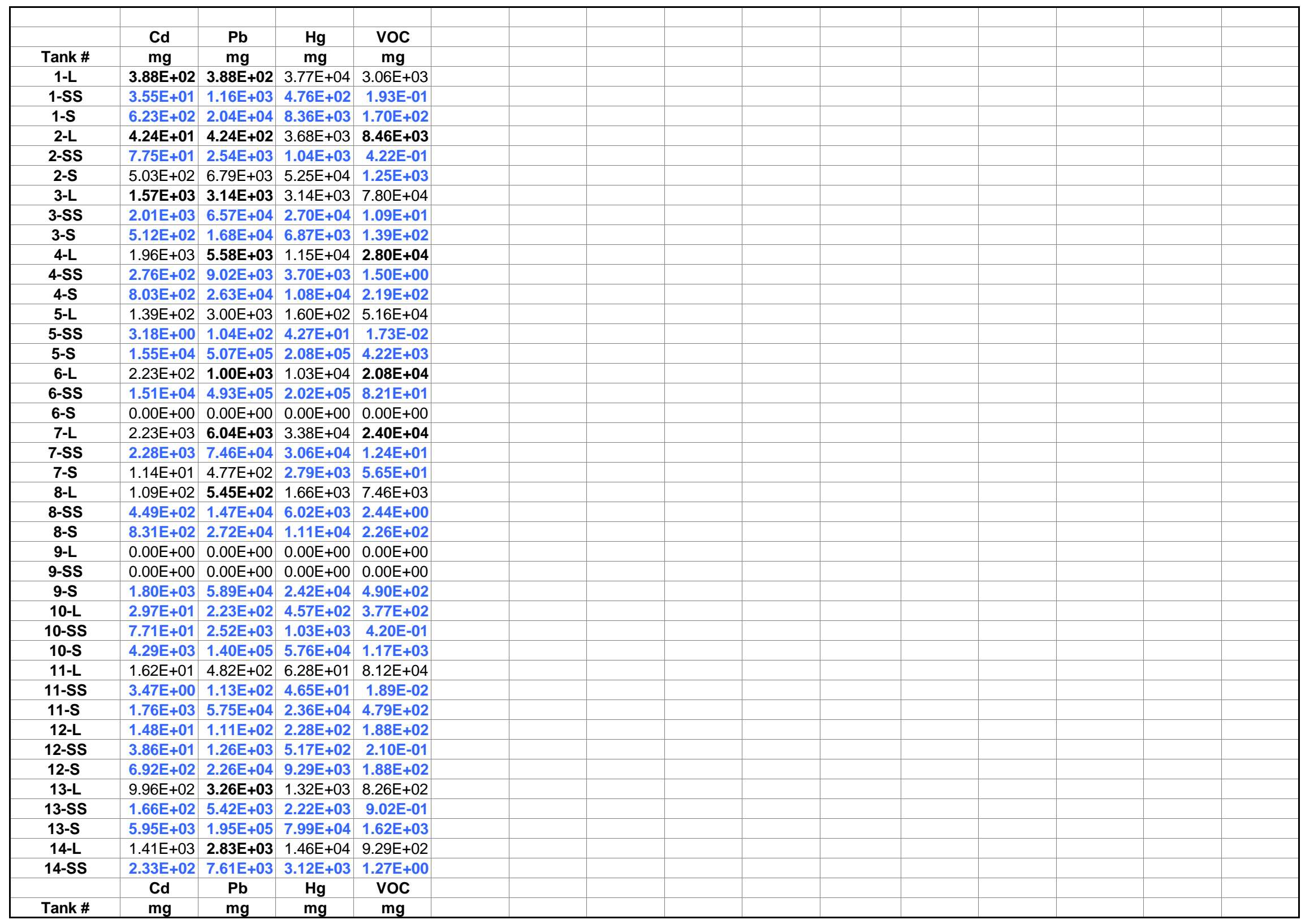




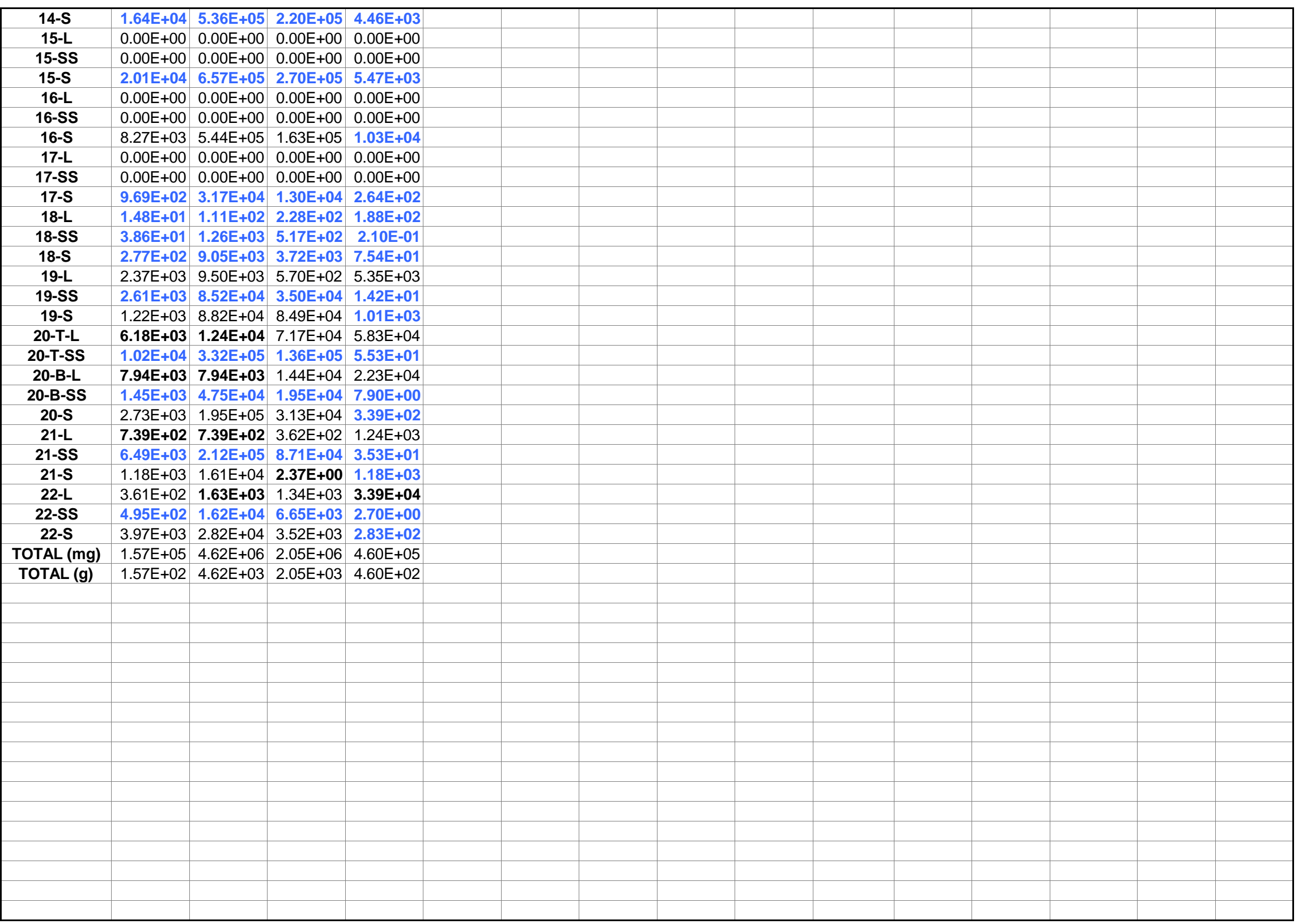




\begin{tabular}{|c|c|c|c|c|c|c|c|c|c|c|c|c|c|c|}
\hline \multicolumn{15}{|l|}{ Sludge } \\
\hline & & $(\mathrm{uCi} / \mathrm{g})$ & $(\mathrm{uCi} / \mathrm{g})$ & (uCi/g) & (uCi/g) & $(\mathrm{uCi} / \mathrm{g})$ & $(\mathrm{uCi} / \mathrm{g})$ & $(\mathrm{uCi} / \mathrm{g})$ & $(\mathrm{uCi} / \mathrm{g})$ & $(\mathrm{uCi} / \mathrm{g})$ & $(\mathrm{uCi} / \mathrm{g})$ & $(\mathrm{uCi} / \mathrm{g})$ & $(\mathrm{uCi} / \mathrm{g})$ & (uCi/g) \\
\hline & Std Dev & $2.17 \mathrm{E}+00$ & $2.87 E+00$ & 1.91E-02 & $3.73 E+00$ & $2.19 E+01$ & $3.70 \mathrm{E}-01$ & $5.80 \mathrm{E}+00$ & $5.12 \mathrm{E}+01$ & $9.80 \mathrm{E}-04$ & $2.00 \mathrm{E}-02$ & $0.00 \mathrm{E}+00$ & 5.52E-02 & $2.23 \mathrm{E}-03$ \\
\hline & Ave & $2.41 \mathrm{E}+00$ & $3.06 E+00$ & 1.28E-01 & $1.57 \mathrm{E}+00$ & $1.36 \mathrm{E}+01$ & 4.69E-01 & $4.04 \mathrm{E}+00$ & $4.51 \mathrm{E}+01$ & 6.19E-04 & 1.90E-02 & $4.23 E-03$ & 7.70E-02 & $2.38 \mathrm{E}-03$ \\
\hline Tank & Mass & H-3 & C-14 & I-129 & Cs-137 & Pu-238 & Pu-239 & Pu-241 & Sr-90 & U-235 & U-238 & Co-60 & Tc-99 & Np-237 \\
\hline$\#$ & grams & uCi & $\mathbf{u C i}$ & $\mathbf{u C i}$ & uCi & $\mathbf{u C i}$ & uCi & $\mathbf{u C i}$ & $\mathbf{u C i}$ & $\mathbf{u C i}$ & $\mathbf{u C i}$ & $\mathbf{u C i}$ & $\mathbf{u C i}$ & $\mathrm{uCi}$ \\
\hline 1 & $3.407 \mathrm{E}+04$ & $8.20 E+04$ & $1.04 \mathrm{E}+05$ & $4.34 \mathrm{E}+03$ & $5.36 \mathrm{E}+04$ & $4.63 E+05$ & $1.60 \mathrm{E}+04$ & $1.38 \mathrm{E}+05$ & $1.54 \mathrm{E}+06$ & $2.11 \mathrm{E}+01$ & $6.47 \mathrm{E}+02$ & $1.44 \mathrm{E}+02$ & $2.62 \mathrm{E}+03$ & $8.10 \mathrm{E}+01$ \\
\hline 2 & $2.514 \mathrm{E}+05$ & $6.05 E+05$ & $7.69 \mathrm{E}+05$ & $3.20 \mathrm{E}+04$ & $3.96 \mathrm{E}+05$ & $3.42 E+06$ & $1.18 \mathrm{E}+05$ & $1.01 E+06$ & $1.13 E+07$ & $1.56 \mathrm{E}+02$ & $4.77 \mathrm{E}+03$ & $1.06 \mathrm{E}+03$ & $1.93 E+04$ & $5.98 \mathrm{E}+02$ \\
\hline 3 & $2.801 E+04$ & $6.74 \mathrm{E}+04$ & $8.57 E+04$ & $3.57 \mathrm{E}+03$ & $4.41 \mathrm{E}+04$ & $3.81 E+05$ & $1.31 \mathrm{E}+04$ & $1.13 E+05$ & $1.26 \mathrm{E}+06$ & $1.73 E+01$ & $5.32 \mathrm{E}+02$ & $1.18 \mathrm{E}+02$ & $2.16 \mathrm{E}+03$ & $6.66 \mathrm{E}+01$ \\
\hline 4 & $4.391 E+04$ & $1.06 \mathrm{E}+05$ & $1.34 \mathrm{E}+05$ & $5.60 \mathrm{E}+03$ & $6.91 E+04$ & $5.97 E+05$ & $2.06 \mathrm{E}+04$ & $1.77 \mathrm{E}+05$ & $1.98 \mathrm{E}+06$ & $2.72 E+01$ & $8.34 \mathrm{E}+02$ & $1.86 \mathrm{E}+02$ & $3.38 \mathrm{E}+03$ & $1.04 \mathrm{E}+02$ \\
\hline 5 & $8.479 \mathrm{E}+05$ & $2.04 \mathrm{E}+06$ & $2.60 \mathrm{E}+06$ & $1.08 \mathrm{E}+05$ & $1.33 \mathrm{E}+06$ & $1.15 \mathrm{E}+07$ & $3.98 \mathrm{E}+05$ & $3.42 \mathrm{E}+06$ & $3.82 \mathrm{E}+07$ & $5.25 \mathrm{E}+02$ & $1.61 \mathrm{E}+04$ & $3.58 \mathrm{E}+03$ & $6.53 E+04$ & $2.02 E+03$ \\
\hline 6 & $0.000 \mathrm{E}+00$ & $0.00 \mathrm{E}+00$ & $0.00 \mathrm{E}+00$ & $0.00 \mathrm{E}+00$ & $0.00 \mathrm{E}+00$ & $0.00 \mathrm{E}+00$ & $0.00 \mathrm{E}+00$ & $0.00 \mathrm{E}+00$ & $0.00 \mathrm{E}+00$ & $0.00 \mathrm{E}+00$ & $0.00 \mathrm{E}+00$ & $0.00 \mathrm{E}+00$ & $0.00 \mathrm{E}+00$ & $0.00 \mathrm{E}+00$ \\
\hline 7 & $1.136 \mathrm{E}+04$ & $2.73 E+04$ & $3.48 E+04$ & $1.45 \mathrm{E}+03$ & $1.79 \mathrm{E}+04$ & $1.54 \mathrm{E}+05$ & $5.33 \mathrm{E}+03$ & $4.59 \mathrm{E}+04$ & $5.12 \mathrm{E}+05$ & $7.03 E+00$ & $2.16 \mathrm{E}+02$ & $4.80 \mathrm{E}+01$ & $8.74 \mathrm{E}+02$ & $2.70 \mathrm{E}+01$ \\
\hline 8 & $4.542 \mathrm{E}+04$ & $1.09 E+05$ & $1.39 \mathrm{E}+05$ & $5.79 \mathrm{E}+03$ & $7.15 E+04$ & $6.18 \mathrm{E}+05$ & $2.13 E+04$ & $1.83 E+05$ & $2.05 E+06$ & $2.81 \mathrm{E}+01$ & $8.63 E+02$ & $1.92 \mathrm{E}+02$ & $3.50 \mathrm{E}+03$ & $1.08 \mathrm{E}+02$ \\
\hline 9 & $9.842 \mathrm{E}+04$ & $2.37 E+05$ & $3.01 E+05$ & $1.25 \mathrm{E}+04$ & $1.55 \mathrm{E}+05$ & $1.34 \mathrm{E}+06$ & $4.62 \mathrm{E}+04$ & $3.97 E+05$ & $4.43 \mathrm{E}+06$ & $6.09 \mathrm{E}+01$ & $1.87 \mathrm{E}+03$ & $4.16 \mathrm{E}+02$ & $7.58 \mathrm{E}+03$ & $2.34 \mathrm{E}+02$ \\
\hline 10 & $2.347 \mathrm{E}+05$ & $5.65 E+05$ & $7.18 \mathrm{E}+05$ & $2.99 \mathrm{E}+04$ & $3.69 E+05$ & $3.19 E+06$ & $1.10 \mathrm{E}+05$ & $9.48 \mathrm{E}+05$ & $1.06 \mathrm{E}+07$ & $1.45 \mathrm{E}+02$ & $4.46 \mathrm{E}+03$ & $9.92 \mathrm{E}+02$ & $1.81 \mathrm{E}+04$ & $5.58 \mathrm{E}+02$ \\
\hline 11 & $9.615 \mathrm{E}+04$ & $2.31 E+05$ & $2.94 \mathrm{E}+05$ & $1.23 \mathrm{E}+04$ & $1.51 \mathrm{E}+05$ & $1.31 \mathrm{E}+06$ & $4.51 \mathrm{E}+04$ & $3.88 \mathrm{E}+05$ & $4.33 \mathrm{E}+06$ & $5.95 \mathrm{E}+01$ & $1.83 \mathrm{E}+03$ & $4.06 \mathrm{E}+02$ & $7.40 \mathrm{E}+03$ & $2.29 \mathrm{E}+02$ \\
\hline 12 & $3.785 \mathrm{E}+04$ & $9.11 \mathrm{E}+04$ & $1.16 \mathrm{E}+05$ & $4.83 \mathrm{E}+03$ & $5.96 \mathrm{E}+04$ & $5.15 E+05$ & $1.78 \mathrm{E}+04$ & $1.53 E+05$ & $1.71 \mathrm{E}+06$ & $2.34 \mathrm{E}+01$ & $7.19 \mathrm{E}+02$ & $1.60 \mathrm{E}+02$ & $2.91 E+03$ & $9.00 \mathrm{E}+01$ \\
\hline 13 & $3.255 \mathrm{E}+05$ & $7.83 E+05$ & $9.96 \mathrm{E}+05$ & $4.15 \mathrm{E}+04$ & $5.12 \mathrm{E}+05$ & $4.43 E+06$ & $1.53 \mathrm{E}+05$ & $1.31 \mathrm{E}+06$ & $1.47 \mathrm{E}+07$ & $2.02 E+02$ & $6.18 \mathrm{E}+03$ & $1.38 \mathrm{E}+03$ & $2.51 E+04$ & $7.74 \mathrm{E}+02$ \\
\hline 14 & $8.964 \mathrm{E}+05$ & $2.16 \mathrm{E}+06$ & $2.74 \mathrm{E}+06$ & $1.14 \mathrm{E}+05$ & $1.41 \mathrm{E}+06$ & $1.22 \mathrm{E}+07$ & $4.20 \mathrm{E}+05$ & $3.62 E+06$ & $4.04 \mathrm{E}+07$ & $5.55 \mathrm{E}+02$ & $1.70 \mathrm{E}+04$ & $3.79 \mathrm{E}+03$ & $6.90 \mathrm{E}+04$ & $2.13 E+03$ \\
\hline 15 & $1.099 \mathrm{E}+06$ & $2.64 \mathrm{E}+06$ & $3.36 \mathrm{E}+06$ & $1.40 \mathrm{E}+05$ & $1.73 E+06$ & $1.49 \mathrm{E}+07$ & $5.15 \mathrm{E}+05$ & $4.44 \mathrm{E}+06$ & $4.95 \mathrm{E}+07$ & $6.80 \mathrm{E}+02$ & $2.09 E+04$ & $4.64 \mathrm{E}+03$ & $8.46 \mathrm{E}+04$ & $2.61 E+03$ \\
\hline 16 & $2.067 \mathrm{E}+06$ & $4.97 E+06$ & $6.33 \mathrm{E}+06$ & $2.64 \mathrm{E}+05$ & $3.25 E+06$ & $2.81 E+07$ & $9.69 \mathrm{E}+05$ & $8.35 E+06$ & $9.31 \mathrm{E}+07$ & $1.28 \mathrm{E}+03$ & $3.92 E+04$ & $8.74 \mathrm{E}+03$ & $1.59 \mathrm{E}+05$ & $4.92 E+03$ \\
\hline 17 & $5.300 \mathrm{E}+04$ & $1.28 \mathrm{E}+05$ & $1.62 \mathrm{E}+05$ & $6.76 \mathrm{E}+03$ & $8.34 \mathrm{E}+04$ & $7.20 \mathrm{E}+05$ & $2.49 \mathrm{E}+04$ & $2.14 \mathrm{E}+05$ & $2.39 \mathrm{E}+06$ & $3.28 \mathrm{E}+01$ & $1.01 E+03$ & $2.24 \mathrm{E}+02$ & $4.08 \mathrm{E}+03$ & $1.26 \mathrm{E}+02$ \\
\hline 18 & $1.514 \mathrm{E}+04$ & $3.64 \mathrm{E}+04$ & $4.63 \mathrm{E}+04$ & 1.93E+03 & $2.38 E+04$ & $2.06 \mathrm{E}+05$ & $7.10 \mathrm{E}+03$ & $6.11 E+04$ & $6.82 \mathrm{E}+05$ & $9.37 \mathrm{E}+00$ & $2.88 \mathrm{E}+02$ & $6.40 \mathrm{E}+01$ & $1.17 \mathrm{E}+03$ & $3.60 \mathrm{E}+01$ \\
\hline 19 & $2.037 \mathrm{E}+05$ & $4.90 \mathrm{E}+05$ & $6.23 E+05$ & $2.60 \mathrm{E}+04$ & $3.20 E+05$ & $2.77 E+06$ & $9.55 \mathrm{E}+04$ & $8.22 \mathrm{E}+05$ & $9.18 \mathrm{E}+06$ & $1.26 \mathrm{E}+02$ & $3.87 E+03$ & $8.61 \mathrm{E}+02$ & $1.57 \mathrm{E}+04$ & $4.84 \mathrm{E}+02$ \\
\hline 20 & $6.814 \mathrm{E}+04$ & $1.64 \mathrm{E}+05$ & $2.09 \mathrm{E}+05$ & $8.69 \mathrm{E}+03$ & $1.07 E+05$ & $9.26 \mathrm{E}+05$ & $3.20 \mathrm{E}+04$ & $2.75 E+05$ & $3.07 \mathrm{E}+06$ & $4.22 \mathrm{E}+01$ & $1.29 \mathrm{E}+03$ & $2.88 \mathrm{E}+02$ & $5.25 \mathrm{E}+03$ & $1.62 \mathrm{E}+02$ \\
\hline 21 & $2.370 \mathrm{E}+05$ & $5.70 \mathrm{E}+05$ & $7.25 \mathrm{E}+05$ & $3.02 E+04$ & $3.73 E+05$ & $3.22 \mathrm{E}+06$ & $1.11 \mathrm{E}+05$ & $9.57 \mathrm{E}+05$ & $1.07 \mathrm{E}+07$ & $1.47 \mathrm{E}+02$ & $4.50 \mathrm{E}+03$ & $1.00 \mathrm{E}+03$ & $1.82 \mathrm{E}+04$ & $5.64 \mathrm{E}+02$ \\
\hline \multirow[t]{5}{*}{22} & $5.678 \mathrm{E}+04$ & $1.37 \mathrm{E}+05$ & $1.74 \mathrm{E}+05$ & $7.24 \mathrm{E}+03$ & $8.94 \mathrm{E}+04$ & $7.72 E+05$ & $2.66 \mathrm{E}+04$ & $2.29 E+05$ & $2.56 \mathrm{E}+06$ & $3.51 \mathrm{E}+01$ & $1.08 \mathrm{E}+03$ & $2.40 \mathrm{E}+02$ & $4.37 \mathrm{E}+03$ & $1.35 \mathrm{E}+02$ \\
\hline & & & & & & & & & & & & & & \\
\hline & & & & & & & & & & & & & & \\
\hline & & & & & & & & & & & & & & \\
\hline & & & & & & & & & & & & & & \\
\hline \multicolumn{15}{|c|}{ H-3 Only UL's obtained,therefore they were used for calculations, no MDA's used. } \\
\hline \multicolumn{15}{|c|}{\begin{tabular}{|l|l|l|l} 
Co-60 value based detection limit values. & & & \\
\end{tabular}} \\
\hline \multicolumn{15}{|c|}{ Np-237 value based upon Tanks 19,20 and 22 only. All other values were limits only. } \\
\hline \multicolumn{15}{|c|}{$\begin{array}{l}\text { Others: detected activity only used to calculate average values, no MDA's, LL's, or UL } \\
\text { C-14, I-129, Sr-90, and Tc-99 based upon suspended solids data. }\end{array}$} \\
\hline \multicolumn{15}{|c|}{ C-14, I-129, Sr-90, and Tc-99 based upon suspended solids data. } \\
\hline \multicolumn{15}{|c|}{ VOC based upon filtered liquid data and corrected for estimated density of $2 \mathrm{~g} / \mathrm{cm} 3$. } \\
\hline & & & & & & & & & & & & & & \\
\hline
\end{tabular}




\begin{tabular}{|c|c|c|c|c|c|c|c|c|c|c|c|c|c|c|}
\hline Sludge & & & & & & Sludge & & & & & & & & \\
\hline & & & & & & & & & & & & & & \\
\hline$(\mathrm{uCi} / \mathrm{g})$ & & $(\mathrm{mg} / \mathrm{kg})$ & $(\mathrm{mg} / \mathrm{kg})$ & $(\mathrm{mg} / \mathrm{kg})$ & $(\mathrm{mg} / \mathrm{kg})$ & & & & & & & & & \\
\hline $5.34 \mathrm{E}+01$ & Std Dev & $2.66 \mathrm{E}+01$ & $1.01 \mathrm{E}+03$ & $1.86 \mathrm{E}+02$ & $8.33 E+00$ & & $\mathrm{H}-3$ & Cs-137 & Pu-238 & Pu-239 & Pu-241 & U-235 & U-238 & Co-60 \\
\hline $4.34 \mathrm{E}+01$ & Ave & $1.83 \mathrm{E}+01$ & $5.98 \mathrm{E}+02$ & $2.45 E+02$ & $4.98 \mathrm{E}+00$ & Tank & uCi/g & $\mathrm{uCi} / \mathrm{g}$ & $\mathrm{uCi} / \mathrm{g}$ & $\mathrm{uCi} / \mathrm{g}$ & uCi/g & uCi/g & $\mathrm{uCi} / \mathrm{g}$ & uCi/g \\
\hline $\mathrm{Cm-244}$ & Tank & Cd & $\mathrm{Pb}$ & $\mathrm{Hg}$ & VOC & 2 & & $2.32 \mathrm{E}-01$ & $5.95 E+01$ & & $1.48 \mathrm{E}+01$ & 3.46E-05 & 8.24E-04 & $1.27 E-02$ \\
\hline $\mathbf{u C i}$ & $\#$ & $\mathrm{mg}$ & $\mathrm{mg}$ & $\mathrm{mg}$ & mg & 7 & & 8.78E-02 & 2.02E-01 & 4.05E-01 & 3.32E-01 & 5.81E-04 & 1.72E-02 & 1.40E-04 \\
\hline $1.48 \mathrm{E}+06$ & 1 & $6.23 \mathrm{E}+02$ & $2.04 E+04$ & $8.36 \mathrm{E}+03$ & $1.70 \mathrm{E}+02$ & 8 & & $1.08 \mathrm{E}+01$ & & 3.66E-02 & & 7.30E-06 & 1.66E-04 & 1.22E-03 \\
\hline $1.09 \mathrm{E}+07$ & 2 & $4.60 \mathrm{E}+03$ & $1.50 \mathrm{E}+05$ & $6.17 \mathrm{E}+04$ & $1.25 \mathrm{E}+03$ & 16 & & 1.11E-01 & 7.39E-01 & 7.16E-01 & & 8.87E-04 & $2.54 \mathrm{E}-02$ & $1.14 E-03$ \\
\hline $1.22 \mathrm{E}+06$ & 3 & $5.12 \mathrm{E}+02$ & $1.68 \mathrm{E}+04$ & $6.87 \mathrm{E}+03$ & $1.39 \mathrm{E}+02$ & 19 & $3.36 \mathrm{E}+00$ & $2.80 \mathrm{E}-01$ & $2.40 \mathrm{E}+01$ & $9.68 \mathrm{E}-01$ & $6.58 \mathrm{E}+00$ & $2.90 \mathrm{E}-03$ & 4.13E-02 & $8.96 E-03$ \\
\hline $1.91 \mathrm{E}+06$ & 4 & $8.03 \mathrm{E}+02$ & $2.63 \mathrm{E}+04$ & $1.08 \mathrm{E}+04$ & $2.19 \mathrm{E}+02$ & 20 & $4.95 \mathrm{E}+00$ & $6.71 \mathrm{E}-01$ & $5.77 \mathrm{E}+00$ & $8.15 \mathrm{E}-01$ & $1.79 \mathrm{E}+00$ & $5.00 \mathrm{E}-04$ & 4.77E-02 & $3.42 E-03$ \\
\hline $3.68 \mathrm{E}+07$ & 5 & $1.55 \mathrm{E}+04$ & $5.07 \mathrm{E}+05$ & $2.08 \mathrm{E}+05$ & $4.22 \mathrm{E}+03$ & 21 & $1.22 \mathrm{E}-01$ & $1.99 \mathrm{E}-02$ & $3.16 \mathrm{E}-01$ & 5.99E-02 & $9.50 \mathrm{E}-02$ & $1.23 \mathrm{E}-05$ & $3.38 \mathrm{E}-04$ & $2.01 E-03$ \\
\hline $0.00 \mathrm{E}+00$ & 6 & $0.00 \mathrm{E}+00$ & $0.00 \mathrm{E}+00$ & $0.00 \mathrm{E}+00$ & $0.00 \mathrm{E}+00$ & 22 & $1.19 \mathrm{E}+00$ & 3.87E-01 & $4.64 \mathrm{E}+00$ & $2.82 \mathrm{E}-01$ & 6.31E-01 & 3.01E-05 & & $4.50 \mathrm{E}-04$ \\
\hline $4.93 \mathrm{E}+05$ & 7 & $2.08 \mathrm{E}+02$ & $6.79 E+03$ & $2.79 \mathrm{E}+03$ & $5.65 \mathrm{E}+01$ & Average & $2.41 \mathrm{E}+00$ & $1.57 \mathrm{E}+00$ & $1.36 \mathrm{E}+01$ & 4.69E-01 & $4.04 \mathrm{E}+00$ & $6.19 \mathrm{E}-04$ & 1.90E-02 & $4.50 \mathrm{E}-04$ \\
\hline $1.97 \mathrm{E}+06$ & 8 & $8.31 \mathrm{E}+02$ & $2.72 E+04$ & $1.11 \mathrm{E}+04$ & $2.26 \mathrm{E}+02$ & Std Dev & $2.17 \mathrm{E}+00$ & $3.73 E+00$ & $2.19 E+01$ & $3.70 \mathrm{E}-01$ & $5.80 \mathrm{E}+00$ & $9.80 \mathrm{E}-04$ & $2.00 \mathrm{E}-02$ & $0.00 \mathrm{E}+00$ \\
\hline $4.27 \mathrm{E}+06$ & 9 & $1.80 \mathrm{E}+03$ & $5.89 E+04$ & $2.42 E+04$ & $4.90 \mathrm{E}+02$ & DL Ave & & & & & & & & $4.23 \mathrm{E}-03$ \\
\hline $1.02 \mathrm{E}+07$ & 10 & $4.29 \mathrm{E}+03$ & $1.40 \mathrm{E}+05$ & $5.76 \mathrm{E}+04$ & $1.17 \mathrm{E}+03$ & & & & & & & & & \\
\hline $4.17 \mathrm{E}+06$ & 11 & $1.76 \mathrm{E}+03$ & $5.75 E+04$ & $2.36 \mathrm{E}+04$ & $4.79 \mathrm{E}+02$ & \multicolumn{5}{|c|}{ Values in bold black represent detection limit values } & & & & \\
\hline $1.64 \mathrm{E}+06$ & 12 & $6.92 \mathrm{E}+02$ & $2.26 \mathrm{E}+04$ & $9.29 E+03$ & $1.88 \mathrm{E}+02$ & \multicolumn{3}{|c|}{$\mathrm{DL} A v e=$ detection limit average } & & & & & & \\
\hline $1.41 \mathrm{E}+07$ & 13 & $5.95 \mathrm{E}+03$ & $1.95 \mathrm{E}+05$ & $7.99 \mathrm{E}+04$ & $1.62 \mathrm{E}+03$ & & & & & & & & & \\
\hline $3.89 \mathrm{E}+07$ & 14 & $1.64 \mathrm{E}+04$ & $5.36 \mathrm{E}+05$ & $2.20 \mathrm{E}+05$ & $4.46 \mathrm{E}+03$ & & & & & & & & & \\
\hline $4.77 \mathrm{E}+07$ & 15 & $2.01 \mathrm{E}+04$ & $6.57 \mathrm{E}+05$ & $2.70 \mathrm{E}+05$ & $5.47 \mathrm{E}+03$ & & & & & & & & & \\
\hline $8.97 \mathrm{E}+07$ & 16 & $3.78 \mathrm{E}+04$ & $1.24 \mathrm{E}+06$ & $5.07 \mathrm{E}+05$ & $1.03 \mathrm{E}+04$ & & & & & & & & & \\
\hline $2.30 \mathrm{E}+06$ & 17 & $9.69 \mathrm{E}+02$ & $3.17 \mathrm{E}+04$ & $1.30 \mathrm{E}+04$ & $2.64 \mathrm{E}+02$ & & & & & & & & & \\
\hline $6.57 \mathrm{E}+05$ & 18 & $2.77 \mathrm{E}+02$ & $9.05 \mathrm{E}+03$ & $3.72 \mathrm{E}+03$ & $7.54 \mathrm{E}+01$ & & & & & & & & & \\
\hline $8.84 \mathrm{E}+06$ & 19 & $3.72 \mathrm{E}+03$ & $1.22 \mathrm{E}+05$ & $5.00 \mathrm{E}+04$ & $1.01 \mathrm{E}+03$ & & & & & & & & & \\
\hline $2.96 \mathrm{E}+06$ & 20 & $1.25 \mathrm{E}+03$ & $4.07 \mathrm{E}+04$ & $1.67 \mathrm{E}+04$ & $3.39 \mathrm{E}+02$ & & & & & & & & & \\
\hline $1.03 \mathrm{E}+07$ & 21 & $4.33 \mathrm{E}+03$ & $1.42 \mathrm{E}+05$ & $5.82 \mathrm{E}+04$ & $1.18 \mathrm{E}+03$ & & & & & & & & & \\
\hline $2.47 \mathrm{E}+06$ & 22 & $1.04 \mathrm{E}+03$ & $3.40 \mathrm{E}+04$ & $1.39 \mathrm{E}+04$ & $2.83 \mathrm{E}+02$ & & & & & & & & & \\
\hline & & & & & & & & & & & & & & \\
\hline & & & & & & & & & & & & & & \\
\hline & & & & & & & & & & & & & & \\
\hline & & & & & & & & & & & & & & \\
\hline & & & & & & & & & & & & & & \\
\hline & & & & & & & & & & & & & & \\
\hline & & & & & & & & & & & & & & \\
\hline & & & & & & & & & & & & & & \\
\hline & & & & & & & & & & & & & & \\
\hline & & & & & & & & & & & & & & \\
\hline & & & & & & & & & & & & & & \\
\hline
\end{tabular}




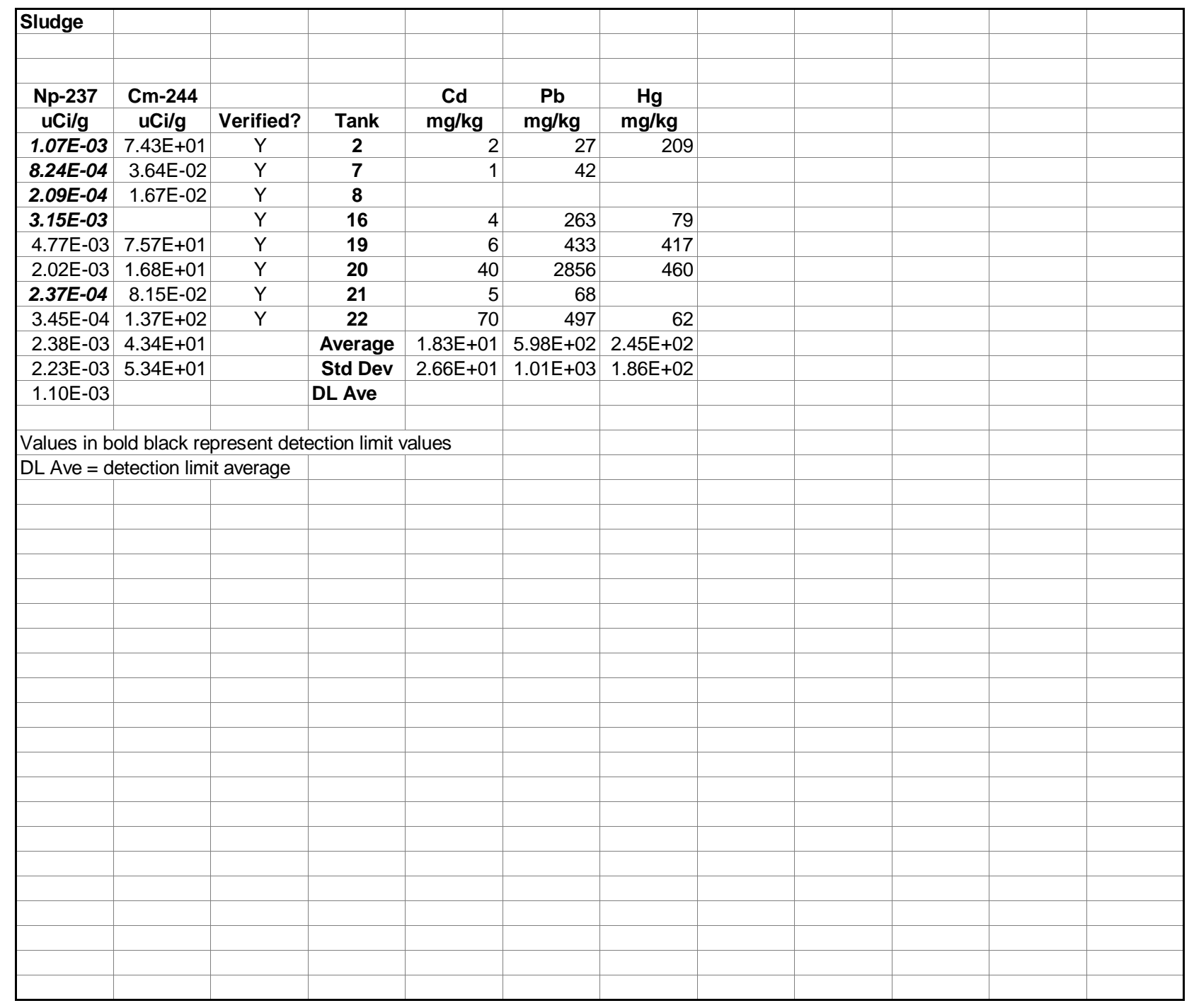




\begin{tabular}{|c|c|c|c|c|c|c|c|c|c|c|c|c|c|c|}
\hline \multicolumn{15}{|c|}{ Filtered Liquids } \\
\hline & & (uCi/mL) & (uCi/mL) & (uCi/mL) & (uCi/mL) & (uCi/mL) & (uCi/mL) & (uCi/mL) & (uCi/mL) & (uCi/mL) & (uCi/mL) & (uCi/mL) & $(\mathrm{uCi} / \mathrm{mL})$ & (uCi/mL) \\
\hline & Std Dev & 1.67E-03 & 2.14E-04 & 7.97E-05 & $3.02 \mathrm{E}+00$ & $9.40 \mathrm{E}-01$ & 7.87E-02 & $2.48 \mathrm{E}-01$ & $1.70 \mathrm{E}-01$ & 9.55E-05 & $3.46 \mathrm{E}-03$ & $1.07 \mathrm{E}-04$ & 8.54E-03 & $2.54 \mathrm{E}-04$ \\
\hline & Ave & 1.97E-03 & $3.24 \mathrm{E}-04$ & 1.60E-04 & 9.21E-01 & 5.97E-01 & 4.91E-02 & 2.24E-01 & 7.81E-02 & 9.89E-05 & 4.00E-03 & 7.98E-05 & 3.34E-03 & 3.38E-04 \\
\hline Tank & Volume & H-3 & C-14 & I-129 & Cs-137 & Pu-238 & Pu-239 & Pu-241 & Sr-90 & U-235 & U-238 & Co-60 & Tc-99 & Np-237 \\
\hline$\#$ & $\mathrm{~mL}$ & $\mathbf{u C i}$ & $\mathbf{u C i}$ & $\mathbf{u C i}$ & $\mathbf{u C i}$ & $\mathbf{u C i}$ & $\mathbf{u C i}$ & $\mathbf{u C i}$ & uCi & $\mathbf{u C i}$ & $\mathbf{u C i}$ & $\mathbf{u C i}$ & $\mathbf{u C i}$ & uCi \\
\hline 1 & $4.433 \mathrm{E}+05$ & $8.73 \mathrm{E}+02$ & $1.44 \mathrm{E}+02$ & $7.08 \mathrm{E}+01$ & $4.08 \mathrm{E}+05$ & $2.65 \mathrm{E}+05$ & $2.18 \mathrm{E}+04$ & $9.95 \mathrm{E}+04$ & $3.46 \mathrm{E}+04$ & $4.39 \mathrm{E}+01$ & $1.77 \mathrm{E}+03$ & $3.54 \mathrm{E}+01$ & $1.48 \mathrm{E}+03$ & $1.50 \mathrm{E}+02$ \\
\hline 2 & $4.228 \mathrm{E}+05$ & $8.32 \mathrm{E}+02$ & $1.37 \mathrm{E}+02$ & $6.76 \mathrm{E}+01$ & $3.89 \mathrm{E}+05$ & $2.53 \mathrm{E}+05$ & $2.08 \mathrm{E}+04$ & $9.49 \mathrm{E}+04$ & $3.30 \mathrm{E}+04$ & $4.18 \mathrm{E}+01$ & $1.69 \mathrm{E}+03$ & $3.37 \mathrm{E}+01$ & $1.41 \mathrm{E}+03$ & $1.43 E+02$ \\
\hline 3 & $1.506 \mathrm{E}+06$ & $2.97 \mathrm{E}+03$ & $4.89 \mathrm{E}+02$ & $2.41 \mathrm{E}+02$ & $1.39 E+06$ & $9.00 \mathrm{E}+05$ & $7.40 \mathrm{E}+04$ & $3.38 \mathrm{E}+05$ & $1.18 \mathrm{E}+05$ & $1.49 \mathrm{E}+02$ & $6.03 \mathrm{E}+03$ & $1.20 \mathrm{E}+02$ & $5.03 E+03$ & $5.09 E+02$ \\
\hline 4 & $1.398 \mathrm{E}+06$ & $2.75 \mathrm{E}+03$ & $4.53 \mathrm{E}+02$ & $2.23 E+02$ & $1.29 E+06$ & $8.35 \mathrm{E}+05$ & $6.86 \mathrm{E}+04$ & $3.14 \mathrm{E}+05$ & $1.09 \mathrm{E}+05$ & $1.38 \mathrm{E}+02$ & $5.59 \mathrm{E}+03$ & $1.12 \mathrm{E}+02$ & $4.67 \mathrm{E}+03$ & $4.72 \mathrm{E}+02$ \\
\hline 5 & $3.483 \mathrm{E}+05$ & $6.86 \mathrm{E}+02$ & $1.13 \mathrm{E}+02$ & $5.57 \mathrm{E}+01$ & $3.21 \mathrm{E}+05$ & $2.08 \mathrm{E}+05$ & $1.71 \mathrm{E}+04$ & $7.82 \mathrm{E}+04$ & $2.72 \mathrm{E}+04$ & $3.45 \mathrm{E}+01$ & $1.39 \mathrm{E}+03$ & $2.78 \mathrm{E}+01$ & $1.16 \mathrm{E}+03$ & $1.18 \mathrm{E}+02$ \\
\hline 6 & $1.042 \mathrm{E}+06$ & $2.05 \mathrm{E}+03$ & $3.38 \mathrm{E}+02$ & $1.67 \mathrm{E}+02$ & $9.60 \mathrm{E}+05$ & $6.22 \mathrm{E}+05$ & $5.12 \mathrm{E}+04$ & $2.34 \mathrm{E}+05$ & $8.13 \mathrm{E}+04$ & $1.03 \mathrm{E}+02$ & $4.17 \mathrm{E}+03$ & $8.32 \mathrm{E}+01$ & $3.48 \mathrm{E}+03$ & $3.52 \mathrm{E}+02$ \\
\hline 7 & $1.200 \mathrm{E}+06$ & $2.36 \mathrm{E}+03$ & $3.89 \mathrm{E}+02$ & $1.92 \mathrm{E}+02$ & $1.11 \mathrm{E}+06$ & $7.17 \mathrm{E}+05$ & $5.89 E+04$ & $2.69 \mathrm{E}+05$ & $9.37 \mathrm{E}+04$ & $1.19 \mathrm{E}+02$ & $4.80 \mathrm{E}+03$ & $9.58 \mathrm{E}+01$ & $4.01 \mathrm{E}+03$ & $4.05 \mathrm{E}+02$ \\
\hline 8 & $5.186 \mathrm{E}+05$ & $1.02 \mathrm{E}+03$ & $1.68 \mathrm{E}+02$ & $8.29 \mathrm{E}+01$ & $4.78 \mathrm{E}+05$ & $3.10 \mathrm{E}+05$ & $2.55 E+04$ & $1.16 \mathrm{E}+05$ & $4.05 \mathrm{E}+04$ & $5.13 \mathrm{E}+01$ & $2.08 \mathrm{E}+03$ & $4.14 \mathrm{E}+01$ & $1.73 \mathrm{E}+03$ & $1.75 \mathrm{E}+02$ \\
\hline 9 & $0.000 \mathrm{E}+00$ & $0.00 \mathrm{E}+00$ & $0.00 \mathrm{E}+00$ & $0.00 \mathrm{E}+00$ & $0.00 \mathrm{E}+00$ & $0.00 \mathrm{E}+00$ & $0.00 \mathrm{E}+00$ & $0.00 \mathrm{E}+00$ & $0.00 \mathrm{E}+00$ & $0.00 \mathrm{E}+00$ & $0.00 \mathrm{E}+00$ & $0.00 \mathrm{E}+00$ & $0.00 \mathrm{E}+00$ & $0.00 \mathrm{E}+00$ \\
\hline 10 & $3.790 \mathrm{E}+04$ & $7.46 \mathrm{E}+01$ & $1.23 \mathrm{E}+01$ & $6.06 \mathrm{E}+00$ & $3.49 E+04$ & $2.26 \mathrm{E}+04$ & $1.86 \mathrm{E}+03$ & $8.51 \mathrm{E}+03$ & $2.96 \mathrm{E}+03$ & $3.75 \mathrm{E}+00$ & $1.52 \mathrm{E}+02$ & $3.02 \mathrm{E}+00$ & $1.27 \mathrm{E}+02$ & $1.28 \mathrm{E}+01$ \\
\hline 11 & $5.410 \mathrm{E}+04$ & $1.07 \mathrm{E}+02$ & $1.76 \mathrm{E}+01$ & $8.65 \mathrm{E}+00$ & $4.98 \mathrm{E}+04$ & $3.23 \mathrm{E}+04$ & $2.66 \mathrm{E}+03$ & $1.21 \mathrm{E}+04$ & $4.22 \mathrm{E}+03$ & $5.35 \mathrm{E}+00$ & $2.16 \mathrm{E}+02$ & $4.32 \mathrm{E}+00$ & $1.81 \mathrm{E}+02$ & $1.83 \mathrm{E}+01$ \\
\hline 12 & $1.890 \mathrm{E}+04$ & $3.72 \mathrm{E}+01$ & $6.13 \mathrm{E}+00$ & $3.02 \mathrm{E}+00$ & $1.74 \mathrm{E}+04$ & $1.13 \mathrm{E}+04$ & $9.28 \mathrm{E}+02$ & $4.24 \mathrm{E}+03$ & $1.48 \mathrm{E}+03$ & $1.87 \mathrm{E}+00$ & $7.56 \mathrm{E}+01$ & $1.51 \mathrm{E}+00$ & $6.32 \mathrm{E}+01$ & $6.38 \mathrm{E}+00$ \\
\hline 13 & $8.264 \mathrm{E}+05$ & $1.63 \mathrm{E}+03$ & $2.68 \mathrm{E}+02$ & $1.32 \mathrm{E}+02$ & $7.61 \mathrm{E}+05$ & $4.94 \mathrm{E}+05$ & $4.06 \mathrm{E}+04$ & $1.85 \mathrm{E}+05$ & $6.45 \mathrm{E}+04$ & $8.18 \mathrm{E}+01$ & $3.31 \mathrm{E}+03$ & $6.60 \mathrm{E}+01$ & $2.76 \mathrm{E}+03$ & $2.79 \mathrm{E}+02$ \\
\hline 14 & $1.387 \mathrm{E}+06$ & $2.73 \mathrm{E}+03$ & $4.50 \mathrm{E}+02$ & $2.22 \mathrm{E}+02$ & $1.28 \mathrm{E}+06$ & $8.28 \mathrm{E}+05$ & $6.81 \mathrm{E}+04$ & $3.11 \mathrm{E}+05$ & $1.08 \mathrm{E}+05$ & $1.37 \mathrm{E}+02$ & $5.55 \mathrm{E}+03$ & $1.11 \mathrm{E}+02$ & $4.64 \mathrm{E}+03$ & $4.68 \mathrm{E}+02$ \\
\hline 15 & $0.000 \mathrm{E}+00$ & $0.00 \mathrm{E}+00$ & $0.00 \mathrm{E}+00$ & $0.00 \mathrm{E}+00$ & $0.00 \mathrm{E}+00$ & $0.00 \mathrm{E}+00$ & $0.00 \mathrm{E}+00$ & $0.00 \mathrm{E}+00$ & $0.00 \mathrm{E}+00$ & $0.00 \mathrm{E}+00$ & $0.00 \mathrm{E}+00$ & $0.00 \mathrm{E}+00$ & $0.00 \mathrm{E}+00$ & $0.00 \mathrm{E}+00$ \\
\hline 16 & $0.000 \mathrm{E}+00$ & $0.00 \mathrm{E}+00$ & $0.00 \mathrm{E}+00$ & $0.00 \mathrm{E}+00$ & $0.00 \mathrm{E}+00$ & $0.00 \mathrm{E}+00$ & $0.00 \mathrm{E}+00$ & $0.00 \mathrm{E}+00$ & $0.00 \mathrm{E}+00$ & $0.00 \mathrm{E}+00$ & $0.00 \mathrm{E}+00$ & $0.00 \mathrm{E}+00$ & $0.00 \mathrm{E}+00$ & $0.00 \mathrm{E}+00$ \\
\hline 17 & $0.000 \mathrm{E}+00$ & $0.00 \mathrm{E}+00$ & $0.00 \mathrm{E}+00$ & $0.00 \mathrm{E}+00$ & $0.00 \mathrm{E}+00$ & $0.00 \mathrm{E}+00$ & $0.00 \mathrm{E}+00$ & $0.00 \mathrm{E}+00$ & $0.00 \mathrm{E}+00$ & $0.00 \mathrm{E}+00$ & $0.00 \mathrm{E}+00$ & $0.00 \mathrm{E}+00$ & $0.00 \mathrm{E}+00$ & $0.00 \mathrm{E}+00$ \\
\hline 18 & $1.890 \mathrm{E}+04$ & $3.72 \mathrm{E}+01$ & $6.13 \mathrm{E}+00$ & $3.02 \mathrm{E}+00$ & $1.74 \mathrm{E}+04$ & $1.13 \mathrm{E}+04$ & $9.28 \mathrm{E}+02$ & $4.24 \mathrm{E}+03$ & $1.48 \mathrm{E}+03$ & $1.87 \mathrm{E}+00$ & $7.56 \mathrm{E}+01$ & $1.51 \mathrm{E}+00$ & $6.32 \mathrm{E}+01$ & $6.38 \mathrm{E}+00$ \\
\hline $19-0$ & $2.171 \mathrm{E}+06$ & $4.27 \mathrm{E}+03$ & $7.04 \mathrm{E}+02$ & $3.47 \mathrm{E}+02$ & $2.00 \mathrm{E}+06$ & $1.30 \mathrm{E}+06$ & $1.07 \mathrm{E}+05$ & $4.87 \mathrm{E}+05$ & $1.69 \mathrm{E}+05$ & $2.15 \mathrm{E}+02$ & $8.69 \mathrm{E}+03$ & $1.73 \mathrm{E}+02$ & $7.25 \mathrm{E}+03$ & $7.33 \mathrm{E}+02$ \\
\hline 20-T & $7.552 \mathrm{E}+06$ & $1.49 \mathrm{E}+04$ & $2.45 \mathrm{E}+03$ & $1.21 \mathrm{E}+03$ & $6.95 \mathrm{E}+06$ & $4.51 \mathrm{E}+06$ & $3.71 \mathrm{E}+05$ & $1.70 \mathrm{E}+06$ & $5.89 \mathrm{E}+05$ & $7.47 \mathrm{E}+02$ & $3.02 \mathrm{E}+04$ & $6.03 \mathrm{E}+02$ & $2.52 \mathrm{E}+04$ & $2.55 \mathrm{E}+03$ \\
\hline 20-B & $7.552 \mathrm{E}+06$ & $1.49 \mathrm{E}+04$ & $2.45 \mathrm{E}+03$ & $1.21 \mathrm{E}+03$ & $6.95 \mathrm{E}+06$ & $4.51 \mathrm{E}+06$ & $3.71 \mathrm{E}+05$ & $1.70 \mathrm{E}+06$ & $5.89 \mathrm{E}+05$ & $7.47 \mathrm{E}+02$ & $3.02 \mathrm{E}+04$ & $6.03 \mathrm{E}+02$ & $2.52 \mathrm{E}+04$ & $2.55 \mathrm{E}+03$ \\
\hline 21 & $6.905 \mathrm{E}+05$ & $1.36 \mathrm{E}+03$ & $2.24 \mathrm{E}+02$ & $1.10 \mathrm{E}+02$ & $6.36 \mathrm{E}+05$ & $4.12 \mathrm{E}+05$ & $3.39 E+04$ & $1.55 \mathrm{E}+05$ & $5.39 \mathrm{E}+04$ & $6.83 \mathrm{E}+01$ & $2.76 \mathrm{E}+03$ & $5.51 \mathrm{E}+01$ & $2.31 \mathrm{E}+03$ & $2.33 \mathrm{E}+02$ \\
\hline 22 & $1.694 \mathrm{E}+06$ & $3.34 \mathrm{E}+03$ & $5.50 \mathrm{E}+02$ & $2.71 \mathrm{E}+02$ & $1.56 \mathrm{E}+06$ & $1.01 \mathrm{E}+06$ & $8.32 E+04$ & $3.80 \mathrm{E}+05$ & $1.32 \mathrm{E}+05$ & $1.68 \mathrm{E}+02$ & $6.78 \mathrm{E}+03$ & $1.35 \mathrm{E}+02$ & $5.66 \mathrm{E}+03$ & $5.72 \mathrm{E}+02$ \\
\hline & & & & & & & & & & & & & & \\
\hline & & & & & & & & & & & & & & \\
\hline & & & & & & & & & & & & & & \\
\hline & & & & & & & & & & & & & & \\
\hline & & & & & & & & & & & & & & \\
\hline Others: $d$ & d activity o & y usea & & rage & & & & & & & & & & \\
\hline
\end{tabular}




\begin{tabular}{|c|c|c|c|c|c|c|c|c|c|c|c|c|c|c|c|}
\hline \multicolumn{2}{|c|}{ Filtered Liquids } & & & & & & \multicolumn{2}{|c|}{ Filtered Liquids } & & & & & & & \\
\hline$(\mathrm{uCi} / \mathrm{mL})$ & & $(\mathrm{mg} / \mathrm{kg})$ & $(\mathrm{mg} / \mathrm{kg})$ & $(\mathrm{mg} / \mathrm{kg})$ & $(\mathrm{mg} / \mathrm{kg})$ & & & & & & & & & & \\
\hline 8.87E-02 & Std Dev & 5.59E-01 & $2.67 \mathrm{E}+00$ & $2.54 \mathrm{E}+01$ & $1.67 \mathrm{E}+01$ & & & $\mathrm{H}-3$ & C-14 & $\mid-129$ & Cs-137 & Pu-238 & Pu-239 & Pu-241 & Sr-90 \\
\hline $5.02 \mathrm{E}-02$ & Ave & 7.84E-01 & $5.89 \mathrm{E}+00$ & $1.21 \mathrm{E}+01$ & $9.95 \mathrm{E}+00$ & & Tank & $\mathrm{uCi} / \mathrm{mL}$ & $\mathrm{uCi} / \mathrm{mL}$ & $\mathrm{uCi} / \mathrm{mL}$ & $\mathrm{uCi} / \mathrm{mL}$ & $\mathrm{uCi} / \mathrm{mL}$ & $\mathrm{uCi} / \mathrm{mL}$ & $\mathrm{uCi} / \mathrm{mL}$ & $\mathrm{uCi} / \mathrm{mL}$ \\
\hline $\mathrm{Cm}-244$ & Tank & Cd & $\mathbf{P b}$ & $\mathrm{Hg}$ & VOC & Density & 1 & 2.07E-04 & 3.50E-04 & & $1.28 \mathrm{E}-02$ & $6.49 \mathrm{E}-01$ & 1.39E-02 & $1.60 \mathrm{E}-01$ & $6.26 \mathrm{E}-01$ \\
\hline uCi & \# & mg & mg & $\mathrm{mg}$ & mg & $\mathrm{g} / \mathrm{cm} 3$ & 2 & 7.21E-04 & 7.34E-05 & & $1.84 \mathrm{E}-02$ & 1.42E-02 & & $5.72 \mathrm{E}-03$ & $1.80 \mathrm{E}-03$ \\
\hline $2.23 \mathrm{E}+04$ & 1 & $3.04 \mathrm{E}+02$ & $2.28 \mathrm{E}+03$ & $4.68 \mathrm{E}+03$ & $3.86 \mathrm{E}+03$ & 0.875 & 3 & 7.21E-04 & 1.31E-04 & & 2.89E-02 & $1.00 \mathrm{E}-02$ & 1.05E-02 & $1.72 \mathrm{E}-02$ & $3.42 \mathrm{E}-02$ \\
\hline $2.12 \mathrm{E}+04$ & 2 & $3.33 \mathrm{E}+02$ & $2.50 \mathrm{E}+03$ & $5.12 \mathrm{E}+03$ & $4.22 \mathrm{E}+03$ & 1.003 & 4 & $2.70 \mathrm{E}-03$ & 3.24E-04 & & 9.91E-02 & 1.66E-02 & & & $1.22 \mathrm{E}-02$ \\
\hline $7.57 \mathrm{E}+04$ & 3 & $1.23 \mathrm{E}+03$ & $9.24 \mathrm{E}+03$ & $1.89 \mathrm{E}+04$ & $1.56 \mathrm{E}+04$ & 1.042 & 5 & & & & $1.14 \mathrm{E}+01$ & $3.28 \mathrm{E}+00$ & 2.27E-01 & $8.11 \mathrm{E}-01$ & 4.37E-02 \\
\hline $7.02 \mathrm{E}+04$ & 4 & $1.18 \mathrm{E}+03$ & $8.88 \mathrm{E}+03$ & $1.82 \mathrm{E}+04$ & $1.50 \mathrm{E}+04$ & 1.079 & 6 & 4.82E-03 & 5.32E-04 & $1.41 \mathrm{E}-04$ & 8.65E-02 & 1.24E-02 & & $2.78 \mathrm{E}-02$ & 9.91E-04 \\
\hline $1.75 \mathrm{E}+04$ & 5 & $3.02 \mathrm{E}+02$ & $2.27 \mathrm{E}+03$ & $4.64 \mathrm{E}+03$ & $3.83 \mathrm{E}+03$ & 1.105 & 7 & 9.37E-04 & 2.73E-04 & $4.20 \mathrm{E}-05$ & 1.51E-02 & 1.71E-01 & $1.02 \mathrm{E}-01$ & 3.34E-01 & $1.55 \mathrm{E}-01$ \\
\hline $5.24 \mathrm{E}+04$ & 6 & $8.75 \mathrm{E}+02$ & $6.57 \mathrm{E}+03$ & $1.35 \mathrm{E}+04$ & $1.11 \mathrm{E}+04$ & 1.070 & 8 & 1.82E-03 & 8.60E-04 & & 8.47E-01 & 2.64E-02 & & $5.00 \mathrm{E}-01$ & $9.64 \mathrm{E}-03$ \\
\hline $6.03 \mathrm{E}+04$ & 7 & $1.03 E+03$ & $7.73 \mathrm{E}+03$ & $1.58 \mathrm{E}+04$ & $1.31 \mathrm{E}+04$ & 1.094 & 13 & 4.91E-04 & 2.46E-04 & 1.49E-04 & 1.36E-01 & 7.52E-01 & & 2.03E-01 & 2.84E-02 \\
\hline $2.61 \mathrm{E}+04$ & 8 & $4.28 \mathrm{E}+02$ & $3.21 E+03$ & $6.58 \mathrm{E}+03$ & $5.43 \mathrm{E}+03$ & 1.051 & 14 & 8.69E-04 & & & 4.27E-02 & $1.08 \mathrm{E}-01$ & 8.74E-03 & $2.39 \mathrm{E}-02$ & 5.86E-02 \\
\hline $0.00 \mathrm{E}+00$ & 9 & $0.00 \mathrm{E}+00$ & $0.00 \mathrm{E}+00$ & $0.00 \mathrm{E}+00$ & $0.00 \mathrm{E}+00$ & 0.000 & $19-0$ & 1.77E-03 & & $2.44 \mathrm{E}-04$ & $5.86 \mathrm{E}-02$ & $2.02 \mathrm{E}+00$ & $5.95 \mathrm{E}-03$ & $5.18 \mathrm{E}-01$ & 3.15E-02 \\
\hline $1.90 \mathrm{E}+03$ & 10 & $2.97 \mathrm{E}+01$ & $2.23 \mathrm{E}+02$ & $4.57 \mathrm{E}+02$ & $3.77 \mathrm{E}+02$ & 1.000 & 20-B & $1.35 \mathrm{E}-03$ & $2.00 \mathrm{E}-04$ & & 1.56E-02 & 5.59E-01 & 1.21E-02 & 1.39E-01 & \\
\hline $2.72 \mathrm{E}+03$ & 11 & $4.33 \mathrm{E}+01$ & $3.25 \mathrm{E}+02$ & $6.67 \mathrm{E}+02$ & $5.50 \mathrm{E}+02$ & 1.021 & 21 & $4.55 \mathrm{E}-03$ & $2.82 \mathrm{E}-04$ & & 5.63E-02 & $4.68 \mathrm{E}-01$ & $1.26 \mathrm{E}-02$ & $1.14 \mathrm{E}-01$ & 9.46E-03 \\
\hline $9.50 \mathrm{E}+02$ & 12 & $1.48 \mathrm{E}+01$ & $1.11 \mathrm{E}+02$ & $2.28 \mathrm{E}+02$ & $1.88 \mathrm{E}+02$ & 1.000 & 22 & $4.64 \mathrm{E}-03$ & 2.97E-04 & $2.23 \mathrm{E}-04$ & 7.57E-02 & $2.76 \mathrm{E}-01$ & & $6.44 \mathrm{E}-02$ & $3.25 \mathrm{E}-03$ \\
\hline $4.15 \mathrm{E}+04$ & 13 & $7.10 \mathrm{E}+02$ & $5.33 \mathrm{E}+03$ & $1.09 \mathrm{E}+04$ & $9.02 \mathrm{E}+03$ & 1.096 & Average & 1.97E-03 & $3.24 \mathrm{E}-04$ & $1.60 \mathrm{E}-04$ & $9.21 \mathrm{E}-01$ & 5.97E-01 & 4.91E-02 & $2.24 \mathrm{E}-01$ & 7.81E-02 \\
\hline $6.97 \mathrm{E}+04$ & 14 & $1.11 \mathrm{E}+03$ & $8.32 \mathrm{E}+03$ & $1.71 \mathrm{E}+04$ & $1.41 \mathrm{E}+04$ & 1.019 & Std Dev & 1.67E-03 & $2.14 \mathrm{E}-04$ & 7.97E-05 & $3.02 \mathrm{E}+00$ & $9.40 \mathrm{E}-01$ & 7.87E-02 & $2.48 \mathrm{E}-01$ & 1.70E-01 \\
\hline $0.00 \mathrm{E}+00$ & 15 & $0.00 \mathrm{E}+00$ & $0.00 \mathrm{E}+00$ & $0.00 \mathrm{E}+00$ & $0.00 \mathrm{E}+00$ & 0.000 & & & & & & & & & \\
\hline $0.00 \mathrm{E}+00$ & 16 & $0.00 \mathrm{E}+00$ & $0.00 \mathrm{E}+00$ & $0.00 \mathrm{E}+00$ & $0.00 \mathrm{E}+00$ & 0.000 & & & & & & & & & \\
\hline $0.00 \mathrm{E}+00$ & 17 & $0.00 \mathrm{E}+00$ & $0.00 \mathrm{E}+00$ & $0.00 \mathrm{E}+00$ & $0.00 \mathrm{E}+00$ & 0.000 & & & & & & & & & \\
\hline $9.50 \mathrm{E}+02$ & 18 & $1.48 \mathrm{E}+01$ & $1.11 \mathrm{E}+02$ & $2.28 \mathrm{E}+02$ & $1.88 \mathrm{E}+02$ & 1.000 & & & & & & & & & \\
\hline $1.09 \mathrm{E}+05$ & $19-0$ & $1.86 \mathrm{E}+03$ & $1.40 \mathrm{E}+04$ & $2.87 \mathrm{E}+04$ & $2.36 \mathrm{E}+04$ & 1.094 & & & & & & & & & \\
\hline $3.79 \mathrm{E}+05$ & 20-T & $4.85 \mathrm{E}+03$ & $3.64 \mathrm{E}+04$ & $7.45 \mathrm{E}+04$ & $6.15 \mathrm{E}+04$ & 0.818 & & & & & & & & & \\
\hline $3.79 \mathrm{E}+05$ & 20-B & $6.23 \mathrm{E}+03$ & $4.67 \mathrm{E}+04$ & $9.58 \mathrm{E}+04$ & $7.90 \mathrm{E}+04$ & 1.051 & & & & & & & & & \\
\hline $3.47 \mathrm{E}+04$ & 21 & $5.80 \mathrm{E}+02$ & $4.36 \mathrm{E}+03$ & $8.92 \mathrm{E}+03$ & $7.36 \mathrm{E}+03$ & 1.071 & & & & & & & & & \\
\hline $8.51 \mathrm{E}+04$ & 22 & $1.42 \mathrm{E}+03$ & $1.06 \mathrm{E}+04$ & $2.18 \mathrm{E}+04$ & $1.80 \mathrm{E}+04$ & 1.066 & & & & & & & & & \\
\hline & & & & & & & & & & & & & & & \\
\hline & & & & & & & & & & & & & & & \\
\hline & & & & & & & & & & & & & & & \\
\hline & & & & & & & & & & & & & & & \\
\hline & & & & & & & & & & & & & & & \\
\hline & & & & & & & & & & & & & & & \\
\hline
\end{tabular}




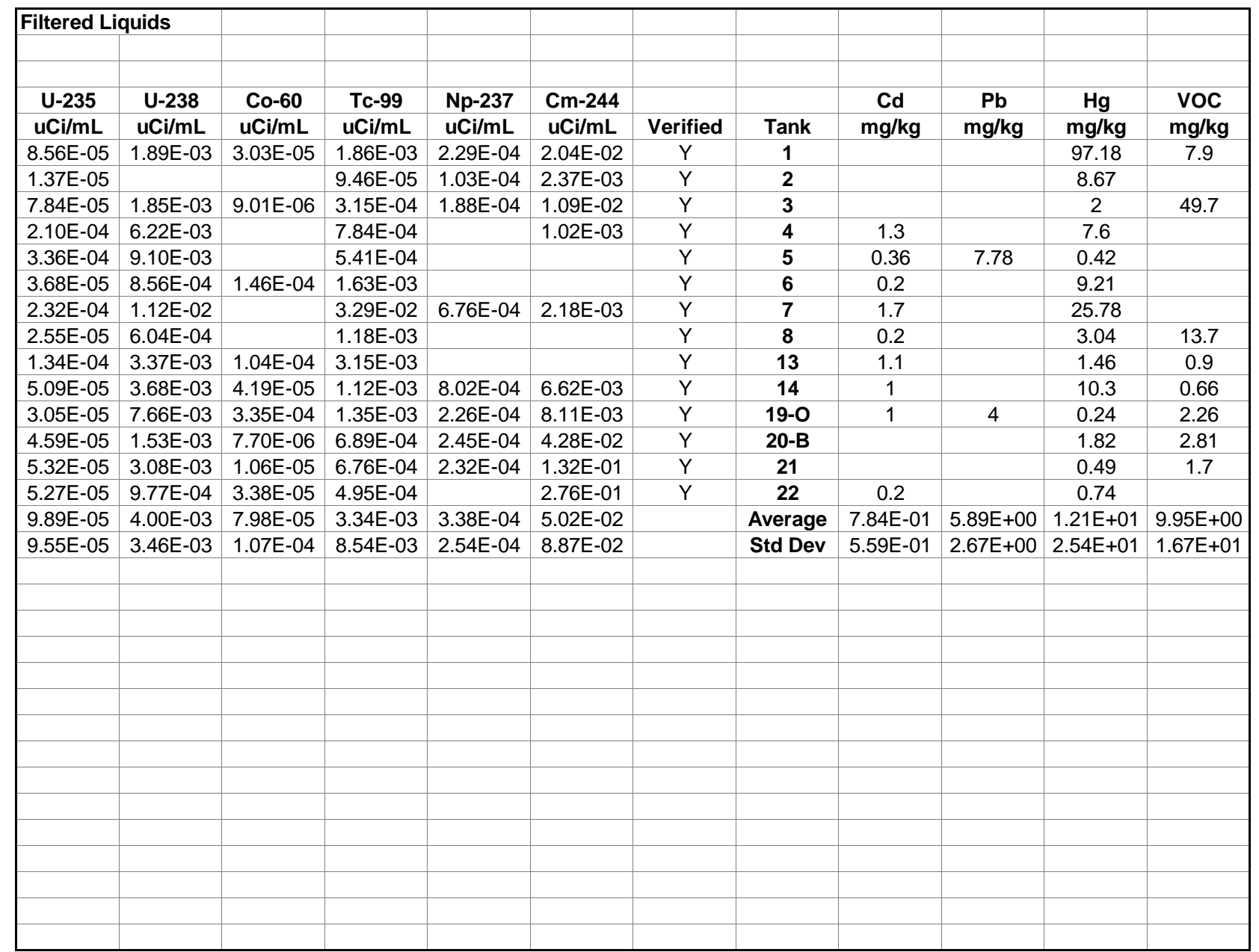




\begin{tabular}{|c|c|c|c|c|c|c|c|c|c|c|c|c|c|c|}
\hline \\
\hline \multicolumn{15}{|c|}{ Suspended Solids } \\
\hline & & $(\mathrm{uCi} / \mathrm{g})$ & (uCi/g) & (uCi/g) & (uCi/g) & $(\mathrm{uCi} / \mathrm{g})$ & $(\mathrm{uCi} / \mathrm{g})$ & (uCi/g) & $(\mathrm{uCi} / \mathrm{g})$ & (uCi/g) & (uCi/g) & (uCi/g) & (uCi/g) & (uCi/g) \\
\hline & Std Dev & 9.22E-01 & $2.87 \mathrm{E}+00$ & 1.91E-02 & $1.18 \mathrm{E}+01$ & $2.85 E+02$ & $1.70 \mathrm{E}+01$ & $1.13 E+02$ & $5.12 \mathrm{E}+01$ & 4.44E-03 & 8.46E-02 & $1.41 \mathrm{E}-02$ & 5.52E-02 & 2.68E-01 \\
\hline & Ave & 7.29E-01 & $3.06 \mathrm{E}+00$ & $1.28 \mathrm{E}-01$ & $6.22 \mathrm{E}+00$ & $1.61 \mathrm{E}+02$ & $9.95 \mathrm{E}+00$ & $5.07 \mathrm{E}+01$ & $4.51 \mathrm{E}+01$ & 3.89E-03 & 7.92E-02 & 2.13E-02 & 7.70E-02 & $1.08 \mathrm{E}-01$ \\
\hline Tank & Mass & H-3 & C-14 & I-129 & Cs-137 & Pu-238 & Pu-239 & Pu-241 & Sr-90 & $\mathrm{U}-235$ & $\mathrm{U}-238$ & Co-60 & Tc-99 & Np-237 \\
\hline$\#$ & grams & uCi & uCi & uCi & uCi & uCi & uCi & uCi & uCi & uCi & uCi & uCi & uCi & uCi \\
\hline 1 & $3.879 \mathrm{E}+01$ & $2.83 E+01$ & $1.19 \mathrm{E}+02$ & $4.95 \mathrm{E}+00$ & $2.41 \mathrm{E}+02$ & $6.23 \mathrm{E}+03$ & $3.86 \mathrm{E}+02$ & $1.97 \mathrm{E}+03$ & $1.75 \mathrm{E}+03$ & $1.51 \mathrm{E}-01$ & $3.07 \mathrm{E}+00$ & $8.25 \mathrm{E}-01$ & $2.99 \mathrm{E}+00$ & $4.21 \mathrm{E}+00$ \\
\hline 2 & $8.482 \mathrm{E}+01$ & $6.19 \mathrm{E}+01$ & $2.60 \mathrm{E}+02$ & $1.08 \mathrm{E}+01$ & $5.27 \mathrm{E}+02$ & $1.36 \mathrm{E}+04$ & $8.44 \mathrm{E}+02$ & $4.30 \mathrm{E}+03$ & $3.82 \mathrm{E}+03$ & $3.30 \mathrm{E}-01$ & $6.72 \mathrm{E}+00$ & $1.80 \mathrm{E}+00$ & $6.53 \mathrm{E}+00$ & $9.20 \mathrm{E}+00$ \\
\hline 3 & $2.197 \mathrm{E}+03$ & $1.60 \mathrm{E}+03$ & $6.72 \mathrm{E}+03$ & $2.80 \mathrm{E}+02$ & $1.37 \mathrm{E}+04$ & $3.53 \mathrm{E}+05$ & $2.19 \mathrm{E}+04$ & $1.11 \mathrm{E}+05$ & $9.90 \mathrm{E}+04$ & $8.55 \mathrm{E}+00$ & $1.74 \mathrm{E}+02$ & $4.67 \mathrm{E}+01$ & $1.69 \mathrm{E}+02$ & $2.38 \mathrm{E}+02$ \\
\hline 4 & $3.016 \mathrm{E}+02$ & $2.20 \mathrm{E}+02$ & $9.23 \mathrm{E}+02$ & $3.85 \mathrm{E}+01$ & $1.87 \mathrm{E}+03$ & $4.84 \mathrm{E}+04$ & $3.00 \mathrm{E}+03$ & $1.53 \mathrm{E}+04$ & $1.36 \mathrm{E}+04$ & $1.17 \mathrm{E}+00$ & $2.39 \mathrm{E}+01$ & $6.41 \mathrm{E}+00$ & $2.32 \mathrm{E}+01$ & $3.27 \mathrm{E}+01$ \\
\hline 5 & $3.480 \mathrm{E}+00$ & $2.54 \mathrm{E}+00$ & $1.07 \mathrm{E}+01$ & 4.44E-01 & $2.16 \mathrm{E}+01$ & $5.59 \mathrm{E}+02$ & $3.46 \mathrm{E}+01$ & $1.76 \mathrm{E}+02$ & $1.57 \mathrm{E}+02$ & 1.35E-02 & 2.76E-01 & 7.40E-02 & $2.68 \mathrm{E}-01$ & 3.77E-01 \\
\hline 6 & $1.650 \mathrm{E}+04$ & $1.20 \mathrm{E}+04$ & $5.05 \mathrm{E}+04$ & $2.10 \mathrm{E}+03$ & $1.03 \mathrm{E}+05$ & $2.65 E+06$ & $1.64 \mathrm{E}+05$ & $8.37 E+05$ & $7.44 \mathrm{E}+05$ & $6.43 \mathrm{E}+01$ & $1.31 \mathrm{E}+03$ & $3.51 \mathrm{E}+02$ & $1.27 \mathrm{E}+03$ & $1.79 \mathrm{E}+03$ \\
\hline 7 & $2.494 \mathrm{E}+03$ & $1.82 \mathrm{E}+03$ & $7.63 \mathrm{E}+03$ & $3.18 \mathrm{E}+02$ & $1.55 \mathrm{E}+04$ & $4.00 \mathrm{E}+05$ & $2.48 \mathrm{E}+04$ & $1.26 \mathrm{E}+05$ & $1.12 \mathrm{E}+05$ & $9.71 \mathrm{E}+00$ & $1.98 \mathrm{E}+02$ & $5.30 \mathrm{E}+01$ & $1.92 \mathrm{E}+02$ & $2.70 \mathrm{E}+02$ \\
\hline 8 & $4.906 \mathrm{E}+02$ & $3.58 \mathrm{E}+02$ & $1.50 \mathrm{E}+03$ & $6.25 \mathrm{E}+01$ & $3.05 \mathrm{E}+03$ & $7.88 \mathrm{E}+04$ & $4.88 \mathrm{E}+03$ & $2.49 \mathrm{E}+04$ & $2.21 \mathrm{E}+04$ & $1.91 \mathrm{E}+00$ & $3.89 \mathrm{E}+01$ & $1.04 \mathrm{E}+01$ & $3.78 \mathrm{E}+01$ & $5.32 \mathrm{E}+01$ \\
\hline 9 & $0.000 \mathrm{E}+00$ & $0.00 \mathrm{E}+00$ & $0.00 \mathrm{E}+00$ & $0.00 \mathrm{E}+00$ & $0.00 \mathrm{E}+00$ & $0.00 \mathrm{E}+00$ & $0.00 \mathrm{E}+00$ & $0.00 \mathrm{E}+00$ & $0.00 \mathrm{E}+00$ & $0.00 \mathrm{E}+00$ & $0.00 \mathrm{E}+00$ & $0.00 \mathrm{E}+00$ & $0.00 \mathrm{E}+00$ & $0.00 \mathrm{E}+00$ \\
\hline 10 & $8.433 \mathrm{E}+01$ & $6.15 \mathrm{E}+01$ & $2.58 \mathrm{E}+02$ & $1.08 \mathrm{E}+01$ & $5.24 \mathrm{E}+02$ & $1.35 \mathrm{E}+04$ & $8.39 \mathrm{E}+02$ & $4.28 \mathrm{E}+03$ & $3.80 \mathrm{E}+03$ & $3.28 \mathrm{E}-01$ & $6.68 \mathrm{E}+00$ & $1.79 \mathrm{E}+00$ & $6.49 \mathrm{E}+00$ & $9.14 \mathrm{E}+00$ \\
\hline 11 & $3.790 \mathrm{E}+00$ & $2.76 \mathrm{E}+00$ & $1.16 \mathrm{E}+01$ & 4.83E-01 & $2.36 \mathrm{E}+01$ & $6.08 \mathrm{E}+02$ & $3.77 \mathrm{E}+01$ & $1.92 \mathrm{E}+02$ & $1.71 \mathrm{E}+02$ & 1.48E-02 & $3.00 \mathrm{E}-01$ & 8.06E-02 & 2.92E-01 & $4.11 \mathrm{E}-01$ \\
\hline 12 & $4.217 \mathrm{E}+01$ & $3.08 \mathrm{E}+01$ & $1.29 \mathrm{E}+02$ & $5.38 \mathrm{E}+00$ & $2.62 \mathrm{E}+02$ & $6.77 \mathrm{E}+03$ & $4.20 \mathrm{E}+02$ & $2.14 \mathrm{E}+03$ & $1.90 \mathrm{E}+03$ & 1.64E-01 & $3.34 \mathrm{E}+00$ & 8.96E-01 & $3.25 \mathrm{E}+00$ & $4.57 \mathrm{E}+00$ \\
\hline 13 & $1.811 \mathrm{E}+02$ & $1.32 \mathrm{E}+02$ & $5.54 \mathrm{E}+02$ & $2.31 \mathrm{E}+01$ & $1.13 \mathrm{E}+03$ & $2.91 \mathrm{E}+04$ & $1.80 \mathrm{E}+03$ & $9.19 \mathrm{E}+03$ & $8.16 \mathrm{E}+03$ & 7.05E-01 & $1.43 \mathrm{E}+01$ & $3.85 \mathrm{E}+00$ & $1.39 \mathrm{E}+01$ & $1.96 \mathrm{E}+01$ \\
\hline 14 & $2.544 \mathrm{E}+02$ & $1.86 \mathrm{E}+02$ & $7.79 \mathrm{E}+02$ & $3.24 \mathrm{E}+01$ & $1.58 \mathrm{E}+03$ & $4.08 \mathrm{E}+04$ & $2.53 \mathrm{E}+03$ & $1.29 \mathrm{E}+04$ & $1.15 \mathrm{E}+04$ & $9.90 \mathrm{E}-01$ & $2.02 \mathrm{E}+01$ & $5.41 \mathrm{E}+00$ & $1.96 \mathrm{E}+01$ & $2.76 \mathrm{E}+01$ \\
\hline 15 & $0.000 \mathrm{E}+00$ & $0.00 \mathrm{E}+00$ & $0.00 \mathrm{E}+00$ & $0.00 \mathrm{E}+00$ & $0.00 \mathrm{E}+00$ & $0.00 \mathrm{E}+00$ & $0.00 \mathrm{E}+00$ & $0.00 \mathrm{E}+00$ & $0.00 \mathrm{E}+00$ & $0.00 \mathrm{E}+00$ & $0.00 \mathrm{E}+00$ & $0.00 \mathrm{E}+00$ & $0.00 \mathrm{E}+00$ & $0.00 \mathrm{E}+00$ \\
\hline 16 & $0.000 \mathrm{E}+00$ & $0.00 \mathrm{E}+00$ & $0.00 \mathrm{E}+00$ & $0.00 \mathrm{E}+00$ & $0.00 \mathrm{E}+00$ & $0.00 \mathrm{E}+00$ & $0.00 \mathrm{E}+00$ & $0.00 \mathrm{E}+00$ & $0.00 \mathrm{E}+00$ & $0.00 \mathrm{E}+00$ & $0.00 \mathrm{E}+00$ & $0.00 \mathrm{E}+00$ & $0.00 \mathrm{E}+00$ & $0.00 \mathrm{E}+00$ \\
\hline 17 & $0.000 \mathrm{E}+00$ & $0.00 \mathrm{E}+00$ & $0.00 \mathrm{E}+00$ & $0.00 \mathrm{E}+00$ & $0.00 \mathrm{E}+00$ & $0.00 \mathrm{E}+00$ & $0.00 \mathrm{E}+00$ & $0.00 \mathrm{E}+00$ & $0.00 \mathrm{E}+00$ & $0.00 \mathrm{E}+00$ & $0.00 \mathrm{E}+00$ & $0.00 \mathrm{E}+00$ & $0.00 \mathrm{E}+00$ & $0.00 \mathrm{E}+00$ \\
\hline 18 & $4.217 \mathrm{E}+01$ & $3.08 \mathrm{E}+01$ & $1.29 \mathrm{E}+02$ & $5.38 \mathrm{E}+00$ & $2.62 \mathrm{E}+02$ & $6.77 \mathrm{E}+03$ & $4.20 \mathrm{E}+02$ & $2.14 \mathrm{E}+03$ & $1.90 \mathrm{E}+03$ & 1.64E-01 & $3.34 \mathrm{E}+00$ & 8.96E-01 & $3.25 \mathrm{E}+00$ & $4.57 \mathrm{E}+00$ \\
\hline $19-0$ & $2.850 \mathrm{E}+03$ & $2.08 \mathrm{E}+03$ & $8.72 \mathrm{E}+03$ & $3.63 E+02$ & $1.77 \mathrm{E}+04$ & $4.57 \mathrm{E}+05$ & $2.84 \mathrm{E}+04$ & $1.44 \mathrm{E}+05$ & $1.28 \mathrm{E}+05$ & $1.11 \mathrm{E}+01$ & $2.26 \mathrm{E}+02$ & $6.06 \mathrm{E}+01$ & $2.19 \mathrm{E}+02$ & $3.09 \mathrm{E}+02$ \\
\hline 20-T & $1.112 \mathrm{E}+04$ & $8.11 \mathrm{E}+03$ & $3.40 \mathrm{E}+04$ & $1.42 \mathrm{E}+03$ & $6.91 \mathrm{E}+04$ & $1.79 \mathrm{E}+06$ & $1.11 \mathrm{E}+05$ & $5.64 \mathrm{E}+05$ & $5.01 \mathrm{E}+05$ & $4.33 \mathrm{E}+01$ & $8.81 \mathrm{E}+02$ & $2.36 \mathrm{E}+02$ & $8.56 \mathrm{E}+02$ & $1.21 \mathrm{E}+03$ \\
\hline 20-B & $1.587 \mathrm{E}+03$ & $1.16 \mathrm{E}+03$ & $4.86 \mathrm{E}+03$ & $2.02 \mathrm{E}+02$ & $9.87 \mathrm{E}+03$ & $2.55 \mathrm{E}+05$ & $1.58 \mathrm{E}+04$ & $8.05 \mathrm{E}+04$ & $7.15 \mathrm{E}+04$ & $6.18 \mathrm{E}+00$ & $1.26 \mathrm{E}+02$ & $3.37 \mathrm{E}+01$ & $1.22 \mathrm{E}+02$ & $1.72 \mathrm{E}+02$ \\
\hline 21 & $7.099 \mathrm{E}+03$ & $5.18 \mathrm{E}+03$ & $2.17 \mathrm{E}+04$ & $9.05 \mathrm{E}+02$ & $4.41 \mathrm{E}+04$ & $1.14 \mathrm{E}+06$ & $7.06 \mathrm{E}+04$ & $3.60 \mathrm{E}+05$ & $3.20 \mathrm{E}+05$ & $2.76 \mathrm{E}+01$ & $5.62 \mathrm{E}+02$ & $1.51 \mathrm{E}+02$ & $5.47 \mathrm{E}+02$ & $7.70 \mathrm{E}+02$ \\
\hline \multirow[t]{4}{*}{22} & $5.419 \mathrm{E}+02$ & $3.95 \mathrm{E}+02$ & $1.66 \mathrm{E}+03$ & $6.91 \mathrm{E}+01$ & $3.37 \mathrm{E}+03$ & $8.70 \mathrm{E}+04$ & $5.39 \mathrm{E}+03$ & $2.75 \mathrm{E}+04$ & $2.44 \mathrm{E}+04$ & $2.11 \mathrm{E}+00$ & $4.29 \mathrm{E}+01$ & $1.15 \mathrm{E}+01$ & 4.17E+01 & $5.87 \mathrm{E}+01$ \\
\hline & & & & & & & & & & & & & & \\
\hline & & & & & & & & & & & & & & \\
\hline & & & & & & & & & & & & & & \\
\hline \multicolumn{15}{|c|}{ C-14 Only UL's used for calculations, no MDA's used. No C-14 observed in samples. } \\
\hline \multicolumn{15}{|c|}{\begin{tabular}{|l|l|l} 
I-129 based upon UL's. MDA's not used. & & \\
\end{tabular}} \\
\hline \multicolumn{15}{|c|}{\begin{tabular}{|l|l|l} 
Tc-99 Only MDA's obtained,therefore they were used for calculations. & & \\
\end{tabular}} \\
\hline \multicolumn{15}{|c|}{\begin{tabular}{|l|l|l|} 
Others: detected activity only used to calculate average values, no MDA's, LL's, or UL's used. & & \\
\end{tabular}} \\
\hline \multicolumn{15}{|c|}{ Tanks 10,12, and 18 suspended solids masses calculated from average TSS values of aqueous tanks time volume in each respective tank. } \\
\hline \multicolumn{15}{|c|}{ Density of filtered liquids assumed to be $1.0 \mathrm{~g} / \mathrm{cm} 3$. } \\
\hline \multicolumn{15}{|c|}{ Ave. TSS = $0.223+/-0.438$ wt $\%$} \\
\hline \multicolumn{15}{|c|}{ No metals or VOC data taken on suspended solids. } \\
\hline \multirow{2}{*}{\multicolumn{15}{|c|}{$\begin{array}{l}\mathrm{Cd}, \mathrm{Pb} \text {, and } \mathrm{Hg} \text { based upon sludge data. } \\
\mathrm{VOC} \text { based unon filtered liquid data and }\end{array}$}} \\
\hline VOC base & on filtered lig & & & & & & & & & & & & & \\
\hline
\end{tabular}




\begin{tabular}{|c|c|c|c|c|c|c|c|c|c|c|c|c|c|c|}
\hline \multicolumn{2}{|c|}{ Suspended Solids } & & & & & \multicolumn{2}{|c|}{ Suspended Solids } & & & & & & & \\
\hline (uCi/g) & & $(\mathrm{mg} / \mathrm{kg})$ & $(\mathrm{mg} / \mathrm{kg})$ & $(\mathrm{mg} / \mathrm{kg})$ & $(\mathrm{mg} / \mathrm{kg})$ & & & & & & & & & \\
\hline $5.22 \mathrm{E}+02$ & Std Dev & $1.33 \mathrm{E}+03$ & $5.07 \mathrm{E}+04$ & $9.29 \mathrm{E}+03$ & $8.33 \mathrm{E}+00$ & & $\mathrm{H}-3$ & C-14 & I-129 & Cs-137 & Pu-238 & Pu-239 & Pu-241 & Sr-90 \\
\hline $2.62 \mathrm{E}+02$ & Ave & $9.14 \mathrm{E}+02$ & $2.99 \mathrm{E}+04$ & $1.23 \mathrm{E}+04$ & $4.98 \mathrm{E}+00$ & Tank & $u C i / g$ & $u C i / g$ & $u C i / g$ & $\mathbf{u C i} / \mathbf{g}$ & $\mathbf{u C i} / \mathbf{g}$ & $\mathrm{uCi} / \mathrm{g}$ & $\mathrm{uCi} / \mathrm{g}$ & $\mathrm{uCi} / \mathrm{g}$ \\
\hline $\mathrm{Cm}-244$ & Tank & Cd & $\mathbf{P b}$ & $\mathrm{Hg}$ & VOC & 1 & & & $9.46 E+00$ & & $9.32 \mathrm{E}+01$ & & $3.57 \mathrm{E}+01$ & $8.06 \mathrm{E}+01$ \\
\hline uCi & \# & mg & mg & mg & mg & 2 & & & & $6.53 \mathrm{E}-01$ & $1.34 \mathrm{E}+02$ & $4.73 \mathrm{E}+00$ & & $2.74 \mathrm{E}+00$ \\
\hline $1.01 \mathrm{E}+04$ & 1 & $3.55 \mathrm{E}+01$ & $1.16 \mathrm{E}+03$ & $4.76 \mathrm{E}+02$ & 1.93E-01 & 3 & $1.14 \mathrm{E}-01$ & & 7.21E-02 & $8.02 \mathrm{E}+00$ & $3.61 \mathrm{E}+01$ & $8.69 \mathrm{E}+00$ & $1.01 \mathrm{E}+01$ & $1.16 \mathrm{E}+02$ \\
\hline $2.22 \mathrm{E}+04$ & 2 & $7.75 \mathrm{E}+01$ & $2.54 \mathrm{E}+03$ & $1.04 \mathrm{E}+03$ & $4.22 \mathrm{E}-01$ & 4 & $1.28 \mathrm{E}+00$ & $5.09 \mathrm{E}+00$ & & $2.38 \mathrm{E}+00$ & $3.18 \mathrm{E}+01$ & $3.15 \mathrm{E}+00$ & $9.01 \mathrm{E}+00$ & \\
\hline $5.75 \mathrm{E}+05$ & 3 & $2.01 \mathrm{E}+03$ & $6.57 \mathrm{E}+04$ & $2.70 \mathrm{E}+04$ & $1.09 \mathrm{E}+01$ & 5 & & & & $1.71 \mathrm{E}+00$ & $1.29 \mathrm{E}+02$ & $6.89 \mathrm{E}+00$ & & \\
\hline $7.89 \mathrm{E}+04$ & 4 & $2.76 \mathrm{E}+02$ & $9.02 \mathrm{E}+03$ & $3.70 \mathrm{E}+03$ & $1.50 \mathrm{E}+00$ & 6 & $2.19 \mathrm{E}-02$ & 8.15E-02 & & 7.97E-01 & $3.24 \mathrm{E}+01$ & $5.50 \mathrm{E}+00$ & $5.77 \mathrm{E}+00$ & $3.31 \mathrm{E}+00$ \\
\hline $9.10 \mathrm{E}+02$ & 5 & $3.18 \mathrm{E}+00$ & $1.04 \mathrm{E}+02$ & $4.27 \mathrm{E}+01$ & 1.73E-02 & 7 & $1.58 \mathrm{E}-01$ & $2.03 \mathrm{E}+00$ & & 4.55E-01 & $3.50 \mathrm{E}+00$ & $5.45 \mathrm{E}+00$ & $1.23 \mathrm{E}+01$ & $7.39 \mathrm{E}+00$ \\
\hline $4.32 \mathrm{E}+06$ & 6 & $1.51 \mathrm{E}+04$ & $4.93 \mathrm{E}+05$ & $2.02 \mathrm{E}+05$ & $8.21 \mathrm{E}+01$ & 8 & $2.81 \mathrm{E}-01$ & 7.21E-01 & & $4.15 \mathrm{E}+01$ & $8.60 \mathrm{E}+01$ & $1.16 \mathrm{E}+01$ & $1.01 \mathrm{E}+01$ & $1.10 \mathrm{E}+02$ \\
\hline $6.52 \mathrm{E}+05$ & 7 & $2.28 \mathrm{E}+03$ & $7.46 \mathrm{E}+04$ & $3.06 \mathrm{E}+04$ & $1.24 \mathrm{E}+01$ & 13 & $2.68 \mathrm{E}+00$ & $7.70 \mathrm{E}+00$ & & $1.95 \mathrm{E}+01$ & $1.13 \mathrm{E}+03$ & $6.58 \mathrm{E}+01$ & $3.90 \mathrm{E}+02$ & $1.25 \mathrm{E}+02$ \\
\hline $1.28 \mathrm{E}+05$ & 8 & $4.49 \mathrm{E}+02$ & $1.47 \mathrm{E}+04$ & $6.02 \mathrm{E}+03$ & $2.44 \mathrm{E}+00$ & 14 & & & $2.21 E-02$ & 5.27E-01 & $2.48 \mathrm{E}+02$ & $1.57 \mathrm{E}+00$ & & \\
\hline $0.00 \mathrm{E}+00$ & 9 & $0.00 \mathrm{E}+00$ & $0.00 \mathrm{E}+00$ & $0.00 \mathrm{E}+00$ & $0.00 \mathrm{E}+00$ & $19-0$ & & & $2.98 E-02$ & 5.72E-01 & $9.19 \mathrm{E}+01$ & $2.21 \mathrm{E}+00$ & $2.86 \mathrm{E}+01$ & $1.73 \mathrm{E}+01$ \\
\hline $2.21 \mathrm{E}+04$ & 10 & $7.71 \mathrm{E}+01$ & $2.52 \mathrm{E}+03$ & $1.03 \mathrm{E}+03$ & 4.20E-01 & 20-B & & & $1.14 \mathrm{E}-01$ & $2.08 \mathrm{E}+00$ & $8.83 \mathrm{E}+01$ & $3.10 \mathrm{E}+00$ & $2.12 \mathrm{E}+01$ & $1.54 \mathrm{E}+01$ \\
\hline $9.91 \mathrm{E}+02$ & 11 & $3.47 \mathrm{E}+00$ & $1.13 \mathrm{E}+02$ & $4.65 \mathrm{E}+01$ & 1.89E-02 & 21 & 1.91E-01 & & $1.41 \mathrm{E}-01$ & $3.58 \mathrm{E}-01$ & $6.85 \mathrm{E}+01$ & $3.64 \mathrm{E}+00$ & $2.05 \mathrm{E}+01$ & $1.72 \mathrm{E}+01$ \\
\hline $1.10 \mathrm{E}+04$ & 12 & $3.86 \mathrm{E}+01$ & $1.26 \mathrm{E}+03$ & $5.17 \mathrm{E}+02$ & 2.10E-01 & 22 & $1.11 \mathrm{E}+00$ & $2.74 \mathrm{E}+00$ & & $2.25 \mathrm{E}+00$ & $7.48 \mathrm{E}+01$ & $7.03 \mathrm{E}+00$ & $1.45 \mathrm{E}+01$ & 6.85E-01 \\
\hline $4.74 \mathrm{E}+04$ & 13 & $1.66 \mathrm{E}+02$ & $5.42 \mathrm{E}+03$ & $2.22 \mathrm{E}+03$ & 9.02E-01 & Average & 7.29E-01 & $3.06 \mathrm{E}+00$ & $1.28 \mathrm{E}-01$ & $6.22 \mathrm{E}+00$ & $1.61 \mathrm{E}+02$ & $9.95 \mathrm{E}+00$ & $5.07 \mathrm{E}+01$ & $4.51 \mathrm{E}+01$ \\
\hline $6.65 \mathrm{E}+04$ & 14 & $2.33 \mathrm{E}+02$ & $7.61 \mathrm{E}+03$ & $3.12 \mathrm{E}+03$ & $1.27 \mathrm{E}+00$ & Std Dev & $9.22 \mathrm{E}-01$ & $2.87 \mathrm{E}+00$ & 1.91E-02 & $1.18 \mathrm{E}+01$ & $2.85 \mathrm{E}+02$ & $1.70 \mathrm{E}+01$ & $1.13 \mathrm{E}+02$ & $5.12 \mathrm{E}+01$ \\
\hline $0.00 \mathrm{E}+00$ & 15 & $0.00 \mathrm{E}+00$ & $0.00 \mathrm{E}+00$ & $0.00 \mathrm{E}+00$ & $0.00 \mathrm{E}+00$ & DL Ave & & & $4.13 \mathrm{E}-02$ & & & & & \\
\hline $0.00 \mathrm{E}+00$ & 16 & $0.00 \mathrm{E}+00$ & $0.00 \mathrm{E}+00$ & $0.00 \mathrm{E}+00$ & $0.00 \mathrm{E}+00$ & & & & \multicolumn{6}{|c|}{ I-129 average does not include the detection limit from Tank S1. } \\
\hline $0.00 \mathrm{E}+00$ & 17 & $0.00 \mathrm{E}+00$ & $0.00 \mathrm{E}+00$ & $0.00 \mathrm{E}+00$ & $0.00 \mathrm{E}+00$ & \multicolumn{4}{|c|}{ TSS Data for aqueous raw liquids } & \multicolumn{3}{|c|}{ Estimated Suspended Solids } & & \\
\hline $1.10 \mathrm{E}+04$ & 18 & $3.86 \mathrm{E}+01$ & $1.26 \mathrm{E}+03$ & $5.17 \mathrm{E}+02$ & 2.10E-01 & & & & & & & & & \\
\hline $7.45 \mathrm{E}+05$ & $19-0$ & $2.61 \mathrm{E}+03$ & $8.52 \mathrm{E}+04$ & $3.50 \mathrm{E}+04$ & $1.42 \mathrm{E}+01$ & Tank & Wt \% & & & & Volume & Est. Solids & & \\
\hline $2.91 \mathrm{E}+06$ & 20-T & $1.02 \mathrm{E}+04$ & $3.32 \mathrm{E}+05$ & $1.36 \mathrm{E}+05$ & $5.53 \mathrm{E}+01$ & 1 & 0.01 & & & Tank & $\mathbf{m L}$ & grams & & \\
\hline $4.15 \mathrm{E}+05$ & 20-B & $1.45 \mathrm{E}+03$ & $4.75 \mathrm{E}+04$ & $1.95 \mathrm{E}+04$ & $7.90 \mathrm{E}+00$ & 2 & 0.02 & & & 10 & 37854 & $8.43 \mathrm{E}+01$ & & \\
\hline $1.86 \mathrm{E}+06$ & 21 & $6.49 \mathrm{E}+03$ & $2.12 \mathrm{E}+05$ & $8.71 \mathrm{E}+04$ & $3.53 \mathrm{E}+01$ & 3 & 0.14 & & & 12 & 18927 & $4.22 \mathrm{E}+01$ & & \\
\hline \multirow[t]{15}{*}{$1.42 \mathrm{E}+05$} & 22 & $4.95 \mathrm{E}+02$ & $1.62 \mathrm{E}+04$ & $6.65 \mathrm{E}+03$ & $2.70 \mathrm{E}+00$ & 4 & 0.02 & & & 18 & 18927 & $4.22 \mathrm{E}+01$ & & \\
\hline & & & & & & 5 & 0.001 & & & & & & & \\
\hline & & & & & & 6 & 1.48 & & \multicolumn{4}{|c|}{ Calculation assumes density of liquids in tanks } & & \\
\hline & & & & & & 7 & 0.19 & & \multicolumn{3}{|c|}{10,12, and 18 are $1.0 \mathrm{~g} / \mathrm{cm} 3$} & & & \\
\hline & & & & & & 8 & 0.09 & & & & & & & \\
\hline & & & & & & 13 & 0.02 & & & & & & & \\
\hline & & & & & & 14 & 0.018 & & & & & & & \\
\hline & & & & & & $19-0$ & 0.12 & & & & & & & \\
\hline & & & & & & 20-B & 0.02 & & & & & & & \\
\hline & & & & & & 21 & 0.96 & & & & & & & \\
\hline & & & & & & 22 & 0.03 & & & & & & & \\
\hline & & & & & & Average & $2.23 \mathrm{E}-01$ & & & & & & & \\
\hline & & & & & & Std Dev & 4.38E-01 & & & & & & & \\
\hline & & & & & & & & & & & & & & \\
\hline & & & & & & & & & & & & & & \\
\hline
\end{tabular}




\begin{tabular}{|c|c|c|c|c|c|c|c|c|c|c|c|}
\hline \multicolumn{12}{|c|}{ Supsended Solids } \\
\hline U-235 & U-238 & Co-60 & Tc-99 & Np-237 & $\mathrm{Cm}-244$ & & & Cd & $\mathbf{P b}$ & $\mathrm{Hg}$ & VOC \\
\hline $\mathrm{uCi} / \mathrm{g}$ & $\mathrm{uCi} / \mathrm{g}$ & $\mathrm{uCi} / \mathrm{g}$ & $\mathrm{uCi} / \mathrm{g}$ & $\mathrm{uCi} / \mathrm{g}$ & $\mathrm{uCi} / \mathrm{g}$ & Verified & Tank & $\mathbf{m g} / \mathbf{k g}$ & $\mathrm{mg} / \mathrm{kg}$ & $\mathrm{mg} / \mathrm{kg}$ & $\mathrm{mg} / \mathrm{kg}$ \\
\hline $2.28 \mathrm{E}-03$ & $5.27 \mathrm{E}-02$ & & $1.61 \mathrm{E}-01$ & & $1.18 \mathrm{E}+02$ & $\mathrm{Y}$ & 1 & & & & \\
\hline $7.21 \mathrm{E}-03$ & $1.80 \mathrm{E}-01$ & & 5.32E-02 & & $2.54 \mathrm{E}+01$ & $Y$ & 2 & & & & \\
\hline 6.67E-03 & 1.36E-01 & & 2.95E-02 & $6.76 \mathrm{E}-02$ & $9.46 \mathrm{E}+01$ & $Y$ & 3 & & & & \\
\hline $4.21 \mathrm{E}-03$ & $8.60 \mathrm{E}-02$ & & & 4.03E-03 & 3.23E-01 & $\mathrm{Y}$ & 4 & & & & \\
\hline 9.95E-04 & 2.37E-02 & & & & & $\mathrm{Y}$ & 5 & & & & \\
\hline $6.71 \mathrm{E}-03$ & 2.22E-01 & $6.49 \mathrm{E}-03$ & & $9.14 \mathrm{E}-03$ & $1.09 \mathrm{E}+00$ & $\mathrm{Y}$ & 6 & & & & \\
\hline $4.01 \mathrm{E}-03$ & 1.45E-02 & & & 3.59E-03 & & $Y$ & 7 & & & & \\
\hline $2.48 \mathrm{E}-04$ & 5.41E-03 & $6.89 \mathrm{E}-03$ & & 1.61E-02 & 9.01E-01 & $Y$ & 8 & & & & \\
\hline 1.67E-02 & $2.55 E-01$ & 3.56E-02 & & $1.20 \mathrm{E}-01$ & & $\mathrm{Y}$ & 13 & & & & \\
\hline $1.80 \mathrm{E}-04$ & $4.48 \mathrm{E}-03$ & 3.38E-02 & & $3.41 \mathrm{E}-03$ & $1.36 \mathrm{E}+02$ & $\mathrm{Y}$ & 14 & & & & \\
\hline $1.90 \mathrm{E}-03$ & $4.59 \mathrm{E}-02$ & & 1.19E-01 & $9.10 \mathrm{E}-01$ & $1.18 \mathrm{E}+02$ & $\mathrm{Y}$ & $19-0$ & & & & \\
\hline $1.41 \mathrm{E}-03$ & 3.73E-02 & & $8.20 \mathrm{E}-02$ & $2.56 \mathrm{E}-02$ & $8.06 \mathrm{E}+01$ & $\mathrm{Y}$ & 20-B & & & & \\
\hline $1.32 \mathrm{E}-03$ & 3.21E-02 & & 1.72E-02 & 2.92E-02 & $5.32 \mathrm{E}+02$ & $\mathrm{Y}$ & 21 & & & & \\
\hline $6.62 \mathrm{E}-04$ & 1.39E-02 & $2.35 \mathrm{E}-02$ & & $3.99 \mathrm{E}-03$ & $1.77 \mathrm{E}+03$ & $\mathrm{Y}$ & 22 & & & & \\
\hline $3.89 \mathrm{E}-03$ & 7.92E-02 & $2.13 \mathrm{E}-02$ & 7.70E-02 & $1.08 \mathrm{E}-01$ & $2.62 \mathrm{E}+02$ & & Average & $\mathrm{N} / \mathrm{A}$ & $\mathrm{N} / \mathrm{A}$ & $\mathrm{N} / \mathrm{A}$ & $\mathrm{N} / \mathrm{A}$ \\
\hline 4.44E-03 & 8.46E-02 & $1.41 \mathrm{E}-02$ & 5.52E-02 & $2.68 \mathrm{E}-01$ & $5.22 \mathrm{E}+02$ & & Std Dev & N/A & $\mathrm{N} / \mathrm{A}$ & $\mathrm{N} / \mathrm{A}$ & $\mathrm{N} / \mathrm{A}$ \\
\hline & & & & & & & & & & & \\
\hline & & & & & & & & & & & \\
\hline & & & & & & & & & & & \\
\hline & & & & & & & & & & & \\
\hline & & & & & & & & & & & \\
\hline & & & & & & & & & & & \\
\hline & & & & & & & & & & & \\
\hline & & & & & & & & & & & \\
\hline & & & & & & & & & & & \\
\hline & & & & & & & & & & & \\
\hline & & & & & & & & & & & \\
\hline & & & & & & & & & & & \\
\hline & & & & & & & & & & & \\
\hline & & & & & & & & & & & \\
\hline & & & & & & & & & & & \\
\hline & & & & & & & & & & & \\
\hline & & & & & & & & & & & \\
\hline & & & & & & & & & & & \\
\hline & & & & & & & & & & & \\
\hline & & & & & & & & & & & \\
\hline & & & & & & & & & & & \\
\hline & & & & & & & & & & & \\
\hline & & & & & & & & & & & \\
\hline
\end{tabular}




\begin{tabular}{|c|c|c|c|c|c|c|c|c|c|c|c|c|c|c|c|c|}
\hline \multicolumn{17}{|c|}{ Solvent Tank Nuclide Inventory Calculations - Filtered Liquids } \\
\hline & $\mathrm{Ci} / \mathrm{g}$ & & $\mathrm{Ci} / \mathrm{g}$ & & $\mathrm{Ci} / \mathrm{g}$ & & $\mathrm{Ci} / \mathrm{g}$ & & $\mathrm{Ci} / \mathrm{g}$ & & $\mathrm{Ci} / \mathrm{g}$ & & $\mathrm{Ci} / \mathrm{g}$ & & $\mathrm{Ci} / \mathrm{g}$ & \\
\hline \multirow[t]{2}{*}{ SPCAT } & 9.64E-03 & & 6.24E-03 & & 2.16E-06 & & 6.47E-05 & & 3.36E-07 & & 7.05E-04 & & $1.71 \mathrm{E}+01$ & & 6.21E-02 & \\
\hline & U-233 & Error & U-234 & Error & U-235 & Error & U-236 & Error & U-238 & Error & Np-237 & Error & Pu-238 & Error & Pu-239 & Error \\
\hline Tank \# & $\mathrm{pCi} / \mathrm{L}$ & pCi/L & $\mathrm{pCi} / \mathrm{L}$ & $\mathrm{pCi} / \mathrm{L}$ & $\mathrm{pCi} / \mathrm{L}$ & pCi/L & $\mathrm{pCi} / \mathrm{L}$ & pCi/L & $\mathrm{pCi} / \mathrm{L}$ & $\mathrm{pCi} / \mathrm{L}$ & $\mathrm{pCi} / \mathrm{L}$ & $\mathrm{pCi} / \mathrm{L}$ & $\mathrm{pCi} / \mathrm{L}$ & $\mathrm{pCi} / \mathrm{L}$ & $\mathrm{pCi} / \mathrm{L}$ & pCi/L \\
\hline S1 & $5.05 E+05$ & $1.01 \mathrm{E}+05$ & $5.50 \mathrm{E}+06$ & $1.10 \mathrm{E}+06$ & $8.56 \mathrm{E}+04$ & $1.71 \mathrm{E}+04$ & $4.38 \mathrm{E}+04$ & $8.77 \mathrm{E}+03$ & $1.89 \mathrm{E}+06$ & $3.77 E+05$ & $2.29 \mathrm{E}+05$ & $1.15 \mathrm{E}+05$ & $6.49 \mathrm{E}+08$ & $6.49 E+07$ & $1.39 \mathrm{E}+07$ & $6.94 \mathrm{E}+06$ \\
\hline $\mathrm{S} 2$ & $0.00 \mathrm{E}+00$ & $0.00 \mathrm{E}+00$ & $2.97 E+06$ & $1.78 E+06$ & $1.37 \mathrm{E}+04$ & $5.31 E+03$ & $9.44 E+04$ & $1.40 \mathrm{E}+05$ & $4.00 \mathrm{E}+06$ & $6.92 \mathrm{E}+06$ & $1.03 \mathrm{E}+05$ & $2.62 \mathrm{E}+04$ & $1.42 \mathrm{E}+07$ & $2.84 \mathrm{E}+06$ & $1.98 E+06$ & $0.00 E+00$ \\
\hline S3 & $2.24 \mathrm{E}+06$ & $4.48 \mathrm{E}+05$ & $2.04 \mathrm{E}+06$ & $4.07 \mathrm{E}+05$ & $7.84 \mathrm{E}+04$ & $1.57 \mathrm{E}+04$ & $3.93 E+04$ & $7.86 \mathrm{E}+03$ & $1.85 \mathrm{E}+06$ & $3.70 \mathrm{E}+05$ & $1.88 \mathrm{E}+05$ & $9.39 E+04$ & $1.00 \mathrm{E}+07$ & $2.01 E+06$ & $1.05 \mathrm{E}+07$ & $5.25 \mathrm{E}+06$ \\
\hline S4 & $7.12 E+06$ & $0.00 E+00$ & $4.59 E+06$ & $0.00 E+00$ & $2.10 \mathrm{E}+05$ & $4.20 \mathrm{E}+04$ & $9.41 \mathrm{E}+04$ & $1.88 \mathrm{E}+04$ & $6.22 \mathrm{E}+06$ & $1.77 \mathrm{E}+06$ & $2.40 E+05$ & $0.00 E+00$ & $1.66 \mathrm{E}+07$ & $6.63 \mathrm{E}+06$ & $4.55 E+07$ & $0.00 E+00$ \\
\hline S5 & $1.12 \mathrm{E}+07$ & $2.24 \mathrm{E}+06$ & $1.28 \mathrm{E}+07$ & $2.57 \mathrm{E}+06$ & $3.36 \mathrm{E}+05$ & $6.72 \mathrm{E}+04$ & $2.06 \mathrm{E}+05$ & $4.12 \mathrm{E}+04$ & $9.10 \mathrm{E}+06$ & $1.82 \mathrm{E}+06$ & $4.95 E+05$ & $0.00 E+00$ & $3.28 \mathrm{E}+09$ & $1.31 \mathrm{E}+09$ & $2.27 \mathrm{E}+08$ & $4.55 \mathrm{E}+07$ \\
\hline S6 & $7.12 E+06$ & $0.00 E+00$ & $4.59 E+06$ & $0.00 E+00$ & $3.68 \mathrm{E}+04$ & $7.37 \mathrm{E}+03$ & $4.77 E+04$ & $0.00 E+00$ & $8.56 \mathrm{E}+05$ & $1.71 \mathrm{E}+05$ & $2.25 E+05$ & $0.00 E+00$ & $1.24 \mathrm{E}+07$ & $3.72 \mathrm{E}+06$ & $4.55 E+07$ & $0.00 E+00$ \\
\hline S7 & $7.12 E+06$ & $0.00 E+00$ & $4.59 E+06$ & $0.00 E+00$ & $2.32 \mathrm{E}+05$ & $1.72 \mathrm{E}+05$ & $1.99 \mathrm{E}+05$ & $3.97 \mathrm{E}+04$ & $1.12 \mathrm{E}+07$ & $2.24 \mathrm{E}+06$ & $6.76 \mathrm{E}+05$ & $2.70 \mathrm{E}+05$ & $1.71 \mathrm{E}+08$ & $5.12 \mathrm{E}+07$ & $1.02 \mathrm{E}+08$ & $4.07 \mathrm{E}+07$ \\
\hline S8 & $7.12 E+06$ & $0.00 E+00$ & $4.59 E+06$ & $0.00 E+00$ & $2.55 \mathrm{E}+04$ & $5.11 \mathrm{E}+03$ & $4.77 E+04$ & $0.00 E+00$ & $6.04 \mathrm{E}+05$ & $1.21 \mathrm{E}+05$ & $5.18 E+05$ & $0.00 E+00$ & $2.64 \mathrm{E}+07$ & $7.92 \mathrm{E}+06$ & $4.55 E+07$ & $0.00 E+00$ \\
\hline S9 & $4.90 \mathrm{E}+06$ & $9.30 \mathrm{E}+06$ & $4.39 E+06$ & $7.54 \mathrm{E}+06$ & $9.89 \mathrm{E}+04$ & $1.91 \mathrm{E}+05$ & $9.44 \mathrm{E}+04$ & $1.40 \mathrm{E}+05$ & $4.00 \mathrm{E}+06$ & $6.92 \mathrm{E}+06$ & $3.38 \mathrm{E}+05$ & $5.08 \mathrm{E}+05$ & $5.97 \mathrm{E}+08$ & $1.88 \mathrm{E}+09$ & 4.91E+07 & $1.57 \mathrm{E}+08$ \\
\hline $\mathrm{S} 10$ & $4.90 \mathrm{E}+06$ & $9.30 \mathrm{E}+06$ & $4.39 \mathrm{E}+06$ & $7.54 \mathrm{E}+06$ & $9.89 \mathrm{E}+04$ & $1.91 \mathrm{E}+05$ & $9.44 \mathrm{E}+04$ & $1.40 \mathrm{E}+05$ & $4.00 \mathrm{E}+06$ & $6.92 \mathrm{E}+06$ & $3.38 E+05$ & $5.08 \mathrm{E}+05$ & $5.97 \mathrm{E}+08$ & $1.88 \mathrm{E}+09$ & 4.91E+07 & $1.57 \mathrm{E}+08$ \\
\hline $\mathrm{S} 11$ & $6.76 E+04$ & $0.00 E+00$ & $4.35 E+04$ & $8.70 E+03$ & $2.02 \mathrm{E}+03$ & $4.04 \mathrm{E}+02$ & $4.55 \mathrm{E}+02$ & $9.10 \mathrm{E}+01$ & $4.86 E+04$ & $0.00 E+00$ & $4.95 \mathrm{E}+03$ & $9.91 E+02$ & $2.52 \mathrm{E}+09$ & $5.05 E+08$ & $4.34 \mathrm{E}+07$ & $8.68 \mathrm{E}+06$ \\
\hline $\mathrm{S} 12$ & $4.90 \mathrm{E}+06$ & $9.30 \mathrm{E}+06$ & $4.39 E+06$ & $7.54 \mathrm{E}+06$ & $9.89 \mathrm{E}+04$ & $1.91 E+05$ & $9.44 \mathrm{E}+04$ & $1.40 \mathrm{E}+05$ & $4.00 \mathrm{E}+06$ & $6.92 \mathrm{E}+06$ & $3.38 \mathrm{E}+05$ & $5.08 \mathrm{E}+05$ & $5.97 \mathrm{E}+08$ & $1.88 \mathrm{E}+09$ & 4.91E+07 & $1.57 \mathrm{E}+08$ \\
\hline $\mathrm{S} 13$ & $8.60 \mathrm{E}+06$ & $1.72 \mathrm{E}+06$ & $5.77 \mathrm{E}+06$ & $1.15 \mathrm{E}+06$ & $1.34 \mathrm{E}+05$ & $2.68 \mathrm{E}+04$ & $1.72 E+05$ & $3.43 E+04$ & $3.37 \mathrm{E}+06$ & $6.75 \mathrm{E}+05$ & $4.91 E+05$ & $0.00 E+00$ & $7.52 \mathrm{E}+08$ & $1.50 \mathrm{E}+08$ & $4.55 E+07$ & $0.00 E+00$ \\
\hline S14 & $3.29 E+05$ & $6.58 \mathrm{E}+04$ & $2.17 \mathrm{E}+06$ & $4.34 \mathrm{E}+05$ & $5.09 \mathrm{E}+04$ & $1.02 \mathrm{E}+04$ & $5.95 \mathrm{E}+04$ & $1.19 \mathrm{E}+04$ & $3.68 \mathrm{E}+06$ & $7.62 \mathrm{E}+05$ & $8.02 \mathrm{E}+05$ & $4.01 E+05$ & $1.08 \mathrm{E}+08$ & $2.16 \mathrm{E}+07$ & $8.74 \mathrm{E}+06$ & $4.37 \mathrm{E}+06$ \\
\hline S15 & $4.90 \mathrm{E}+06$ & $9.30 \mathrm{E}+06$ & $4.39 \mathrm{E}+06$ & $7.54 \mathrm{E}+06$ & $9.89 \mathrm{E}+04$ & $1.91 \mathrm{E}+05$ & $9.44 \mathrm{E}+04$ & $1.40 \mathrm{E}+05$ & $4.00 \mathrm{E}+06$ & $6.92 \mathrm{E}+06$ & $3.38 \mathrm{E}+05$ & $5.08 \mathrm{E}+05$ & $5.97 E+08$ & $1.88 E+09$ & $4.91 E+07$ & $1.57 E+08$ \\
\hline S16 & $4.90 \mathrm{E}+06$ & $9.30 \mathrm{E}+06$ & $4.39 \mathrm{E}+06$ & $7.54 \mathrm{E}+06$ & $9.89 \mathrm{E}+04$ & $1.91 \mathrm{E}+05$ & $9.44 \mathrm{E}+04$ & $1.40 \mathrm{E}+05$ & $4.00 \mathrm{E}+06$ & $6.92 \mathrm{E}+06$ & $3.38 \mathrm{E}+05$ & $5.08 \mathrm{E}+05$ & $5.97 E+08$ & $1.88 E+09$ & $4.91 E+07$ & $1.57 E+08$ \\
\hline S17 & $4.90 E+06$ & $9.30 \mathrm{E}+06$ & $4.39 \mathrm{E}+06$ & $7.54 \mathrm{E}+06$ & $9.89 \mathrm{E}+04$ & $1.91 \mathrm{E}+05$ & $9.44 \mathrm{E}+04$ & $1.40 \mathrm{E}+05$ & $4.00 \mathrm{E}+06$ & $6.92 \mathrm{E}+06$ & $3.38 \mathrm{E}+05$ & $5.08 \mathrm{E}+05$ & $5.97 E+08$ & $1.88 E+09$ & $4.91 E+07$ & $1.57 E+08$ \\
\hline S18 & $4.90 \mathrm{E}+06$ & $9.30 \mathrm{E}+06$ & $4.39 \mathrm{E}+06$ & $7.54 \mathrm{E}+06$ & $9.89 E+04$ & $1.91 \mathrm{E}+05$ & $9.44 \mathrm{E}+04$ & $1.40 \mathrm{E}+05$ & $4.00 \mathrm{E}+06$ & $6.92 \mathrm{E}+06$ & $3.38 \mathrm{E}+05$ & $5.08 \mathrm{E}+05$ & $5.97 E+08$ & $1.88 \mathrm{E}+09$ & $4.91 E+07$ & $1.57 E+08$ \\
\hline S19-O & $2.55 E+06$ & $5.10 \mathrm{E}+05$ & $1.59 \mathrm{E}+06$ & $3.19 \mathrm{E}+05$ & $3.05 E+04$ & $6.11 E+03$ & $3.08 \mathrm{E}+04$ & $6.16 \mathrm{E}+03$ & $7.66 \mathrm{E}+06$ & $1.20 \mathrm{E}+06$ & $2.26 \mathrm{E}+05$ & $1.13 E+05$ & $2.02 \mathrm{E}+09$ & $2.02 E+08$ & $5.95 E+06$ & $2.97 \mathrm{E}+06$ \\
\hline S19-A & $2.33 E+06$ & $4.66 \mathrm{E}+05$ & $1.64 \mathrm{E}+06$ & $3.28 \mathrm{E}+05$ & $3.08 \mathrm{E}+04$ & $6.15 \mathrm{E}+03$ & $2.97 E+04$ & $5.94 \mathrm{E}+03$ & $6.26 \mathrm{E}+06$ & $1.05 E+06$ & $2.49 \mathrm{E}+05$ & $1.24 \mathrm{E}+05$ & $2.03 E+09$ & $2.03 E+08$ & $4.77 E+06$ & $2.39 \mathrm{E}+06$ \\
\hline S20-T & $1.62 \mathrm{E}+05$ & $3.24 \mathrm{E}+04$ & 1.13E+05 & $2.25 \mathrm{E}+04$ & $2.11 E+03$ & $4.22 \mathrm{E}+02$ & $2.15 E+03$ & $4.31 \mathrm{E}+02$ & $9.23 \mathrm{E}+03$ & $1.85 E+03$ & $1.20 \mathrm{E}+04$ & $5.99 \mathrm{E}+03$ & $1.41 \mathrm{E}+06$ & $7.50 \mathrm{E}+05$ & $3.10 \mathrm{E}+05$ & $1.55 \mathrm{E}+05$ \\
\hline S20-B & $1.12 \mathrm{E}+07$ & $2.24 \mathrm{E}+06$ & $3.26 \mathrm{E}+06$ & $6.51 \mathrm{E}+05$ & $4.59 E+04$ & $9.19 E+03$ & $5.32 \mathrm{E}+04$ & $1.06 \mathrm{E}+04$ & $1.53 \mathrm{E}+06$ & $3.05 E+05$ & $2.45 \mathrm{E}+05$ & $1.22 \mathrm{E}+05$ & $5.59 \mathrm{E}+08$ & $5.59 \mathrm{E}+07$ & $1.21 \mathrm{E}+07$ & $6.06 \mathrm{E}+06$ \\
\hline S21 & $2.60 \mathrm{E}+06$ & $5.21 \mathrm{E}+05$ & $1.95 \mathrm{E}+06$ & $3.90 \mathrm{E}+05$ & $5.32 \mathrm{E}+04$ & $1.06 \mathrm{E}+04$ & $4.59 E+04$ & $9.19 \mathrm{E}+03$ & $3.08 \mathrm{E}+06$ & $6.58 \mathrm{E}+05$ & $2.32 \mathrm{E}+05$ & $1.16 \mathrm{E}+05$ & $4.68 \mathrm{E}+08$ & $1.41 \mathrm{E}+08$ & $1.26 \mathrm{E}+07$ & $6.28 \mathrm{E}+06$ \\
\hline S22 & $7.12 E+06$ & $0.00 E+00$ & $4.59 E+06$ & $0.00 E+00$ & $5.27 E+04$ & $1.05 E+04$ & $4.77 E+04$ & $0.00 E+00$ & 9.77E+05 & $1.95 \mathrm{E}+05$ & $2.07 E+05$ & $0.00 E+00$ & $2.76 \mathrm{E}+08$ & $5.51 \mathrm{E}+07$ & $4.55 E+07$ & $0.00 E+00$ \\
\hline \multicolumn{17}{|c|}{ Values listed in Black Bold Italics print are detection limits or upper limits. } \\
\hline \multicolumn{17}{|c|}{ Tank S2 U-233 included in U-234 activity, Pu-240 included in Pu-239 activity. U-236, U-238, Pu-242, Am-243, Cm-245, Cm-246 not measured } \\
\hline \multicolumn{17}{|c|}{\begin{tabular}{|l|l|l|l|l|} 
Tank S5 Cm-244 upper limit value estimated from Gross Alpha activity. & & & \\
\end{tabular}} \\
\hline \multicolumn{17}{|c|}{ The values calculated for samples S20-T and S20-B are upper limits. } \\
\hline \multicolumn{17}{|c|}{ Values listed in Blue Bold Italics are calculated based upon average activity concentrations of the analyzed aqueous solutions. } \\
\hline \multicolumn{17}{|c|}{\begin{tabular}{|l|l|} 
The organic liquids in Tank S11 and S20 (20-T) were not used to calculate average activity concentrations. & \\
\end{tabular}} \\
\hline \multicolumn{17}{|c|}{ Since S19-O and S19-A represent duplicate samples, only S19-O was used in the average activity calculations. } \\
\hline \multicolumn{17}{|c|}{\begin{tabular}{l|l|l} 
Average Pu-242, Cm-245, and $\mathrm{Cm}-246$ values calculated from detection limit numbers. & \\
\end{tabular}} \\
\hline Errors a & ssociated wi & th average a & ctivities were & e calculated & by averagin & th $\quad$ of & 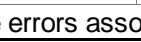 & & & & & & & & & \\
\hline
\end{tabular}




\begin{tabular}{|c|c|c|c|c|c|c|c|c|c|c|c|c|c|c|c|}
\hline $\mathrm{Ci} / \mathrm{g}$ & & $\mathrm{Ci} / \mathrm{g}$ & & $\mathrm{Ci} / \mathrm{g}$ & & $\mathrm{Ci} / \mathrm{g}$ & & $\mathrm{Ci} / \mathrm{g}$ & & $\mathrm{Ci} / \mathrm{g}$ & & $\mathrm{Ci} / \mathrm{g}$ & & $\mathrm{Ci} / \mathrm{g}$ & \\
\hline 2.27E-01 & & $1.03 E+02$ & & 3.93E-03 & & $3.44 \mathrm{E}+00$ & & 2.00E-01 & & $8.10 \mathrm{E}+01$ & & 1.72E-01 & & 3.04E-01 & \\
\hline Pu-240 & Error & Pu-241 & Error & Pu-242 & Error & Am-241 & Error & Am-243 & Error & Cm-244 & Error & $\mathrm{Cm}-245$ & Error & $\mathrm{Cm}-246$ & Error \\
\hline $\mathrm{pCi} / \mathrm{L}$ & $\mathrm{pCi} / \mathrm{L}$ & $\mathrm{pCi} / \mathrm{L}$ & pCi/L & $\mathrm{pCi} / \mathrm{L}$ & $\mathrm{pCi} / \mathrm{L}$ & pCi/L & pCi/L & pCi/L & pCi/L & $\mathrm{pCi} / \mathrm{L}$ & pCi/L & pCi/L & pCi/L & $\mathrm{pCi} / \mathrm{L}$ & pCi/L \\
\hline $1.97 \mathrm{E}+06$ & $9.86 \mathrm{E}+05$ & $1.60 \mathrm{E}+08$ & $4.81 \mathrm{E}+07$ & $1.81 E+04$ & $0.00 E+00$ & $8.42 \mathrm{E}+06$ & $4.36 \mathrm{E}+05$ & $9.95 E+04$ & $0.00 E+00$ & $2.04 \mathrm{E}+07$ & $4.07 \mathrm{E}+07$ & $7.88 E+05$ & $0.00 E+00$ & $1.41 E+06$ & $0.00 E+00$ \\
\hline $0.00 \mathrm{E}+00$ & $0.00 \mathrm{E}+00$ & $5.72 \mathrm{E}+06$ & $2.52 \mathrm{E}+06$ & $1.56 \mathrm{E}+06$ & $0.00 E+00$ & $3.14 \mathrm{E}+05$ & $2.29 \mathrm{E}+04$ & $1.31 \mathrm{E}+07$ & $0.00 \mathrm{E}+00$ & $2.37 \mathrm{E}+06$ & $4.75 \mathrm{E}+05$ & $6.82 E+07$ & $0.00 \mathrm{E}+00$ & $1.21 E+08$ & $0.00 \mathrm{E}+00$ \\
\hline $1.05 E+06$ & $0.00 E+00$ & $1.72 \mathrm{E}+07$ & 1.37E+07 & $1.81 E+04$ & $0.00 E+00$ & $1.15 E+05$ & $0.00 E+00$ & $3.61 E+04$ & $0.00 E+00$ & $1.09 \mathrm{E}+07$ & $1.53 \mathrm{E}+06$ & $7.88 E+05$ & $0.00 E+00$ & $1.41 E+06$ & $0.00 E+00$ \\
\hline $1.67 E+08$ & $0.00 E+00$ & $1.14 E+07$ & $0.00 E+00$ & $2.90 E+06$ & $0.00 E+00$ & $3.50 E+05$ & $0.00 E+00$ & $1.14 E+05$ & $0.00 E+00$ & $1.02 E+06$ & $2.05 E+06$ & $1.27 E+08$ & $0.00 E+00$ & $2.25 E+08$ & $0.00 E+00$ \\
\hline $2.24 \mathrm{E}+08$ & $4.49 \mathrm{E}+07$ & $8.11 \mathrm{E}+08$ & $3.24 \mathrm{E}+08$ & $2.75 E+06$ & $0.00 E+00$ & $1.39 \mathrm{E}+08$ & $5.60 \mathrm{E}+07$ & $1.40 E+08$ & $0.00 E+00$ & $2.49 E+11$ & $0.00 E+00$ & $1.20 E+08$ & $0.00 E+00$ & $2.14 E+08$ & $0.00 E+00$ \\
\hline $1.67 E+08$ & $0.00 E+00$ & $2.78 \mathrm{E}+07$ & 1.67E+07 & $2.90 E+06$ & $0.00 E+00$ & $3.14 E+05$ & $0.00 E+00$ & $1.05 E+05$ & $0.00 E+00$ & $6.17 E+05$ & $0.00 E+00$ & $1.27 E+08$ & $0.00 E+00$ & $2.25 E+08$ & $0.00 E+00$ \\
\hline $1.67 E+08$ & $0.00 E+00$ & $3.34 \mathrm{E}+08$ & $1.54 \mathrm{E}+08$ & $2.90 E+06$ & $0.00 E+00$ & $3.83 E+07$ & $1.94 \mathrm{E}+06$ & $8.56 E+04$ & $0.00 E+00$ & $2.18 E+06$ & $0.00 E+00$ & $1.27 E+08$ & $0.00 E+00$ & $2.25 E+08$ & $0.00 E+00$ \\
\hline $1.67 E+08$ & $0.00 E+00$ & $5.00 \mathrm{E}+08$ & $7.80 \mathrm{E}+08$ & $2.90 E+06$ & $0.00 E+00$ & $5.36 E+06$ & $0.00 E+00$ & $1.75 E+06$ & $0.00 E+00$ & $2.25 E+06$ & $0.00 E+00$ & $1.27 E+08$ & $0.00 E+00$ & $2.25 E+08$ & $0.00 E+00$ \\
\hline 4.02E+07 & $1.80 \mathrm{E}+08$ & $2.24 E+08$ & $4.96 \mathrm{E}+08$ & $1.56 \mathrm{E}+06$ & $0.00 \mathrm{E}+00$ & $2.11 E+07$ & $9.18 \mathrm{E}+07$ & $1.31 \mathrm{E}+07$ & $0.00 \mathrm{E}+00$ & $5.56 \mathrm{E}+07$ & $1.85 E+08$ & $6.82 \mathrm{E}+07$ & $0.00 \mathrm{E}+00$ & $1.21 E+08$ & $0.00 \mathrm{E}+00$ \\
\hline 4.02E+07 & $1.80 \mathrm{E}+08$ & $2.24 E+08$ & 4.96E+08 & $1.56 \mathrm{E}+06$ & $0.00 E+00$ & $2.11 E+07$ & $9.18 \mathrm{E}+07$ & $1.31 E+07$ & $0.00 E+00$ & $5.56 \mathrm{E}+07$ & $1.85 E+08$ & $6.82 \mathrm{E}+07$ & $0.00 E+00$ & $1.21 E+08$ & $0.00 E+00$ \\
\hline $1.59 \mathrm{E}+08$ & $3.18 \mathrm{E}+07$ & $1.14 \mathrm{E}+09$ & $4.54 \mathrm{E}+08$ & $2.75 E+06$ & $0.00 E+00$ & $3.71 E+06$ & $0.00 E+00$ & $1.45 E+06$ & $0.00 E+00$ & $5.09 E+09$ & $1.02 E+09$ & $1.20 E+08$ & $0.00 E+00$ & $2.14 \mathrm{E}+08$ & $4.28 \mathrm{E}+07$ \\
\hline 4.02E+07 & $1.80 E+08$ & $2.24 E+08$ & 4.96E+08 & $1.56 E+06$ & $0.00 E+00$ & $2.11 E+07$ & $9.18 \mathrm{E}+07$ & $1.31 E+07$ & $0.00 E+00$ & $5.56 \mathrm{E}+07$ & $1.85 E+08$ & $6.82 \mathrm{E}+07$ & $0.00 E+00$ & $1.21 E+08$ & $0.00 E+00$ \\
\hline $1.67 E+08$ & $0.00 E+00$ & $2.03 E+08$ & $8.13 \mathrm{E}+07$ & $2.90 E+06$ & $0.00 E+00$ & $7.52 E+05$ & $0.00 E+00$ & $2.32 E+05$ & $0.00 E+00$ & $2.26 E+07$ & $0.00 E+00$ & $1.27 E+08$ & $0.00 E+00$ & $2.25 E+08$ & $0.00 E+00$ \\
\hline 1.27E+06 & $6.35 \mathrm{E}+05$ & 2.39E+07 & $9.55 E+06$ & $1.81 E+04$ & $0.00 E+00$ & $1.41 \mathrm{E}+06$ & $1.64 \mathrm{E}+05$ & $8.33 E+04$ & $3.30 \mathrm{E}+04$ & $6.62 \mathrm{E}+06$ & $2.65 E+06$ & $7.88 E+05$ & $0.00 E+00$ & $1.41 E+06$ & $0.00 E+00$ \\
\hline 4.02E+07 & $1.80 \mathrm{E}+08$ & $2.24 E+08$ & $4.96 \mathrm{E}+08$ & $1.56 \mathrm{E}+06$ & $0.00 \mathrm{E}+00$ & $2.11 E+07$ & $9.18 \mathrm{E}+07$ & 1.31E+07 & $0.00 \mathrm{E}+00$ & $5.56 \mathrm{E}+07$ & $1.85 E+08$ & $6.82 \mathrm{E}+07$ & $0.00 \mathrm{E}+00$ & $1.21 E+08$ & $0.00 E+00$ \\
\hline $4.02 \mathrm{E}+07$ & $1.80 \mathrm{E}+08$ & $2.24 E+08$ & 4.96E+08 & $1.56 \mathrm{E}+06$ & $0.00 \mathrm{E}+00$ & $2.11 E+07$ & $9.18 \mathrm{E}+07$ & 1.31E+07 & $0.00 \mathrm{E}+00$ & $5.56 \mathrm{E}+07$ & $1.85 E+08$ & $6.82 \mathrm{E}+07$ & $0.00 \mathrm{E}+00$ & $1.21 E+08$ & $0.00 \mathrm{E}+00$ \\
\hline 4.02E+07 & $1.80 \mathrm{E}+08$ & $2.24 \mathrm{E}+08$ & $4.96 \mathrm{E}+08$ & $1.56 \mathrm{E}+06$ & $0.00 \mathrm{E}+00$ & $2.11 E+07$ & $9.18 \mathrm{E}+07$ & 1.31E+07 & $0.00 \mathrm{E}+00$ & $5.56 \mathrm{E}+07$ & $1.85 E+08$ & $6.82 \mathrm{E}+07$ & $0.00 \mathrm{E}+00$ & $1.21 E+08$ & $0.00 \mathrm{E}+00$ \\
\hline 4.02E+07 & $1.80 \mathrm{E}+08$ & $2.24 \mathrm{E}+08$ & 4.96E+08 & $1.56 \mathrm{E}+06$ & $0.00 E+00$ & $2.11 E+07$ & $9.18 \mathrm{E}+07$ & $1.31 E+07$ & $0.00 E+00$ & $5.56 \mathrm{E}+07$ & $1.85 E+08$ & $6.82 \mathrm{E}+07$ & $0.00 E+00$ & $1.21 E+08$ & $0.00 E+00$ \\
\hline $1.43 \mathrm{E}+06$ & 7.14E+05 & $5.18 \mathrm{E}+08$ & $1.55 \mathrm{E}+08$ & $1.81 E+04$ & $0.00 E+00$ & $9.05 E+05$ & $1.12 \mathrm{E}+05$ & $1.34 E+05$ & $0.00 E+00$ & $8.11 \mathrm{E}+06$ & $1.62 \mathrm{E}+07$ & $7.88 E+05$ & $0.00 E+00$ & $1.41 E+06$ & $0.00 E+00$ \\
\hline $1.57 \mathrm{E}+06$ & $7.84 \mathrm{E}+05$ & $5.23 \mathrm{E}+08$ & $1.57 \mathrm{E}+08$ & $1.81 E+04$ & $0.00 E+00$ & $9.05 E+05$ & $1.08 \mathrm{E}+05$ & $1.36 E+05$ & $0.00 E+00$ & $8.15 E+06$ & $1.63 \mathrm{E}+07$ & $7.88 E+05$ & $0.00 E+00$ & $1.41 E+06$ & $0.00 E+00$ \\
\hline $1.05 E+06$ & $0.00 E+00$ & $4.77 E+05$ & $0.00 E+00$ & $1.81 E+04$ & $0.00 E+00$ & $5.77 \mathrm{E}+05$ & $4.12 \mathrm{E}+04$ & $6.26 \mathrm{E}+04$ & $6.51 \mathrm{E}+03$ & 1.07E+07 & $1.73 \mathrm{E}+06$ & $7.88 E+05$ & $0.00 E+00$ & $1.41 E+06$ & $0.00 E+00$ \\
\hline $7.52 \mathrm{E}+06$ & $3.76 \mathrm{E}+06$ & $1.39 \mathrm{E}+08$ & $4.16 \mathrm{E}+07$ & $1.81 E+04$ & $0.00 E+00$ & $1.55 \mathrm{E}+05$ & $3.91 \mathrm{E}+04$ & $9.19 E+05$ & $0.00 E+00$ & $4.28 \mathrm{E}+07$ & $2.14 \mathrm{E}+07$ & $7.88 E+05$ & $0.00 E+00$ & $1.41 E+06$ & $0.00 E+00$ \\
\hline 4.73E+06 & $2.36 \mathrm{E}+06$ & $1.14 \mathrm{E}+08$ & $4.77 \mathrm{E}+07$ & $1.81 E+04$ & $0.00 E+00$ & $1.13 \mathrm{E}+06$ & $1.61 \mathrm{E}+05$ & $6.49 E+05$ & $0.00 E+00$ & $1.32 \mathrm{E}+08$ & $3.97 \mathrm{E}+07$ & $7.88 E+05$ & $0.00 E+00$ & $1.41 E+06$ & $0.00 E+00$ \\
\hline $1.67 E+08$ & $0.00 E+00$ & $6.44 \mathrm{E}+07$ & $2.58 \mathrm{E}+07$ & $2.90 E+06$ & $0.00 E+00$ & $6.53 E+05$ & $1.96 \mathrm{E}+05$ & $1.01 \mathrm{E}+06$ & $6.24 \mathrm{E}+04$ & $2.76 \mathrm{E}+08$ & $5.51 \mathrm{E}+07$ & $1.27 E+08$ & $0.00 E+00$ & $2.25 E+08$ & $0.00 E+00$ \\
\hline & & & & & & & & & & & & & & & \\
\hline & & & & & & & & & & & & & & & \\
\hline & & & & & & & & & & & & & & & \\
\hline & & & & & & & & & & & & & & & \\
\hline & & & & & & & & & & & & & & & \\
\hline & & & & & & & & & & & & & & & \\
\hline & & & & & & & & & & & & & & & \\
\hline & & & & & & & & & & & & & & & \\
\hline & & & & & & & & & & & & & & & \\
\hline
\end{tabular}




\begin{tabular}{|c|c|c|c|c|c|c|c|c|c|c|c|c|c|c|c|c|}
\hline \multicolumn{17}{|c|}{ Total Activity Including Error } \\
\hline & & & & & & & & & & & & & & & & \\
\hline & & & & & & & & & & & & & & & & \\
\hline & & & & & & & & & & & & & & & & \\
\hline Volume & U-233 & U-234 & U-235 & U-236 & U-238 & Np-237 & Pu-238 & Pu-239 & Pu-240 & Pu-241 & Pu-242 & Am-241 & Am-243 & $\mathrm{Cm}-244$ & $\mathrm{Cm}-245$ & $\mathrm{Cm}-246$ \\
\hline Liters & $\mathbf{C i}$ & $\mathbf{C i}$ & $\mathbf{C i}$ & $\mathbf{C i}$ & $\mathrm{Ci}$ & $\mathrm{Ci}$ & $\mathrm{Ci}$ & $\mathrm{Ci}$ & $\mathrm{Ci}$ & $\mathrm{Ci}$ & $\mathrm{Ci}$ & $\mathbf{C i}$ & $\mathrm{Ci}$ & $\mathrm{Ci}$ & $\mathbf{C i}$ & $\mathrm{Ci}$ \\
\hline 443.2717 & $2.69 \mathrm{E}-04$ & $2.93 \mathrm{E}-03$ & $4.55 \mathrm{E}-05$ & $2.33 \mathrm{E}-05$ & $1.00 \mathrm{E}-03$ & $1.52 \mathrm{E}-04$ & $3.16 \mathrm{E}-01$ & $9.24 \mathrm{E}-03$ & $1.31 \mathrm{E}-03$ & $9.22 \mathrm{E}-02$ & $8.02 E-06$ & $3.93 \mathrm{E}-03$ & $4.41 E-05$ & $2.71 \mathrm{E}-02$ & $3.49 E-04$ & $6.25 E-04$ \\
\hline 422.8305 & $0.00 \mathrm{E}+00$ & $2.01 E-03$ & 8.04E-06 & 9.91E-05 & 4.62E-03 & 5.46E-05 & $7.21 \mathrm{E}-03$ & 8.37E-04 & $0.00 \mathrm{E}+00$ & $3.48 \mathrm{E}-03$ & $6.60 \mathrm{E}-04$ & $1.42 \mathrm{E}-04$ & 5.54E-03 & $1.20 \mathrm{E}-03$ & 2.88E-02 & 5.12E-02 \\
\hline 1506.215 & 4.05E-03 & $3.69 E-03$ & 1.42E-04 & 7.10E-05 & 3.34E-03 & $4.25 \mathrm{E}-04$ & 1.81E-02 & 2.37E-02 & $1.58 E-03$ & 4.65E-02 & 2.73E-05 & $1.73 E-04$ & 5.44E-05 & 1.87E-02 & $1.19 E-03$ & $2.12 E-03$ \\
\hline 1397.574 & $9.95 E-03$ & $6.41 E-03$ & $3.52 \mathrm{E}-04$ & $1.58 \mathrm{E}-04$ & $1.12 \mathrm{E}-02$ & $3.35 E-04$ & $3.25 \mathrm{E}-02$ & $6.36 E-02$ & $2.33 E-01$ & $1.59 \mathrm{E}-02$ & $4.05 E-03$ & $4.89 E-04$ & $1.59 E-04$ & $4.29 E-03$ & $1.77 E-01$ & $3.14 E-01$ \\
\hline 348.2579 & $4.68 \mathrm{E}-03$ & 5.35E-03 & 1.40E-04 & 8.61E-05 & 3.80E-03 & 1.72E-04 & $1.60 \mathrm{E}+00$ & 9.49E-02 & 9.36E-02 & 3.95E-01 & $9.58 E-04$ & 6.79E-02 & $4.88 E-02$ & $8.67 E+01$ & $4.18 E-02$ & 7.45E-02 \\
\hline 1042.124 & 7.42E-03 & $4.78 E-03$ & 4.60E-05 & 4.97E-05 & 1.07E-03 & 2.34E-04 & 1.68E-02 & $4.74 E-02$ & $1.74 E-01$ & 4.64E-02 & $3.02 E-03$ & $3.27 E-04$ & $1.09 E-04$ & $6.43 E-04$ & $1.32 E-01$ & 2.34E-01 \\
\hline 1199.976 & $8.54 E-03$ & $5.51 E-03$ & $4.85 \mathrm{E}-04$ & 2.86E-04 & $1.61 \mathrm{E}-02$ & $1.14 \mathrm{E}-03$ & 2.67E-01 & $1.71 \mathrm{E}-01$ & $2.00 E-01$ & 5.86E-01 & $3.48 E-03$ & 4.83E-02 & $1.03 E-04$ & $2.62 E-03$ & $1.52 E-01$ & $2.70 E-01$ \\
\hline 518.6014 & $3.69 E-03$ & $2.38 E-03$ & 1.59E-05 & $2.47 E-05$ & $3.76 \mathrm{E}-04$ & $2.69 E-04$ & 1.78E-02 & $2.36 E-02$ & $8.66 E-02$ & 6.64E-01 & $1.50 E-03$ & $2.78 E-03$ & $9.08 E-04$ & 1.17E-03 & $6.59 E-02$ & 1.17E-01 \\
\hline 0 & $0.00 \mathrm{E}+00$ & $0.00 \mathrm{E}+00$ & $0.00 \mathrm{E}+00$ & $0.00 E+00$ & $0.00 E+00$ & $0.00 E+00$ & $0.00 \mathrm{E}+00$ & $0.00 E+00$ & $0.00 \mathrm{E}+00$ & $0.00 \mathrm{E}+00$ & $0.00 \mathrm{E}+00$ & $0.00 E+00$ & $0.00 E+00$ & $0.00 \mathrm{E}+00$ & $0.00 \mathrm{E}+00$ & $0.00 E+00$ \\
\hline 37.85412 & $5.38 \mathrm{E}-04$ & $4.52 \mathrm{E}-04$ & $1.10 \mathrm{E}-05$ & 8.87E-06 & 4.13E-04 & 3.20E-05 & $9.38 \mathrm{E}-02$ & $7.80 \mathrm{E}-03$ & 8.34E-03 & $2.73 \mathrm{E}-02$ & 5.91E-05 & 4.27E-03 & $4.96 \mathrm{E}-04$ & 9.11E-03 & $2.58 \mathrm{E}-03$ & $4.58 \mathrm{E}-03$ \\
\hline 54.13139 & $3.66 E-06$ & $2.83 E-06$ & $1.31 \mathrm{E}-07$ & $2.96 \mathrm{E}-08$ & $2.63 E-06$ & $3.22 \mathrm{E}-07$ & 1.64E-01 & $2.82 \mathrm{E}-03$ & $1.03 \mathrm{E}-02$ & 8.63E-02 & $1.49 E-04$ & $2.01 E-04$ & $7.85 E-05$ & $3.31 \mathrm{E}-01$ & $6.50 E-03$ & $1.39 \mathrm{E}-02$ \\
\hline 18.92706 & $2.69 \mathrm{E}-04$ & $2.26 \mathrm{E}-04$ & 5.49E-06 & 4.44E-06 & $2.07 \mathrm{E}-04$ & $1.60 \mathrm{E}-05$ & 4.69E-02 & $3.90 \mathrm{E}-03$ & 4.17E-03 & $1.36 \mathrm{E}-02$ & $2.95 \mathrm{E}-05$ & 2.14E-03 & $2.48 \mathrm{E}-04$ & $4.55 \mathrm{E}-03$ & $1.29 \mathrm{E}-03$ & $2.29 \mathrm{E}-03$ \\
\hline 826.3554 & 8.53E-03 & $5.72 \mathrm{E}-03$ & $1.33 \mathrm{E}-04$ & $1.70 \mathrm{E}-04$ & $3.34 \mathrm{E}-03$ & $4.06 E-04$ & $7.45 \mathrm{E}-01$ & $3.76 E-02$ & $1.38 E-01$ & $2.35 \mathrm{E}-01$ & $2.40 E-03$ & $6.21 E-04$ & $1.92 E-04$ & $1.87 E-02$ & $1.05 E-01$ & $1.86 E-01$ \\
\hline 1386.975 & $5.48 \mathrm{E}-04$ & $3.61 \mathrm{E}-03$ & $8.47 \mathrm{E}-05$ & $9.90 \mathrm{E}-05$ & $6.16 \mathrm{E}-03$ & $1.67 \mathrm{E}-03$ & 1.80E-01 & $1.82 \mathrm{E}-02$ & $2.64 \mathrm{E}-03$ & 4.64E-02 & $2.51 E-05$ & $2.18 \mathrm{E}-03$ & $1.61 \mathrm{E}-04$ & $1.29 \mathrm{E}-02$ & $1.09 E-03$ & $1.96 E-03$ \\
\hline 0 & $0.00 \mathrm{E}+00$ & $0.00 \mathrm{E}+00$ & $0.00 \mathrm{E}+00$ & $0.00 \mathrm{E}+00$ & $0.00 \mathrm{E}+00$ & $0.00 \mathrm{E}+00$ & $0.00 \mathrm{E}+00$ & $0.00 \mathrm{E}+00$ & $0.00 \mathrm{E}+00$ & $0.00 \mathrm{E}+00$ & $0.00 \mathrm{E}+00$ & $0.00 \mathrm{E}+00$ & $0.00 \mathrm{E}+00$ & $0.00 \mathrm{E}+00$ & $0.00 \mathrm{E}+00$ & $0.00 \mathrm{E}+00$ \\
\hline 0 & $0.00 \mathrm{E}+00$ & $0.00 \mathrm{E}+00$ & $0.00 \mathrm{E}+00$ & $0.00 \mathrm{E}+00$ & $0.00 \mathrm{E}+00$ & $0.00 \mathrm{E}+00$ & $0.00 \mathrm{E}+00$ & $0.00 \mathrm{E}+00$ & $0.00 \mathrm{E}+00$ & $0.00 \mathrm{E}+00$ & $0.00 \mathrm{E}+00$ & $0.00 \mathrm{E}+00$ & $0.00 \mathrm{E}+00$ & $0.00 \mathrm{E}+00$ & $0.00 \mathrm{E}+00$ & $0.00 \mathrm{E}+00$ \\
\hline 0 & $0.00 \mathrm{E}+00$ & $0.00 \mathrm{E}+00$ & $0.00 \mathrm{E}+00$ & $0.00 \mathrm{E}+00$ & $0.00 \mathrm{E}+00$ & $0.00 \mathrm{E}+00$ & $0.00 \mathrm{E}+00$ & $0.00 \mathrm{E}+00$ & $0.00 \mathrm{E}+00$ & $0.00 \mathrm{E}+00$ & $0.00 \mathrm{E}+00$ & $0.00 \mathrm{E}+00$ & $0.00 \mathrm{E}+00$ & $0.00 \mathrm{E}+00$ & $0.00 \mathrm{E}+00$ & $0.00 \mathrm{E}+00$ \\
\hline 18.92706 & $2.69 \mathrm{E}-04$ & $2.26 \mathrm{E}-04$ & $5.49 \mathrm{E}-06$ & 4.44E-06 & $2.07 \mathrm{E}-04$ & $1.60 \mathrm{E}-05$ & $4.69 \mathrm{E}-02$ & $3.90 \mathrm{E}-03$ & 4.17E-03 & $1.36 \mathrm{E}-02$ & $2.95 \mathrm{E}-05$ & $2.14 \mathrm{E}-03$ & $2.48 \mathrm{E}-04$ & $4.55 \mathrm{E}-03$ & $1.29 \mathrm{E}-03$ & $2.29 \mathrm{E}-03$ \\
\hline 2170.555 & $6.64 \mathrm{E}-03$ & $4.14 \mathrm{E}-03$ & $7.95 \mathrm{E}-05$ & $8.02 E-05$ & 1.92E-02 & 7.36E-04 & $4.82 \mathrm{E}+00$ & 1.94E-02 & $4.65 \mathrm{E}-03$ & $1.46 \mathrm{E}+00$ & $3.93 E-05$ & $2.21 \mathrm{E}-03$ & $2.91 E-04$ & $5.28 \mathrm{E}-02$ & $1.71 E-03$ & $3.06 E-03$ \\
\hline 2170.555 & $6.07 \mathrm{E}-03$ & $4.27 \mathrm{E}-03$ & $8.02 \mathrm{E}-05$ & 7.74E-05 & $1.59 \mathrm{E}-02$ & $8.10 \mathrm{E}-04$ & $4.85 E+00$ & $1.55 \mathrm{E}-02$ & $5.11 \mathrm{E}-03$ & $1.48 \mathrm{E}+00$ & 3.93E-05 & $2.20 \mathrm{E}-03$ & $2.95 E-04$ & 5.31E-02 & $1.71 E-03$ & $3.06 E-03$ \\
\hline 7551.897 & $1.47 \mathrm{E}-03$ & $1.02 \mathrm{E}-03$ & 1.91E-05 & $1.95 \mathrm{E}-05$ & $8.37 \mathrm{E}-05$ & 1.36E-04 & 1.63E-02 & $3.51 \mathrm{E}-03$ & 7.93E-03 & $3.60 E-03$ & $1.37 E-04$ & 4.67E-03 & $5.22 \mathrm{E}-04$ & $9.39 \mathrm{E}-02$ & $5.95 E-03$ & $1.06 E-02$ \\
\hline 7551.897 & $1.01 \mathrm{E}-01$ & $2.95 \mathrm{E}-02$ & $4.16 \mathrm{E}-04$ & $4.82 \mathrm{E}-04$ & $1.39 \mathrm{E}-02$ & $2.77 \mathrm{E}-03$ & $4.64 \mathrm{E}+00$ & $1.37 \mathrm{E}-01$ & 8.52E-02 & $1.36 \mathrm{E}+00$ & $1.37 E-04$ & $1.47 \mathrm{E}-03$ & $6.94 E-03$ & 4.85E-01 & $5.95 E-03$ & $1.06 E-02$ \\
\hline 690.4591 & $2.15 \mathrm{E}-03$ & $1.62 \mathrm{E}-03$ & 4.41E-05 & $3.80 \mathrm{E}-05$ & $2.58 \mathrm{E}-03$ & $2.40 \mathrm{E}-04$ & $4.20 \mathrm{E}-01$ & $1.30 \mathrm{E}-02$ & $4.90 \mathrm{E}-03$ & $1.12 \mathrm{E}-01$ & $1.25 E-05$ & 8.91E-04 & $4.48 E-04$ & 1.19E-01 & $5.44 E-04$ & $9.74 E-04$ \\
\hline 1694.35 & 1.21E-02 & $7.78 E-03$ & $1.07 \mathrm{E}-04$ & $8.08 E-05$ & 1.99E-03 & $3.51 E-04$ & 5.61E-01 & 7.71E-02 & $2.83 E-01$ & $1.53 \mathrm{E}-01$ & 4.91E-03 & $1.44 \mathrm{E}-03$ & $1.82 \mathrm{E}-03$ & 5.61E-01 & $2.15 E-01$ & $3.81 E-01$ \\
\hline & & & & & & & & & & & & & & & & \\
\hline & & & & & & & & & & & & & & & & \\
\hline & & & & & & & & & & & & & & & & \\
\hline & & & & & & & & & & & & & & & & \\
\hline & & & & & & & & & & & & & & & & \\
\hline & & & & & & & & & & & & & & & & \\
\hline & & & & & & & & & & & & & & & & \\
\hline & & & & & & & & & & & & & & & & \\
\hline & & & & & & & & & & & & & & & & \\
\hline & & & & & & & & & & & & & & & & \\
\hline
\end{tabular}




\begin{tabular}{|c|c|c|c|c|c|c|c|c|c|c|c|c|c|c|c|c|}
\hline \multicolumn{17}{|c|}{ Activity Concentration Including Error } \\
\hline & & & & & & & & & & & & & & & & \\
\hline & & & & & & & & & & & & & & & & \\
\hline & & & & & & & & & & & & & & & & \\
\hline & U-233 & U-234 & U-235 & U-236 & U-238 & Np-237 & Pu-238 & Pu-239 & Pu-240 & Pu-241 & Pu-242 & Am-241 & Am-243 & $\mathrm{Cm-244}$ & $\mathrm{Cm}-245$ & $\mathrm{Cm}-246$ \\
\hline Tank \# & $\mathrm{Ci} / \mathrm{L}$ & $\mathrm{Ci} / \mathrm{L}$ & $\mathrm{Ci} / \mathrm{L}$ & $\mathrm{Ci} / \mathrm{L}$ & $\mathrm{Ci} / \mathrm{L}$ & $\mathrm{Ci} / \mathrm{L}$ & $\mathrm{Ci} / \mathrm{L}$ & $\mathrm{Ci} / \mathrm{L}$ & $\mathrm{Ci} / \mathrm{L}$ & $\mathrm{Ci} / \mathrm{L}$ & $\mathrm{Ci} / \mathrm{L}$ & $\mathrm{Ci} / \mathrm{L}$ & $\mathrm{Ci} / \mathrm{L}$ & $\mathrm{Ci} / \mathrm{L}$ & $\mathrm{Ci} / \mathrm{L}$ & $\mathrm{Ci} / \mathrm{L}$ \\
\hline S1 & $6.06 \mathrm{E}-07$ & $6.60 \mathrm{E}-06$ & $1.03 \mathrm{E}-07$ & $5.26 \mathrm{E}-08$ & $2.27 \mathrm{E}-06$ & $3.44 \mathrm{E}-07$ & $7.14 \mathrm{E}-04$ & $2.08 \mathrm{E}-05$ & $2.96 \mathrm{E}-06$ & $2.08 \mathrm{E}-04$ & $1.81 E-08$ & $8.86 \mathrm{E}-06$ & $9.95 E-08$ & $6.11 \mathrm{E}-05$ & $7.88 E-07$ & 1.41E-06 \\
\hline S2 & $0.00 \mathrm{E}+00$ & $4.75 E-06$ & 1.90E-08 & 2.34E-07 & 1.09E-05 & 1.29E-07 & 1.70E-05 & $1.98 E-06$ & $0.00 \mathrm{E}+00$ & 8.24E-06 & 1.56E-06 & 3.37E-07 & 1.31E-05 & 2.85E-06 & 6.82E-05 & 1.21E-04 \\
\hline S3 & $2.69 E-06$ & $2.45 \mathrm{E}-06$ & $9.41 \mathrm{E}-08$ & 4.72E-08 & 2.22E-06 & 2.82E-07 & 1.20E-05 & 1.58E-05 & $1.05 E-06$ & 3.09E-05 & $1.81 E-08$ & 1.15E-07 & 3.61E-08 & 1.24E-05 & $7.88 E-07$ & 1.41E-06 \\
\hline S4 & 7.12E-06 & 4.59E-06 & 2.52E-07 & 1.13E-07 & 7.99E-06 & 2.40E-07 & 2.32E-05 & $4.55 E-05$ & $1.67 E-04$ & 1.14E-05 & 2.90E-06 & 3.50E-07 & $1.14 E-07$ & 3.07E-06 & $1.27 E-04$ & $2.25 E-04$ \\
\hline S5 & 1.34E-05 & 1.54E-05 & 4.03E-07 & 2.47E-07 & 1.09E-05 & $4.95 E-07$ & 4.59E-03 & 2.73E-04 & 2.69E-04 & 1.14E-03 & 2.75E-06 & 1.95E-04 & $1.40 E-04$ & 2.49E-01 & $1.20 E-04$ & 2.14E-04 \\
\hline S6 & 7.12E-06 & $4.59 E-06$ & 4.42E-08 & 4.77E-08 & 1.03E-06 & $2.25 E-07$ & 1.61E-05 & $4.55 E-05$ & $1.67 E-04$ & $4.45 \mathrm{E}-05$ & 2.90E-06 & 3.14E-07 & $1.05 E-07$ & 6.17E-07 & $1.27 E-04$ & $2.25 E-04$ \\
\hline S7 & 7.12E-06 & $4.59 E-06$ & 4.04E-07 & 2.39E-07 & 1.34E-05 & $9.46 \mathrm{E}-07$ & 2.22E-04 & 1.43E-04 & $1.67 E-04$ & $4.88 \mathrm{E}-04$ & 2.90E-06 & 4.02E-05 & $8.56 E-08$ & 2.18E-06 & $1.27 E-04$ & $2.25 E-04$ \\
\hline S8 & 7.12E-06 & $4.59 E-06$ & 3.06E-08 & 4.77E-08 & 7.25E-07 & $5.18 E-07$ & 3.43E-05 & 4.55E-05 & $1.67 E-04$ & $1.28 \mathrm{E}-03$ & 2.90E-06 & 5.36E-06 & $1.75 E-06$ & 2.25E-06 & $1.27 E-04$ & $2.25 E-04$ \\
\hline S9 & $0.00 \mathrm{E}+00$ & $0.00 \mathrm{E}+00$ & $0.00 \mathrm{E}+00$ & $0.00 \mathrm{E}+00$ & $0.00 \mathrm{E}+00$ & $0.00 \mathrm{E}+00$ & $0.00 \mathrm{E}+00$ & $0.00 \mathrm{E}+00$ & $0.00 \mathrm{E}+00$ & $0.00 \mathrm{E}+00$ & $0.00 \mathrm{E}+00$ & $0.00 \mathrm{E}+00$ & $0.00 \mathrm{E}+00$ & $0.00 \mathrm{E}+00$ & $0.00 \mathrm{E}+00$ & $0.00 \mathrm{E}+00$ \\
\hline S10 & 1.42E-05 & 1.19E-05 & 2.90E-07 & 2.34E-07 & 1.09E-05 & 8.46E-07 & 2.48E-03 & 2.06E-04 & 2.20E-04 & 7.20E-04 & 1.56E-06 & 1.13E-04 & 1.31E-05 & 2.41E-04 & 6.82E-05 & 1.21E-04 \\
\hline S11 & $6.76 E-08$ & $5.22 E-08$ & 2.42E-09 & $5.46 \mathrm{E}-10$ & $4.86 E-08$ & 5.94E-09 & 3.03E-03 & 5.21E-05 & 1.91E-04 & 1.59E-03 & $2.75 E-06$ & 3.71E-06 & $1.45 E-06$ & 6.11E-03 & $1.20 E-04$ & 2.57E-04 \\
\hline S12 & 1.42E-05 & 1.19E-05 & 2.90E-07 & 2.34E-07 & 1.09E-05 & 8.46E-07 & 2.48E-03 & 2.06E-04 & 2.20E-04 & 7.20E-04 & 1.56E-06 & 1.13E-04 & 1.31E-05 & 2.41E-04 & 6.82E-05 & 1.21E-04 \\
\hline $\mathrm{S} 13$ & 1.03E-05 & 6.92E-06 & $1.61 \mathrm{E}-07$ & 2.06E-07 & 4.05E-06 & $4.91 E-07$ & 9.02E-04 & $4.55 E-05$ & $1.67 E-04$ & 2.84E-04 & 2.90E-06 & 7.52E-07 & 2.32E-07 & 2.26E-05 & $1.27 E-04$ & $2.25 E-04$ \\
\hline S14 & $3.95 \mathrm{E}-07$ & $2.60 \mathrm{E}-06$ & $6.11 \mathrm{E}-08$ & 7.14E-08 & 4.44E-06 & 1.20E-06 & 1.30E-04 & $1.31 \mathrm{E}-05$ & $1.91 \mathrm{E}-06$ & 3.35E-05 & $1.81 E-08$ & 1.57E-06 & 1.16E-07 & 9.27E-06 & $7.88 E-07$ & $1.41 E-06$ \\
\hline S15 & $0.00 \mathrm{E}+00$ & $0.00 \mathrm{E}+00$ & $0.00 \mathrm{E}+00$ & $0.00 \mathrm{E}+00$ & $0.00 \mathrm{E}+00$ & $0.00 \mathrm{E}+00$ & $0.00 \mathrm{E}+00$ & $0.00 \mathrm{E}+00$ & $0.00 \mathrm{E}+00$ & $0.00 \mathrm{E}+00$ & $0.00 \mathrm{E}+00$ & $0.00 \mathrm{E}+00$ & $0.00 E+00$ & $0.00 \mathrm{E}+00$ & $0.00 \mathrm{E}+00$ & $0.00 \mathrm{E}+00$ \\
\hline S16 & $0.00 \mathrm{E}+00$ & $0.00 \mathrm{E}+00$ & $0.00 \mathrm{E}+00$ & $0.00 \mathrm{E}+00$ & $0.00 \mathrm{E}+00$ & $0.00 \mathrm{E}+00$ & $0.00 \mathrm{E}+00$ & $0.00 \mathrm{E}+00$ & $0.00 E+00$ & $0.00 \mathrm{E}+00$ & $0.00 E+00$ & $0.00 \mathrm{E}+00$ & $0.00 E+00$ & $0.00 \mathrm{E}+00$ & $0.00 \mathrm{E}+00$ & $0.00 \mathrm{E}+00$ \\
\hline S17 & $0.00 \mathrm{E}+00$ & $0.00 \mathrm{E}+00$ & $0.00 \mathrm{E}+00$ & $0.00 \mathrm{E}+00$ & $0.00 \mathrm{E}+00$ & $0.00 \mathrm{E}+00$ & $0.00 \mathrm{E}+00$ & $0.00 \mathrm{E}+00$ & $0.00 \mathrm{E}+00$ & $0.00 \mathrm{E}+00$ & $0.00 \mathrm{E}+00$ & $0.00 \mathrm{E}+00$ & $0.00 \mathrm{E}+00$ & $0.00 \mathrm{E}+00$ & $0.00 \mathrm{E}+00$ & $0.00 \mathrm{E}+00$ \\
\hline $\mathrm{S} 18$ & $1.42 E-05$ & 1.19E-05 & 2.90E-07 & 2.34E-07 & 1.09E-05 & 8.46E-07 & 2.48E-03 & $2.06 \mathrm{E}-04$ & $2.20 \mathrm{E}-04$ & $7.20 \mathrm{E}-04$ & $1.56 \mathrm{E}-06$ & 1.13E-04 & 1.31E-05 & 2.41E-04 & $6.82 \mathrm{E}-05$ & 1.21E-04 \\
\hline S19-O & 3.06E-06 & $1.91 \mathrm{E}-06$ & $3.66 \mathrm{E}-08$ & $3.70 \mathrm{E}-08$ & 8.86E-06 & 3.39E-07 & $2.22 \mathrm{E}-03$ & 8.92E-06 & $2.14 \mathrm{E}-06$ & 6.73E-04 & $1.81 E-08$ & 1.02E-06 & $1.34 E-07$ & 2.43E-05 & $7.88 E-07$ & 1.41E-06 \\
\hline S19-A & $2.80 \mathrm{E}-06$ & 1.97E-06 & $3.70 \mathrm{E}-08$ & 3.56E-08 & 7.31E-06 & 3.73E-07 & $2.23 \mathrm{E}-03$ & 7.16E-06 & $2.35 \mathrm{E}-06$ & $6.80 \mathrm{E}-04$ & $1.81 E-08$ & $1.01 \mathrm{E}-06$ & $1.36 E-07$ & 2.45E-05 & $7.88 E-07$ & 1.41E-06 \\
\hline S20-T & 1.94E-07 & 1.36E-07 & $2.53 E-09$ & $2.58 \mathrm{E}-09$ & $1.11 \mathrm{E}-08$ & $1.80 \mathrm{E}-08$ & $2.16 \mathrm{E}-06$ & 4.65E-07 & $1.05 E-06$ & 4.77E-07 & $1.81 E-08$ & 6.18E-07 & 6.91E-08 & $1.24 \mathrm{E}-05$ & $7.88 E-07$ & 1.41E-06 \\
\hline S20-B & 1.34E-05 & 3.91E-06 & $5.51 \mathrm{E}-08$ & $6.38 \mathrm{E}-08$ & $1.84 \mathrm{E}-06$ & 3.67E-07 & $6.15 \mathrm{E}-04$ & 1.82E-05 & $1.13 \mathrm{E}-05$ & 1.81E-04 & $1.81 E-08$ & 1.94E-07 & 9.19E-07 & 6.42E-05 & $7.88 E-07$ & 1.41E-06 \\
\hline $\mathrm{S} 21$ & 3.12E-06 & 2.34E-06 & 6.38E-08 & 5.51E-08 & 3.74E-06 & $3.48 \mathrm{E}-07$ & 6.09E-04 & 1.89E-05 & 7.09E-06 & 1.62E-04 & $1.81 E-08$ & $1.29 \mathrm{E}-06$ & 6.49E-07 & 1.72E-04 & $7.88 E-07$ & 1.41E-06 \\
\hline S22 & 7.12E-06 & 4.59E-06 & 6.32E-08 & 4.77E-08 & 1.17E-06 & 2.07E-07 & 3.31E-04 & $4.55 E-05$ & $1.67 E-04$ & 9.02E-05 & 2.90E-06 & 8.49E-07 & 1.07E-06 & 3.31E-04 & $1.27 E-04$ & $2.25 E-04$ \\
\hline & & & & & & & & & & & & & & & & \\
\hline & & & & & & & & & & & & & & & & \\
\hline & & & & & & & & & & & & & & & & \\
\hline & & & & & & & & & & & & & & & & \\
\hline & & & & & & & & & & & & & & & & \\
\hline & & & & & & & & & & & & & & & & \\
\hline & & & & & & & & & & & & & & & & \\
\hline & & & & & & & & & & & & & & & & \\
\hline & & & & & & & & & & & & & & & & \\
\hline & & & & & & & & & & & & & & & & \\
\hline
\end{tabular}




\begin{tabular}{|c|c|c|c|c|c|c|c|c|c|c|c|c|c|c|c|c|}
\hline & & & & & & & & & & & & & & & & \\
\hline & & & & & & & & & & & & & & & & \\
\hline & & & & & & & & & & & & & & & & \\
\hline & & & & & & & & & & & & & & & & \\
\hline & & & & & & & & & & & & & & & & \\
\hline Tank \# & $\begin{array}{c}U-233 \\
a\end{array}$ & U-234 & U-235 & U-236 & U-238 & Np-23r & $P u-238$ & Pu-239 & Pu-240 & Pu-241 & Pu-242 & Am-241 & Am-243 & $\mathrm{Cm}-244$ & $\mathrm{Cm}-245$ & $\mathrm{Cm}-246$ \\
\hline S1 & $\frac{\mathrm{g}}{279 \mathrm{E}-02}$ & $\frac{\mathbf{g}}{469 \mathrm{E}-01}$ & $\frac{\mathrm{g}}{211 \mathrm{~F}+01}$ & $\frac{9}{360 F-01}$ & $\frac{\mathrm{g}}{20050}$ & $\frac{\mathrm{g}}{21601}$ & $\frac{\mathrm{g}}{185 \mathrm{~F}_{0}}$ & $\frac{\mathrm{g}}{19 \mathrm{~F}_{-} 01}$ & $\frac{g}{577}$ & $\frac{9}{002501}$ & $\frac{9}{2002}$ & $\frac{\mathrm{g}}{114 \mathrm{R}}$ & $\frac{\mathrm{g}}{215}$ & $\frac{\mathrm{g}}{\mathrm{n}}$ & $\frac{\mathrm{g}}{202}$ & $\frac{g}{206 F-03}$ \\
\hline S2 & $0.00 \mathrm{~F}+000$ & 4.69E-01 & $2.11 \mathrm{E}+01$ & $3.60 \mathrm{E}-01$ & $2.99 \mathrm{E}+03$ & 2.16E-01 & 1.85E-02 & 1.49E-01 & 5.77E-03 & 8.92E-04 & $2.04 t-03$ & $1.14 \mathrm{E}-03$ & 2.21E-04 & 3.34E-04 & 2.03E-03 & 2.06E-03 \\
\hline & $0.00 \mathrm{E}+00$ & $3.22 E-01$ & $3.72 \mathrm{E}+00$ & $1.53 \mathrm{E}+00$ & 1.37E+04 & 7.75E-02 & 4.21E-04 & $1.35 E-02$ & $0.00 \mathrm{E}+00$ & 3.37E-05 & 1.68E-01 & 4.14E-05 & 2.77E-02 & 1.49E-05 & $1.68 \mathrm{E}-01$ & 1.68E-01 \\
\hline S3 & 4.20E-01 & 5.91E-01 & $6.56 \mathrm{E}+01$ & $1.10 \mathrm{E}+00$ & $9.95 \mathrm{E}+03$ & $6.02 \mathrm{E}-01$ & 1.06E-03 & 3.82E-01 & $6.97 E-03$ & $4.50 \mathrm{E}-04$ & $6.94 E-03$ & $5.04 E-05$ & $2.72 E-04$ & 2.31E-04 & $6.90 E-03$ & $6.99 E-03$ \\
\hline S4 & $1.03 E+00$ & $1.03 E+00$ & $1.63 \mathrm{E}+02$ & $2.44 \mathrm{E}+00$ & $3.32 \mathrm{E}+04$ & 4.76E-01 & 1.90E-03 & $1.02 E+00$ & $1.03 E+00$ & $1.54 E-04$ & $1.03 E+00$ & $1.42 E-04$ & 7.97E-04 & $5.30 E-05$ & $1.03 E+00$ & $1.03 E+00$ \\
\hline S5 & 4.86E-01 & $8.58 \mathrm{E}-01$ & $6.50 \mathrm{E}+01$ & $1.33 \mathrm{E}+00$ & $1.13 \mathrm{E}+04$ & $2.45 E-01$ & 9.35E-02 & $1.53 \mathrm{E}+00$ & 4.13E-01 & 3.82E-03 & $2.44 E-01$ & 1.97E-02 & $2.44 E-01$ & $1.07 E+00$ & $2.43 E-01$ & $2.45 E-01$ \\
\hline S6 & 7.70E-01 & 7.67E-01 & $2.13 \mathrm{E}+01$ & $7.68 E-01$ & $3.19 \mathrm{E}+03$ & 3.33E-01 & 9.82E-04 & $7.64 E-01$ & 7.67E-01 & $4.48 \mathrm{E}-04$ & $7.69 E-01$ & $9.51 E-05$ & 5.47E-04 & 7.94E-06 & $7.69 E-01$ & 7.71E-01 \\
\hline S7 & $8.86 E-01$ & $8.83 E-01$ & $2.24 \mathrm{E}+02$ & $4.43 \mathrm{E}+00$ & $4.80 \mathrm{E}+04$ & $1.61 \mathrm{E}+00$ & 1.56E-02 & $2.76 \mathrm{E}+00$ & $8.83 E-01$ & $5.66 \mathrm{E}-03$ & $8.85 E-01$ & $1.40 \mathrm{E}-02$ & $5.14 E-04$ & $3.23 E-05$ & $8.86 E-01$ & $8.88 E-01$ \\
\hline S8 & $3.83 E-01$ & $3.81 E-01$ & $7.35 \mathrm{E}+00$ & $3.82 E-01$ & $1.12 \mathrm{E}+03$ & $3.81 E-01$ & 1.04E-03 & $3.80 E-01$ & $3.82 E-01$ & 6.42E-03 & $3.83 E-01$ & 8.08E-04 & $4.54 E-03$ & $1.44 E-05$ & $3.83 E-01$ & $3.84 E-01$ \\
\hline S9 & $0.00 \mathrm{E}+00$ & $0.00 \mathrm{E}+00$ & $0.00 \mathrm{E}+00$ & $0.00 E+00$ & $0.00 \mathrm{E}+00$ & $0.00 \mathrm{E}+00$ & $0.00 \mathrm{E}+00$ & $0.00 \mathrm{E}+00$ & $0.00 \mathrm{E}+00$ & $0.00 \mathrm{E}+00$ & $0.00 \mathrm{E}+00$ & $0.00 \mathrm{E}+00$ & $0.00 E+00$ & $0.00 \mathrm{E}+00$ & $0.00 \mathrm{E}+00$ & $0.00 \mathrm{E}+00$ \\
\hline $\mathrm{S} 10$ & $5.58 \mathrm{E}-02$ & 7.24E-02 & $5.08 \mathrm{E}+00$ & 1.37E-01 & $1.23 E+03$ & 4.54E-02 & $5.48 \mathrm{E}-03$ & $1.26 \mathrm{E}-01$ & $3.67 \mathrm{E}-02$ & $2.64 \mathrm{E}-04$ & $1.50 \mathrm{E}-02$ & $1.24 \mathrm{E}-03$ & $2.48 \mathrm{E}-03$ & 1.12E-04 & $1.50 \mathrm{E}-02$ & $1.51 \mathrm{E}-02$ \\
\hline $\mathrm{S} 11$ & $3.80 E-04$ & $4.53 E-04$ & 6.07E-02 & 4.57E-04 & $7.83 E+00$ & 4.56E-04 & $9.58 \mathrm{E}-03$ & 4.54E-02 & 4.55E-02 & 8.34E-04 & $3.79 E-02$ & $5.84 E-05$ & 3.92E-04 & $4.08 \mathrm{E}-03$ & $3.78 E-02$ & $4.57 \mathrm{E}-02$ \\
\hline $\mathrm{S} 12$ & $2.79 \mathrm{E}-02$ & 3.62E-02 & $2.54 \mathrm{E}+00$ & 6.86E-02 & $6.15 \mathrm{E}+02$ & $2.27 \mathrm{E}-02$ & $2.74 \mathrm{E}-03$ & $6.28 \mathrm{E}-02$ & 1.84E-02 & $1.32 \mathrm{E}-04$ & 7.51E-03 & $6.21 \mathrm{E}-04$ & $1.24 \mathrm{E}-03$ & 5.62E-05 & $7.50 \mathrm{E}-03$ & $7.53 \mathrm{E}-03$ \\
\hline $\mathrm{S} 13$ & $8.85 \mathrm{E}-01$ & 9.16E-01 & $6.15 \mathrm{E}+01$ & $2.63 \mathrm{E}+00$ & $9.95 \mathrm{E}+03$ & $5.76 E-01$ & 4.36E-02 & $6.05 E-01$ & $6.08 E-01$ & 2.27E-03 & $6.10 E-01$ & $1.81 E-04$ & 9.59E-04 & $2.31 E-04$ & $6.10 E-01$ & $6.12 E-01$ \\
\hline S14 & 5.68E-02 & 5.79E-01 & $3.92 \mathrm{E}+01$ & $1.53 \mathrm{E}+00$ & $1.83 \mathrm{E}+04$ & $2.37 \mathrm{E}+00$ & $1.05 \mathrm{E}-02$ & 2.93E-01 & 1.16E-02 & 4.49E-04 & $6.39 E-03$ & $6.35 E-04$ & 8.07E-04 & 1.59E-04 & $6.35 E-03$ & $6.43 E-03$ \\
\hline $\mathrm{S} 15$ & $0.00 \mathrm{E}+00$ & $0.00 E+00$ & $0.00 \mathrm{E}+00$ & $0.00 \mathrm{E}+00$ & $0.00 \mathrm{E}+00$ & $0.00 \mathrm{E}+00$ & $0.00 \mathrm{E}+00$ & $0.00 \mathrm{E}+00$ & $0.00 \mathrm{E}+00$ & $0.00 \mathrm{E}+00$ & $0.00 \mathrm{E}+00$ & $0.00 \mathrm{E}+00$ & $0.00 \mathrm{E}+00$ & $0.00 \mathrm{E}+00$ & $0.00 \mathrm{E}+00$ & $0.00 \mathrm{E}+00$ \\
\hline $\mathrm{S} 16$ & $0.00 \mathrm{E}+00$ & $0.00 \mathrm{E}+00$ & $0.00 \mathrm{E}+00$ & $0.00 \mathrm{E}+00$ & $0.00 \mathrm{E}+00$ & $0.00 \mathrm{E}+00$ & $0.00 \mathrm{E}+00$ & $0.00 \mathrm{E}+00$ & $0.00 E+00$ & $0.00 \mathrm{E}+00$ & $0.00 E+00$ & $0.00 \mathrm{E}+00$ & $0.00 E+00$ & $0.00 \mathrm{E}+00$ & $0.00 \mathrm{E}+00$ & $0.00 \mathrm{E}+00$ \\
\hline S17 & $0.00 \mathrm{E}+00$ & $0.00 \mathrm{E}+00$ & $0.00 \mathrm{E}+00$ & $0.00 \mathrm{E}+00$ & $0.00 \mathrm{E}+00$ & $0.00 \mathrm{E}+00$ & $0.00 \mathrm{E}+00$ & $0.00 \mathrm{E}+00$ & $0.00 \mathrm{E}+00$ & $0.00 \mathrm{E}+00$ & $0.00 \mathrm{E}+00$ & $0.00 \mathrm{E}+00$ & $0.00 \mathrm{E}+00$ & $0.00 \mathrm{E}+00$ & $0.00 \mathrm{E}+00$ & $0.00 \mathrm{E}+00$ \\
\hline S18 & $2.79 \mathrm{E}-02$ & $3.62 \mathrm{E}-02$ & $2.54 \mathrm{E}+00$ & $6.86 \mathrm{E}-02$ & $6.15 \mathrm{E}+02$ & 2.27E-02 & 2.74E-03 & $6.28 \mathrm{E}-02$ & 1.84E-02 & $1.32 \mathrm{E}-04$ & 7.51E-03 & $6.21 \mathrm{E}-04$ & $1.24 \mathrm{E}-03$ & 5.62E-05 & $7.50 \mathrm{E}-03$ & $7.53 \mathrm{E}-03$ \\
\hline S19-O & $6.89 \mathrm{E}-01$ & $6.64 \mathrm{E}-01$ & $3.68 \mathrm{E}+01$ & $1.24 \mathrm{E}+00$ & $5.72 \mathrm{E}+04$ & $1.04 \mathrm{E}+00$ & 2.82E-01 & $3.12 \mathrm{E}-01$ & $2.05 \mathrm{E}-02$ & 1.41E-02 & $1.00 E-02$ & $6.42 \mathrm{E}-04$ & $1.45 E-03$ & $6.51 \mathrm{E}-04$ & 9.94E-03 & $1.01 E-02$ \\
\hline S19-A & $6.30 \mathrm{E}-01$ & $6.85 \mathrm{E}-01$ & $3.71 \mathrm{E}+01$ & $1.20 \mathrm{E}+00$ & $4.72 \mathrm{E}+04$ & $1.15 \mathrm{E}+00$ & $2.83 \mathrm{E}-01$ & $2.50 \mathrm{E}-01$ & $2.25 \mathrm{E}-02$ & 1.43E-02 & $1.00 E-02$ & $6.39 \mathrm{E}-04$ & $1.48 E-03$ & $6.55 \mathrm{E}-04$ & 9.94E-03 & $1.01 E-02$ \\
\hline S20-T & 1.52E-01 & 1.64E-01 & $8.85 \mathrm{E}+00$ & $3.01 \mathrm{E}-01$ & $2.49 \mathrm{E}+02$ & $1.93 \mathrm{E}-01$ & $9.54 \mathrm{E}-04$ & $5.65 \mathrm{E}-02$ & $3.49 E-02$ & $3.48 E-05$ & $3.48 E-02$ & 1.36E-03 & $2.61 \mathrm{E}-03$ & 1.16E-03 & $3.46 E-02$ & $3.50 E-02$ \\
\hline S20-B & $1.05 \mathrm{E}+01$ & $4.73 \mathrm{E}+00$ & $1.93 \mathrm{E}+02$ & $7.45 \mathrm{E}+00$ & $4.12 \mathrm{E}+04$ & $3.93 \mathrm{E}+00$ & 2.72E-01 & $2.21 \mathrm{E}+00$ & $3.75 \mathrm{E}-01$ & 1.32E-02 & $3.48 E-02$ & $4.26 \mathrm{E}-04$ & $3.47 E-02$ & $5.99 \mathrm{E}-03$ & 3.46E-02 & $3.50 E-02$ \\
\hline S21 & $2.24 \mathrm{E}-01$ & $2.59 \mathrm{E}-01$ & $2.04 \mathrm{E}+01$ & $5.88 \mathrm{E}-01$ & $7.68 \mathrm{E}+03$ & $3.41 \mathrm{E}-01$ & 2.46E-02 & $2.10 \mathrm{E}-01$ & $2.16 \mathrm{E}-02$ & $1.08 \mathrm{E}-03$ & $3.18 E-03$ & $2.59 \mathrm{E}-04$ & $2.24 E-03$ & 1.46E-03 & $3.16 E-03$ & $3.20 E-03$ \\
\hline $\mathrm{S} 22$ & $1.25 E+00$ & $1.25 E+00$ & $4.96 \mathrm{E}+01$ & $1.25 E+00$ & $5.91 \mathrm{E}+03$ & $4.97 E-01$ & $3.28 \mathrm{E}-02$ & $1.24 E+00$ & $1.25 E+00$ & $1.48 \mathrm{E}-03$ & $1.25 E+00$ & 4.18E-04 & 9.09E-03 & 6.93E-03 & $1.25 E+00$ & $1.25 E+00$ \\
\hline & & & & & & & & & & & & & & & & \\
\hline & & & & & & & & & & & & & & & & \\
\hline & & & & & & & & & & & & & & & & \\
\hline & & & & & & & & & & & & & & & & \\
\hline & & & & & & & & & & & & & & & & \\
\hline & & & & & & & & & & & & & & & & \\
\hline & & & & & & & & & & & & & & & & \\
\hline & & & & & & & & & & & & & & & & \\
\hline & & & & & & & & & & & & & & & & \\
\hline & & & & & & & & & & & & & & & & \\
\hline
\end{tabular}




\begin{tabular}{|c|c|c|c|c|c|c|c|c|c|c|c|c|c|c|}
\hline & & & & \\
\hline & & & & & & & & & & & & & & \\
\hline & & & & & & & & & & & & & & \\
\hline & & & & & & & & & & & & & & \\
\hline \multirow[b]{2}{*}{ Tank \# } & U-233 & U-234 & U-235 & U-236 & U-238 & Np-237 & Pu-238 & Pu-239 & Pu-240 & Pu-241 & Pu-242 & Am-241 & Am-243 & $\mathrm{Cm}-244$ \\
\hline & $\mathrm{g} /$ (L waste) & g/(L waste) & g/(L waste) & g/(L waste) & g/(L waste) & g/(L waste) & g/(L waste) & $\mathrm{g} /($ L waste $)$ & g/(L waste) & g/(L waste) & g/(L waste) & $\mathrm{g} /($ L waste $)$ & g/(L waste) & $\mathrm{g} /($ L waste $)$ \\
\hline S1 & $6.29 \mathrm{E}-05$ & $1.06 \mathrm{E}-03$ & $4.75 \mathrm{E}-02$ & 8.13E-04 & $6.75 \mathrm{E}+00$ & $4.88 \mathrm{E}-04$ & $4.17 \mathrm{E}-05$ & $3.36 \mathrm{E}-04$ & $1.30 \mathrm{E}-05$ & $2.01 \mathrm{E}-06$ & $4.61 E-06$ & $2.57 \mathrm{E}-06$ & $4.98 E-07$ & $7.54 \mathrm{E}-07$ \\
\hline S2 & $0.00 \mathrm{E}+00$ & $7.61 E-04$ & $8.80 \mathrm{E}-03$ & $3.62 \mathrm{E}-03$ & $3.25 \mathrm{E}+01$ & $1.83 \mathrm{E}-04$ & 9.96E-07 & $3.19 E-05$ & $0.00 \mathrm{E}+00$ & 7.97E-08 & 3.97E-04 & $9.79 \mathrm{E}-08$ & $6.55 \mathrm{E}-05$ & $3.51 \mathrm{E}-08$ \\
\hline S3 & $2.79 \mathrm{E}-04$ & $3.92 \mathrm{E}-04$ & 4.36E-02 & 7.29E-04 & $6.61 \mathrm{E}+00$ & $4.00 \mathrm{E}-04$ & 7.02E-07 & $2.54 \mathrm{E}-04$ & $4.63 E-06$ & $2.99 \mathrm{E}-07$ & 4.61E-06 & $3.34 E-08$ & $1.81 E-07$ & $1.53 \mathrm{E}-07$ \\
\hline S4 & 7.39E-04 & 7.36E-04 & 1.17E-01 & $1.74 \mathrm{E}-03$ & $2.38 \mathrm{E}+01$ & $3.40 \mathrm{E}-04$ & $1.36 E-06$ & 7.33E-04 & $7.36 E-04$ & $1.10 E-07$ & $7.38 E-04$ & $1.02 E-07$ & $5.70 E-07$ & $3.79 E-08$ \\
\hline S5 & 1.39E-03 & $2.46 \mathrm{E}-03$ & 1.87E-01 & 3.82E-03 & $3.25 \mathrm{E}+01$ & 7.02E-04 & 2.68E-04 & 4.39E-03 & 1.18E-03 & $1.10 \mathrm{E}-05$ & 7.00E-04 & 5.67E-05 & 7.00E-04 & 3.07E-03 \\
\hline S6 & $7.39 E-04$ & 7.36E-04 & 2.04E-02 & 7.37E-04 & $3.06 \mathrm{E}+00$ & $3.19 E-04$ & 9.43E-07 & 7.33E-04 & 7.36E-04 & 4.30E-07 & $7.38 E-04$ & 9.13E-08 & $5.25 E-07$ & 7.62E-09 \\
\hline S7 & 7.39E-04 & 7.36E-04 & 1.87E-01 & 3.69E-03 & $4.00 \mathrm{E}+01$ & 1.34E-03 & 1.30E-05 & 2.30E-03 & 7.36E-04 & 4.72E-06 & $7.38 E-04$ & 1.17E-05 & $4.28 E-07$ & $2.69 E-08$ \\
\hline S8 & 7.39E-04 & 7.36E-04 & 1.42E-02 & 7.37E-04 & $2.16 \mathrm{E}+00$ & 7.35E-04 & 2.01E-06 & 7.33E-04 & 7.36E-04 & 1.24E-05 & 7.38E-04 & $1.56 E-06$ & $8.75 E-06$ & $2.78 E-08$ \\
\hline S9 & $0.00 \mathrm{E}+00$ & $0.00 \mathrm{E}+00$ & $0.00 \mathrm{E}+00$ & $0.00 \mathrm{E}+00$ & $0.00 \mathrm{E}+00$ & $0.00 \mathrm{E}+00$ & $0.00 \mathrm{E}+00$ & $0.00 \mathrm{E}+00$ & $0.00 \mathrm{E}+00$ & $0.00 \mathrm{E}+00$ & $0.00 \mathrm{E}+00$ & $0.00 \mathrm{E}+00$ & $0.00 \mathrm{E}+00$ & $0.00 \mathrm{E}+00$ \\
\hline S10 & 1.47E-03 & 1.91E-03 & $1.34 \mathrm{E}-01$ & 3.62E-03 & $3.25 E+01$ & $1.20 \mathrm{E}-03$ & $1.45 \mathrm{E}-04$ & 3.32E-03 & $9.70 \mathrm{E}-04$ & 6.96E-06 & $3.97 \mathrm{E}-04$ & 3.28E-05 & 6.55E-05 & 2.97E-06 \\
\hline S11 & 7.01E-06 & 8.37E-06 & $1.12 \mathrm{E}-03$ & $8.44 \mathrm{E}-06$ & $1.45 E-01$ & 8.43E-06 & $1.77 \mathrm{E}-04$ & 8.39E-04 & $8.41 \mathrm{E}-04$ & $1.54 \mathrm{E}-05$ & $7.00 E-04$ & $1.08 E-06$ & $7.25 E-06$ & $7.54 \mathrm{E}-05$ \\
\hline $\mathrm{S} 12$ & $1.47 \mathrm{E}-03$ & $1.91 \mathrm{E}-03$ & $1.34 \mathrm{E}-01$ & $3.62 \mathrm{E}-03$ & $3.25 \mathrm{E}+01$ & $1.20 \mathrm{E}-03$ & $1.45 \mathrm{E}-04$ & $3.32 \mathrm{E}-03$ & 9.70E-04 & 6.96E-06 & 3.97E-04 & $3.28 \mathrm{E}-05$ & $6.55 \mathrm{E}-05$ & 2.97E-06 \\
\hline S13 & 1.07E-03 & $1.11 \mathrm{E}-03$ & 7.44E-02 & $3.19 \mathrm{E}-03$ & $1.20 \mathrm{E}+01$ & $6.96 E-04$ & 5.27E-05 & 7.33E-04 & 7.36E-04 & $2.75 \mathrm{E}-06$ & $7.38 E-04$ & 2.19E-07 & $1.16 E-06$ & $2.79 E-07$ \\
\hline $\mathrm{S} 14$ & $4.10 \mathrm{E}-05$ & $4.17 \mathrm{E}-04$ & $2.83 \mathrm{E}-02$ & 1.10E-03 & $1.32 \mathrm{E}+01$ & $1.71 \mathrm{E}-03$ & $7.58 \mathrm{E}-06$ & $2.11 \mathrm{E}-04$ & 8.39E-06 & $3.24 \mathrm{E}-07$ & $4.61 E-06$ & $4.58 \mathrm{E}-07$ & 5.82E-07 & 1.14E-07 \\
\hline $\mathrm{S} 15$ & $0.00 E+00$ & $0.00 E+00$ & $0.00 E+00$ & $0.00 E+00$ & $0.00 E+00$ & $0.00 E+00$ & $0.00 E+00$ & $0.00 E+00$ & $0.00 E+00$ & $0.00 E+00$ & $0.00 E+00$ & $0.00 E+00$ & $0.00 E+00$ & $0.00 E+00$ \\
\hline S16 & $0.00 E+00$ & $0.00 E+00$ & $0.00 E+00$ & $0.00 E+00$ & $0.00 \mathrm{E}+00$ & $0.00 \mathrm{E}+00$ & $0.00 \mathrm{E}+00$ & $0.00 \mathrm{E}+00$ & $0.00 E+00$ & $0.00 E+00$ & $0.00 \mathrm{E}+00$ & $0.00 E+00$ & $0.00 E+00$ & $0.00 \mathrm{E}+00$ \\
\hline $\mathrm{S} 17$ & $0.00 E+00$ & $0.00 E+00$ & $0.00 \mathrm{E}+00$ & $0.00 E+00$ & $0.00 \mathrm{E}+00$ & $0.00 \mathrm{E}+00$ & $0.00 \mathrm{E}+00$ & $0.00 E+00$ & $0.00 E+00$ & $0.00 E+00$ & $0.00 E+00$ & $0.00 \mathrm{E}+00$ & $0.00 E+00$ & $0.00 \mathrm{E}+00$ \\
\hline $\mathrm{S} 18$ & 1.47E-03 & $1.91 \mathrm{E}-03$ & $1.34 \mathrm{E}-01$ & 3.62E-03 & $3.25 E+01$ & $1.20 \mathrm{E}-03$ & $1.45 \mathrm{E}-04$ & 3.32E-03 & $9.70 \mathrm{E}-04$ & 6.96E-06 & 3.97E-04 & $3.28 \mathrm{E}-05$ & 6.55E-05 & 2.97E-06 \\
\hline S19-O & $3.17 \mathrm{E}-04$ & $3.06 \mathrm{E}-04$ & $1.69 \mathrm{E}-02$ & 5.71E-04 & $2.64 \mathrm{E}+01$ & 4.81E-04 & $1.30 \mathrm{E}-04$ & $1.44 \mathrm{E}-04$ & $9.44 \mathrm{E}-06$ & $6.51 \mathrm{E}-06$ & $4.61 E-06$ & $2.96 \mathrm{E}-07$ & $6.70 E-07$ & $3.00 \mathrm{E}-07$ \\
\hline S19-A & $2.90 \mathrm{E}-04$ & $3.15 \mathrm{E}-04$ & $1.71 \mathrm{E}-02$ & 5.51E-04 & $2.18 \mathrm{E}+01$ & $5.29 \mathrm{E}-04$ & $1.31 \mathrm{E}-04$ & 1.15E-04 & $1.04 \mathrm{E}-05$ & $6.58 \mathrm{E}-06$ & $4.61 E-06$ & $2.94 \mathrm{E}-07$ & $6.80 E-07$ & $3.02 E-07$ \\
\hline S20-T & $2.02 \mathrm{E}-05$ & 2.17E-05 & 1.17E-03 & 3.99E-05 & 3.30E-02 & $2.55 \mathrm{E}-05$ & $1.26 \mathrm{E}-07$ & 7.49E-06 & $4.63 E-06$ & $4.61 E-09$ & $4.61 E-06$ & $1.80 \mathrm{E}-07$ & $3.46 \mathrm{E}-07$ & $1.53 \mathrm{E}-07$ \\
\hline S20-B & $1.39 \mathrm{E}-03$ & $6.27 \mathrm{E}-04$ & $2.55 \mathrm{E}-02$ & 9.86E-04 & $5.46 \mathrm{E}+00$ & $5.21 \mathrm{E}-04$ & $3.60 \mathrm{E}-05$ & $2.92 \mathrm{E}-04$ & 4.97E-05 & $1.75 \mathrm{E}-06$ & $4.61 E-06$ & $5.64 \mathrm{E}-08$ & $4.60 E-06$ & 7.93E-07 \\
\hline S21 & $3.24 \mathrm{E}-04$ & $3.75 \mathrm{E}-04$ & $2.95 \mathrm{E}-02$ & $8.51 \mathrm{E}-04$ & $1.11 \mathrm{E}+01$ & $4.94 \mathrm{E}-04$ & $3.56 \mathrm{E}-05$ & 3.04E-04 & $3.12 \mathrm{E}-05$ & $1.56 \mathrm{E}-06$ & $4.61 E-06$ & $3.75 \mathrm{E}-07$ & $3.25 E-06$ & $2.12 \mathrm{E}-06$ \\
\hline $\mathrm{S} 22$ & 7.39E-04 & 7.36E-04 & $2.93 \mathrm{E}-02$ & 7.37E-04 & $3.49 \mathrm{E}+00$ & $2.94 E-04$ & $1.94 \mathrm{E}-05$ & 7.33E-04 & 7.36E-04 & $8.72 \mathrm{E}-07$ & 7.38E-04 & 2.47E-07 & $5.36 \mathrm{E}-06$ & $4.09 \mathrm{E}-06$ \\
\hline & & & & & & & & & & & & & & \\
\hline & & & & & & & & & & & & & & \\
\hline & & & & & & & & & & & & & & \\
\hline & & & & & & & & & & & & & & \\
\hline & & & & & & & & & & & & & & \\
\hline & & & & & & & & & & & & & & \\
\hline & & & & & & & & & & & & & & \\
\hline & & & & & & & & & & & & & & \\
\hline & & & & & & & & & & & & & & \\
\hline & & & & & & & & & & & & & & \\
\hline
\end{tabular}


Appendix 2

WSRC-TR-2000-00011

Revision 0

January 21, 2000

Page 33

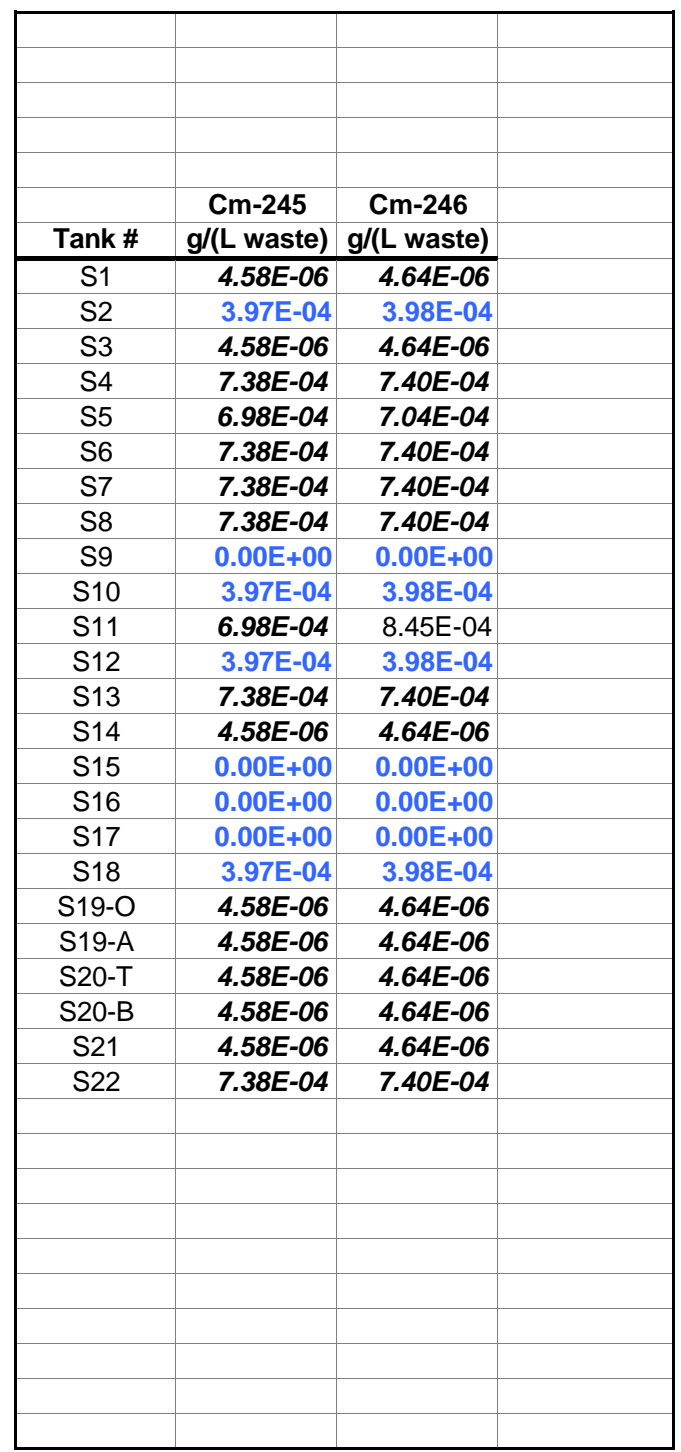




\begin{tabular}{|c|c|c|c|c|c|c|c|c|c|c|c|c|c|c|c|c|}
\hline \multicolumn{17}{|c|}{ Solvent Tank Nuclide Inventory Calculations - Suspended Solids } \\
\hline & $\mathrm{Ci} / \mathrm{g}$ & & $\mathrm{Ci} / \mathrm{g}$ & & $\mathrm{Ci} / \mathrm{g}$ & & $\mathrm{Ci} / \mathrm{g}$ & & $\mathrm{Ci} / \mathrm{g}$ & & $\mathrm{Ci} / \mathrm{g}$ & & $\mathrm{Ci} / \mathrm{g}$ & & $\mathrm{Ci} / \mathrm{g}$ & \\
\hline \multirow[t]{2}{*}{ SPCAT } & 9.64E-03 & & 6.24E-03 & & 2.16E-06 & & 6.47E-05 & & 3.36E-07 & & 7.05E-04 & & $1.71 \mathrm{E}+01$ & & $6.21 \mathrm{E}-02$ & \\
\hline & U-233 & Error & $\mathrm{U}-234$ & Error & U-235 & Error & $\mathrm{U}-236$ & Error & $\mathrm{U}-238$ & Error & Np-237 & Error & Pu-238 & Error & Pu-239 & Error \\
\hline Tank \# & $\mathrm{pCi} / \mathrm{g}$ & $\mathrm{pCi} / \mathrm{g}$ & $\mathrm{pCi} / \mathrm{g}$ & $\mathrm{pCi} / \mathrm{g}$ & $\mathrm{pCi} / \mathrm{g}$ & $\mathrm{pCi} / \mathrm{g}$ & $\mathrm{pCi} / \mathrm{g}$ & $\mathrm{pCi} / \mathrm{g}$ & $\mathrm{pCi} / \mathrm{g}$ & $\mathrm{pCi} / \mathrm{g}$ & $\mathrm{pCi} / \mathrm{g}$ & $\mathrm{pCi} / \mathrm{g}$ & $\mathrm{pCi} / \mathrm{g}$ & $\mathrm{pCi} / \mathrm{g}$ & $\mathrm{pCi} / \mathrm{g}$ & $\mathrm{pCi} / \mathrm{g}$ \\
\hline S1 & $6.35 E+05$ & $0.00 E+00$ & $6.49 E+05$ & $0.00 E+00$ & $2.28 \mathrm{E}+03$ & $4.57 \mathrm{E}+02$ & $6.76 E+03$ & $0.00 E+00$ & $5.27 \mathrm{E}+04$ & $1.05 \mathrm{E}+04$ & $7.34 E+04$ & $0.00 E+00$ & $9.32 \mathrm{E}+07$ & $2.80 \mathrm{E}+07$ & $6.49 E+06$ & $0.00 E+00$ \\
\hline S2 & $3.05 E+05$ & $0.00 E+00$ & $3.11 E+05$ & $0.00 E+00$ & $7.21 \mathrm{E}+03$ & $1.44 \mathrm{E}+03$ & $6.49 E+03$ & $0.00 E+00$ & $1.80 \mathrm{E}+05$ & $3.60 \mathrm{E}+04$ & $9.55 E+04$ & $0.00 E+00$ & $1.34 \mathrm{E}+08$ & $1.36 \mathrm{E}+07$ & $4.73 \mathrm{E}+06$ & $1.89 \mathrm{E}+06$ \\
\hline S3 & $2.91 E+04$ & $0.00 E+00$ & $1.48 \mathrm{E}+05$ & $2.96 \mathrm{E}+04$ & $6.67 \mathrm{E}+03$ & $1.33 \mathrm{E}+03$ & $2.92 \mathrm{E}+03$ & $5.84 \mathrm{E}+02$ & $1.36 \mathrm{E}+05$ & $2.73 E+04$ & $6.76 \mathrm{E}+04$ & $1.35 \mathrm{E}+04$ & $3.61 \mathrm{E}+07$ & $4.33 \mathrm{E}+06$ & $8.69 \mathrm{E}+06$ & $1.74 \mathrm{E}+06$ \\
\hline S4 & $4.55 \mathrm{E}+04$ & $9.10 \mathrm{E}+03$ & $1.21 \mathrm{E}+05$ & $2.42 \mathrm{E}+04$ & $4.21 \mathrm{E}+03$ & $8.42 \mathrm{E}+02$ & $2.28 \mathrm{E}+03$ & $4.57 \mathrm{E}+02$ & $8.60 \mathrm{E}+04$ & $1.72 \mathrm{E}+04$ & $4.03 \mathrm{E}+03$ & $8.05 \mathrm{E}+02$ & $3.18 \mathrm{E}+07$ & $6.36 \mathrm{E}+06$ & $3.15 \mathrm{E}+06$ & $6.31 \mathrm{E}+05$ \\
\hline S5 & $3.04 E+05$ & $0.00 E+00$ & $1.96 E+05$ & $0.00 E+00$ & $9.95 \mathrm{E}+02$ & $1.99 \mathrm{E}+02$ & $2.04 E+03$ & $0.00 E+00$ & $2.37 \mathrm{E}+04$ & $4.74 \mathrm{E}+03$ & $2.22 E+04$ & $0.00 E+00$ & $1.29 \mathrm{E}+08$ & $5.15 \mathrm{E}+07$ & $6.89 \mathrm{E}+06$ & $1.38 \mathrm{E}+06$ \\
\hline S6 & $4.55 \mathrm{E}+04$ & $9.10 \mathrm{E}+03$ & $1.51 \mathrm{E}+05$ & $3.03 E+04$ & $6.71 \mathrm{E}+03$ & $7.68 \mathrm{E}+02$ & $5.41 \mathrm{E}+03$ & $1.08 \mathrm{E}+03$ & $2.22 \mathrm{E}+05$ & $3.42 \mathrm{E}+04$ & $9.14 \mathrm{E}+03$ & $1.83 E+03$ & $3.24 \mathrm{E}+07$ & $9.73 \mathrm{E}+06$ & $5.50 \mathrm{E}+06$ & $1.10 \mathrm{E}+06$ \\
\hline S7 & $2.32 E+03$ & $0.00 E+00$ & $1.28 \mathrm{E}+05$ & $2.56 \mathrm{E}+04$ & $4.01 \mathrm{E}+03$ & $8.02 \mathrm{E}+02$ & $4.46 \mathrm{E}+03$ & 8.92E+02 & $1.45 \mathrm{E}+04$ & $2.90 \mathrm{E}+03$ & $3.59 \mathrm{E}+03$ & $7.18 \mathrm{E}+02$ & $3.50 \mathrm{E}+06$ & $2.66 \mathrm{E}+06$ & $5.45 \mathrm{E}+06$ & $1.09 \mathrm{E}+06$ \\
\hline S8 & $1.65 \mathrm{E}+04$ & $3.30 \mathrm{E}+03$ & $2.41 E+04$ & $4.81 \mathrm{E}+03$ & $2.48 \mathrm{E}+02$ & $4.96 \mathrm{E}+01$ & $2.50 \mathrm{E}+02$ & $5.00 \mathrm{E}+01$ & $5.41 \mathrm{E}+03$ & $5.41 \mathrm{E}+03$ & $1.61 \mathrm{E}+04$ & $3.22 \mathrm{E}+03$ & $8.60 \mathrm{E}+07$ & $1.72 \mathrm{E}+07$ & $1.16 \mathrm{E}+07$ & $2.32 \mathrm{E}+06$ \\
\hline S9 & $3.07 E+05$ & $1.42 \mathrm{E}+06$ & $1.63 E+05$ & $4.64 \mathrm{E}+05$ & $3.89 E+03$ & $8.88 \mathrm{E}+03$ & $4.63 E+03$ & $1.40 \mathrm{E}+04$ & $8.42 E+04$ & $1.72 \mathrm{E}+05$ & $1.08 \mathrm{E}+05$ & $5.36 \mathrm{E}+05$ & $1.61 \mathrm{E}+08$ & $5.70 \mathrm{E}+08$ & $9.95 \mathrm{E}+06$ & $3.40 E+07$ \\
\hline S10 & $3.07 E+05$ & $1.42 \mathrm{E}+06$ & $1.63 E+05$ & $4.64 \mathrm{E}+05$ & $3.89 E+03$ & $8.88 E+03$ & $4.63 E+03$ & $1.40 \mathrm{E}+04$ & $8.42 E+04$ & $1.72 \mathrm{E}+05$ & $1.08 E+05$ & $5.36 \mathrm{E}+05$ & $1.61 \mathrm{E}+08$ & $5.70 \mathrm{E}+08$ & $9.95 \mathrm{E}+06$ & $3.40 E+07$ \\
\hline S11 & $2.32 E+04$ & $0.00 E+00$ & $1.50 E+04$ & $0.00 E+00$ & $5.54 \mathrm{E}+01$ & $1.11 \mathrm{E}+01$ & $1.56 E+02$ & $0.00 E+00$ & $1.50 \mathrm{E}+03$ & $3.01 \mathrm{E}+02$ & $1.70 E+03$ & $0.00 E+00$ & $2.45 \mathrm{E}+07$ & $9.78 \mathrm{E}+06$ & $1.49 \mathrm{E}+06$ & $5.95 E+05$ \\
\hline S12 & $3.07 E+05$ & $1.42 \mathrm{E}+06$ & $1.63 \mathrm{E}+05$ & $4.64 \mathrm{E}+05$ & $3.89 E+03$ & $8.88 \mathrm{E}+03$ & $4.63 E+03$ & $1.40 \mathrm{E}+04$ & $8.42 E+04$ & $1.72 E+05$ & $1.08 \mathrm{E}+05$ & $5.36 \mathrm{E}+05$ & $1.61 \mathrm{E}+08$ & $5.70 \mathrm{E}+08$ & $9.95 \mathrm{E}+06$ & $3.40 E+07$ \\
\hline $\mathrm{S} 13$ & $1.92 \mathrm{E}+06$ & $3.85 \mathrm{E}+05$ & $7.66 \mathrm{E}+05$ & $1.53 \mathrm{E}+05$ & $1.67 \mathrm{E}+04$ & $3.34 \mathrm{E}+03$ & $2.28 \mathrm{E}+04$ & $4.57 \mathrm{E}+03$ & $2.55 E+05$ & $2.55 \mathrm{E}+05$ & $1.20 \mathrm{E}+05$ & $2.40 \mathrm{E}+04$ & 1.13E+09 & $2.26 \mathrm{E}+08$ & $6.58 \mathrm{E}+07$ & $1.32 \mathrm{E}+07$ \\
\hline S14 & $2.54 E+04$ & $0.00 E+00$ & $2.58 E+04$ & $0.00 E+00$ & $1.80 \mathrm{E}+02$ & $3.60 \mathrm{E}+01$ & $2.68 E+02$ & $0.00 E+00$ & $4.48 \mathrm{E}+03$ & $8.95 E+02$ & $3.41 E+03$ & $1.36 \mathrm{E}+03$ & $2.48 \mathrm{E}+08$ & $2.68 \mathrm{E}+07$ & $1.57 \mathrm{E}+06$ & $3.14 \mathrm{E}+05$ \\
\hline S15 & $3.07 E+05$ & $1.42 \mathrm{E}+06$ & $1.63 \mathrm{E}+05$ & $4.64 \mathrm{E}+05$ & $3.89 E+03$ & $8.88 \mathrm{E}+03$ & $4.63 E+03$ & $1.40 \mathrm{E}+04$ & $8.42 E+04$ & $1.72 \mathrm{E}+05$ & $1.08 E+05$ & $5.36 \mathrm{E}+05$ & $1.61 \mathrm{E}+08$ & $5.70 \mathrm{E}+08$ & $9.95 \mathrm{E}+06$ & $3.40 E+07$ \\
\hline S16 & $3.07 E+05$ & $1.42 \mathrm{E}+06$ & $1.63 E+05$ & $4.64 \mathrm{E}+05$ & $3.89 E+03$ & $8.88 \mathrm{E}+03$ & $4.63 E+03$ & $1.40 \mathrm{E}+04$ & $8.42 E+04$ & $1.72 \mathrm{E}+05$ & $1.08 E+05$ & $5.36 \mathrm{E}+05$ & $1.61 \mathrm{E}+08$ & $5.70 \mathrm{E}+08$ & $9.95 \mathrm{E}+06$ & $3.40 E+07$ \\
\hline S17 & $3.07 E+05$ & $1.42 \mathrm{E}+06$ & $1.63 E+05$ & $4.64 \mathrm{E}+05$ & $3.89 E+03$ & $8.88 \mathrm{E}+03$ & $4.63 E+03$ & $1.40 \mathrm{E}+04$ & $8.42 E+04$ & $1.72 \mathrm{E}+05$ & $1.08 \mathrm{E}+05$ & $5.36 \mathrm{E}+05$ & $1.61 \mathrm{E}+08$ & $5.70 \mathrm{E}+08$ & $9.95 \mathrm{E}+06$ & $3.40 E+07$ \\
\hline S18 & $3.07 E+05$ & $1.42 \mathrm{E}+06$ & $1.63 E+05$ & $4.64 \mathrm{E}+05$ & $3.89 E+03$ & $8.88 \mathrm{E}+03$ & $4.63 E+03$ & $1.40 \mathrm{E}+04$ & $8.42 E+04$ & $1.72 \mathrm{E}+05$ & $1.08 E+05$ & $5.36 \mathrm{E}+05$ & $1.61 \mathrm{E}+08$ & $5.70 \mathrm{E}+08$ & $9.95 \mathrm{E}+06$ & $3.40 E+07$ \\
\hline S19-O & $3.93 E+04$ & $1.57 \mathrm{E}+04$ & $6.85 E+04$ & 1.37E+04 & $1.90 \mathrm{E}+03$ & $3.79 \mathrm{E}+02$ & $1.59 \mathrm{E}+03$ & $3.19 \mathrm{E}+02$ & $4.59 \mathrm{E}+04$ & $9.19 \mathrm{E}+03$ & $9.10 \mathrm{E}+05$ & $3.42 \mathrm{E}+05$ & $9.19 \mathrm{E}+07$ & $9.19 \mathrm{E}+06$ & $2.21 \mathrm{E}+06$ & $4.41 \mathrm{E}+05$ \\
\hline S19-A & $4.48 \mathrm{E}+04$ & $1.79 \mathrm{E}+04$ & $4.55 \mathrm{E}+04$ & $1.82 \mathrm{E}+04$ & $1.06 \mathrm{E}+03$ & $2.12 \mathrm{E}+02$ & $9.50 \mathrm{E}+02$ & $1.90 \mathrm{E}+02$ & $2.64 \mathrm{E}+04$ & $5.28 \mathrm{E}+03$ & $1.40 \mathrm{E}+04$ & $2.79 E+03$ & $2.29 \mathrm{E}+07$ & $2.29 E+06$ & $6.94 \mathrm{E}+05$ & $2.77 \mathrm{E}+05$ \\
\hline S20-T & $3.03 E+04$ & $0.00 E+00$ & $3.08 E+04$ & $0.00 E+00$ & $2.28 \mathrm{E}+01$ & $9.10 \mathrm{E}+00$ & $3.21 E+02$ & $0.00 E+00$ & $6.05 \mathrm{E}+02$ & $1.21 \mathrm{E}+02$ & $3.51 E+03$ & $0.00 E+00$ & $8.63 E+05$ & $4.32 \mathrm{E}+05$ & $3.08 E+05$ & $0.00 E+00$ \\
\hline S20-B & $2.03 E+05$ & $0.00 E+00$ & $2.06 E+05$ & $0.00 E+00$ & $1.41 \mathrm{E}+03$ & $2.81 \mathrm{E}+02$ & $2.15 E+03$ & $0.00 E+00$ & $3.73 E+04$ & 7.47E+03 & $2.56 \mathrm{E}+04$ & $1.03 E+04$ & $8.83 \mathrm{E}+07$ & $8.83 E+06$ & $3.10 \mathrm{E}+06$ & $1.24 \mathrm{E}+06$ \\
\hline $\mathrm{S} 21$ & $4.68 \mathrm{E}+04$ & $9.37 \mathrm{E}+03$ & $3.89 \mathrm{E}+04$ & $7.78 \mathrm{E}+03$ & $1.32 \mathrm{E}+03$ & $2.64 \mathrm{E}+02$ & $1.40 \mathrm{E}+03$ & $2.79 \mathrm{E}+02$ & $3.21 \mathrm{E}+04$ & $6.41 \mathrm{E}+03$ & $2.92 \mathrm{E}+04$ & $5.85 \mathrm{E}+03$ & $6.85 \mathrm{E}+07$ & $1.78 \mathrm{E}+07$ & $3.64 \mathrm{E}+06$ & $7.28 \mathrm{E}+05$ \\
\hline S22 & $3.53 \mathrm{E}+04$ & $7.06 \mathrm{E}+03$ & $2.40 \mathrm{E}+04$ & $4.80 \mathrm{E}+03$ & $6.62 \mathrm{E}+02$ & $1.32 \mathrm{E}+02$ & $5.81 \mathrm{E}+02$ & $1.16 \mathrm{E}+02$ & $1.39 E+04$ & $0.00 E+00$ & $3.99 \mathrm{E}+03$ & $7.98 \mathrm{E}+02$ & $7.48 \mathrm{E}+07$ & $4.49 \mathrm{E}+07$ & $7.03 E+06$ & $1.41 \mathrm{E}+06$ \\
\hline \multicolumn{17}{|c|}{ Values listed in Black Bold ltalics print are detection limits or upper limits. } \\
\hline \multicolumn{17}{|c|}{ Tanks S8and S13 U-238 activity is a lower limit. Uncertainty of $100 \%$ has been assigned. } \\
\hline \multicolumn{17}{|c|}{\begin{tabular}{|l|l|l|l|} 
The values calculated for samples S20-T and S20-B are upper limits. & & & \\
\end{tabular}} \\
\hline \multicolumn{17}{|c|}{ Values listed in Blue Bold Italics are calculated based upon average activity concentrations of the analyzed suspended solids from aqueous solutions. } \\
\hline \multirow{2}{*}{\multicolumn{17}{|c|}{$\begin{array}{l}\text { The suspended solids from the organic liquids in Tanks S11 and S20 (20-T) were not used to calculate average activity concentrations. } \\
\text { Since S19-O and S19-A represent duplicate samples, only S19-O was used in the average activity calculations. }\end{array}$}} \\
\hline & & & & & & & & & & & & & & & & \\
\hline \multicolumn{17}{|c|}{\begin{tabular}{l|l|l|} 
Since S19-O and S19-A represent duplicate samples, only S19-O was used in the average activity calculations. & \\
The average $\mathrm{Cm}-245$ value is based upon the activity concentration from the suspended solids in Tank S6 only.
\end{tabular}} \\
\hline \multicolumn{17}{|c|}{ The average $\mathrm{Cm}-246$ value is based upon the activity concentration from the supsended solids in Tanks S6, S21, and S22. } \\
\hline Errors as & ssociated wit & th average a & ctivities were & calculated & by averaging & the relative & eiros asso & 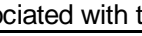 & . & & & & & & & \\
\hline
\end{tabular}




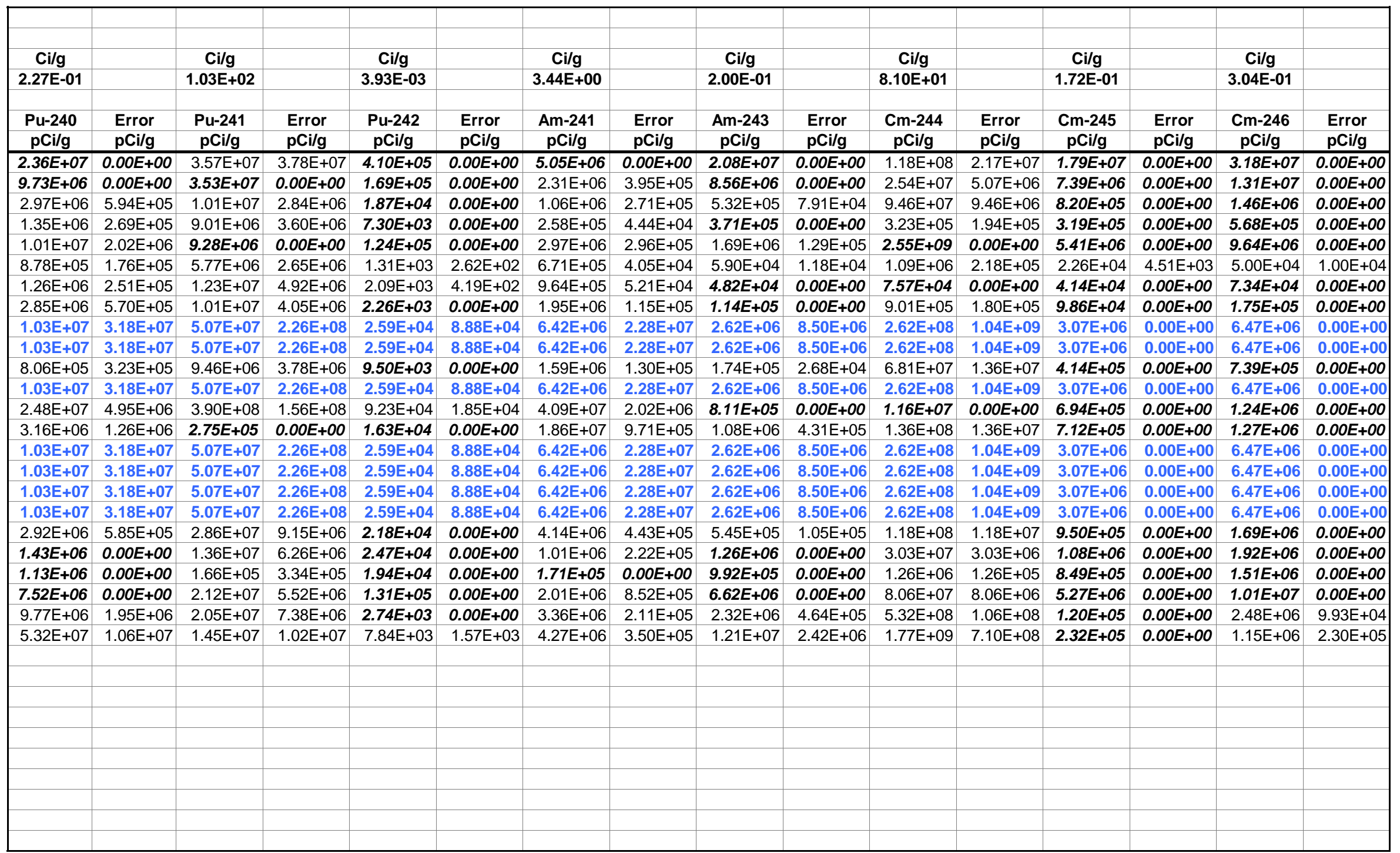




\begin{tabular}{|c|c|c|c|c|c|c|c|c|c|c|c|c|c|c|c|c|}
\hline \multicolumn{17}{|c|}{ Total Activity Including Error } \\
\hline & & & & & & & & & & & & & & & & \\
\hline & & & & & & & & & & & & & & & & \\
\hline & & & & & & & & & & & & & & & & \\
\hline Mass & U-233 & U-234 & U-235 & U-236 & U-238 & Np-237 & Pu-238 & Pu-239 & Pu-240 & Pu-241 & Pu-242 & Am-241 & Am-243 & $\mathrm{Cm}-244$ & $\mathrm{Cm}-245$ & $\mathrm{Cm}-246$ \\
\hline Grams & $\mathbf{C i}$ & $\mathbf{C i}$ & $\mathbf{C i}$ & $\mathbf{C i}$ & $\mathbf{C i}$ & $\mathrm{Ci}$ & $\mathrm{Ci}$ & $\mathrm{Ci}$ & $\mathrm{Ci}$ & $\mathrm{Ci}$ & $\mathrm{Ci}$ & $\mathbf{C i}$ & $\mathrm{Ci}$ & $\mathrm{Ci}$ & $\mathrm{Ci}$ & $\mathrm{Ci}$ \\
\hline 38.79 & $2.46 E-05$ & $2.52 E-05$ & 1.06E-07 & $2.62 E-07$ & $2.45 E-06$ & $2.85 E-06$ & $4.70 \mathrm{E}-03$ & $2.52 E-04$ & $9.15 E-04$ & $2.85 E-03$ & $1.59 \mathrm{E}-05$ & 1.96E-04 & 8.07E-04 & 5.42E-03 & $6.94 E-04$ & $1.23 E-03$ \\
\hline 84.82 & $2.59 E-05$ & $2.64 E-05$ & 7.34E-07 & $5.50 E-07$ & $1.83 \mathrm{E}-05$ & $8.10 E-06$ & $1.25 \mathrm{E}-02$ & 5.62E-04 & $8.25 E-04$ & $2.99 E-03$ & $1.43 E-05$ & $2.29 E-04$ & $7.26 E-04$ & $2.58 \mathrm{E}-03$ & $6.27 E-04$ & $1.11 E-03$ \\
\hline 2197.27 & 6.39E-05 & $3.90 \mathrm{E}-04$ & 1.76E-05 & 7.70E-06 & 3.59E-04 & 1.78E-04 & 8.88E-02 & 2.29E-02 & 7.83E-03 & 2.84E-02 & 4.11E-05 & 2.92E-03 & 1.34E-03 & 2.29E-01 & $1.80 E-03$ & $3.21 E-03$ \\
\hline 301.60 & $1.65 \mathrm{E}-05$ & $4.38 \mathrm{E}-05$ & $1.52 \mathrm{E}-06$ & $8.25 \mathrm{E}-07$ & $3.11 \mathrm{E}-05$ & $1.46 \mathrm{E}-06$ & $1.15 \mathrm{E}-02$ & $1.14 \mathrm{E}-03$ & $4.88 \mathrm{E}-04$ & $3.80 \mathrm{E}-03$ & $2.20 E-06$ & $9.12 \mathrm{E}-05$ & $1.12 E-04$ & $1.56 \mathrm{E}-04$ & $9.62 E-05$ & $1.71 E-04$ \\
\hline 3.48 & $1.06 E-06$ & $6.82 E-07$ & 4.16E-09 & 7.10E-09 & 9.90E-08 & 7.73E-08 & 6.28E-04 & 2.88E-05 & 4.22E-05 & $3.23 E-05$ & 4.32E-07 & 1.14E-05 & 6.33E-06 & 8.87E-03 & $1.88 E-05$ & $3.35 E-05$ \\
\hline 16503.08 & 9.01E-04 & 2.99E-03 & 1.23E-04 & 1.07E-04 & 4.23E-03 & 1.81E-04 & 6.95E-01 & 1.09E-01 & 1.74E-02 & $1.39 \mathrm{E}-01$ & $2.59 \mathrm{E}-05$ & 1.17E-02 & 1.17E-03 & 2.16E-02 & 4.47E-04 & 9.90E-04 \\
\hline 2494.27 & $5.79 E-06$ & 3.83E-04 & 1.20E-05 & 1.33E-05 & 4.34E-05 & 1.07E-05 & 1.54E-02 & 1.63E-02 & 3.77E-03 & 4.30E-02 & $6.26 \mathrm{E}-06$ & $2.53 \mathrm{E}-03$ & $1.20 E-04$ & 1.89E-04 & $1.03 E-04$ & $1.83 E-04$ \\
\hline 490.55 & $9.71 \mathrm{E}-06$ & 1.42E-05 & 1.46E-07 & 1.47E-07 & 5.31E-06 & $9.48 \mathrm{E}-06$ & 5.06E-02 & 6.83E-03 & 1.68E-03 & 6.94E-03 & 1.11E-06 & 1.01E-03 & 5.59E-05 & 5.30E-04 & 4.84E-05 & 8.58E-05 \\
\hline 0 & $0.00 E+00$ & $0.00 \mathrm{E}+00$ & $0.00 E+00$ & $0.00 E+00$ & $0.00 E+00$ & $0.00 E+00$ & $0.00 E+00$ & $0.00 E+00$ & $0.00 E+00$ & $0.00 \mathrm{E}+00$ & $0.00 \mathrm{E}+00$ & $0.00 E+00$ & $0.00 E+00$ & $0.00 E+00$ & $0.00 E+00$ & $0.00 E+00$ \\
\hline 84.33 & 1.46E-04 & 5.29E-05 & 1.08E-06 & 1.57E-06 & 2.16E-05 & 5.43E-05 & 6.16E-02 & 3.71E-03 & $3.55 \mathrm{E}-03$ & 2.33E-02 & 9.67E-06 & 2.46E-03 & 9.38E-04 & 1.10E-01 & 2.59E-04 & 5.46E-04 \\
\hline 3.79 & $8.79 E-08$ & 5.69E-08 & $2.52 \mathrm{E}-10$ & 5.91E-10 & 6.83E-09 & 6.44E-09 & 1.30E-04 & 7.90E-06 & $4.28 \mathrm{E}-06$ & 5.02E-05 & $3.60 E-08$ & 6.52E-06 & 7.61E-07 & $3.10 \mathrm{E}-04$ & $1.57 E-06$ & 2.80E-06 \\
\hline 42.17 & 7.28E-05 & 2.64E-05 & 5.39E-07 & 7.86E-07 & $1.08 \mathrm{E}-05$ & 2.72E-05 & 3.08E-02 & 1.85E-03 & 1.78E-03 & 1.17E-02 & $4.84 E-06$ & 1.23E-03 & $4.69 E-04$ & 5.49E-02 & $1.29 E-04$ & $2.73 E-04$ \\
\hline 181.14 & 4.18E-04 & $1.66 \mathrm{E}-04$ & 3.63E-06 & 4.96E-06 & 9.24E-05 & 2.61E-05 & $2.46 \mathrm{E}-01$ & 1.43E-02 & 5.39E-03 & 9.89E-02 & 2.01E-05 & 7.77E-03 & $1.47 E-04$ & $2.10 E-03$ & $1.26 E-04$ & $2.25 E-04$ \\
\hline 254.4 & 6.46E-06 & $6.56 E-06$ & 5.50E-08 & $6.82 E-08$ & 1.37E-06 & $1.21 \mathrm{E}-06$ & 6.99E-02 & 4.79E-04 & 1.12E-03 & 7.00E-05 & $4.15 E-06$ & 4.98E-03 & 3.84E-04 & 3.81E-02 & $1.81 E-04$ & 3.23E-04 \\
\hline 0 & $0.00 E+00$ & $0.00 \mathrm{E}+00$ & $0.00 \mathrm{E}+00$ & $0.00 \mathrm{E}+00$ & $0.00 E+00$ & $0.00 E+00$ & $0.00 E+00$ & $0.00 E+00$ & $0.00 \mathrm{E}+00$ & $0.00 \mathrm{E}+00$ & $0.00 \mathrm{E}+00$ & $0.00 E+00$ & $0.00 E+00$ & $0.00 E+00$ & $0.00 E+00$ & $0.00 E+00$ \\
\hline 0 & $0.00 E+00$ & $0.00 E+00$ & $0.00 E+00$ & $0.00 E+00$ & $0.00 E+00$ & $0.00 E+00$ & $0.00 E+00$ & $0.00 E+00$ & $0.00 E+00$ & $0.00 \mathrm{E}+00$ & $0.00 \mathrm{E}+00$ & $0.00 E+00$ & $0.00 E+00$ & $0.00 E+00$ & $0.00 E+00$ & $0.00 E+00$ \\
\hline 0 & $0.00 E+00$ & $0.00 E+00$ & $0.00 \mathrm{E}+00$ & $0.00 \mathrm{E}+00$ & $0.00 E+00$ & $0.00 E+00$ & $0.00 E+00$ & $0.00 E+00$ & $0.00 \mathrm{E}+00$ & $0.00 \mathrm{E}+00$ & $0.00 \mathrm{E}+00$ & $0.00 \mathrm{E}+00$ & $0.00 \mathrm{E}+00$ & $0.00 E+00$ & $0.00 E+00$ & $0.00 E+00$ \\
\hline 42.17 & 7.28E-05 & 2.64E-05 & 5.39E-07 & 7.86E-07 & 1.08E-05 & 2.72E-05 & 3.08E-02 & 1.85E-03 & 1.78E-03 & 1.17E-02 & 4.84E-06 & 1.23E-03 & 4.69E-04 & 5.49E-02 & 1.29E-04 & 2.73E-04 \\
\hline 2849.5 & 1.57E-04 & 2.34E-04 & 6.49E-06 & 5.44E-06 & 1.57E-04 & 3.57E-03 & 2.88E-01 & 7.55E-03 & 9.99E-03 & $1.08 \mathrm{E}-01$ & $6.21 E-05$ & 1.31E-02 & 1.85E-03 & 3.70E-01 & $2.71 E-03$ & $4.82 E-03$ \\
\hline 2619.21 & 1.64E-04 & 1.67E-04 & 3.33E-06 & 2.99E-06 & 8.30E-05 & 4.40E-05 & 6.60E-02 & 2.54E-03 & $3.75 E-03$ & 5.20E-02 & 6.47E-05 & 3.23E-03 & 3.30E-03 & 8.73E-02 & $2.83 E-03$ & $5.03 E-03$ \\
\hline 11119.41 & 3.37E-04 & $3.42 E-04$ & $3.55 E-07$ & 3.57E-06 & 8.07E-06 & 3.90E-05 & 1.44E-02 & $3.42 E-03$ & $1.26 E-02$ & 5.56E-03 & $2.16 E-04$ & $1.90 E-03$ & 1.10E-02 & 1.54E-02 & $9.44 E-03$ & $1.68 E-02$ \\
\hline 1587.41 & 3.22E-04 & 3.27E-04 & 2.68E-06 & $3.41 E-06$ & 7.11E-05 & 5.70E-05 & 1.54E-01 & 6.89E-03 & 1.19E-02 & 4.24E-02 & $2.08 E-04$ & 4.54E-03 & $1.05 E-02$ & $1.41 \mathrm{E}-01$ & 8.37E-03 & $1.60 E-02$ \\
\hline 7099.02 & 3.99E-04 & 3.31E-04 & 1.12E-05 & 1.19E-05 & 2.73E-04 & 2.49E-04 & 6.13E-01 & 3.10E-02 & 8.32E-02 & 1.98E-01 & $1.95 E-05$ & 2.54E-02 & 1.98E-02 & $4.53 E+00$ & $8.52 E-04$ & 1.83E-02 \\
\hline 541.85 & 2.30E-05 & 1.56E-05 & 4.30E-07 & 3.78E-07 & 7.53E-06 & 2.59E-06 & 6.49E-02 & 4.57E-03 & $3.46 \mathrm{E}-02$ & 1.34E-02 & $5.10 \mathrm{E}-06$ & $2.50 \mathrm{E}-03$ & 7.87E-03 & $1.34 \mathrm{E}+00$ & $1.26 E-04$ & 7.48E-04 \\
\hline & & & & & & & & & & & & & & & & \\
\hline & & & & & & & & & & & & & & & & \\
\hline & & & & & & & & & & & & & & & & \\
\hline & & & & & & & & & & & & & & & & \\
\hline & & & & & & & & & & & & & & & & \\
\hline & & & & & & & & & & & & & & & & \\
\hline & & & & & & & & & & & & & & & & \\
\hline & & & & & & & & & & & & & & & & \\
\hline & & & & & & & & & & & & & & & & \\
\hline & & & & & & & & & & & & & & & & \\
\hline
\end{tabular}




\begin{tabular}{|c|c|c|c|c|c|c|c|c|c|c|c|c|c|c|c|c|}
\hline \multicolumn{17}{|c|}{ Activity Concentration Including Error } \\
\hline & & & & & & & & & & & & & & & & \\
\hline & & & & & & & & & & & & & & & & \\
\hline & & & & & & & & & & & & & & & & \\
\hline & U-233 & U-234 & U-235 & U-236 & U-238 & Np-237 & Pu-238 & Pu-239 & Pu-240 & Pu-241 & Pu-242 & Am-241 & Am-243 & $\mathrm{Cm}-244$ & $\mathrm{Cm}-245$ & $\mathrm{Cm}-246$ \\
\hline Tank \# & $\mathrm{Ci} / \mathrm{g}$ & $\mathrm{Ci} / \mathrm{g}$ & $\mathrm{Ci} / \mathrm{g}$ & $\mathrm{Ci} / \mathrm{g}$ & $\mathrm{Ci} / \mathrm{g}$ & $\mathrm{Ci} / \mathrm{g}$ & $\mathrm{Ci} / \mathrm{g}$ & $\mathrm{Ci} / \mathrm{g}$ & $\mathrm{Ci} / \mathrm{g}$ & $\mathrm{Ci} / \mathrm{g}$ & $\mathrm{Ci} / \mathrm{g}$ & $\mathrm{Ci} / \mathrm{g}$ & $\mathrm{Ci} / \mathrm{g}$ & $\mathrm{Ci} / \mathrm{g}$ & $\mathrm{Ci} / \mathrm{g}$ & $\mathrm{Ci} / \mathrm{g}$ \\
\hline S1 & $6.35 E-07$ & $6.49 E-07$ & $2.74 \mathrm{E}-09$ & $6.76 E-09$ & $6.32 \mathrm{E}-08$ & $7.34 E-08$ & $1.21 \mathrm{E}-04$ & $6.49 E-06$ & $2.36 E-05$ & $7.35 \mathrm{E}-05$ & $4.10 \mathrm{E}-07$ & $5.05 E-06$ & $2.08 E-05$ & $1.40 \mathrm{E}-04$ & $1.79 E-05$ & $3.18 E-05$ \\
\hline S2 & 3.05E-07 & $3.11 E-07$ & 8.65E-09 & 6.49E-09 & 2.16E-07 & 9.55E-08 & $1.48 \mathrm{E}-04$ & 6.62E-06 & 9.73E-06 & 3.53E-05 & 1.69E-07 & 2.71E-06 & 8.56E-06 & 3.05E-05 & 7.39E-06 & 1.31E-05 \\
\hline S3 & $2.91 E-08$ & 1.78E-07 & 8.00E-09 & 3.50E-09 & 1.63E-07 & $8.11 \mathrm{E}-08$ & 4.04E-05 & 1.04E-05 & 3.56E-06 & 1.29E-05 & 1.87E-08 & 1.33E-06 & 6.11E-07 & 1.04E-04 & 8.20E-07 & 1.46E-06 \\
\hline S4 & 5.46E-08 & 1.45E-07 & 5.05E-09 & 2.74E-09 & 1.03E-07 & 4.84E-09 & 3.82E-05 & 3.78E-06 & 1.62E-06 & 1.26E-05 & 7.30E-09 & 3.02E-07 & 3.71E-07 & 5.17E-07 & $3.19 E-07$ & $5.68 E-07$ \\
\hline S5 & $3.04 E-07$ & 1.96E-07 & 1.19E-09 & $2.04 E-09$ & 2.84E-08 & $2.22 E-08$ & $1.81 \mathrm{E}-04$ & 8.27E-06 & $1.21 \mathrm{E}-05$ & $9.28 E-06$ & 1.24E-07 & 3.27E-06 & 1.82E-06 & $2.55 E-03$ & 5.41E-06 & 9.64E-06 \\
\hline S6 & $5.46 \mathrm{E}-08$ & 1.81E-07 & 7.48E-09 & 6.49E-09 & 2.56E-07 & 1.10E-08 & $4.21 \mathrm{E}-05$ & $6.60 \mathrm{E}-06$ & $1.05 \mathrm{E}-06$ & 8.42E-06 & 1.57E-09 & 7.12E-07 & 7.08E-08 & $1.31 \mathrm{E}-06$ & 2.71E-08 & $6.00 \mathrm{E}-08$ \\
\hline S7 & $2.32 E-09$ & 1.54E-07 & 4.81E-09 & 5.35E-09 & 1.74E-08 & 4.31E-09 & 6.16E-06 & $6.54 \mathrm{E}-06$ & $1.51 \mathrm{E}-06$ & 1.72E-05 & 2.51E-09 & 1.02E-06 & $4.82 E-08$ & 7.57E-08 & $4.14 E-08$ & 7.34E-08 \\
\hline S8 & $1.98 \mathrm{E}-08$ & 2.89E-08 & $2.98 \mathrm{E}-10$ & $3.00 \mathrm{E}-10$ & 1.08E-08 & 1.93E-08 & 1.03E-04 & 1.39E-05 & $3.42 \mathrm{E}-06$ & 1.42E-05 & 2.26E-09 & 2.07E-06 & 1.14E-07 & 1.08E-06 & $9.86 E-08$ & $1.75 E-07$ \\
\hline S9 & $0.00 \mathrm{E}+00$ & $0.00 \mathrm{E}+00$ & $0.00 \mathrm{E}+00$ & $0.00 \mathrm{E}+00$ & $0.00 \mathrm{E}+00$ & $0.00 \mathrm{E}+00$ & $0.00 \mathrm{E}+00$ & $0.00 \mathrm{E}+00$ & $0.00 \mathrm{E}+00$ & $0.00 \mathrm{E}+00$ & $0.00 \mathrm{E}+00$ & $0.00 \mathrm{E}+00$ & $0.00 \mathrm{E}+00$ & $0.00 \mathrm{E}+00$ & $0.00 \mathrm{E}+00$ & $0.00 \mathrm{E}+00$ \\
\hline S10 & 1.73E-06 & 6.27E-07 & 1.28E-08 & 1.86E-08 & 2.56E-07 & 6.44E-07 & 7.31E-04 & 4.40E-05 & 4.21E-05 & 2.77E-04 & 1.15E-07 & 2.92E-05 & 1.11E-05 & 1.30E-03 & 3.07E-06 & 6.47E-06 \\
\hline S11 & $2.32 E-08$ & $1.50 E-08$ & $6.65 \mathrm{E}-11$ & $1.56 E-10$ & 1.80E-09 & $1.70 E-09$ & 3.43E-05 & 2.09E-06 & 1.13E-06 & 1.32E-05 & 9.50E-09 & 1.72E-06 & 2.01E-07 & 8.17E-05 & $4.14 E-07$ & 7.39E-07 \\
\hline S12 & 1.73E-06 & 6.27E-07 & $1.28 \mathrm{E}-08$ & 1.86E-08 & 2.56E-07 & 6.44E-07 & 7.31E-04 & 4.40E-05 & 4.21E-05 & 2.77E-04 & 1.15E-07 & 2.92E-05 & 1.11E-05 & 1.30E-03 & 3.07E-06 & 6.47E-06 \\
\hline $\mathrm{S} 13$ & 2.31E-06 & 9.19E-07 & 2.00E-08 & 2.74E-08 & $5.10 \mathrm{E}-07$ & $1.44 \mathrm{E}-07$ & 1.36E-03 & $7.90 \mathrm{E}-05$ & $2.98 \mathrm{E}-05$ & 5.46E-04 & 1.11E-07 & 4.29E-05 & 8.11E-07 & 1.16E-05 & $6.94 E-07$ & $1.24 E-06$ \\
\hline S14 & $2.54 E-08$ & $2.58 E-08$ & $2.16 \mathrm{E}-10$ & $2.68 E-10$ & 5.38E-09 & 4.77E-09 & 2.75E-04 & $1.88 \mathrm{E}-06$ & $4.42 \mathrm{E}-06$ & $2.75 E-07$ & $1.63 E-08$ & 1.96E-05 & 1.51E-06 & 1.50E-04 & 7.12E-07 & 1.27E-06 \\
\hline S15 & $0.00 \mathrm{E}+00$ & $0.00 \mathrm{E}+00$ & $0.00 \mathrm{E}+00$ & $0.00 \mathrm{E}+00$ & $0.00 \mathrm{E}+00$ & $0.00 \mathrm{E}+00$ & $0.00 \mathrm{E}+00$ & $0.00 \mathrm{E}+00$ & $0.00 \mathrm{E}+00$ & $0.00 \mathrm{E}+00$ & $0.00 \mathrm{E}+00$ & $0.00 \mathrm{E}+00$ & $0.00 E+00$ & $0.00 \mathrm{E}+00$ & $0.00 \mathrm{E}+00$ & $0.00 \mathrm{E}+00$ \\
\hline S16 & $0.00 \mathrm{E}+00$ & $0.00 \mathrm{E}+00$ & $0.00 \mathrm{E}+00$ & $0.00 \mathrm{E}+00$ & $0.00 \mathrm{E}+00$ & $0.00 \mathrm{E}+00$ & $0.00 \mathrm{E}+00$ & $0.00 \mathrm{E}+00$ & $0.00 E+00$ & $0.00 \mathrm{E}+00$ & $0.00 E+00$ & $0.00 \mathrm{E}+00$ & $0.00 E+00$ & $0.00 \mathrm{E}+00$ & $0.00 \mathrm{E}+00$ & $0.00 \mathrm{E}+00$ \\
\hline $\mathrm{S} 17$ & $0.00 \mathrm{E}+00$ & $0.00 \mathrm{E}+00$ & $0.00 \mathrm{E}+00$ & $0.00 \mathrm{E}+00$ & $0.00 \mathrm{E}+00$ & $0.00 \mathrm{E}+00$ & $0.00 \mathrm{E}+00$ & $0.00 \mathrm{E}+00$ & $0.00 \mathrm{E}+00$ & $0.00 \mathrm{E}+00$ & $0.00 \mathrm{E}+00$ & $0.00 \mathrm{E}+00$ & $0.00 \mathrm{E}+00$ & $0.00 \mathrm{E}+00$ & $0.00 \mathrm{E}+00$ & $0.00 \mathrm{E}+00$ \\
\hline $\mathrm{S} 18$ & 1.73E-06 & 6.27E-07 & $1.28 \mathrm{E}-08$ & $1.86 \mathrm{E}-08$ & 2.56E-07 & 6.44E-07 & 7.31E-04 & 4.40E-05 & $4.21 \mathrm{E}-05$ & 2.77E-04 & 1.15E-07 & 2.92E-05 & 1.11E-05 & $1.30 \mathrm{E}-03$ & 3.07E-06 & 6.47E-06 \\
\hline S19-O & $5.50 \mathrm{E}-08$ & 8.22E-08 & $2.28 \mathrm{E}-09$ & 1.91E-09 & $5.51 \mathrm{E}-08$ & $1.25 \mathrm{E}-06$ & $1.01 \mathrm{E}-04$ & $2.65 \mathrm{E}-06$ & $3.51 \mathrm{E}-06$ & 3.78E-05 & $2.18 E-08$ & 4.58E-06 & $6.50 \mathrm{E}-07$ & $1.30 \mathrm{E}-04$ & $9.50 E-07$ & 1.69E-06 \\
\hline S19-A & $6.27 \mathrm{E}-08$ & 6.37E-08 & 1.27E-09 & 1.14E-09 & 3.17E-08 & $1.68 \mathrm{E}-08$ & $2.52 \mathrm{E}-05$ & $9.71 \mathrm{E}-07$ & $1.43 E-06$ & 1.99E-05 & 2.47E-08 & $1.23 \mathrm{E}-06$ & 1.26E-06 & 3.33E-05 & $1.08 E-06$ & $1.92 E-06$ \\
\hline S20-T & $3.03 E-08$ & $3.08 E-08$ & $3.19 \mathrm{E}-11$ & $3.21 E-10$ & 7.26E-10 & 3.51E-09 & 1.30E-06 & $3.08 E-07$ & $1.13 E-06$ & $5.00 \mathrm{E}-07$ & $1.94 E-08$ & $1.71 E-07$ & 9.92E-07 & 1.39E-06 & 8.49E-07 & $1.51 E-06$ \\
\hline S20-B & 2.03E-07 & $2.06 E-07$ & 1.69E-09 & $2.15 E-09$ & $4.48 \mathrm{E}-08$ & 3.59E-08 & 9.71E-05 & 4.34E-06 & 7.52E-06 & 2.67E-05 & 1.31E-07 & 2.86E-06 & 6.62E-06 & 8.87E-05 & $5.27 E-06$ & $1.01 E-05$ \\
\hline S21 & $5.62 \mathrm{E}-08$ & 4.67E-08 & $1.58 \mathrm{E}-09$ & $1.68 \mathrm{E}-09$ & $3.85 \mathrm{E}-08$ & 3.51E-08 & 8.63E-05 & 4.37E-06 & 1.17E-05 & 2.79E-05 & $2.74 E-09$ & 3.57E-06 & $2.78 \mathrm{E}-06$ & $6.38 \mathrm{E}-04$ & $1.20 E-07$ & $2.58 \mathrm{E}-06$ \\
\hline S22 & $4.24 \mathrm{E}-08$ & $2.88 \mathrm{E}-08$ & $7.94 \mathrm{E}-10$ & $6.97 \mathrm{E}-10$ & 1.39E-08 & 4.79E-09 & $1.20 \mathrm{E}-04$ & 8.44E-06 & $6.38 \mathrm{E}-05$ & 2.47E-05 & 9.41E-09 & 4.62E-06 & 1.45E-05 & 2.48E-03 & $2.32 E-07$ & $1.38 \mathrm{E}-06$ \\
\hline & & & & & & & & & & & & & & & & \\
\hline & & & & & & & & & & & & & & & & \\
\hline & & & & & & & & & & & & & & & & \\
\hline & & & & & & & & & & & & & & & & \\
\hline & & & & & & & & & & & & & & & & \\
\hline & & & & & & & & & & & & & & & & \\
\hline & & & & & & & & & & & & & & & & \\
\hline & & & & & & & & & & & & & & & & \\
\hline & & & & & & & & & & & & & & & & \\
\hline & & & & & & & & & & & & & & & & \\
\hline
\end{tabular}




\begin{tabular}{|c|c|c|c|c|c|c|c|c|c|c|c|c|c|c|c|c|}
\hline & & & & & & & & & & & & & & & & \\
\hline & & & & & & & & & & & & & & & & \\
\hline & & & & & & & & & & & & & & & & \\
\hline & & & & & & & & & & & & & & & & \\
\hline & & & & & & & & & & & & & & & & \\
\hline Tank \# & $\begin{array}{c}0-233 \\
a\end{array}$ & U-234 & U-235 & U-236 & $0-238$ & Np-23r & $P u-238$ & Pu-239 & $P u-240$ & Pu-241 & $P u-242$ & Am-241 & Am-243 & $\mathrm{Cm}-244$ & $\mathrm{Cm}-245$ & $\mathrm{Cm}-246$ \\
\hline S1 & $\frac{y}{2.56 E-03}$ & $\frac{y}{4.03 E-03}$ & $\frac{\mathbf{g}}{4.92 \mathrm{~F}-02}$ & $\frac{g}{405 E-03}$ & $\frac{\mathrm{g}}{730 \mathrm{~F}+00}$ & $\frac{g}{404 E-03}$ & $\frac{\mathrm{g}}{275 \mathrm{~F}-04}$ & $\frac{9}{405 E_{-03}}$ & $\frac{9}{403 E_{-03}}$ & $\frac{9}{276 F_{-05}}$ & $\frac{\mathrm{g}}{105502}$ & $\frac{g}{56205}$ & $\frac{\mathrm{g}}{102502}$ & $\frac{\mathbf{g}}{2}$ & $\frac{\mathrm{g}}{2}$ & $\frac{\mathrm{g}}{4.06 \mathrm{~F}-03}$ \\
\hline S2 & $2.68 E-03$ & $423 F-03$ & $\begin{array}{l}4.92 \mathrm{E}-02 \\
3.40 \mathrm{~F}-01\end{array}$ & 4.05E-03 & 1.30E+U0 & 4.04E-US & 2.15E-U4 & 4.05E-US & 4.03E-U3 & $2.16 \mathrm{E}-05$ & 4.05E-03 & 5.69上-05 & 4.03E-03 & 6.69E-05 & 4.04E-03 & 4.06E-03 \\
\hline & & & $3.40 \mathrm{E}-01$ & $8.51 E-03$ & $5.45 \mathrm{E}+01$ & $7.75 E-02$ & 7.32E-04 & 9.04E-03 & $3.64 E-03$ & $2.90 E-05$ & $3.65 E-03$ & 6.67E-05 & 3.63E-03 & 3.19E-05 & $3.64 E-03$ & $3.66 E-03$ \\
\hline S3 & $6.63 E-03$ & $6.25 \mathrm{E}-02$ & $8.14 \mathrm{E}+00$ & 1.19E-01 & 1.07E+03 & 2.53E-01 & $5.20 \mathrm{E}-03$ & 3.69E-01 & $3.45 \mathrm{E}-02$ & $2.75 \mathrm{E}-04$ & $1.05 E-02$ & 8.50E-04 & 6.71E-03 & $2.82 \mathrm{E}-03$ & $1.05 E-02$ & $1.06 E-02$ \\
\hline S4 & $1.71 \mathrm{E}-03$ & 7.02E-03 & 7.05E-01 & 1.28E-02 & $9.26 \mathrm{E}+01$ & 2.07E-03 & 6.73E-04 & 1.84E-02 & 2.15E-03 & 3.68E-05 & $5.60 E-04$ & 2.65E-05 & 5.59E-04 & 1.93E-06 & 5.59E-04 & $5.64 E-04$ \\
\hline S5 & $1.10 E-04$ & $1.09 E-04$ & 1.92E-03 & $1.10 E-04$ & 2.95E-01 & $1.10 E-04$ & 3.67E-05 & $4.63 \mathrm{E}-04$ & 1.86E-04 & 3.12E-07 & $1.10 E-04$ & $3.30 \mathrm{E}-06$ & $3.17 \mathrm{E}-05$ & $1.10 E-04$ & $1.09 E-04$ & $1.10 E-04$ \\
\hline S6 & $9.35 \mathrm{E}-02$ & 4.79E-01 & $5.71 \mathrm{E}+01$ & $1.66 \mathrm{E}+00$ & $1.26 \mathrm{E}+04$ & $2.57 \mathrm{E}-01$ & 4.07E-02 & $1.75 \mathrm{E}+00$ & 7.66E-02 & 1.34E-03 & $6.60 \mathrm{E}-03$ & $3.41 \mathrm{E}-03$ & 5.84E-03 & 2.66E-04 & $2.60 \mathrm{E}-03$ & $3.26 \mathrm{E}-03$ \\
\hline S7 & $6.00 E-04$ & $6.14 \mathrm{E}-02$ & $5.56 \mathrm{E}+00$ & $2.06 \mathrm{E}-01$ & $1.29 \mathrm{E}+02$ & $1.52 \mathrm{E}-02$ & 8.99E-04 & 2.63E-01 & 1.66E-02 & $4.15 \mathrm{E}-04$ & 1.59E-03 & 7.37E-04 & $6.01 E-04$ & 2.33E-06 & $6.00 E-04$ & $6.02 E-04$ \\
\hline S8 & $1.01 \mathrm{E}-03$ & $2.27 \mathrm{E}-03$ & 6.76E-02 & 2.27E-03 & $1.58 \mathrm{E}+01$ & 1.34E-02 & 2.96E-03 & 1.10E-01 & 7.39E-03 & $6.71 \mathrm{E}-05$ & $2.82 E-04$ & $2.94 \mathrm{E}-04$ & $2.80 E-04$ & $6.55 \mathrm{E}-06$ & $2.81 E-04$ & $2.82 E-04$ \\
\hline S9 & $0.00 \mathrm{E}+00$ & $0.00 \mathrm{E}+00$ & $0.00 \mathrm{E}+00$ & $0.00 E+00$ & $0.00 \mathrm{E}+00$ & $0.00 \mathrm{E}+00$ & $0.00 \mathrm{E}+00$ & $0.00 \mathrm{E}+00$ & $0.00 \mathrm{E}+00$ & $0.00 \mathrm{E}+00$ & $0.00 \mathrm{E}+00$ & $0.00 \mathrm{E}+00$ & $0.00 E+00$ & $0.00 \mathrm{E}+00$ & $0.00 \mathrm{E}+00$ & $0.00 \mathrm{E}+00$ \\
\hline $\mathrm{S} 10$ & 1.51E-02 & $8.47 \mathrm{E}-03$ & 4.99E-01 & $2.43 \mathrm{E}-02$ & $6.43 E+01$ & 7.70E-02 & $3.60 \mathrm{E}-03$ & 5.97E-02 & $1.56 \mathrm{E}-02$ & $2.26 \mathrm{E}-04$ & $2.46 \mathrm{E}-03$ & 7.16E-04 & 4.69E-03 & 1.36E-03 & $1.51 \mathrm{E}-03$ & $1.79 \mathrm{E}-03$ \\
\hline S11 & 9.12E-06 & 9.11E-06 & 1.17E-04 & 9.14E-06 & 2.03E-02 & $9.14 E-06$ & $7.60 \mathrm{E}-06$ & 1.27E-04 & 1.88E-05 & 4.85E-07 & 9.16E-06 & $1.90 \mathrm{E}-06$ & 3.81E-06 & $3.82 \mathrm{E}-06$ & 9.12E-06 & $9.21 E-06$ \\
\hline $\mathrm{S} 12$ & $7.55 \mathrm{E}-03$ & 4.24E-03 & 2.49E-01 & 1.21E-02 & $3.22 \mathrm{E}+01$ & 3.85E-02 & $1.80 \mathrm{E}-03$ & $2.98 \mathrm{E}-02$ & 7.82E-03 & $1.13 \mathrm{E}-04$ & $1.23 \mathrm{E}-03$ & $3.58 \mathrm{E}-04$ & 2.34E-03 & $6.78 \mathrm{E}-04$ & $7.53 \mathrm{E}-04$ & 8.97E-04 \\
\hline $\mathrm{S} 13$ & 4.33E-02 & 2.67E-02 & $1.68 \mathrm{E}+00$ & 7.66E-02 & $2.75 \mathrm{E}+02$ & $3.70 \mathrm{E}-02$ & 1.44E-02 & 2.30E-01 & 2.37E-02 & 9.57E-04 & 5.11E-03 & $2.26 \mathrm{E}-03$ & 7.35E-04 & $2.59 E-05$ & 7.31E-04 & 7.39E-04 \\
\hline S14 & $6.70 E-04$ & $1.05 E-03$ & $2.54 \mathrm{E}-02$ & $1.05 E-03$ & $4.07 \mathrm{E}+00$ & $1.72 \mathrm{E}-03$ & 4.09E-03 & 7.72E-03 & 4.95E-03 & 6.77E-07 & $1.06 E-03$ & $1.45 \mathrm{E}-03$ & 1.92E-03 & 4.70E-04 & $1.05 E-03$ & $1.06 E-03$ \\
\hline $\mathrm{S} 15$ & $0.00 \mathrm{E}+00$ & $0.00 \mathrm{E}+00$ & $0.00 \mathrm{E}+00$ & $0.00 \mathrm{E}+00$ & $0.00 \mathrm{E}+00$ & $0.00 E+00$ & $0.00 \mathrm{E}+00$ & $0.00 \mathrm{E}+00$ & $0.00 E+00$ & $0.00 \mathrm{E}+00$ & $0.00 \mathrm{E}+00$ & $0.00 \mathrm{E}+00$ & $0.00 \mathrm{E}+00$ & $0.00 \mathrm{E}+00$ & $0.00 \mathrm{E}+00$ & $0.00 \mathrm{E}+00$ \\
\hline $\mathrm{S} 16$ & $0.00 \mathrm{E}+00$ & $0.00 \mathrm{E}+00$ & $0.00 \mathrm{E}+00$ & $0.00 \mathrm{E}+00$ & $0.00 \mathrm{E}+00$ & $0.00 \mathrm{E}+00$ & $0.00 \mathrm{E}+00$ & $0.00 \mathrm{E}+00$ & $0.00 \mathrm{E}+00$ & $0.00 \mathrm{E}+00$ & $0.00 E+00$ & $0.00 \mathrm{E}+00$ & $0.00 \mathrm{E}+00$ & $0.00 \mathrm{E}+00$ & $0.00 \mathrm{E}+00$ & $0.00 \mathrm{E}+00$ \\
\hline S17 & $0.00 \mathrm{E}+00$ & $0.00 \mathrm{E}+00$ & $0.00 \mathrm{E}+00$ & $0.00 \mathrm{E}+00$ & $0.00 \mathrm{E}+00$ & $0.00 \mathrm{E}+00$ & $0.00 \mathrm{E}+00$ & $0.00 \mathrm{E}+00$ & $0.00 \mathrm{E}+00$ & $0.00 \mathrm{E}+00$ & $0.00 \mathrm{E}+00$ & $0.00 \mathrm{E}+00$ & $0.00 \mathrm{E}+00$ & $0.00 \mathrm{E}+00$ & $0.00 \mathrm{E}+00$ & $0.00 \mathrm{E}+00$ \\
\hline S18 & $7.55 \mathrm{E}-03$ & 4.24E-03 & $2.49 \mathrm{E}-01$ & 1.21E-02 & $3.22 \mathrm{E}+01$ & $3.85 \mathrm{E}-02$ & $1.80 \mathrm{E}-03$ & $2.98 \mathrm{E}-02$ & 7.82E-03 & $1.13 \mathrm{E}-04$ & 1.23E-03 & $3.58 \mathrm{E}-04$ & 2.34E-03 & $6.78 \mathrm{E}-04$ & $7.53 \mathrm{E}-04$ & $8.97 \mathrm{E}-04$ \\
\hline S19-O & 1.63E-02 & $3.75 \mathrm{E}-02$ & $3.01 \mathrm{E}+00$ & 8.41E-02 & $4.67 \mathrm{E}+02$ & $5.06 \mathrm{E}+00$ & 1.68E-02 & 1.22E-01 & 4.40E-02 & 1.04E-03 & $1.58 E-02$ & $3.80 \mathrm{E}-03$ & $9.26 \mathrm{E}-03$ & 4.57E-03 & 1.57E-02 & $1.58 E-02$ \\
\hline S19-A & 1.70E-02 & $2.67 \mathrm{E}-02$ & $1.54 \mathrm{E}+00$ & 4.61E-02 & $2.47 \mathrm{E}+02$ & $6.24 \mathrm{E}-02$ & $3.86 \mathrm{E}-03$ & $4.10 \mathrm{E}-02$ & $1.65 E-02$ & $5.03 \mathrm{E}-04$ & $1.65 E-02$ & $9.38 \mathrm{E}-04$ & $1.65 E-02$ & $1.08 \mathrm{E}-03$ & $1.64 E-02$ & $1.65 E-02$ \\
\hline S20-T & 3.50E-02 & $5.49 E-02$ & $1.64 \mathrm{E}-01$ & 5.52E-02 & $2.40 \mathrm{E}+01$ & $5.54 E-02$ & 8.42E-04 & $5.51 E-02$ & $5.54 E-02$ & 5.38E-05 & $5.49 E-02$ & $5.53 E-04$ & $5.52 E-02$ & $1.90 \mathrm{E}-04$ & $5.49 E-02$ & $5.52 E-02$ \\
\hline S20-B & 3.34E-02 & $5.24 E-02$ & $1.24 \mathrm{E}+00$ & $5.28 E-02$ & $2.12 \mathrm{E}+02$ & 8.08E-02 & 9.02E-03 & 1.11E-01 & $5.26 E-02$ & $4.10 \mathrm{E}-04$ & $5.29 E-02$ & 1.32E-03 & $5.25 E-02$ & 1.74E-03 & $4.86 E-02$ & $5.27 E-02$ \\
\hline S21 & 4.14E-02 & 5.31E-02 & $5.21 \mathrm{E}+00$ & $1.84 \mathrm{E}-01$ & $8.14 \mathrm{E}+02$ & $3.53 \mathrm{E}-01$ & $3.58 \mathrm{E}-02$ & 4.99E-01 & 3.67E-01 & 1.91E-03 & $4.95 E-03$ & 7.37E-03 & $9.88 \mathrm{E}-02$ & $5.59 \mathrm{E}-02$ & $4.95 E-03$ & $6.02 \mathrm{E}-02$ \\
\hline $\mathrm{S} 22$ & 2.38E-03 & $2.50 \mathrm{E}-03$ & 1.99E-01 & 5.84E-03 & $2.24 E+01$ & 3.68E-03 & 3.79E-03 & 7.36E-02 & 1.52E-01 & 1.29E-04 & 1.30E-03 & $7.28 \mathrm{E}-04$ & 3.93E-02 & 1.66E-02 & 7.31E-04 & $2.46 \mathrm{E}-03$ \\
\hline & & & & & & & & & & & & & & & & \\
\hline & & & & & & & & & & & & & & & & \\
\hline & & & & & & & & & & & & & & & & \\
\hline & & & & & & & & & & & & & & & & \\
\hline & & & & & & & & & & & & & & & & \\
\hline & & & & & & & & & & & & & & & & \\
\hline & & & & & & & & & & & & & & & & \\
\hline & & & & & & & & & & & & & & & & \\
\hline & & & & & & & & & & & & & & & & \\
\hline & & & & & & & & & & & & & & & & \\
\hline
\end{tabular}




\begin{tabular}{|c|c|c|c|c|c|c|c|c|c|c|c|c|c|c|}
\hline & & & & \\
\hline & & & & & & & & & & & & & & \\
\hline & & & & & & & & & & & & & & \\
\hline & & & & & & & & & & & & & & \\
\hline \multirow[b]{2}{*}{ Tank \# } & U-233 & U-234 & U-235 & U-236 & U-238 & Np-237 & Pu-238 & Pu-239 & Pu-240 & Pu-241 & Pu-242 & Am-241 & Am-243 & $\mathrm{Cm}-244$ \\
\hline & g/(g waste) & g/(g waste) & g/(g waste) & $g /(g$ waste) & g/(g waste) & g/(g waste) & g/(g waste) & $\mathrm{g} /$ (g waste) & g/(g waste) & g/(g waste) & $\mathrm{g} /$ (g waste) & $\mathrm{g} /(\mathrm{g}$ waste) & g/(g waste) & $\mathrm{g} /(\mathrm{g}$ waste $)$ \\
\hline S1 & $6.59 E-05$ & $1.04 E-04$ & $1.27 \mathrm{E}-03$ & $1.04 E-04$ & $1.88 \mathrm{E}-01$ & $1.04 E-04$ & $7.09 \mathrm{E}-06$ & $1.05 E-04$ & $1.04 E-04$ & $7.11 \mathrm{E}-07$ & $1.04 \mathrm{E}-04$ & $1.47 \mathrm{E}-06$ & $1.04 E-04$ & $1.72 \mathrm{E}-06$ \\
\hline S2 & $3.16 E-05$ & $4.98 E-05$ & $4.00 \mathrm{E}-03$ & $1.00 E-04$ & $6.43 \mathrm{E}-01$ & $1.35 E-04$ & 8.63E-06 & 1.07E-04 & $4.29 E-05$ & $3.41 E-07$ & 4.30E-05 & 7.86E-07 & $4.28 E-05$ & 3.76E-07 \\
\hline S3 & 3.02E-06 & $2.85 E-05$ & 3.70E-03 & 5.42E-05 & 4.86E-01 & $1.15 \mathrm{E}-04$ & $2.36 \mathrm{E}-06$ & 1.68E-04 & 1.57E-05 & $1.25 \mathrm{E}-07$ & 4.76E-06 & 3.87E-07 & $3.06 \mathrm{E}-06$ & $1.28 \mathrm{E}-06$ \\
\hline S4 & 5.66E-06 & 2.33E-05 & 2.34E-03 & 4.23E-05 & 3.07E-01 & 6.86E-06 & 2.23E-06 & 6.09E-05 & 7.13E-06 & 1.22E-07 & $1.86 E-06$ & 8.79E-08 & $1.86 E-06$ & 6.38E-09 \\
\hline S5 & $3.15 E-05$ & $3.14 E-05$ & 5.53E-04 & $3.15 E-05$ & 8.46E-02 & $3.15 E-05$ & 1.06E-05 & 1.33E-04 & 5.34E-05 & 8.97E-08 & $3.16 E-05$ & $9.49 \mathrm{E}-07$ & $9.10 \mathrm{E}-06$ & $3.15 E-05$ \\
\hline S6 & $5.66 \mathrm{E}-06$ & 2.91E-05 & 3.46E-03 & 1.00E-04 & 7.63E-01 & $1.56 \mathrm{E}-05$ & $2.46 \mathrm{E}-06$ & 1.06E-04 & 4.64E-06 & $8.14 \mathrm{E}-08$ & 4.00E-07 & 2.07E-07 & $3.54 \mathrm{E}-07$ & $1.61 \mathrm{E}-08$ \\
\hline S7 & $2.41 E-07$ & 2.46E-05 & 2.23E-03 & 8.27E-05 & 5.18E-02 & 6.11E-06 & 3.60E-07 & 1.05E-04 & 6.66E-06 & 1.67E-07 & 6.38E-07 & 2.95E-07 & $2.41 E-07$ & $9.35 E-10$ \\
\hline S8 & $2.05 E-06$ & 4.63E-06 & $1.38 \mathrm{E}-04$ & 4.64E-06 & 3.22E-02 & $2.74 \mathrm{E}-05$ & 6.04E-06 & 2.24E-04 & $1.51 \mathrm{E}-05$ & 1.37E-07 & $5.75 E-07$ & 6.00E-07 & 5.70E-07 & 1.33E-08 \\
\hline S9 & $0.00 \mathrm{E}+00$ & $0.00 \mathrm{E}+00$ & $0.00 \mathrm{E}+00$ & $0.00 \mathrm{E}+00$ & $0.00 \mathrm{E}+00$ & $0.00 \mathrm{E}+00$ & $0.00 \mathrm{E}+00$ & $0.00 \mathrm{E}+00$ & $0.00 \mathrm{E}+00$ & $0.00 \mathrm{E}+00$ & $0.00 \mathrm{E}+00$ & $0.00 \mathrm{E}+00$ & $0.00 \mathrm{E}+00$ & $0.00 \mathrm{E}+00$ \\
\hline S10 & $1.79 \mathrm{E}-04$ & $1.00 \mathrm{E}-04$ & 5.91E-03 & 2.88E-04 & 7.63E-01 & $9.13 \mathrm{E}-04$ & 4.27E-05 & 7.08E-04 & $1.85 \mathrm{E}-04$ & $2.68 \mathrm{E}-06$ & $2.92 \mathrm{E}-05$ & 8.49E-06 & 5.56E-05 & $1.61 \mathrm{E}-05$ \\
\hline S11 & 2.41E-06 & 2.40E-06 & $3.08 \mathrm{E}-05$ & 2.41E-06 & 5.36E-03 & 2.41E-06 & $2.00 \mathrm{E}-06$ & 3.36E-05 & 4.97E-06 & $1.28 \mathrm{E}-07$ & $2.42 E-06$ & $5.00 \mathrm{E}-07$ & $1.00 \mathrm{E}-06$ & $1.01 \mathrm{E}-06$ \\
\hline S12 & $1.79 \mathrm{E}-04$ & $1.00 \mathrm{E}-04$ & $5.91 \mathrm{E}-03$ & $2.88 \mathrm{E}-04$ & $7.63 \mathrm{E}-01$ & $9.13 \mathrm{E}-04$ & 4.27E-05 & 7.08E-04 & $1.85 \mathrm{E}-04$ & $2.68 \mathrm{E}-06$ & $2.92 E-05$ & $8.49 \mathrm{E}-06$ & 5.56E-05 & $1.61 \mathrm{E}-05$ \\
\hline S13 & $2.39 \mathrm{E}-04$ & 1.47E-04 & $9.28 \mathrm{E}-03$ & $4.23 \mathrm{E}-04$ & $1.52 \mathrm{E}+00$ & $2.04 \mathrm{E}-04$ & 7.93E-05 & $1.27 \mathrm{E}-03$ & $1.31 \mathrm{E}-04$ & $5.28 \mathrm{E}-06$ & $2.82 \mathrm{E}-05$ & $1.25 \mathrm{E}-05$ & $4.06 E-06$ & $1.43 E-07$ \\
\hline S14 & $2.63 E-06$ & 4.13E-06 & $1.00 \mathrm{E}-04$ & 4.14E-06 & $1.60 \mathrm{E}-02$ & $6.77 \mathrm{E}-06$ & $1.61 \mathrm{E}-05$ & $3.03 \mathrm{E}-05$ & $1.95 \mathrm{E}-05$ & $2.66 E-09$ & $4.15 E-06$ & $5.69 \mathrm{E}-06$ & 7.56E-06 & $1.85 \mathrm{E}-06$ \\
\hline $\mathrm{S} 15$ & $0.00 E+00$ & $0.00 E+00$ & $0.00 E+00$ & $0.00 E+00$ & $0.00 E+00$ & $0.00 E+00$ & $0.00 E+00$ & $0.00 E+00$ & $0.00 E+00$ & $0.00 E+00$ & $0.00 E+00$ & $0.00 E+00$ & $0.00 E+00$ & $0.00 E+00$ \\
\hline S16 & $0.00 E+00$ & $0.00 E+00$ & $0.00 \mathrm{E}+00$ & $0.00 E+00$ & $0.00 \mathrm{E}+00$ & $0.00 \mathrm{E}+00$ & $0.00 \mathrm{E}+00$ & $0.00 E+00$ & $0.00 E+00$ & $0.00 E+00$ & $0.00 \mathrm{E}+00$ & $0.00 E+00$ & $0.00 E+00$ & $0.00 \mathrm{E}+00$ \\
\hline $\mathrm{S} 17$ & $0.00 E+00$ & $0.00 E+00$ & $0.00 \mathrm{E}+00$ & $0.00 E+00$ & $0.00 \mathrm{E}+00$ & $0.00 \mathrm{E}+00$ & $0.00 \mathrm{E}+00$ & $0.00 E+00$ & $0.00 E+00$ & $0.00 E+00$ & $0.00 \mathrm{E}+00$ & $0.00 \mathrm{E}+00$ & $0.00 E+00$ & $0.00 \mathrm{E}+00$ \\
\hline $\mathrm{S} 18$ & $1.79 \mathrm{E}-04$ & $1.00 \mathrm{E}-04$ & $5.91 \mathrm{E}-03$ & $2.88 \mathrm{E}-04$ & 7.63E-01 & $9.13 \mathrm{E}-04$ & 4.27E-05 & 7.08E-04 & $1.85 \mathrm{E}-04$ & 2.68E-06 & 2.92E-05 & 8.49E-06 & 5.56E-05 & $1.61 \mathrm{E}-05$ \\
\hline S19-O & $5.71 \mathrm{E}-06$ & 1.32E-05 & $1.06 \mathrm{E}-03$ & $2.95 \mathrm{E}-05$ & $1.64 \mathrm{E}-01$ & $1.78 \mathrm{E}-03$ & 5.91E-06 & $4.27 \mathrm{E}-05$ & $1.54 \mathrm{E}-05$ & $3.65 \mathrm{E}-07$ & $5.55 E-06$ & 1.33E-06 & $3.25 \mathrm{E}-06$ & $1.60 \mathrm{E}-06$ \\
\hline S19-A & $6.50 \mathrm{E}-06$ & $1.02 \mathrm{E}-05$ & 5.89E-04 & 1.76E-05 & $9.43 \mathrm{E}-02$ & $2.38 \mathrm{E}-05$ & $1.47 \mathrm{E}-06$ & 1.56E-05 & $6.30 E-06$ & 1.92E-07 & $6.28 E-06$ & $3.58 \mathrm{E}-07$ & $6.30 E-06$ & $4.11 \mathrm{E}-07$ \\
\hline S20-T & $3.14 E-06$ & $4.94 E-06$ & $1.48 \mathrm{E}-05$ & $4.96 E-06$ & $2.16 \mathrm{E}-03$ & $4.98 E-06$ & 7.57E-08 & $4.96 E-06$ & $4.98 E-06$ & 4.84E-09 & $4.94 E-06$ & 4.97E-08 & $4.96 E-06$ & $1.71 \mathrm{E}-08$ \\
\hline S20-B & $2.11 E-05$ & $3.30 E-05$ & 7.83E-04 & $3.32 E-05$ & $1.33 \mathrm{E}-01$ & $5.09 \mathrm{E}-05$ & $5.68 \mathrm{E}-06$ & 6.99E-05 & $3.31 E-05$ & $2.58 \mathrm{E}-07$ & $3.33 E-05$ & 8.32E-07 & $3.31 E-05$ & $1.09 \mathrm{E}-06$ \\
\hline S21 & $5.83 \mathrm{E}-06$ & $7.48 \mathrm{E}-06$ & 7.33E-04 & $2.60 \mathrm{E}-05$ & $1.15 \mathrm{E}-01$ & 4.97E-05 & $5.05 E-06$ & 7.03E-05 & $5.16 \mathrm{E}-05$ & $2.70 \mathrm{E}-07$ & 6.97E-07 & $1.04 \mathrm{E}-06$ & $1.39 \mathrm{E}-05$ & $7.88 \mathrm{E}-06$ \\
\hline S22 & 4.39E-06 & 4.62E-06 & 3.68E-04 & $1.08 \mathrm{E}-05$ & 4.14E-02 & $6.79 \mathrm{E}-06$ & 7.00E-06 & $1.36 \mathrm{E}-04$ & 2.81E-04 & 2.39E-07 & $2.39 \mathrm{E}-06$ & $1.34 \mathrm{E}-06$ & 7.26E-05 & 3.06E-05 \\
\hline & & & & & & & & & & & & & & \\
\hline & & & & & & & & & & & & & & \\
\hline & & & & & & & & & & & & & & \\
\hline & & & & & & & & & & & & & & \\
\hline & & & & & & & & & & & & & & \\
\hline & & & & & & & & & & & & & & \\
\hline & & & & & & & & & & & & & & \\
\hline & & & & & & & & & & & & & & \\
\hline & & & & & & & & & & & & & & \\
\hline & & & & & & & & & & & & & & \\
\hline
\end{tabular}


Appendix 3

WSRC-TR-2000-00011

Revision 0

January 21, 2000

Page 40

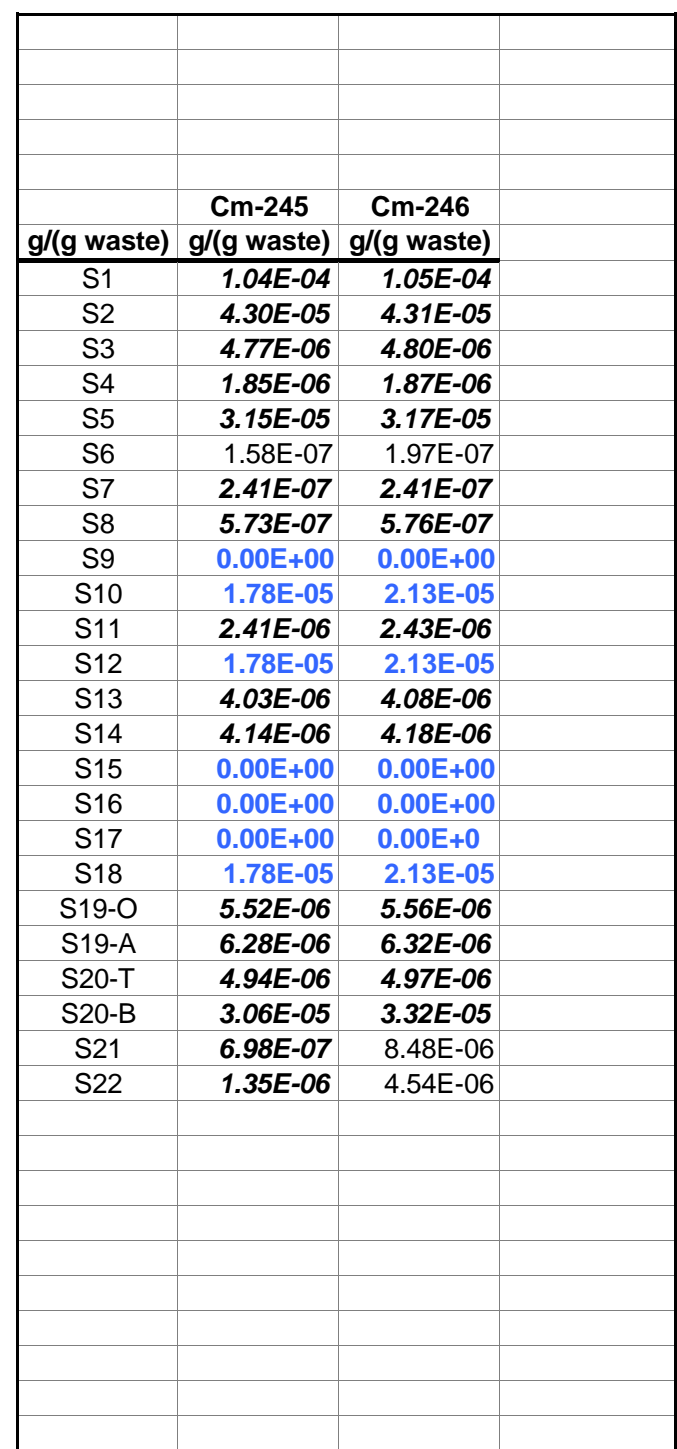




\begin{tabular}{|c|c|c|c|c|c|c|c|c|c|c|c|c|c|c|c|c|}
\hline \multicolumn{17}{|c|}{ Solvent Tank Nuclide Inventory Calculations - Sludge } \\
\hline & $\mathrm{Ci} / \mathbf{q}$ & & $\mathrm{Ci} / \mathbf{q}$ & & $\mathrm{Ci} / \mathrm{q}$ & & $\mathrm{Ci} / \mathbf{q}$ & & $\mathrm{Ci} / \mathbf{q}$ & & $\mathrm{Ci} / \mathbf{q}$ & & $\mathrm{Ci} / \mathbf{q}$ & & $\mathrm{Ci} / \mathrm{q}$ & \\
\hline \multirow[t]{2}{*}{ SPCAT } & 9.64E-03 & & $6.24 \mathrm{E}-03$ & & 2.16E-06 & & 6.47E-05 & & 3.36E-07 & & 7.05E-04 & & $1.71 \mathrm{E}+01$ & & 6.21E-02 & \\
\hline & U-233 & Error & U-234 & Error & U-235 & Error & U-236 & Error & U-238 & Error & Np-237 & Error & Pu-238 & Error & Pu-239 & Error \\
\hline Tank \# & $\mathrm{pCi} / \mathrm{g}$ & $\mathrm{pCi} / \mathrm{g}$ & $\mathrm{pCi} / \mathrm{g}$ & $\mathrm{pCi} / \mathrm{g}$ & $\mathrm{pCi} / \mathrm{g}$ & $\mathrm{pCi} / \mathrm{g}$ & $\mathrm{pCi} / \mathrm{g}$ & $\mathrm{pCi} / \mathrm{g}$ & $\mathrm{pCi} / \mathrm{g}$ & $\mathrm{pCi} / \mathrm{g}$ & $\mathrm{pCi} / \mathrm{g}$ & $\mathrm{pCi} / \mathrm{g}$ & $\mathrm{pCi} / \mathrm{g}$ & $\mathrm{pCi} / \mathrm{g}$ & $\mathrm{pCi} / \mathrm{g}$ & $\mathrm{pCi} / \mathrm{g}$ \\
\hline $\mathrm{S} 1$ & $2.06 \mathrm{E}+05$ & $4.12 E+04$ & $5.02 E+04$ & $1.43 E+05$ & $6.19 E+02$ & $1.96 \mathrm{E}+03$ & $1.08 E+03$ & $2.30 \mathrm{E}+03$ & $1.90 \mathrm{E}+04$ & $4.00 E+04$ & $2.38 E+03$ & $4.46 \mathrm{E}+03$ & $1.36 \mathrm{E}+07$ & $4.38 \mathrm{E}+07$ & $4.69 E+05$ & $7.40 E+05$ \\
\hline S2 & $5.90 E+03$ & $0.00 E+00$ & $1.01 E+04$ & $0.00 E+00$ & $3.46 \mathrm{E}+01$ & $6.92 \mathrm{E}+00$ & $3.97 E+01$ & $0.00 E+00$ & $8.24 \mathrm{E}+02$ & $1.65 \mathrm{E}+02$ & $1.07 E+03$ & $0.00 E+00$ & $5.95 \mathrm{E}+07$ & $2.38 \mathrm{E}+07$ & $3.05 E+05$ & $0.00 E+00$ \\
\hline S3 & $2.06 \mathrm{E}+05$ & $4.12 \mathrm{E}+04$ & $5.02 \mathrm{E}+04$ & $1.43 \mathrm{E}+05$ & $6.19 \mathrm{E}+02$ & $1.96 \mathrm{E}+03$ & $1.08 \mathrm{E}+03$ & $2.30 \mathrm{E}+03$ & $1.90 \mathrm{E}+04$ & $4.00 \mathrm{E}+04$ & $2.38 \mathrm{E}+03$ & $4.46 \mathrm{E}+03$ & $1.36 \mathrm{E}+07$ & $4.38 \mathrm{E}+07$ & $4.69 \mathrm{E}+05$ & $7.40 \mathrm{E}+05$ \\
\hline S4(1) & $0.00 \mathrm{E}+00$ & $0.00 \mathrm{E}+00$ & $9.23 E+06$ & $5.54 E+06$ & $3.57 E+05$ & $0.00 E+00$ & $1.08 \mathrm{E}+03$ & $2.30 \mathrm{E}+03$ & $9.23 \mathrm{E}+06$ & $5.54 \mathrm{E}+06$ & $3.29 E+05$ & $0.00 E+00$ & $8.20 \mathrm{E}+07$ & $3.48 \mathrm{E}+07$ & $3.96 E+06$ & $2.38 E+06$ \\
\hline S5 & $2.06 \mathrm{E}+05$ & $4.12 \mathrm{E}+04$ & $5.02 \mathrm{E}+04$ & $1.43 \mathrm{E}+05$ & $6.19 \mathrm{E}+02$ & $1.96 \mathrm{E}+03$ & $1.08 \mathrm{E}+03$ & $2.30 \mathrm{E}+03$ & $1.90 \mathrm{E}+04$ & $4.00 \mathrm{E}+04$ & $2.38 \mathrm{E}+03$ & $4.46 \mathrm{E}+03$ & $1.36 \mathrm{E}+07$ & $4.38 \mathrm{E}+07$ & $4.69 \mathrm{E}+05$ & $7.40 \mathrm{E}+05$ \\
\hline S6 & $2.06 \mathrm{E}+05$ & $4.12 \mathrm{E}+04$ & $5.02 \mathrm{E}+04$ & $1.43 E+05$ & $6.19 \mathrm{E}+02$ & $1.96 \mathrm{E}+03$ & $1.08 \mathrm{E}+03$ & $2.30 \mathrm{E}+03$ & $1.90 \mathrm{E}+04$ & $4.00 \mathrm{E}+04$ & $2.38 \mathrm{E}+03$ & $4.46 \mathrm{E}+03$ & $1.36 \mathrm{E}+07$ & $4.38 \mathrm{E}+07$ & $4.69 \mathrm{E}+05$ & $7.40 \mathrm{E}+05$ \\
\hline S7 & $1.13 E+04$ & $0.00 E+00$ & $1.72 \mathrm{E}+04$ & $3.43 E+03$ & $5.81 \mathrm{E}+02$ & $1.16 \mathrm{E}+02$ & $6.35 \mathrm{E}+02$ & $1.27 \mathrm{E}+02$ & $1.72 \mathrm{E}+04$ & $3.44 \mathrm{E}+03$ & $8.24 E+02$ & $0.00 E+00$ & $2.02 \mathrm{E}+05$ & $8.07 \mathrm{E}+04$ & $4.05 E+05$ & $8.09 E+04$ \\
\hline S8 & $1.92 E+03$ & $0.00 E+00$ & $1.24 E+03$ & $0.00 E+00$ & $7.30 \mathrm{E}+00$ & $1.46 \mathrm{E}+00$ & $1.29 E+01$ & $0.00 E+00$ & $1.66 \mathrm{E}+02$ & $3.32 \mathrm{E}+01$ & $2.09 E+02$ & $0.00 E+00$ & $3.81 E+05$ & $0.00 E+00$ & $3.66 \mathrm{E}+04$ & $7.32 \mathrm{E}+03$ \\
\hline S9 & $2.06 \mathrm{E}+05$ & $4.12 \mathrm{E}+04$ & $5.02 \mathrm{E}+04$ & $1.43 E+05$ & $6.19 \mathrm{E}+02$ & $1.96 \mathrm{E}+03$ & $1.08 \mathrm{E}+03$ & $2.30 \mathrm{E}+03$ & $1.90 \mathrm{E}+04$ & $4.00 \mathrm{E}+04$ & $2.38 \mathrm{E}+03$ & $4.46 \mathrm{E}+03$ & $1.36 \mathrm{E}+07$ & $4.38 \mathrm{E}+07$ & $4.69 \mathrm{E}+05$ & $7.40 \mathrm{E}+05$ \\
\hline $\mathrm{S} 10$ & $2.06 \mathrm{E}+05$ & $4.12 \mathrm{E}+04$ & $5.02 \mathrm{E}+04$ & $1.43 E+05$ & $6.19 \mathrm{E}+02$ & $1.96 \mathrm{E}+03$ & $1.08 \mathrm{E}+03$ & $2.30 \mathrm{E}+03$ & $1.90 \mathrm{E}+04$ & $4.00 \mathrm{E}+04$ & $2.38 E+03$ & $4.46 \mathrm{E}+03$ & $1.36 \mathrm{E}+07$ & $4.38 \mathrm{E}+07$ & $4.69 \mathrm{E}+05$ & $7.40 \mathrm{E}+05$ \\
\hline S11 & $2.06 \mathrm{E}+05$ & $4.12 \mathrm{E}+04$ & $5.02 \mathrm{E}+04$ & $1.43 E+05$ & $6.19 \mathrm{E}+02$ & $1.96 \mathrm{E}+03$ & $1.08 E+03$ & $2.30 \mathrm{E}+03$ & $1.90 \mathrm{E}+04$ & $4.00 \mathrm{E}+04$ & $2.38 \mathrm{E}+03$ & $4.46 \mathrm{E}+03$ & $1.36 \mathrm{E}+07$ & $4.38 \mathrm{E}+07$ & $4.69 \mathrm{E}+05$ & $7.40 \mathrm{E}+05$ \\
\hline $\mathrm{S} 12$ & $2.06 \mathrm{E}+05$ & $4.12 \mathrm{E}+04$ & $5.02 \mathrm{E}+04$ & $1.43 E+05$ & $6.19 \mathrm{E}+02$ & $1.96 \mathrm{E}+03$ & $1.08 \mathrm{E}+03$ & $2.30 \mathrm{E}+03$ & $1.90 \mathrm{E}+04$ & $4.00 \mathrm{E}+04$ & $2.38 \mathrm{E}+03$ & $4.46 \mathrm{E}+03$ & $1.36 \mathrm{E}+07$ & $4.38 \mathrm{E}+07$ & $4.69 \mathrm{E}+05$ & $7.40 \mathrm{E}+05$ \\
\hline $\mathrm{S} 13$ & $2.06 \mathrm{E}+05$ & $4.12 \mathrm{E}+04$ & $5.02 \mathrm{E}+04$ & $1.43 E+05$ & $6.19 \mathrm{E}+02$ & $1.96 \mathrm{E}+03$ & $1.08 \mathrm{E}+03$ & $2.30 \mathrm{E}+03$ & $1.90 \mathrm{E}+04$ & $4.00 \mathrm{E}+04$ & $2.38 E+03$ & $4.46 \mathrm{E}+03$ & $1.36 \mathrm{E}+07$ & $4.38 \mathrm{E}+07$ & $4.69 \mathrm{E}+05$ & $7.40 \mathrm{E}+05$ \\
\hline S14 & $2.06 \mathrm{E}+05$ & $4.12 \mathrm{E}+04$ & $5.02 \mathrm{E}+04$ & $1.43 E+05$ & $6.19 \mathrm{E}+02$ & $1.96 \mathrm{E}+03$ & $1.08 \mathrm{E}+03$ & $2.30 \mathrm{E}+03$ & $1.90 \mathrm{E}+04$ & $4.00 \mathrm{E}+04$ & $2.38 \mathrm{E}+03$ & $4.46 \mathrm{E}+03$ & $1.36 \mathrm{E}+07$ & $4.38 \mathrm{E}+07$ & $4.69 \mathrm{E}+05$ & $7.40 \mathrm{E}+05$ \\
\hline S15 & $2.06 \mathrm{E}+05$ & $4.12 \mathrm{E}+04$ & $5.02 \mathrm{E}+04$ & $1.43 E+05$ & $6.19 \mathrm{E}+02$ & $1.96 \mathrm{E}+03$ & $1.08 \mathrm{E}+03$ & $2.30 \mathrm{E}+03$ & $1.90 \mathrm{E}+04$ & $4.00 \mathrm{E}+04$ & $2.38 \mathrm{E}+03$ & $4.46 \mathrm{E}+03$ & $1.36 \mathrm{E}+07$ & $4.38 \mathrm{E}+07$ & $4.69 \mathrm{E}+05$ & $7.40 \mathrm{E}+05$ \\
\hline S16 & $6.31 E+04$ & $0.00 E+00$ & $4.08 E+04$ & $0.00 E+00$ & 8.87E+02 & 1.77E+02 & $9.23 \mathrm{E}+02$ & $1.85 \mathrm{E}+02$ & $2.54 \mathrm{E}+04$ & $5.08 \mathrm{E}+03$ & $3.15 E+03$ & $0.00 E+00$ & $7.39 E+05$ & $2.95 \mathrm{E}+05$ & $7.16 \mathrm{E}+05$ & $1.43 \mathrm{E}+05$ \\
\hline $\mathrm{S} 17$ & $2.06 \mathrm{E}+05$ & $4.12 \mathrm{E}+04$ & $5.02 \mathrm{E}+04$ & $1.43 E+05$ & $6.19 \mathrm{E}+02$ & $1.96 \mathrm{E}+03$ & $1.08 \mathrm{E}+03$ & $2.30 \mathrm{E}+03$ & $1.90 \mathrm{E}+04$ & $4.00 \mathrm{E}+04$ & $2.38 \mathrm{E}+03$ & $4.46 \mathrm{E}+03$ & $1.36 \mathrm{E}+07$ & $4.38 \mathrm{E}+07$ & $4.69 \mathrm{E}+05$ & $7.40 \mathrm{E}+05$ \\
\hline S18 & $2.06 \mathrm{E}+05$ & $4.12 \mathrm{E}+04$ & $5.02 \mathrm{E}+04$ & $1.43 \mathrm{E}+05$ & $6.19 \mathrm{E}+02$ & $1.96 \mathrm{E}+03$ & $1.08 \mathrm{E}+03$ & $2.30 \mathrm{E}+03$ & $1.90 \mathrm{E}+04$ & $4.00 \mathrm{E}+04$ & $2.38 E+03$ & $4.46 \mathrm{E}+03$ & $1.36 \mathrm{E}+07$ & $4.38 \mathrm{E}+07$ & $4.69 \mathrm{E}+05$ & $7.40 \mathrm{E}+05$ \\
\hline S19 & $2.06 \mathrm{E}+05$ & $4.12 \mathrm{E}+04$ & $1.32 \mathrm{E}+05$ & $2.63 E+04$ & $2.90 \mathrm{E}+03$ & $5.80 \mathrm{E}+02$ & $3.04 \mathrm{E}+03$ & $6.08 \mathrm{E}+02$ & $4.13 \mathrm{E}+04$ & $8.25 E+03$ & $4.77 \mathrm{E}+03$ & $9.55 E+02$ & $2.40 \mathrm{E}+07$ & $9.60 \mathrm{E}+06$ & $9.68 \mathrm{E}+05$ & $1.94 \mathrm{E}+05$ \\
\hline $\mathrm{S} 20$ & $1.80 E+04$ & $0.00 E+00$ & $1.16 E+04$ & $0.00 E+00$ & $5.00 \mathrm{E}+02$ & $1.00 \mathrm{E}+02$ & $7.84 \mathrm{E}+02$ & $1.57 \mathrm{E}+02$ & $4.77 \mathrm{E}+04$ & $9.55 E+03$ & $2.02 \mathrm{E}+03$ & $4.04 \mathrm{E}+02$ & $5.77 \mathrm{E}+06$ & $2.31 \mathrm{E}+06$ & $8.15 \mathrm{E}+05$ & $1.63 \mathrm{E}+05$ \\
\hline $\mathrm{S} 21$ & $3.25 E+03$ & $0.00 E+00$ & $2.09 E+03$ & $0.00 E+00$ & $1.23 \mathrm{E}+01$ & $4.94 \mathrm{E}+00$ & $2.18 E+01$ & $0.00 E+00$ & $3.38 \mathrm{E}+02$ & $6.77 \mathrm{E}+01$ & $2.37 E+02$ & $0.00 \mathrm{E}+00$ & $3.16 \mathrm{E}+05$ & $1.26 \mathrm{E}+05$ & $5.99 \mathrm{E}+04$ & $1.20 \mathrm{E}+04$ \\
\hline S22 & $6.58 E+03$ & $0.00 E+00$ & $1.35 \mathrm{E}+03$ & $2.70 \mathrm{E}+02$ & $3.01 \mathrm{E}+01$ & $6.03 \mathrm{E}+00$ & $1.19 \mathrm{E}+01$ & $2.38 \mathrm{E}+00$ & $1.90 \mathrm{E}+04$ & $4.00 \mathrm{E}+04$ & $3.45 \mathrm{E}+02$ & $6.90 \mathrm{E}+01$ & $4.64 \mathrm{E}+06$ & $9.28 \mathrm{E}+05$ & $2.82 \mathrm{E}+05$ & $5.64 \mathrm{E}+04$ \\
\hline \multicolumn{17}{|c|}{ Values listed in Black Bold Italics print are detection limits or upper limits. } \\
\hline \multirow{2}{*}{\multicolumn{17}{|c|}{\begin{tabular}{l|l|l|l|} 
(1) The activities listed for Tank S4 are in units of $\mathrm{pCi} / \mathrm{L}$ and the mass is liters. & & \\
\end{tabular}}} \\
\hline \multirow{2}{*}{\multicolumn{15}{|c|}{$\begin{array}{l}\text { Tank S4 U-233 activity included in U-234 activity, Pu-240 activity included in Pu-239 activity. U-236, Pu-241, Pu-242, Cm-245, and Cm-246 not measured } \\
\text { and are based upon the calculated average activity concentrations of these nuclides in the other analyzed sludges. }\end{array}$}} & & \\
\hline \multicolumn{14}{|c|}{\begin{tabular}{|l|l|l|l|l} 
and are based upon the calculated average activity concentrations of these nuclides in the other analyzed sludges. & & \\
Tank S22 U-238 not measured. Calculated activity based upon average activity concentrations of the other analyzed sludges. &
\end{tabular}} & & & \\
\hline \multirow{2}{*}{\multicolumn{17}{|c|}{$\begin{array}{l}\text { Tank S22 U-238 not measured. Calculated activity based upon average activity concentrations of the other analyzed sludges. } \\
\text { Values listed in Blue Bold Italics are calculated based upon average activity concentrations of the analyzed sludge samples. }\end{array}$}} \\
\hline & & & & & & & & & & & & & & & & \\
\hline \multicolumn{17}{|c|}{ The sludge found in Tank S4 was not used to calculate average activity concentrations because of the fact that it was a liquid. } \\
\hline \multicolumn{17}{|c|}{\begin{tabular}{|l|l|l|} 
The average $\mathrm{U}-233$ activity concentration is based upon the sludge in Tank S19 only. & &
\end{tabular}} \\
\hline \multicolumn{17}{|c|}{ The calculated Pu-240 activity concentration is based upon the sludge in Tank S22 only. } \\
\hline \multicolumn{17}{|c|}{ The average Pu-242, Cm-245, and $\mathrm{Cm}-246$ activity concentrations were calculated from detection limit numbers. } \\
\hline Errors as & ssociated wi & th average $a$ & ctivities were & e calculated & by averagin & g nte rela & Cillos aso & & & & & & & & & \\
\hline
\end{tabular}




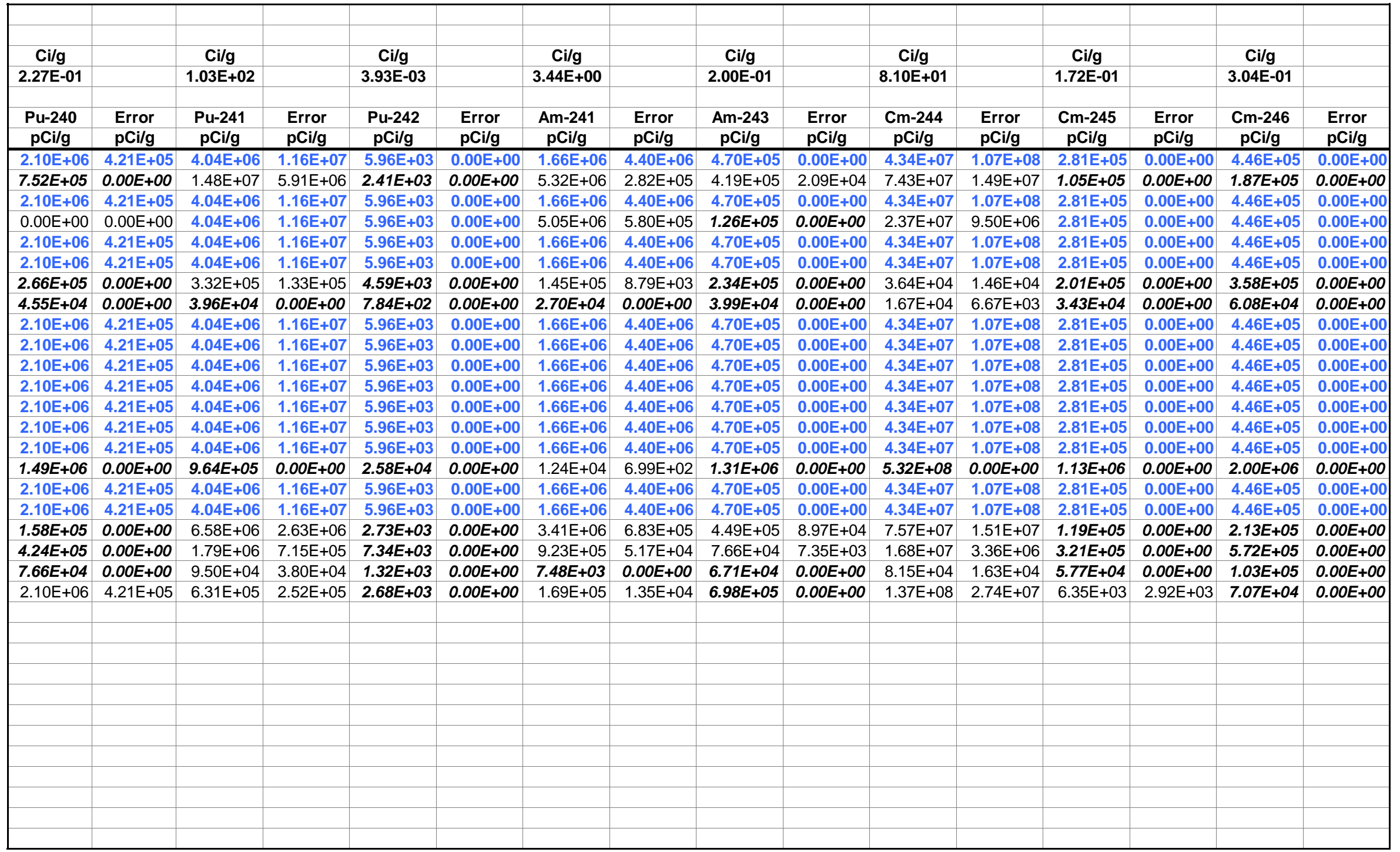




\begin{tabular}{|c|c|c|c|c|c|c|c|c|c|c|c|c|c|c|c|c|}
\hline \\
\hline \multicolumn{17}{|c|}{ Total Activity Including Error } \\
\hline & & & & & & & & & & & & & & & & \\
\hline & & & & & & & & & & & & & & & & \\
\hline Mass & U-233 & U-234 & U-235 & U-236 & U-238 & Np-237 & Pu-238 & Pu-239 & Pu-240 & Pu-241 & Pu-242 & Am-241 & Am-243 & $\mathrm{Cm}-244$ & Cm-245 & $\mathrm{Cm}-246$ \\
\hline Grams & $\mathbf{C i}$ & $\mathbf{C i}$ & $\mathbf{C i}$ & $\mathbf{C i}$ & $\mathrm{Ci}$ & $\mathrm{Ci}$ & $\mathrm{Ci}$ & $\mathrm{Ci}$ & $\mathrm{Ci}$ & $\mathrm{Ci}$ & $\mathrm{Ci}$ & $\mathrm{Ci}$ & $\mathbf{C i}$ & $\mathrm{Ci}$ & $\mathrm{Ci}$ & $\mathbf{C i}$ \\
\hline 34068.71 & 8.42E-03 & 6.58E-03 & 8.79E-05 & $1.15 \mathrm{E}-04$ & $2.01 \mathrm{E}-03$ & $2.33 E-04$ & $1.96 \mathrm{E}+00$ & 4.12E-02 & 8.59E-02 & 5.33E-01 & $2.03 E-04$ & $2.06 \mathrm{E}-01$ & 1.60E-02 & $5.12 \mathrm{E}+00$ & 9.57E-03 & $1.52 \mathrm{E}-02$ \\
\hline 251351.3 & $1.48 E-03$ & $2.54 E-03$ & $1.04 \mathrm{E}-05$ & $9.98 E-06$ & $2.49 \mathrm{E}-04$ & $2.69 E-04$ & $2.09 \mathrm{E}+01$ & 7.67E-02 & $1.89 E-01$ & $5.21 \mathrm{E}+00$ & $6.06 E-04$ & $1.41 \mathrm{E}+00$ & 1.11E-01 & $2.24 \mathrm{E}+01$ & $2.64 E-02$ & $4.70 E-02$ \\
\hline 28012.05 & 6.92E-03 & 5.41E-03 & 7.22E-05 & 9.47E-05 & 1.65E-03 & 1.92E-04 & $1.61 E+00$ & 3.39E-02 & 7.06E-02 & 4.38E-01 & 1.67E-04 & 1.70E-01 & 1.32E-02 & $4.21 E+00$ & 7.87E-03 & 1.25E-02 \\
\hline 21.95539 & $0.00 \mathrm{E}+00$ & $3.24 E-04$ & 7.84E-06 & $7.42 \mathrm{E}-08$ & $3.24 \mathrm{E}-04$ & 7.22E-06 & $2.56 \mathrm{E}-03$ & $1.39 E-04$ & $0.00 \mathrm{E}+00$ & $3.43 \mathrm{E}-04$ & 1.31E-07 & $1.24 \mathrm{E}-04$ & 2.77E-06 & 7.29E-04 & 6.17E-06 & 9.79E-06 \\
\hline 847932.2 & $2.10 \mathrm{E}-01$ & 1.64E-01 & $2.19 \mathrm{E}-03$ & $2.87 \mathrm{E}-03$ & $5.00 \mathrm{E}-02$ & $5.80 \mathrm{E}-03$ & $4.87 \mathrm{E}+01$ & $1.03 \mathrm{E}+00$ & $2.14 \mathrm{E}+00$ & $1.33 \mathrm{E}+01$ & $5.05 \mathrm{E}-03$ & $5.14 \mathrm{E}+00$ & 3.99E-01 & $1.28 \mathrm{E}+02$ & $2.38 \mathrm{E}-01$ & $3.78 \mathrm{E}-01$ \\
\hline 0 & $0.00 \mathrm{E}+00$ & $0.00 \mathrm{E}+00$ & $0.00 \mathrm{E}+00$ & $0.00 \mathrm{E}+00$ & $0.00 \mathrm{E}+00$ & $0.00 \mathrm{E}+00$ & $0.00 \mathrm{E}+00$ & $0.00 \mathrm{E}+00$ & $0.00 \mathrm{E}+00$ & $0.00 \mathrm{E}+00$ & $0.00 \mathrm{E}+00$ & $0.00 \mathrm{E}+00$ & $0.00 \mathrm{E}+00$ & $0.00 \mathrm{E}+00$ & $0.00 \mathrm{E}+00$ & $0.00 \mathrm{E}+00$ \\
\hline 11356.24 & $1.28 E-04$ & $2.34 \mathrm{E}-04$ & 7.92E-06 & $8.65 \mathrm{E}-06$ & $2.34 \mathrm{E}-04$ & 9.36E-06 & $3.21 \mathrm{E}-03$ & 5.52E-03 & $3.02 E-03$ & $5.28 \mathrm{E}-03$ & 5.21E-05 & $1.75 \mathrm{E}-03$ & $2.66 E-03$ & 5.79E-04 & $2.28 E-03$ & 4.07E-03 \\
\hline 45424.94 & $8.72 E-05$ & $5.63 E-05$ & $3.98 \mathrm{E}-07$ & $5.86 E-07$ & 9.05E-06 & 9.49E-06 & $1.73 E-02$ & $2.00 \mathrm{E}-03$ & $2.07 E-03$ & $1.80 E-03$ & $3.56 E-05$ & $1.23 E-03$ & $1.81 E-03$ & $1.06 \mathrm{E}-03$ & $1.56 E-03$ & $2.76 E-03$ \\
\hline 98420.71 & $2.43 \mathrm{E}-02$ & $1.90 \mathrm{E}-02$ & $2.54 \mathrm{E}-04$ & $3.33 \mathrm{E}-04$ & $5.81 \mathrm{E}-03$ & $6.73 \mathrm{E}-04$ & $5.65 \mathrm{E}+00$ & 1.19E-01 & $2.48 \mathrm{E}-01$ & $1.54 \mathrm{E}+00$ & $5.87 \mathrm{E}-04$ & $5.96 \mathrm{E}-01$ & $4.63 \mathrm{E}-02$ & $1.48 \mathrm{E}+01$ & $2.77 \mathrm{E}-02$ & 4.39E-02 \\
\hline 234695.5 & $5.80 \mathrm{E}-02$ & 4.53E-02 & $6.05 \mathrm{E}-04$ & 7.93E-04 & $1.38 \mathrm{E}-02$ & 1.61E-03 & $1.35 \mathrm{E}+01$ & 2.84E-01 & $5.92 \mathrm{E}-01$ & $3.67 \mathrm{E}+00$ & $1.40 \mathrm{E}-03$ & $1.42 \mathrm{E}+00$ & $1.10 \mathrm{E}-01$ & $3.53 \mathrm{E}+01$ & $6.59 \mathrm{E}-02$ & $1.05 \mathrm{E}-01$ \\
\hline 96149.46 & $2.38 \mathrm{E}-02$ & $1.86 \mathrm{E}-02$ & $2.48 \mathrm{E}-04$ & $3.25 \mathrm{E}-04$ & $5.67 \mathrm{E}-03$ & $6.58 \mathrm{E}-04$ & $5.52 \mathrm{E}+00$ & 1.16E-01 & 2.42E-01 & $1.50 \mathrm{E}+00$ & $5.73 \mathrm{E}-04$ & 5.83E-01 & 4.52E-02 & $1.45 \mathrm{E}+01$ & $2.70 \mathrm{E}-02$ & 4.29E-02 \\
\hline 37854.12 & $9.36 \mathrm{E}-03$ & 7.31E-03 & $9.76 \mathrm{E}-05$ & $1.28 \mathrm{E}-04$ & $2.23 \mathrm{E}-03$ & $2.59 \mathrm{E}-04$ & $2.17 \mathrm{E}+00$ & $4.58 \mathrm{E}-02$ & $9.54 \mathrm{E}-02$ & $5.92 \mathrm{E}-01$ & $2.26 \mathrm{E}-04$ & 2.29E-01 & $1.78 \mathrm{E}-02$ & $5.69 \mathrm{E}+00$ & $1.06 \mathrm{E}-02$ & $1.69 \mathrm{E}-02$ \\
\hline 325545.4 & $8.05 \mathrm{E}-02$ & $6.29 \mathrm{E}-02$ & $8.40 \mathrm{E}-04$ & $1.10 \mathrm{E}-03$ & $1.92 \mathrm{E}-02$ & $2.23 \mathrm{E}-03$ & $1.87 \mathrm{E}+01$ & 3.94E-01 & $8.21 \mathrm{E}-01$ & $5.09 \mathrm{E}+00$ & $1.94 \mathrm{E}-03$ & $1.97 \mathrm{E}+00$ & $1.53 \mathrm{E}-01$ & $4.90 \mathrm{E}+01$ & $9.15 \mathrm{E}-02$ & $1.45 \mathrm{E}-01$ \\
\hline 896385.5 & 2.22E-01 & $1.73 \mathrm{E}-01$ & $2.31 \mathrm{E}-03$ & $3.03 \mathrm{E}-03$ & $5.29 \mathrm{E}-02$ & 6.13E-03 & $5.15 \mathrm{E}+01$ & $1.08 \mathrm{E}+00$ & $2.26 \mathrm{E}+00$ & $1.40 \mathrm{E}+01$ & $5.34 \mathrm{E}-03$ & $5.43 \mathrm{E}+00$ & 4.21E-01 & $1.35 \mathrm{E}+02$ & $2.52 \mathrm{E}-01$ & $4.00 \mathrm{E}-01$ \\
\hline 1098527 & 2.72E-01 & 2.12E-01 & $2.83 \mathrm{E}-03$ & $3.71 \mathrm{E}-03$ & $6.48 \mathrm{E}-02$ & 7.51E-03 & $6.31 \mathrm{E}+01$ & $1.33 \mathrm{E}+00$ & $2.77 \mathrm{E}+00$ & $1.72 \mathrm{E}+01$ & $6.55 \mathrm{E}-03$ & $6.66 \mathrm{E}+00$ & $5.16 \mathrm{E}-01$ & $1.65 \mathrm{E}+02$ & $3.09 \mathrm{E}-01$ & $4.90 \mathrm{E}-01$ \\
\hline 2066835 & $1.30 \mathrm{E}-01$ & 8.43E-02 & $2.20 \mathrm{E}-03$ & $2.29 \mathrm{E}-03$ & $6.30 \mathrm{E}-02$ & $6.51 E-03$ & $2.14 \mathrm{E}+00$ & $1.78 \mathrm{E}+00$ & $3.08 E+00$ & $1.99 E+00$ & 5.33E-02 & 2.71E-02 & $2.71 E+00$ & $1.10 E+03$ & $2.34 E+00$ & $4.13 E+00$ \\
\hline 52995.77 & $1.31 \mathrm{E}-02$ & $1.02 \mathrm{E}-02$ & 1.37E-04 & $1.79 E-04$ & 3.13E-03 & $3.62 \mathrm{E}-04$ & $3.04 E+00$ & 6.41E-02 & 1.34E-01 & 8.29E-01 & 3.16E-04 & $3.21 \mathrm{E}-01$ & 2.49E-02 & $7.97 \mathrm{E}+00$ & 1.49E-02 & $2.36 \mathrm{E}-02$ \\
\hline 15141.65 & $3.74 \mathrm{E}-03$ & 2.93E-03 & 3.91E-05 & 5.12E-05 & 8.93E-04 & $1.04 \mathrm{E}-04$ & 8.69E-01 & 1.83E-02 & $3.82 E-02$ & 2.37E-01 & 9.02E-05 & 9.18E-02 & 7.12E-03 & $2.28 E+00$ & $4.25 \mathrm{E}-03$ & $6.75 \mathrm{E}-03$ \\
\hline 203655.2 & $5.03 \mathrm{E}-02$ & $3.22 \mathrm{E}-02$ & 7.09E-04 & 7.43E-04 & $1.01 \mathrm{E}-02$ & 1.17E-03 & $6.84 \mathrm{E}+00$ & $2.37 \mathrm{E}-01$ & $3.22 E-02$ & $1.88 \mathrm{E}+00$ & 5.56E-04 & 8.34E-01 & 1.10E-01 & $1.85 \mathrm{E}+01$ & $2.42 E-02$ & $4.34 E-02$ \\
\hline 68137.41 & $1.23 E-03$ & $7.90 E-04$ & $4.09 \mathrm{E}-05$ & $6.41 \mathrm{E}-05$ & $3.90 \mathrm{E}-03$ & $1.65 \mathrm{E}-04$ & 5.51E-01 & 6.66E-02 & $2.89 E-02$ & $1.71 \mathrm{E}-01$ & $5.00 E-04$ & 6.64E-02 & $5.72 \mathrm{E}-03$ & $1.37 \mathrm{E}+00$ & $2.19 E-02$ & $3.90 E-02$ \\
\hline 236966.8 & $7.70 E-04$ & $4.95 E-04$ & $4.09 \mathrm{E}-06$ & 5.17E-06 & $9.61 \mathrm{E}-05$ & 5.62E-05 & $1.05 \mathrm{E}-01$ & $1.70 \mathrm{E}-02$ & $1.82 E-02$ & $3.15 \mathrm{E}-02$ & $3.13 E-04$ & $1.77 E-03$ & $1.59 E-02$ & 2.32E-02 & $1.37 E-02$ & $2.44 E-02$ \\
\hline 56781.18 & $3.74 E-04$ & $9.20 \mathrm{E}-05$ & 2.05E-06 & $8.11 \mathrm{E}-07$ & 3.35E-03 & $2.35 \mathrm{E}-05$ & 3.16E-01 & $1.92 \mathrm{E}-02$ & $1.43 \mathrm{E}-01$ & $5.01 \mathrm{E}-02$ & $1.52 E-04$ & $1.04 \mathrm{E}-02$ & $3.96 E-02$ & $9.33 \mathrm{E}+00$ & $5.26 \mathrm{E}-04$ & $4.01 E-03$ \\
\hline & & & & & & & & & & & & & & & & \\
\hline & & & & & & & & & & & & & & & & \\
\hline & & & & & & & & & & & & & & & & \\
\hline & & & & & & & & & & & & & & & & \\
\hline & & & & & & & & & & & & & & & & \\
\hline & & & & & & & & & & & & & & & & \\
\hline & & & & & & & & & & & & & & & & \\
\hline & & & & & & & & & & & & & & & & \\
\hline & & & & & & & & & & & & & & & & \\
\hline & & & & & & & & & & & & & & & & \\
\hline & & & & & & & & & & & & & & & & \\
\hline & & & & & & & & & & & & & & & & \\
\hline
\end{tabular}




\begin{tabular}{|c|c|c|c|c|c|c|c|c|c|c|c|c|c|c|c|c|}
\hline \\
\hline \multicolumn{17}{|c|}{ Activity Concentration Including Error } \\
\hline & & & & & & & & & & & & & & & & \\
\hline & & & & & & & & & & & & & & & & \\
\hline & U-233 & U-234 & U-235 & U-236 & U-238 & Np-237 & Pu-238 & Pu-239 & Pu-240 & Pu-241 & Pu-242 & Am-241 & Am-243 & $\mathrm{Cm}-244$ & $\mathrm{Cm}-245$ & $\mathrm{Cm}-246$ \\
\hline Tank \# & $\mathrm{Ci} / \mathrm{g}$ & $\mathrm{Ci} / \mathrm{g}$ & $\mathrm{Ci} / \mathrm{g}$ & $\mathrm{Ci} / \mathrm{g}$ & $\mathrm{Ci} / \mathrm{g}$ & $\mathrm{Ci} / \mathrm{g}$ & $\mathrm{Ci} / \mathrm{g}$ & $\mathrm{Ci} / \mathrm{g}$ & $\mathrm{Ci} / \mathrm{g}$ & $\mathrm{Ci} / \mathrm{g}$ & $\mathrm{Ci} / \mathrm{g}$ & $\mathrm{Ci} / \mathrm{g}$ & $\mathrm{Ci} / \mathrm{g}$ & $\mathrm{Ci} / \mathrm{g}$ & $\mathrm{Ci} / \mathrm{g}$ & $\mathrm{Ci} / \mathrm{g}$ \\
\hline S1 & $2.47 E-07$ & 1.93E-07 & $2.58 E-09$ & $3.38 \mathrm{E}-09$ & 5.90E-08 & 6.84E-09 & 5.74E-05 & $1.21 \mathrm{E}-06$ & $2.52 E-06$ & 1.56E-05 & 5.96E-09 & $6.06 \mathrm{E}-06$ & 4.70E-07 & 1.50E-04 & $2.81 \mathrm{E}-07$ & 4.46E-07 \\
\hline S2 & 5.90E-09 & $1.01 E-08$ & $4.15 \mathrm{E}-11$ & 3.97E-11 & $9.89 E-10$ & $1.07 E-09$ & 8.33E-05 & $3.05 E-07$ & 7.52E-07 & 2.07E-05 & 2.41E-09 & 5.60E-06 & 4.40E-07 & 8.92E-05 & $1.05 E-07$ & 1.87E-07 \\
\hline S3 & 2.47E-07 & 1.93E-07 & 2.58E-09 & 3.38E-09 & 5.90E-08 & 6.84E-09 & 5.74E-05 & 1.21E-06 & 2.52E-06 & 1.56E-05 & 5.96E-09 & 6.06E-06 & 4.70E-07 & 1.50E-04 & 2.81E-07 & 4.46E-07 \\
\hline S4(1) & $0.00 \mathrm{E}+00$ & $1.48 E-05$ & 3.57E-07 & 3.38E-09 & 1.48E-05 & 3.29E-07 & 1.17E-04 & $6.34 E-06$ & $0.00 \mathrm{E}+00$ & 1.56E-05 & 5.96E-09 & 5.63E-06 & $1.26 E-07$ & 3.32E-05 & 2.81E-07 & 4.46E-07 \\
\hline S5 & 2.47E-07 & 1.93E-07 & 2.58E-09 & 3.38E-09 & 5.90E-08 & 6.84E-09 & 5.74E-05 & 1.21E-06 & 2.52E-06 & 1.56E-05 & 5.96E-09 & 6.06E-06 & 4.70E-07 & 1.50E-04 & 2.81E-07 & 4.46E-07 \\
\hline S6 & $0.00 \mathrm{E}+00$ & $0.00 E+00$ & $0.00 E+00$ & $0.00 E+00$ & $0.00 E+00$ & $0.00 E+00$ & $0.00 E+00$ & $0.00 E+00$ & $0.00 E+00$ & $0.00 \mathrm{E}+00$ & $0.00 E+00$ & $0.00 \mathrm{E}+00$ & $0.00 \mathrm{E}+00$ & $0.00 \mathrm{E}+00$ & $0.00 \mathrm{E}+00$ & $0.00 E+00$ \\
\hline S7 & $1.13 E-08$ & 2.06E-08 & $6.97 \mathrm{E}-10$ & 7.62E-10 & 2.06E-08 & $8.24 E-10$ & 2.83E-07 & $4.86 \mathrm{E}-07$ & 2.66E-07 & 4.65E-07 & 4.59E-09 & 1.54E-07 & $2.34 E-07$ & 5.10E-08 & $2.01 E-07$ & 3.58E-07 \\
\hline S8 & $1.92 E-09$ & $1.24 E-09$ & 8.76E-12 & $1.29 E-11$ & 1.99E-10 & $2.09 E-10$ & 3.81E-07 & 4.39E-08 & $4.55 E-08$ & $3.96 E-08$ & $7.84 E-10$ & 2.70E-08 & $3.99 E-08$ & 2.34E-08 & $3.43 E-08$ & $6.08 E-08$ \\
\hline S9 & 2.47E-07 & 1.93E-07 & 2.58E-09 & 3.38E-09 & 5.90E-08 & 6.84E-09 & 5.74E-05 & 1.21E-06 & 2.52E-06 & 1.56E-05 & 5.96E-09 & 6.06E-06 & 4.70E-07 & 1.50E-04 & 2.81E-07 & 4.46E-07 \\
\hline $\mathrm{S} 10$ & 2.47E-07 & 1.93E-07 & 2.58E-09 & 3.38E-09 & 5.90E-08 & 6.84E-09 & 5.74E-05 & 1.21E-06 & 2.52E-06 & 1.56E-05 & 5.96E-09 & 6.06E-06 & 4.70E-07 & 1.50E-04 & 2.81E-07 & 4.46E-07 \\
\hline $\mathrm{S} 11$ & 2.47E-07 & 1.93E-07 & 2.58E-09 & 3.38E-09 & 5.90E-08 & 6.84E-09 & 5.74E-05 & 1.21E-06 & 2.52E-06 & 1.56E-05 & 5.96E-09 & 6.06E-06 & 4.70E-07 & 1.50E-04 & 2.81E-07 & 4.46E-07 \\
\hline $\mathrm{S} 12$ & 2.47E-07 & 1.93E-07 & 2.58E-09 & 3.38E-09 & 5.90E-08 & 6.84E-09 & 5.74E-05 & 1.21E-06 & 2.52E-06 & 1.56E-05 & 5.96E-09 & 6.06E-06 & 4.70E-07 & 1.50E-04 & 2.81E-07 & 4.46E-07 \\
\hline $\mathrm{S} 13$ & 2.47E-07 & 1.93E-07 & 2.58E-09 & 3.38E-09 & 5.90E-08 & 6.84E-09 & 5.74E-05 & 1.21E-06 & 2.52E-06 & 1.56E-05 & 5.96E-09 & 6.06E-06 & 4.70E-07 & 1.50E-04 & 2.81E-07 & 4.46E-07 \\
\hline S14 & 2.47E-07 & 1.93E-07 & 2.58E-09 & 3.38E-09 & 5.90E-08 & 6.84E-09 & 5.74E-05 & 1.21E-06 & 2.52E-06 & 1.56E-05 & 5.96E-09 & 6.06E-06 & 4.70E-07 & 1.50E-04 & 2.81E-07 & 4.46E-07 \\
\hline $\mathrm{S} 15$ & 2.47E-07 & 1.93E-07 & 2.58E-09 & 3.38E-09 & 5.90E-08 & 6.84E-09 & 5.74E-05 & 1.21E-06 & 2.52E-06 & 1.56E-05 & 5.96E-09 & 6.06E-06 & 4.70E-07 & 1.50E-04 & 2.81E-07 & 4.46E-07 \\
\hline S16 & $6.31 E-08$ & $4.08 E-08$ & 1.06E-09 & 1.11E-09 & 3.05E-08 & 3.15E-09 & 1.03E-06 & 8.59E-07 & 1.49E-06 & 9.64E-07 & $2.58 E-08$ & 1.31E-08 & $1.31 E-06$ & 5.32E-04 & $1.13 E-06$ & $2.00 E-06$ \\
\hline S17 & 2.47E-07 & 1.93E-07 & 2.58E-09 & 3.38E-09 & 5.90E-08 & 6.84E-09 & 5.74E-05 & 1.21E-06 & 2.52E-06 & 1.56E-05 & 5.96E-09 & 6.06E-06 & 4.70E-07 & 1.50E-04 & 2.81E-07 & 4.46E-07 \\
\hline S18 & 2.47E-07 & 1.93E-07 & 2.58E-09 & 3.38E-09 & 5.90E-08 & 6.84E-09 & 5.74E-05 & 1.21E-06 & 2.52E-06 & 1.56E-05 & 5.96E-09 & 6.06E-06 & 4.70E-07 & 1.50E-04 & 2.81E-07 & 4.46E-07 \\
\hline S19 & $2.47 \mathrm{E}-07$ & $1.58 \mathrm{E}-07$ & $3.48 \mathrm{E}-09$ & $3.65 \mathrm{E}-09$ & 4.96E-08 & 5.73E-09 & $3.36 \mathrm{E}-05$ & $1.16 \mathrm{E}-06$ & $1.58 E-07$ & $9.21 \mathrm{E}-06$ & 2.73E-09 & 4.09E-06 & $5.39 \mathrm{E}-07$ & $9.08 \mathrm{E}-05$ & $1.19 E-07$ & 2.13E-07 \\
\hline S20 & $1.80 E-08$ & $1.16 E-08$ & $6.00 \mathrm{E}-10$ & $9.41 \mathrm{E}-10$ & 5.73E-08 & $2.42 \mathrm{E}-09$ & 8.08E-06 & $9.78 \mathrm{E}-07$ & 4.24E-07 & $2.51 \mathrm{E}-06$ & 7.34E-09 & $9.75 \mathrm{E}-07$ & $8.40 \mathrm{E}-08$ & 2.02E-05 & $3.21 E-07$ & $5.72 E-07$ \\
\hline S21 & $3.25 E-09$ & 2.09E-09 & $1.72 \mathrm{E}-11$ & $2.18 E-11$ & $4.06 \mathrm{E}-10$ & $2.37 E-10$ & 4.42E-07 & 7.19E-08 & $7.66 E-08$ & 1.33E-07 & 1.32E-09 & 7.48E-09 & $6.71 E-08$ & $9.78 \mathrm{E}-08$ & $5.77 E-08$ & $1.03 E-07$ \\
\hline $\mathrm{S} 22$ & $6.58 E-09$ & 1.62E-09 & $3.61 \mathrm{E}-11$ & $1.43 \mathrm{E}-11$ & 5.90E-08 & $4.14 \mathrm{E}-10$ & 5.57E-06 & 3.38E-07 & 2.52E-06 & 8.83E-07 & 2.68E-09 & 1.83E-07 & $6.98 E-07$ & 1.64E-04 & 9.27E-09 & 7.07E-08 \\
\hline & & & & & & & & & & & & & & & & \\
\hline & & & & & & & & & & & & & & & & \\
\hline & & & & & & & & & & & & & & & & \\
\hline & & & & & & & & & & & & & & & & \\
\hline & & & & & & & & & & & & & & & & \\
\hline & & & & & & & & & & & & & & & & \\
\hline & & & & & & & & & & & & & & & & \\
\hline & & & & & & & & & & & & & & & & \\
\hline & & & & & & & & & & & & & & & & \\
\hline & & & & & & & & & & & & & & & & \\
\hline & & & & & & & & & & & & & & & & \\
\hline & & & & & & & & & & & & & & & & \\
\hline
\end{tabular}




\begin{tabular}{|c|c|c|c|c|c|c|c|c|c|c|c|c|c|c|c|c|}
\hline & & & & & & & & & & & & & & & & \\
\hline & & & & & & & & & & & & & & & & \\
\hline & & & & & & & & & & & & & & & & \\
\hline & & & & & & & & & & & & & & & & \\
\hline & U-233 & U-234 & U-235 & U-236 & U-238 & Np-237 & Pu-238 & Pu-239 & Pu-240 & Pu-241 & Pu-242 & Am-241 & Am-243 & $\mathrm{Cm}-244$ & $\mathrm{Cm}-245$ & $\mathrm{Cm}-246$ \\
\hline Tank \# & g & g & $\mathrm{g}$ & g & g & $\mathrm{g}$ & g & g & g & g & g & g & $\mathrm{g}$ & g & $\mathrm{g}$ & g \\
\hline S1 & 8.74E-01 & $1.05 E+00$ & $4.07 \mathrm{E}+01$ & $1.78 \mathrm{E}+00$ & $5.98 \mathrm{E}+03$ & 3.31E-01 & 1.14E-01 & 6.63E-01 & $3.78 \mathrm{E}-01$ & $5.15 \mathrm{E}-03$ & $5.17 \mathrm{E}-02$ & $6.00 \mathrm{E}-02$ & $8.01 \mathrm{E}-02$ & $6.33 \mathrm{E}-02$ & $5.57 \mathrm{E}-02$ & $5.00 \mathrm{E}-02$ \\
\hline S2 & $1.54 E-01$ & 4.07E-01 & $4.83 \mathrm{E}+00$ & $1.54 E-01$ & $7.40 \mathrm{E}+02$ & $3.81 E-01$ & $1.22 \mathrm{E}+00$ & $1.23 E+00$ & $8.33 E-01$ & 5.03E-02 & $1.54 E-01$ & 4.09E-01 & 5.53E-01 & 2.77E-01 & $1.53 E-01$ & $1.55 E-01$ \\
\hline S3 & 7.18E-01 & 8.67E-01 & $3.34 E+01$ & $1.46 E+00$ & $4.92 E+03$ & $2.72 E-01$ & $9.40 \mathrm{E}-02$ & 5.45E-01 & 3.11E-01 & 4.24E-03 & 4.25E-02 & 4.93E-02 & 6.58E-02 & $5.20 \mathrm{E}-02$ & 4.58E-02 & 4.11E-02 \\
\hline S4(1) & $0.00 \mathrm{E}+00$ & 5.20E-02 & $3.63 E+00$ & 1.15E-03 & $9.65 E+02$ & $1.02 E-02$ & $1.50 \mathrm{E}-04$ & $2.24 E-03$ & $0.00 \mathrm{E}+00$ & 3.32E-06 & 3.33E-05 & 3.59E-05 & $1.38 E-05$ & 9.00E-06 & 3.59E-05 & 3.22E-05 \\
\hline S5 & $2.17 \mathrm{E}+01$ & $2.63 E+01$ & $1.01 \mathrm{E}+03$ & 4.43E+01 & $1.49 \mathrm{E}+05$ & $8.23 E+00$ & $2.85 \mathrm{E}+00$ & $1.65 \mathrm{E}+01$ & $9.42 \mathrm{E}+00$ & 1.28E-01 & $1.29 E+00$ & $1.49 E+00$ & $1.99 \mathrm{E}+00$ & $1.57 \mathrm{E}+00$ & $1.39 E+00$ & $1.24 E+00$ \\
\hline S6 & $0.00 E+00$ & $0.00 \mathrm{E}+00$ & $0.00 \mathrm{E}+00$ & $0.00 \mathrm{E}+00$ & $0.00 E+00$ & $0.00 \mathrm{E}+00$ & $0.00 \mathrm{E}+00$ & $0.00 \mathrm{E}+00$ & $0.00 E+00$ & $0.00 E+00$ & $0.00 \mathrm{E}+00$ & $0.00 E+00$ & $0.00 E+00$ & $0.00 E+00$ & $0.00 E+00$ & $0.00 E+00$ \\
\hline S7 & 1.33E-02 & 3.75E-02 & $3.66 \mathrm{E}+00$ & 1.34E-01 & $6.98 \mathrm{E}+02$ & $1.33 E-02$ & 1.88E-04 & 8.89E-02 & $1.33 E-02$ & 5.11E-05 & $1.33 E-02$ & 5.08E-04 & 1.33E-02 & 7.15E-06 & 1.33E-02 & $1.34 E-02$ \\
\hline S8 & $9.05 E-03$ & 9.03E-03 & 1.84E-01 & $9.06 E-03$ & $2.69 \mathrm{E}+01$ & $1.35 E-02$ & $1.01 E-03$ & $3.21 \mathrm{E}-02$ & $9.10 E-03$ & $1.74 E-05$ & 9.06E-03 & $3.57 E-04$ & 9.06E-03 & 1.31E-05 & 9.06E-03 & 9.08E-03 \\
\hline S9 & $2.52 E+00$ & $3.05 E+00$ & $1.18 \mathrm{E}+02$ & $5.14 \mathrm{E}+00$ & $1.73 E+04$ & 9.55E-01 & 3.30E-01 & $1.92 \mathrm{E}+00$ & $1.09 \mathrm{E}+00$ & 1.49E-02 & 1.49E-01 & 1.73E-01 & 2.31E-01 & 1.83E-01 & 1.61E-01 & 1.44E-01 \\
\hline $\mathrm{S} 10$ & $6.02 E+00$ & $7.27 \mathrm{E}+00$ & $2.80 \mathrm{E}+02$ & $1.23 \mathrm{E}+01$ & $4.12 \mathrm{E}+04$ & $2.28 \mathrm{E}+00$ & 7.88E-01 & 4.57E+00 & $2.61 E+00$ & $3.55 E-02$ & 3.56E-01 & 4.13E-01 & 5.52E-01 & 4.36E-01 & 3.83E-01 & 3.44E-01 \\
\hline $\mathrm{S} 11$ & $2.47 E+00$ & $2.98 \mathrm{E}+00$ & $1.15 \mathrm{E}+02$ & $5.02 \mathrm{E}+00$ & $1.69 E+04$ & 9.33E-01 & 3.23E-01 & $1.87 \mathrm{E}+00$ & $1.07 E+00$ & 1.45E-02 & 1.46E-01 & 1.69E-01 & 2.26E-01 & 1.79E-01 & 1.57E-01 & 1.41E-01 \\
\hline $\mathrm{S} 12$ & 9.71E-01 & $1.17 \mathrm{E}+00$ & $4.52 \mathrm{E}+01$ & $1.98 \mathrm{E}+00$ & $6.65 E+03$ & 3.67E-01 & 1.27E-01 & 7.37E-01 & $4.20 \mathrm{E}-01$ & 5.73E-03 & 5.74E-02 & 6.67E-02 & 8.90E-02 & 7.03E-02 & 6.18E-02 & 5.55E-02 \\
\hline $\mathrm{S} 13$ & $8.35 E+00$ & $1.01 \mathrm{E}+01$ & $3.89 \mathrm{E}+02$ & $1.70 \mathrm{E}+01$ & $5.72 \mathrm{E}+04$ & $3.16 \mathrm{E}+00$ & $1.09 \mathrm{E}+00$ & $6.34 \mathrm{E}+00$ & $3.62 \mathrm{E}+00$ & 4.92E-02 & 4.94E-01 & 5.73E-01 & 7.65E-01 & 6.04E-01 & 5.32E-01 & 4.78E-01 \\
\hline S14 & $2.30 \mathrm{E}+01$ & $2.78 \mathrm{E}+01$ & 1.07E+03 & $4.68 \mathrm{E}+01$ & $1.57 \mathrm{E}+05$ & $8.70 \mathrm{E}+00$ & $3.01 E+00$ & $1.75 \mathrm{E}+01$ & $9.96 \mathrm{E}+00$ & 1.36E-01 & $1.36 \mathrm{E}+00$ & $1.58 \mathrm{E}+00$ & $2.11 E+00$ & $1.66 \mathrm{E}+00$ & $1.46 \mathrm{E}+00$ & $1.32 E+00$ \\
\hline $\mathrm{S} 15$ & $2.82 E+01$ & $3.40 \mathrm{E}+01$ & $1.31 \mathrm{E}+03$ & $5.74 \mathrm{E}+01$ & $1.93 E+05$ & 1.07E+01 & $3.69 \mathrm{E}+00$ & $2.14 \mathrm{E}+01$ & $1.22 \mathrm{E}+01$ & 1.66E-01 & $1.67 \mathrm{E}+00$ & $1.94 \mathrm{E}+00$ & $2.58 E+00$ & $2.04 E+00$ & $1.79 E+00$ & $1.61 E+00$ \\
\hline S16 & $1.35 E+01$ & $1.35 E+01$ & $1.02 \mathrm{E}+03$ & $3.54 \mathrm{E}+01$ & $1.87 \mathrm{E}+05$ & $9.23 E+00$ & $1.25 \mathrm{E}-01$ & $2.86 \mathrm{E}+01$ & $1.36 E+01$ & 1.93E-02 & $1.36 E+01$ & 7.87E-03 & $1.35 E+01$ & $1.36 E+01$ & $1.36 E+01$ & $1.36 E+01$ \\
\hline $\mathrm{S} 17$ & $1.36 \mathrm{E}+00$ & $1.64 \mathrm{E}+00$ & $6.33 E+01$ & $2.77 \mathrm{E}+00$ & $9.31 E+03$ & 5.14E-01 & 1.78E-01 & $1.03 E+00$ & 5.89E-01 & 8.02E-03 & 8.04E-02 & 9.34E-02 & 1.25E-01 & 9.84E-02 & 8.66E-02 & 7.78E-02 \\
\hline S18 & 3.88E-01 & 4.69E-01 & 1.81E+01 & 7.91E-01 & $2.66 \mathrm{E}+03$ & 1.47E-01 & 5.08E-02 & 2.95E-01 & 1.68E-01 & 2.29E-03 & 2.30E-02 & 2.67E-02 & 3.56E-02 & 2.81E-02 & 2.47E-02 & 2.22E-02 \\
\hline S19 & $5.22 \mathrm{E}+00$ & $5.17 \mathrm{E}+00$ & $3.28 \mathrm{E}+02$ & $1.15 \mathrm{E}+01$ & $3.00 \mathrm{E}+04$ & $1.65 \mathrm{E}+00$ & 4.00E-01 & $3.81 \mathrm{E}+00$ & $1.42 E-01$ & 1.81E-02 & $1.41 E-01$ & 2.42E-01 & 5.49E-01 & 2.28E-01 & $1.41 E-01$ & $1.43 E-01$ \\
\hline $\mathrm{S} 20$ & $1.27 E-01$ & $1.27 E-01$ & $1.89 \mathrm{E}+01$ & 9.91E-01 & $1.16 \mathrm{E}+04$ & 2.34E-01 & 3.22E-02 & 1.07E+00 & $1.27 E-01$ & 1.65E-03 & $1.27 E-01$ & 1.93E-02 & 2.86E-02 & 1.70E-02 & 1.27E-01 & $1.28 E-01$ \\
\hline $\mathrm{S} 21$ & 7.99E-02 & $7.94 E-02$ & $1.89 \mathrm{E}+00$ & 7.98E-02 & $2.86 \mathrm{E}+02$ & 7.97E-02 & $6.13 \mathrm{E}-03$ & $2.74 \mathrm{E}-01$ & $8.00 E-02$ & 3.05E-04 & 7.96E-02 & $5.15 E-04$ & 7.95E-02 & 2.86E-04 & 7.95E-02 & 8.03E-02 \\
\hline $\mathrm{S} 22$ & $3.88 E-02$ & 1.47E-02 & 9.50E-01 & $1.25 \mathrm{E}-02$ & $9.97 \mathrm{E}+03$ & 3.33E-02 & 1.85E-02 & 3.09E-01 & 6.31E-01 & 4.85E-04 & 3.87E-02 & 3.01E-03 & $1.98 E-01$ & 1.15E-01 & 3.06E-03 & $1.32 E-02$ \\
\hline & & & & & & & & & & & & & & & & \\
\hline & & & & & & & & & & & & & & & & \\
\hline & & & & & & & & & & & & & & & & \\
\hline & & & & & & & & & & & & & & & & \\
\hline & & & & & & & & & & & & & & & & \\
\hline & & & & & & & & & & & & & & & & \\
\hline & & & & & & & & & & & & & & & & \\
\hline & & & & & & & & & & & & & & & & \\
\hline & & & & & & & & & & & & & & & & \\
\hline & & & & & & & & & & & & & & & & \\
\hline
\end{tabular}




\begin{tabular}{|c|c|c|c|c|c|c|c|c|c|c|c|c|c|c|}
\hline & & & & & & & & & & & & & & \\
\hline & & & & & & & & & & & & & & \\
\hline & & & & & & & & & & & & & & \\
\hline & & & & & & & & & & & & & & \\
\hline & U-233 & U-234 & U-235 & U-236 & U-238 & Np-237 & Pu-238 & Pu-239 & Pu-240 & Pu-241 & Pu-242 & $A m-241$ & Am-243 & $\mathrm{Cm}-244$ \\
\hline Tank \# & $\mathrm{g} /$ (g waste) & g/(g waste) & $\mathrm{g} /$ (g waste) & g/(g waste) & g/(g waste) & g/(g waste) & g/(g waste) & g/(g waste) & g/(g waste) & g/(g waste) & g/(g waste) & $\mathrm{g} /$ (g waste) & $g /(g$ waste $)$ & g/(g waste) \\
\hline S1 & $2.56 \mathrm{E}-05$ & $3.10 \mathrm{E}-05$ & $1.19 \mathrm{E}-03$ & $5.22 \mathrm{E}-05$ & $1.76 \mathrm{E}-01$ & $9.70 \mathrm{E}-06$ & $3.36 \mathrm{E}-06$ & $1.95 \mathrm{E}-05$ & $1.11 \mathrm{E}-05$ & $1.51 \mathrm{E}-07$ & $1.52 \mathrm{E}-06$ & $1.76 \mathrm{E}-06$ & $2.35 \mathrm{E}-06$ & $1.86 \mathrm{E}-06$ \\
\hline S2 & $6.12 E-07$ & $1.62 E-06$ & 1.92E-05 & $6.14 E-07$ & 2.94E-03 & $1.52 E-06$ & 4.87E-06 & 4.91E-06 & $3.31 E-06$ & 2.00E-07 & $6.13 E-07$ & 1.63E-06 & $2.20 \mathrm{E}-06$ & 1.10E-06 \\
\hline S3 & 2.56E-05 & 3.10E-05 & 1.19E-03 & 5.22E-05 & 1.76E-01 & 9.70E-06 & 3.36E-06 & 1.95E-05 & 1.11E-05 & 1.51E-07 & 1.52E-06 & 1.76E-06 & 2.35E-06 & 1.86E-06 \\
\hline S4(1) & $0.00 \mathrm{E}+00$ & $2.37 E-03$ & $1.65 E-01$ & 5.22E-05 & $4.40 \mathrm{E}+01$ & $4.67 E-04$ & 6.83E-06 & $1.02 E-04$ & $0.00 \mathrm{E}+00$ & 1.51E-07 & 1.52E-06 & 1.64E-06 & 6.30E-07 & $4.10 \mathrm{E}-07$ \\
\hline S5 & 2.56E-05 & 3.10E-05 & 1.19E-03 & 5.22E-05 & 1.76E-01 & 9.70E-06 & 3.36E-06 & 1.95E-05 & 1.11E-05 & 1.51E-07 & 1.52E-06 & 1.76E-06 & 2.35E-06 & 1.86E-06 \\
\hline S6 & $0.00 E+00$ & $0.00 \mathrm{E}+00$ & $0.00 E+00$ & $0.00 E+00$ & $0.00 E+00$ & $0.00 \mathrm{E}+00$ & $0.00 \mathrm{E}+00$ & $0.00 E+00$ & $0.00 E+00$ & $0.00 E+00$ & $0.00 \mathrm{E}+00$ & $0.00 \mathrm{E}+00$ & $0.00 E+00$ & $0.00 E+00$ \\
\hline S7 & 1.17E-06 & 3.31E-06 & 3.23E-04 & 1.18E-05 & $6.14 \mathrm{E}-02$ & 1.17E-06 & $1.65 \mathrm{E}-08$ & 7.82E-06 & 1.17E-06 & $4.50 \mathrm{E}-09$ & 1.17E-06 & 4.47E-08 & 1.17E-06 & $6.30 \mathrm{E}-10$ \\
\hline S8 & 1.99E-07 & $1.99 E-07$ & $4.06 \mathrm{E}-06$ & $1.99 E-07$ & 5.93E-04 & $2.96 E-07$ & $2.23 E-08$ & 7.07E-07 & $2.00 E-07$ & $3.83 E-10$ & $1.99 E-07$ & $7.85 E-09$ & $2.00 E-07$ & $2.89 \mathrm{E}-10$ \\
\hline S9 & 2.56E-05 & $3.10 \mathrm{E}-05$ & 1.19E-03 & 5.22E-05 & 1.76E-01 & $9.70 \mathrm{E}-06$ & 3.36E-06 & 1.95E-05 & 1.11E-05 & 1.51E-07 & 1.52E-06 & 1.76E-06 & $2.35 \mathrm{E}-06$ & 1.86E-06 \\
\hline $\mathrm{S} 10$ & 2.56E-05 & 3.10E-05 & 1.19E-03 & 5.22E-05 & 1.76E-01 & $9.70 \mathrm{E}-06$ & 3.36E-06 & 1.95E-05 & 1.11E-05 & 1.51E-07 & $1.52 \mathrm{E}-06$ & 1.76E-06 & 2.35E-06 & 1.86E-06 \\
\hline S11 & 2.56E-05 & 3.10E-05 & 1.19E-03 & 5.22E-05 & 1.76E-01 & 9.70E-06 & 3.36E-06 & 1.95E-05 & 1.11E-05 & 1.51E-07 & 1.52E-06 & 1.76E-06 & 2.35E-06 & 1.86E-06 \\
\hline S12 & 2.56E-05 & 3.10E-05 & 1.19E-03 & 5.22E-05 & 1.76E-01 & 9.70E-06 & 3.36E-06 & 1.95E-05 & 1.11E-05 & 1.51E-07 & 1.52E-06 & 1.76E-06 & 2.35E-06 & 1.86E-06 \\
\hline $\mathrm{S} 13$ & 2.56E-05 & 3.10E-05 & 1.19E-03 & 5.22E-05 & 1.76E-01 & 9.70E-06 & 3.36E-06 & 1.95E-05 & 1.11E-05 & 1.51E-07 & 1.52E-06 & 1.76E-06 & 2.35E-06 & 1.86E-06 \\
\hline S14 & 2.56E-05 & 3.10E-05 & 1.19E-03 & 5.22E-05 & 1.76E-01 & 9.70E-06 & 3.36E-06 & 1.95E-05 & 1.11E-05 & 1.51E-07 & 1.52E-06 & 1.76E-06 & 2.35E-06 & 1.86E-06 \\
\hline $\mathrm{S} 15$ & $2.56 \mathrm{E}-05$ & 3.10E-05 & 1.19E-03 & 5.22E-05 & 1.76E-01 & 9.70E-06 & 3.36E-06 & 1.95E-05 & 1.11E-05 & 1.51E-07 & 1.52E-06 & 1.76E-06 & 2.35E-06 & 1.86E-06 \\
\hline S16 & $6.55 E-06$ & $6.54 E-06$ & 4.93E-04 & 1.71E-05 & 9.07E-02 & 4.47E-06 & 6.05E-08 & 1.38E-05 & $6.56 E-06$ & 9.32E-09 & $6.56 E-06$ & 3.81E-09 & $6.55 E-06$ & 6.57E-06 \\
\hline S17 & 2.56E-05 & 3.10E-05 & 1.19E-03 & 5.22E-05 & 1.76E-01 & 9.70E-06 & 3.36E-06 & 1.95E-05 & 1.11E-05 & 1.51E-07 & 1.52E-06 & 1.76E-06 & 2.35E-06 & 1.86E-06 \\
\hline S18 & 2.56E-05 & 3.10E-05 & 1.19E-03 & 5.22E-05 & 1.76E-01 & 9.70E-06 & 3.36E-06 & 1.95E-05 & 1.11E-05 & 1.51E-07 & 1.52E-06 & 1.76E-06 & 2.35E-06 & 1.86E-06 \\
\hline S19 & 2.56E-05 & 2.54E-05 & 1.61E-03 & 5.64E-05 & 1.47E-01 & $8.12 \mathrm{E}-06$ & 1.96E-06 & 1.87E-05 & $6.96 E-07$ & 8.91E-08 & $6.95 E-07$ & 1.19E-06 & 2.69E-06 & 1.12E-06 \\
\hline $\mathrm{S} 20$ & 1.87E-06 & $1.86 E-06$ & $2.78 \mathrm{E}-04$ & 1.45E-05 & 1.70E-01 & 3.44E-06 & 4.73E-07 & 1.57E-05 & $1.87 E-06$ & 2.42E-08 & 1.87E-06 & 2.83E-07 & $4.20 \mathrm{E}-07$ & $2.49 \mathrm{E}-07$ \\
\hline S21 & 3.37E-07 & $3.35 E-07$ & 7.98E-06 & 3.37E-07 & $1.21 \mathrm{E}-03$ & $3.36 E-07$ & $2.58 \mathrm{E}-08$ & 1.16E-06 & 3.37E-07 & 1.29E-09 & $3.36 E-07$ & $2.17 E-09$ & $3.36 E-07$ & 1.21E-09 \\
\hline S22 & $6.83 E-07$ & 2.60E-07 & 1.67E-05 & 2.21E-07 & 1.76E-01 & 5.87E-07 & $3.26 \mathrm{E}-07$ & $5.45 \mathrm{E}-06$ & 1.11E-05 & 8.54E-09 & $6.82 E-07$ & 5.31E-08 & $3.49 E-06$ & 2.03E-06 \\
\hline & & & & & & & & & & & & & & \\
\hline & & & & & & & & & & & & & & \\
\hline & & & & & & & & & & & & & & \\
\hline & & & & & & & & & & & & & & \\
\hline & & & & & & & & & & & & & & \\
\hline & & & & & & & & & & & & & & \\
\hline & & & & & & & & & & & & & & \\
\hline & & & & & & & & & & & & & & \\
\hline & & & & & & & & & & & & & & \\
\hline & & & & & & & & & & & & & & \\
\hline & & & & & & & & & & & & & & \\
\hline & & & & & & & & & & & & & & \\
\hline
\end{tabular}


Appendix 4

WSRC-TR-2000-00011

Revision 0

January 21, 2000

Page 47

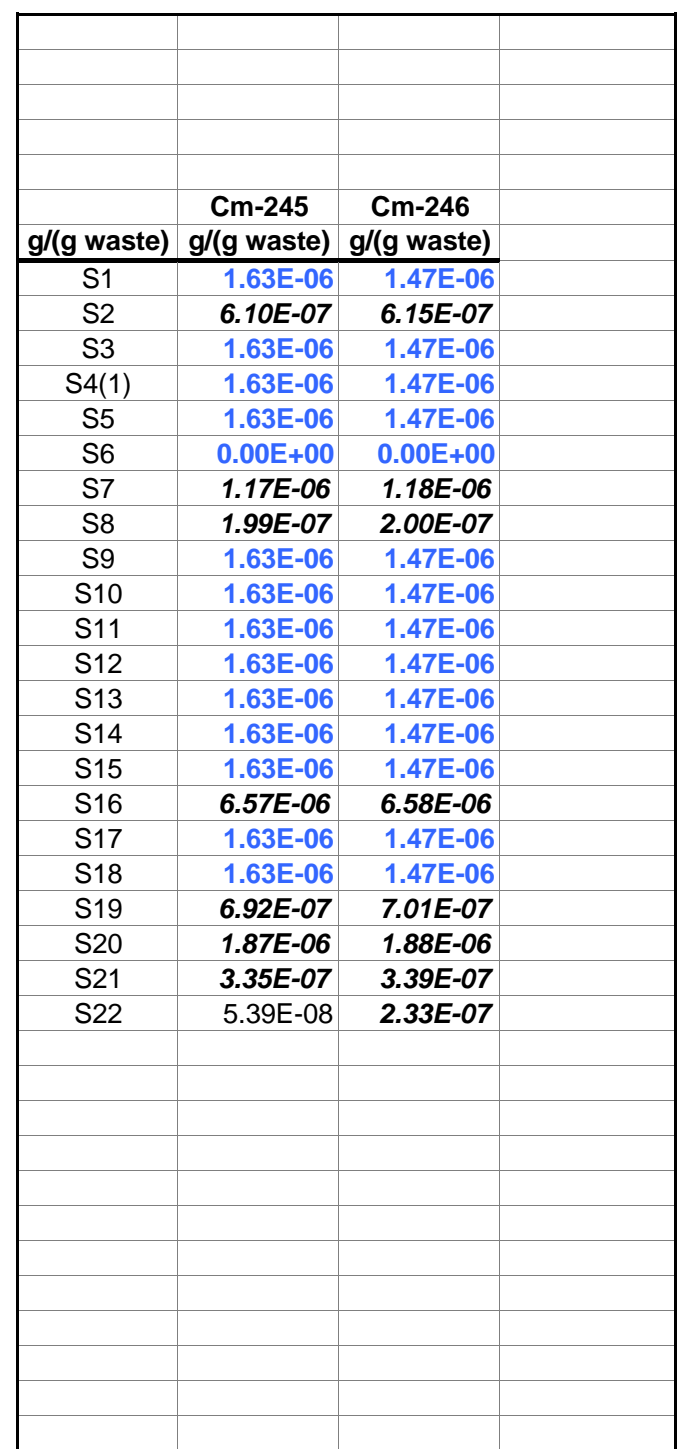

